\title{
O método do referencial móvel e sistemas diferenciais exteriores
}

Carlos Henrique Silva Alcântara

\author{
DiSSERTAÇÃO APRESENTADA
}

$\mathrm{AO}$

Instituto DE Matemática e Estatística

DA

Universidade De SÃo Paulo

PARA

OBTENÇÃO DO TÍTULO

$\mathrm{DE}$

Mestre em CiênCIAS

Programa: Matemática

Orientador: Prof. Dr. Cláudio Gorodski

Durante o desenvolvimento deste trabalho o autor recebeu auxílio financeiro FAPESP, processo: $2017 / 08206-8$

Sâo Paulo, julho de 2019 



\section{O método do referencial móvel e sistemas diferenciais exteriores}

Esta é a versão corrigida da dissertação elaborada pelo candidato Carlos Henrique Silva Alcântara, contendo as modificações sugeridas pela Comissão Julgadora. 



\section{Agradecimentos}

Gostaria de agradecer ao meu orientador Cláudio Gorodski pela atenção e paciência, ao Instituto de Matemática e Estatística como um todo por proporcionar o ambiente fértil para execução desse trabalho e a FAPESP pelo suporte financeiro.

Agradeço à minha família pelo suporte e carinho.

Agradeço aos meus amigos: Reinaldo, pela parceria em Geometria; Vinícius, pelo exemplo de dedicação; Marcos, por me ajudar a entender as principais ideias desse texto.

Agradeço à minha companheira Renata, pelo amor que já perdura por mais de uma década, pela inspiração que sua presença cativa em mim, pela profunda amizade que conquistamos, pelo total suporte nos momentos mais difíceis e a pela oportunidade de trilharmos nossos caminhos de mãos dadas. 


\section{Resumo}

Alcântara, C. H. S. O método do referencial móvel e sistemas diferenciais exteriores. 2019. Dissertação (Mestrado) - Instituto de Matemática e Estatística, Universidade de São Paulo, São Paulo, 2019.

Nesse trabalho, estudamos o método do referencial móvel e sistemas diferenciais exteriores. Estabelecemos resultados de Geometria Riemanniana via referenciais móveis e com essa linguagem introduzimos o Teorema de Gauss-Bonnet-Chern e apresentamos uma adaptação da demonstração original de S.-S. Chern presente no artigo A simple intrinsic proof of the Gauss-Bonnet formula for closed Riemannian manifolds. Ao abordar aspectos da teoria de Cartan-Kähler, codificamos as ideias oriundas dos referenciais móveis em sistemas diferenciais exteriores e mostramos algumas aplicações à Geometria Riemanniana.

Palavras-chave: referenciais móveis, fórmula de Gauss-Bonnet, teoria de Cartan-Kähler. 


\section{Abstract}

Alcântara, C. H. S. Moving frames and exterior differential systtems. 2019. Dissertação (Mestrado) - Instituto de Matemática e Estatística, Universidade de São Paulo, São Paulo, 2019.

In this work, we study the method of moving frame and exterior differential systems. We set up results of Riemannian Geometry via moving frames and with this language we introduce the Gauss-Bonnet-Chern Theorem and present an adaptation of the original proof of S.-S. Chern in the article A simple intrinsic proof of the Gauss-Bonnet formula for closed Riemannian manifolds. In discussing aspects of Cartan-Kähler's theory, we encode the ideas from moving frames into exterior differential systems and use this tool in Riemannian Geometry.

Keywords: moving frames, Gauss-Bonnet formula, Cartan-Kähler theory. 


\section{Sumário}

0 Introdução 3

1 Geometria Riemanniana via Referenciais Móveis $\quad 7$

1.1 Sistemas diferenciais exteriores . . . . . . . . . . . . . . . . . . . . 7

1.2 A forma de Maurer-Cartan . . . . . . . . . . . . . . . . . . . . . 9

1.3 Curvas e superfícies via referenciais móveis . . . . . . . . . . . . 15

1.4 Geometria intrínseca . . . . . . . . . . . . . . . . . 21

2 Fórmula de Gauss-Bonnet-Chern 33

2.1 Preliminares . . . . . . . . . . . . . . . . . . . . . . 33

2.2 Transgressão de $\Omega \ldots \ldots \ldots \ldots \ldots \ldots \ldots$

2.3 Demonstração da fórmula de Gauss-Bonnet-Chern . . . . . . . . . . . . . 42

$3 \quad$ Teoria de Cartan-Kähler $\quad 47$

3.1 Cartan-Kähler para tableaux . . . . . . . . . . . . . . . 47

3.2 Cartan-Kähler para sistemas Pfaffian lineares . . . . . . . . . . . . . 52

3.3 Cartan-Kähler geral . . . . . . . . . . . . . . . . . . . . 75

$\begin{array}{ll}\text { A Característica de Cauchy } & 95\end{array}$

A.1 Simetrias. . . . . . . . . . . . . . . . . . . . 95

A.2 Campos característicos . . . . . . . . . . . . . . . 97

$\begin{array}{ll}\text { Referências Bibliográficas } & 103\end{array}$ 


\section{Introdução}

Nesta dissertação estudamos aspectos da teoria do referencial móvel e também utilizamos essa linguagem para introduzir conceitos da teoria de Cartan-Kähler. Paralelamente apresentamos a demonstração da fórmula de Gauss-Bonnet-Chern devida a S.-S.Chern.

A teoria dos referenciais móveis para superfícies, segundo [M. A. Akivis, 1993], começa no final do século XIX com Gaston Darboux e Émile Cotton estudando o problema de construir um referencial móvel adaptado ao longo de superfícies no espaço Euclidiano ao invés de curvas, uma vez que este caso já estava amplamente discutido por Bertls, Serret, Frênet e outros. O método do referencial móvel em superfícies foi apresentado sistematicamente por Darboux em suas notas Leçons sur la théorie générale des surfaces [Darboux, 1887]. Para estudar curvas em superfícies, Darboux considerou um triedro formado por vetores $e_{1}, e_{2}$ e $e_{3}$, em que $e_{1}$ e $e_{3}$ eram paralelos à reta tangente à curva e a reta normal à superfície respectivamente, e para estudar superfícies considerou um novo triedro em que os vetores $e_{1}$ e $e_{2}$ eram paralelos às linhas de curvatura e $e_{3}$ normal à superfície. No segundo caso, Darboux considerou as derivadas do referencial segundo o comprimento de arco das linhas de curvatura, e os coeficientes dessas derivadas em relação à decomposição pelo triedro eram justamente as curvaturas principais da superfície, as curvaturas geodésicas e torção geodésica das linhas de curvatura. Em 1905, Émile Cotton publicou o artigo Generalisation de la theorie du triedre mobile [Émile Cotton, 1905], em que introduziu a generalização do método do referencial móvel para espaços $E$ que possuíam uma ação contínua e transitiva de um grupo $G$.

Apesar da vasta teoria desenvolvida no final do século XIX e começo do século XX, a ideia de referenciais móveis está muito atrelada ao nome de Élie Cartan, responsável por transformar a teoria em um poderoso algoritmo para estudar aspectos geométricos de subvariedades de espaços homogêneos e seus invariantes sob a ação de grupos de transformações. Desenvolvendo as ideias de Darboux e Cotton, em 1910 Cartan publicou os 
artigos Sur les développables isotropes et la méthode du trièdre mobile [Élie Cartan, 1955b] e La structure des groupes de transformations continus et la thèorie du trièdre mobile [Élie Cartan, 1955a]. Em especial, no segundo artigo Cartan conecta o método do referencial móvel via triedros com a estrutura de grupos de Lie e a teoria de equações de Pfaff, o que mais tarde ficou conhecido, de fato, como método do referencial móvel.

Cartan estudou os espaços homogêneos $X^{n}$ com uma ação de um grupo $G$ em $X^{n}$, e a esses associou uma família de referenciais com a propriedade que $G$ age simples e transitivamente nessa família, isto é, a cada par de referenciais existe uma transformação $R \in G$ que manda um referencial no outro. Por exemplo, no espaço Euclidiano $\mathbb{E}^{n}$, a cada ponto $x \in \mathbb{E}^{n}$ podemos associar um referencial de vetores ortonormais $\left\{e_{1}, \cdots, e_{n}\right\}$, ou seja, $\left\langle e_{i}, e_{j}\right\rangle=\delta_{j}^{i}$. Em qualquer sistema de coordenadas, tal referencial está associado a um elemento de $O(n)$, cuja dimensão é $\frac{n(n-1)}{2}$, como a origem de cada referencial depende de $n$ variáveis, o espaço $A S O(n)$ dos referenciais $\left\{x, e_{1}, \cdots, e_{n}\right\}$ tem dimensão $\frac{n(n-1)}{2}+n=\frac{n(n+1)}{2}$. No espaço de referenciais introduziu as fórmulas de derivação

$$
\left\{\begin{array}{l}
d x=\omega^{i} e_{i} \\
d e_{i}=\omega_{j}^{i} e_{j},
\end{array}\right.
$$

e tomando a derivada exterior das fórmulas de derivação obteve as equações de estrutura

$$
\left\{\begin{array}{l}
d \omega^{i}=-\omega_{k}^{i} \wedge \omega^{k} \\
d \omega_{j}^{i}=-\omega_{k}^{i} \wedge \omega_{j}^{k},
\end{array}\right.
$$

em que as formas de conexão $\omega_{j}^{i}$ satisfazem $\omega_{j}^{i}=-\omega_{i}^{j}$. Verifica-se que as equações Gauss e Codazzi seguem das equações de estrutura. A partir dessa construção, Cartan provou que se as formas $\omega^{1}, \cdots, \omega^{r}$ sob certas condições satisfazem as equações de estrutura de um espaço homogêneo, então elas definem uma única família $\Sigma$ de referenciais, a menos da ação de $G$ sob esses referenciais. Tal resultado generaliza o Teorema de Frênet, que diz que uma curva em $\mathbb{E}^{3}$ está determinada por sua curvatura e torção, e o Teorema de Bonnet, que estabelece que uma superfície de $\mathbb{E}^{3}$ fica determinada por sua primeira e segunda forma fundamental.

Na década de 1970, vários pesquisadores, por exemplo Chern, Green, Griffiths e Jensen, reformularam a construção intuitiva de Cartan para um teoria mais ampla e sólida. Um passo conceitual importante para esse desenvolvimento foi dissociar a teoria de fibrados de 
referenciais e conexões para definir referenciais móveis como uma aplicação equivariante da variedade ou do fibrado de jatos no grupo de transformações.

Ao abordar sistemas diferenciais exteriores em uma variedade $\Sigma$, trataremos dos aspectos da teoria de Cartan-Kähler via exemplos provenientes dos referenciais móveis. O objetivo da teoria é garantir a existência de variedades integrais de ideais $\mathcal{I} \subset \Omega^{*}(\Sigma)$. Analisaremos alguns casos particulares de ideais, e justamente os ideais que têm a propriedade de serem fechados por derivação são chamados de sistemas diferenciais exteriores. Duas contribuições centrais de Élie Cartan à teoria são o algoritmo para um dos principais exemplos, a saber sistemas lineares de Pfaff, e o teste de Cartan, que traz um critério prático para um dado plano integral $E$ de um sistema diferencial exterior $\mathcal{I}$ (isto é, $E$ é tal que para cada $\alpha \in \mathcal{I}$, temos $\left.\alpha\right|_{E}=0$ ) ser plano tangente de uma subvariedade integral de $\mathcal{I}$.

Quanto ao teorema de Gauss-Bonnet-Chern, os artigos The Euler number of a Riemann manifold [Allendoerfer, 1940] e On total curvatures of Riemannian manifolds I [Fenchel, 1940], independente um do outro, generalizaram a fórmula de Gauss-Bonnet para variedades mergulhadas no espaço Euclidiano, e o artigo The Gauss-Bonnet Theorem for Riemannian Polyhedra [Carl B. Allendoerfer, 1943] expõe uma generalização para poliedros Riemannianos. Em seu artigo A Simple Intrinsic Proof of the Gauss-Bonnet Formula for Closed Riemannian Manifolds [Chern, 1944] o autor apresenta uma demonstração intrínseca, isto é, independente do ambiente, utilizando-se da teoria de campos vetoriais.

Através das formas de conexão e equações de estrutura de uma variedade $M^{n}$, Chern introduz uma forma $\Omega \in \Omega^{n}(M)$, que generaliza o conceito de curvatura para superfícies, e no caso de $M$ ser fechada, com dimensão par e orientável, vale

$$
\int_{M} \Omega=\chi(M)
$$

em que $\chi(M)$ é a característica de Euler de $M$. A ideia central da prova é observar que a forma $\Omega$ quando levada pelo pull-back para o fibrado unitário $S M$ é exata, e o Teorema do Índice de Hopf da teoria de campos garante a igualdade com a característica de Euler.

No que diz respeito a dissertação em si, os objetos aqui trabalhados são sempre supostos com máxima diferenciabilidade e é adotada a convenção de Einstein para somatórias. 


\section{Geometria Riemanniana via Referenciais Móveis}

\subsection{Sistemas diferenciais exteriores}

Nesta seção estabeleceremos noções básicas sobre sistemas diferenciais exteriores. Desenvolveremos nesse primeiro momento apenas o suficiente para discutir nas próximas seções o método do referencial móvel, e no Capítulo 3 abordaremos o assunto com mais profundidade. No que segue, $\Sigma$ é variedade diferencial de dimensão $n$ e $\Omega^{p}(\Sigma)$ espaço das $p$-formas diferenciais de $M$.

Definição 1.1.1. Seja $\mathcal{I}$ um ideal em $\Omega^{*}(\Sigma)$, dizemos que $\mathcal{I}$ é um ideal diferencial se existe um conjunto $\left\{\omega^{i}\right\}_{i \in I}$ tal que $\mathcal{I}$ é gerado por $\left\{\omega^{i}, d \omega^{i}\right\}_{i \in I}$. Observe que é imediato o fato de $\alpha \in \mathcal{I}$ implicar $d \alpha \in \mathcal{I}$. Um ideal $\mathcal{I}$ no sentido usual será dito ideal algébrico.

Um sistema diferencial exterior com condição de independência em uma variedade $\Sigma$ é o $\operatorname{par}(\mathcal{I}, \Omega)$, em que $\mathcal{I} \subset \Omega^{*}(\Sigma)$ é um ideal diferencial e $\Omega \in \Omega^{n}(\Sigma)$ é uma $n$-forma. Dizemos que $\Omega$ é a condição de independência.

Dizemos que uma subvariedade imersa $i: M^{n} \subset \Sigma$ é uma variedade integral de um sistema diferencial exterior com condição de independência $(\mathcal{I}, \Omega)$ se $i^{*}(\alpha)=0, \forall \alpha \in \mathcal{I}$ e $i^{*}(\Omega) \neq 0$.

Observação 1.1.2. Na definição anterior, se $\tilde{\Omega}=\lambda \Omega$ para $\lambda$ não nulo, então $M$ também é variedade integral de $(\mathcal{I}, \tilde{\Omega})$. Desse modo, a condição de independência será considerada a menos de escalar.

Definição 1.1.3. Dizemos que $E \in G_{n}\left(T_{x} \Sigma\right)$, isto é, $E$ elemento da Grassmanniana de dimensão $n$ em $T_{x} \Sigma$, é um elemento integral de $(\mathcal{I}, \Omega)$ em x se $\left.\Omega\right|_{E} \neq 0$ e $\left.\alpha\right|_{E}=0, \forall \alpha \in \mathcal{I}$. Esse espaço de elementos integrais será denotado $\mathcal{V}_{n}(\mathcal{I}, \Omega)_{x}$.

Na definição anterior, se denotarmos $\mathcal{I}^{n}:=\mathcal{I} \cap \Omega^{n}(\Sigma)$, verificamos imediatamente que $\mathcal{V}_{n}(\mathcal{I}, \Omega)_{x} \subset\left\{E \in G_{n}\left(T_{x} \Sigma\right):\left.\Omega\right|_{E} \neq 0\right.$ e $\left.\left.\alpha\right|_{E}=0, \forall \alpha \in \mathcal{I}^{n}\right\} ;$ reciprocamente, seja $E$ tal 
que $\left.\alpha\right|_{E}=0, \forall \alpha \in \mathcal{I}^{n}$, e suponha que exista $\phi \in \mathcal{I}$, $\operatorname{com} \operatorname{deg}(\phi)=p<n$, tal que $\left.\phi\right|_{E} \neq 0$. Tome $\eta_{0} \in \Omega^{n-p}\left(E^{*}\right)$ de forma que $\left.\phi\right|_{E} \wedge \eta_{0} \neq 0$ e estenda $\eta_{0}$ a $\eta \in \Omega^{n-p}(\Sigma)$. Então $\phi \wedge \eta$ tem grau $n$ e está em $\mathcal{I}$, mas $\left.(\phi \wedge \eta)\right|_{E}=\left.\phi\right|_{E} \wedge \eta_{0} \neq 0$ contradição. Concluímos que $\left.\phi\right|_{E}=0$ para todo $\phi \in \mathcal{I}$ e obtemos a inclusão no outro sentido.

Dizemos que $\mathcal{I}$ um ideal diferencial é um sistema diferencial exterior quando não impomos condição inicial. Note que nesse caso as variedades integrais não possuem dimensão especificadas. Analogamente à Definição 3.1.3 temos $E \in G\left(k, T_{x} \Sigma\right)$ elemento integral se $\left.\alpha\right|_{E}=0, \forall \alpha \in \mathcal{I}$. O conjunto dos elementos integrais de dimensão $k$ nesse caso será denotado $\mathcal{V}_{k}(\mathcal{I})_{x}$.

Notação. Sejam $\theta^{1}, \cdots, \theta^{n} \in \Omega^{*}(M)$. Um ideal diferencial algébrico gerado por $\theta^{i}$ será denotado $\left\{\theta^{1}, \cdots, \theta^{n}\right\}_{\text {alg }}$; quando for fechado por derivação, escreveremos $\left\{\theta^{1}, \cdots, \theta^{n}\right\}_{\text {diff }}$.

Exemplo 1.1.4. Em $\Sigma=\mathbb{R}^{3}$, defina o ideal $\mathcal{I}=\{x d y, d z\}_{d i f f}$. Vamos calcular alguns espaços de elementos integrais. Temos:

$$
\mathcal{V}_{1}(\mathcal{I})_{(1,1,1)}=\left\{\left\{(u, 0,0)_{(1,1,1)}: u \in \mathbb{R}\right\}\right\}
$$

isto é, o conjunto que tem como único elemento o eixo $x$ de $T_{(1,1,1)} \mathbb{R}^{3}$. De fato, estamos procurando retas $r$ em $T_{(1,1,1)} \mathbb{R}^{3}$ tais que $\left.(x d y)_{(1,1,1)}\right|_{r}=0$ e $\left.d z_{(1,1,1)}\right|_{r}=0$. Da primeira condição temos $\left.1 d y\right|_{r}=\left.d y\right|_{r}=0$ e da segunda $\left.d z\right|_{r}=0$, ou seja, a reta deve ser o eixo $x$ de $T_{(1,1,1)} \mathbb{R}^{3}$. Analogamente, $\mathcal{V}_{1}(\mathcal{I})_{(0,0,0)}$ é o conjunto das retas contidas no plano $x y$ em $T_{(0,0,0)} \mathbb{R}^{3}$ e $\mathcal{V}_{2}(\mathcal{I})_{(0,0,0)}$ é o conjunto cujo único elemento é o plano $x y$ de $T_{(0,0,0)} \mathbb{R}^{3}$.

Exemplo 1.1.5. Sejam $M, N$ variedades de dimensão $n$ e $s$, respectivamente, e considere o espaço dos $k$-jatos $J^{k}(M, N)$. Em coordenadas locais $\left(x^{1}, \cdots, x^{n}\right)$ e $\left(u^{1}, \cdots, u^{s}\right)$ que temos um elemento de $J^{k}(M, N)$ é dado por $\left(x^{i}, u^{a}, p_{i}^{a}, p_{i j}^{a}, \cdots, p_{i^{1} \ldots i^{k}}^{a}\right)$, em que $I=$ $\left(i^{1} \cdots i^{k}\right)$ é multi-índice crescente de tamanho $k, k \in\{1, \cdots, \operatorname{dim}(M)\}$. Podemos definir $\Omega=d x^{1} \wedge \cdots \wedge d x^{n}$, e $\mathcal{I}$ como sendo ideal gerado por

$$
\begin{aligned}
& \theta^{a}:=d u^{a}-p_{i}^{a} d x^{i} \\
& \theta_{i}^{a}:=d p_{i}^{a}-p_{i j}^{a} d x^{j} \\
& \vdots \\
& \theta_{i^{1 \ldots i} i^{k-1}}^{a}-p_{i^{1} \ldots i^{k}}^{a} d x^{i^{k}} .
\end{aligned}
$$

Tais formas são ditas formas de contato. Usando a abreviação via multi-índices, as formas de contato são $\theta_{I}^{a}=d p_{I}^{a}-p_{I j}^{a} d x^{j}$. Note que se tivermos outros sistemas de coordenadas 
em $M$ e $N$, as formas de contato nas novas coordenadas serão combinações lineares das anteriores. Logo $\mathcal{I}$ não depende da escolha das coordenadas e o sistema diferencial exterior $(\mathcal{I}, \Omega)$ está definido globalmente. O sistema $(\mathcal{I}, \Omega)$ é dito sistema de contato canônico em $J^{k}(M, N)$.

Um fato acerca das variedades integrais de $(\mathcal{I}, \Omega)$ é que, localmente, essas são os levantamentos de gráficos de $f: M \rightarrow N$,

$$
\Gamma_{f}:=\{(x,(f(x)): x \in M\}
$$

para $J^{k}(M, N)$. Verificando a afirmação anterior: fixadas coordenadas $\left(x^{i}\right)$ e $\left(u^{a}\right)$, temos o levantamento $L: \Gamma_{f} \rightarrow J^{k}(M, N)$, dado por $L\left(x^{i}, f^{a}\left(x^{i}\right)\right)=\left(x^{i}, f^{a}\left(x^{i}\right), \frac{\partial^{|I|} f^{a}}{\partial x^{I}}\left(x^{i}\right)\right)$. Denote $X:=L(U), U \subset M$, e $i: X \rightarrow J^{k}(M, N)$ inclusão, e note que $i^{*}\left(\theta_{I}^{a}\right)=0$, pois $p_{I}^{a}=\frac{\partial^{|I|} f^{a}}{\partial x^{I}}\left(x^{i}\right)$. Reciprocamente se $X$ é variedade integral, temos $x \in X$ é da forma $\left(x^{i}, u\left(x^{i}\right), p_{I}^{a}\left(x^{i}\right)\right)$, uma vez que vale a condição de independência $d x^{1} \wedge \cdots \wedge d x^{n} \neq 0$ em $X$. Logo ser variedade integral significa $i^{*}\left(\theta_{I}^{a}\right)=0$ se, e somente se, $p_{I}^{a}=\frac{\partial^{|I|} u^{a}}{\partial x^{I}}\left(x^{i}\right), I$ multi-índice decrescente, tal que $1 \leq|I| \leq k$, isto é, $X$ é o levantamento do gráfico de $u: U \rightarrow N$.

Observação 1.1.6. Seja $i: M \rightarrow \Sigma$ inclusão. Doravante cometeremos abuso de notação e escreveremos $\theta=0$ e $\Omega \neq 0$ em $M$ para nos referir a $i^{*}(\theta)=0$ e $i^{*}(\Omega) \neq 0$.

\subsection{A forma de Maurer-Cartan}

Nesta seção trataremos da forma de Maurer-Cartan e, a partir dela, buscaremos invariantes geométricos de grupos de Lie matriciais.

Definição 1.2.1. Seja $G$ subgrupo de Lie, $\mathfrak{g}$ sua álgebra de Lie, tal que exista $g: G \rightarrow$ $M_{n}(\mathbb{R})$ homomorfismo sobre a imagem. Definimos a forma de Maurer-Cartan de $G$ com valores em $M_{n}(\mathbb{R})$ por

$$
\begin{aligned}
\omega: G \longrightarrow T M_{n}(\mathbb{R}) & \\
a \longmapsto \omega_{a}: T_{a} G & \longrightarrow T_{g(a)} M_{n}(\mathbb{R}) \\
v & \longmapsto \omega_{a}(v)=g(a)^{-1} d g_{a}(v)
\end{aligned}
$$

Observação 1.2.2. Seja $v \in T_{a} G$, identificando $G$ com sua imagem por $g$ e $T_{g(a)} M_{n}(\mathbb{R}) \cong$ $M_{n}(\mathbb{R})$, segue $d g_{a}(v) \in T_{g(a)} G$, multiplicando a esquerda por $g^{-1}(a)$ temos $\omega_{a}(v)=$ $g^{-1}(a) d g_{a}(v) \in T_{e} G \cong \mathfrak{g}$. 
Exemplo 1.2.3. Considere o grupo de Lie $G=(\mathbb{R},+)$, seja $g: G \rightarrow M_{2}(\mathbb{R})$ dado por:

$$
g(\theta)=\left(\begin{array}{cc}
\cos \theta & -\sin \theta \\
\sin \theta & \cos \theta
\end{array}\right) .
$$

Denotamos $g(G)=S O(2)$, e nesse caso temos

$$
d g=\left(\begin{array}{cc}
-\sin \theta d \theta & -\cos \theta d \theta \\
\cos \theta d \theta & -\sin \theta d \theta
\end{array}\right)
$$

portanto

$$
\omega=g^{-1} d g=\left(\begin{array}{cc}
0 & -d \theta \\
d \theta & 0
\end{array}\right) .
$$

Definição 1.2.4. Dizemos que uma forma $\alpha \in \Omega^{k}(G)$ é invariante à esquerda se para cada $a \in G$ temos $\left(L_{a}\right)^{*} \alpha=\alpha$. Em que $L_{a}: G \rightarrow G$ multiplicação à esquerda.

Lema 1.2.5. A forma $\omega$ da Definição 1.2.1 é invariante à esquerda.

Demonstração. Como $G$ é grupo de matrizes, segue $d\left(L_{a}\right)_{b}(v)=a v$, em que $a, b \in G$ e $v \in T_{b} G$. Do fato que $G$ é homomorfismo temos, para $a \in G$, fixo que $g \circ L_{a}=L_{g(a)} \circ g$; diferenciando em $b$, temos

$$
d g_{a b} \circ d\left(L_{a}\right)_{b}=d\left(L_{g(a)}\right)_{g(b)} \circ d g_{b}=g(a) d g_{b} .
$$

Calculando $L_{a}^{*}(\omega)_{b}$ explicitamente:

$$
\begin{aligned}
L_{a}^{*}(\omega)_{b}(v) & =g(a b)^{-1} d g_{a b}\left(d\left(L_{a}\right)_{b}(v)\right) \\
& \stackrel{1.1}{=} g(b)^{-1} g(a)^{-1} g(a) d g_{b}(v) \\
& =g(b) d g_{b}(v) \\
& =\omega_{b}(v),
\end{aligned}
$$

para $v \in T_{b} G$. Isto é, $L^{*} \omega=\omega$.

Observação 1.2.6. Podemos também definir a forma de Maurer-Cartan para um grupo de Lie qualquer $G$ como a única forma invariante a esquerda com valores em $\mathfrak{g}$ tal que $\omega_{e}$ : $T_{e} G \rightarrow \mathfrak{g}$ é identificação usual. Note que ser invariante significa $\omega_{a b}\left(d\left(L_{a}\right)_{b}(v)\right)=\omega_{b}(v)$. Tomando $b=e$, reduzimos para $\omega_{a}\left(d\left(L_{a}\right)_{e}(v)\right)=\omega_{e}(v)$. Denotando $w=d\left(L_{a}\right)_{e}(v)$, temos $\omega_{a}(w)=\omega_{e}\left(d\left(L_{a}\right)_{e}^{-1}(w)\right)$, como $d\left(L_{a}\right)_{e}^{-1}=d\left(L_{a^{-1}}\right)_{a}$, segue

$$
\omega_{a}(w)=\omega_{e}\left(d\left(L_{a^{-1}}\right)_{a}(w)\right)
$$


isto é, todos os valores de $\omega_{a}$ estão determinados por $\omega_{e}$. A Observação 1.2.2 garante que as definições de $\omega$ coincidem no caso de grupos de matrizes, já que $\omega$ da primeira definição toma valores em $\mathfrak{g}$, é invariante pelo Lema 1.2 .5 e, identificando $G \cong g(G), \omega_{e}=I d_{T_{e} G}$.

Definição 1.2.7. Sejam $M$ variedade diferenciável, $G$ grupo de Lie, $H \leq G$, e $f: M \rightarrow \frac{G}{H}$. Dizemos que $F$ é levantamento de $f$ se o seguinte diagrama é comutativo:

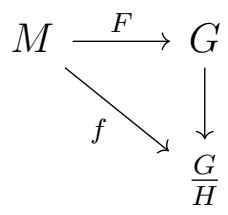

Observação 1.2.8. Seja $A \in G$, denotamos $\bar{A}$ a classe de $A$ em $G / H$. No contexto da definição anterior, se $\widetilde{F}$ for outro levantamento, então para cada $x \in M$ temos $f(x)=$ $\overline{F(x)}=\overline{\widetilde{F}(x)}$, isto é, existe $a(x) \in H$ tal que $\widetilde{F}(x)=F(x) a(x)$. A forma de Maurer-Cartan de um grupo de matrizes satisfaz a seguinte relação:

$$
\begin{aligned}
\widetilde{F}^{*} \omega & =\left(\left(F^{*} \omega\right) a\right)^{-1} d\left(\left(F^{*} \omega\right) a\right) \\
& =a^{-1}\left(F^{*} \omega\right)^{-1}\left(d\left(F^{*} \omega\right) a+\left(F^{*} \omega\right) d a\right) \\
& =a^{-1}\left(F^{*} \omega\right) a+a^{-1} d a .
\end{aligned}
$$

Para $G$ grupo de Lie qualquer, obtemos a generalização $\widetilde{F}^{*} \omega=A d_{a^{-1}}\left(F^{*} \omega\right)+a^{*} \omega$.

Lema 1.2.9. Sejam G grupo de Lie de matrizes e $\omega$ forma de Maurer-Cartan, então

$$
d \omega=-\omega \wedge \omega
$$

Tal igualdade é dita equação de Maurer-Cartan.

Demonstração. Temos que $\omega=g^{-1} d g$, de onde $d \omega=d\left(g^{-1}\right) \wedge d g+g^{-1} \wedge d(d g)$; o segundo termo é nulo devido a $d^{2} \equiv 0$, e basta computar $d\left(g^{-1}\right)$, isto é,

$$
0=d(e)=d\left(g^{-1} g\right)=d\left(g^{-1}\right) g+g^{-1} d g
$$

isolando $d\left(g^{-1}\right)$, segue $d \omega=-g^{-1}(d g) g^{-1} \wedge d g=-g^{-1} d g \wedge g^{-1} d g=-\omega \wedge \omega$.

Observação 1.2.10. Se $\omega$ e $\theta$ são 1-formas com valores em $\mathfrak{g}$, definimos a 2 -forma $[\omega, \theta]$ com valores em $\mathfrak{g}$, por

$$
[\omega, \theta](X, Y)=[\omega(X), \theta(Y)]+[\omega(Y), \theta(X)]
$$

Com essa definição a equação de Maurer-Cartan para um grupo de Lie qualquer torna-se

$$
d \omega=-\frac{1}{2}[\omega, \omega]
$$


Teorema 1.2.11. Seja $G$ um grupo de Lie de matrizes com álgebra de Lie $\mathfrak{g}$ e forma de Maurer-Cartan $\omega$. Sejam $M$ variedade diferenciável e $\phi$ 1-forma com valores em $\mathfrak{g}$ satisfazendo $d \phi=-\phi \wedge \phi$. Então, para cada $x \in M$, existem uma vizinhança $U$ e uma aplicação $f: U \rightarrow G$ tal que $f^{*} \omega=\phi$. Além disso, se duas aplicações $f_{1}, f_{2}$ satisfazem a condição, então $f_{1}=L_{a} \circ f_{2}$ para algum $a \in G$.

Demonstração. Defina $\Sigma=M \times G$ e considere as projeções usuais $\pi: \Sigma \rightarrow M$ e $\rho: \Sigma \rightarrow G$. Considere $\theta=\pi^{*} \phi-\rho^{*} \omega$. Seja $I \subset T^{*} \Sigma$ subfibrado gerado por $\theta_{j}^{i}$, em que $\theta=\left(\theta_{j}^{i}\right)$.

Afirmação I: $\operatorname{posto}(I)=\operatorname{dim}(G)$.

De fato, fixemos $(x, g) \in \Sigma$, mostremos inicialmente que $\theta_{(x, g)}$ é sobrejetora. Seja $X \in \mathfrak{g}$, $X$ é invariante à esquerda, em particular $d\left(L_{g}\right)_{e}\left(X_{e}\right)=X_{g}$, assim

$$
\theta_{(x, g)}\left(0,-X_{g}\right)=\left(\pi^{*} \phi\right)_{(x, g)}\left(0,-X_{g}\right)-\left(\rho^{*} \omega\right)_{(x, g)}\left(0,-d\left(L_{g}\right)_{e}\left(X_{e}\right)\right) .
$$

Podemos escrever o primeiro termo como $\phi_{x}\left(d \pi_{(x, g)}\left(0,-X_{g}\right)\right)$. Identificando $T_{(x, g)} \Sigma \cong$ $T_{x} M \times T_{g} G$, segue que $d \pi$ é projeção na primeira coordenada, logo obtemos o vetor nulo. Para o segundo termo: $\left(\rho^{*} \omega\right)_{(x, g)}\left(0, d\left(L_{g}\right)_{e}\left(X_{e}\right)\right)=\omega_{g}\left(d\left(L_{g}\right)_{e}\left(X_{e}\right)\right)=\omega_{e}\left(X_{e}\right)$, pois $\omega$ é invariante à esquerda, e por definição, $\omega_{e}\left(X_{e}\right)=X$. Pelo teorema do núcleo e da imagem, $\operatorname{dim}(\Sigma)=\operatorname{dim}\left(\operatorname{ker}\left(\theta_{(x, g)}\right)\right)+\operatorname{dim}\left(\operatorname{Im}\left(\theta_{(x, g)}\right)\right)$. Como $\theta_{(x, g)}$ é sobrejetora, segue

$$
\operatorname{dim}\left(\operatorname{Im}\left(\theta_{(x, g)}\right)\right)=\operatorname{dim}(\mathfrak{g})=\operatorname{dim}(G),
$$

isto é, $\operatorname{dim}\left(\operatorname{ker}\left(\theta_{(x, g)}\right)\right)=\operatorname{dim}(\Sigma)-\operatorname{dim}(G)$, como o posto $(I)$ é o complementar da dimensão do núcleo de $\theta$, segue posto $(I)=\operatorname{dim}(\Sigma)-\operatorname{dim}\left(\operatorname{ker}\left(\theta_{(x, g)}\right)\right)=\operatorname{dim}\left(\operatorname{Im}\left(\theta_{(x, g)}\right)\right)=\operatorname{dim}(G)$. Precisamos para completar a prova a seguinte versão do teorema de Frobenius:

Teorema 1.2.12. (Frobenius). Sejam $\Sigma^{m}$ uma variedade diferenciável e $J \subset T^{*} \Sigma o$ ideal diferencial gerado por 1-formas $\left\{\theta_{1}, \cdots, \theta_{m-n}\right\}$. Se $J=\left\{\theta_{1}, \cdots, \theta_{m-n}\right\}_{\text {alg }}$, então para cada $p \in \Sigma$ existe uma única subvariedade integral de $J$, de dimensão n. Mais ainda, em uma vizinhança de $p$ existe sistema de coordenadas $\left(x^{1}, \cdots, x^{m}\right)$ tal que $J=$ $\left\{d x^{1}, \cdots, d x^{m-n}\right\}_{a l g}$.

Demonstração. [Thomas A. Ivey, 2003, pg. 10] e [Lee, 2012, cap. 19] 
Afirmação II: $I=\left\{\left(\theta_{i}^{j}\right)\right\}_{\text {alg }}$. De fato,

$$
\begin{aligned}
d \theta & =\pi^{*}(d \phi)-\rho^{*}(d \omega) \\
& =\pi^{*}(-\phi \wedge \phi)-\rho^{*}(-\omega \wedge \omega) \\
& =-\pi^{*} \phi \wedge \pi^{*} \phi+\rho^{*} \omega \wedge \rho^{*} \omega \\
& =-\pi^{*} \phi \wedge \pi^{*} \phi+\left(\pi^{*} \phi-\theta\right) \wedge\left(\pi^{*} \phi-\theta\right) \\
& =-\pi^{*} \phi \wedge \pi^{*} \phi+\pi^{*} \phi \wedge \pi^{*} \phi-\pi^{*} \phi \wedge \theta-\theta \wedge \pi^{*} \phi+\theta \wedge \theta \\
& =\theta \wedge \theta .
\end{aligned}
$$

No computo acima: a $4^{\mathrm{a}}$ igualdade segue da definição de $\theta$, e na $5^{\mathrm{a}}$ igualdade, os dois primeiros termos cancelam-se e os dois seguintes cancelam-se devido a $\pi^{*} \phi \wedge \theta=-\theta \wedge \pi^{*} \phi$. Portanto $I$ é fechado por derivação, isto é, $I=\left\{\left(\theta_{i}^{j}\right)\right\}_{\text {alg }}$.

Finalmente, pelo Teorema 1.2 .12 e a afirmação anterior, para cada $(x, g) \in \Sigma$ existe $S$ subvariedade integral de $I$, devido a Afirmação $\mathrm{I}, \operatorname{dim}(S)=\operatorname{dim}(\Sigma)-\operatorname{dim}(G)=\operatorname{dim}(M)$, e podemos tomar $S$ como sendo localmente o gráfico de $f: U \subset M \rightarrow G$. Defina $\Phi: U \rightarrow \Sigma, \Phi(x)=(x, f(x))$ e denote $i: S \rightarrow \Sigma$ inclusão; temos que $S$ integral implica em $i^{*}(\theta) \equiv 0 ; \operatorname{logo}$, em $U, 0=\Phi^{*}(\theta)=\Phi^{*}\left(\pi^{*} \phi-\rho^{*} \omega\right)$, ou seja,

$$
\Phi^{*}\left(\pi^{*} \phi\right)=\Phi^{*}\left(\rho^{*} \omega\right)
$$

Como $\pi \circ \Phi=I d$ e $\rho \circ \Phi=f$, temos $\phi=f^{*} \omega$.

Resta provar a unicidade: sejam $f_{1}, f_{2}$ tais que $\phi=f_{1}^{*}(\omega)=f_{2}^{*}(\omega)$, e fixe $\left(x, f_{1}(x)\right) \in \Sigma$. Denote $g=f_{1}(x)$ e $a=g\left(f_{2}(x)\right)^{-1}$. Defina $f: U \rightarrow \Sigma$ por $f(y)=L_{a}\left(f_{2}(y)\right)$. Note que $f$ satisfaz $f(x)=a f_{2}(x)=g\left(f_{2}(x)\right)^{-1} f_{2}(x)=g$ e $f^{*}(\omega)=\left(f_{2}\right)^{*}\left(L_{a}^{*} \omega\right)$. Novamente usando o fato de $\omega$ ser invariante à esquerda, $f^{*}(\omega)=f_{2}^{*}(\omega)=\phi$, pela unicidade do Teorema 1.2.12, segue $f=f_{1}$, isto é, $f_{1}=L_{a} \circ f_{2}$.

Observação 1.2.13. No contexto anterior, se $M$ é conexa e simplesmente conexa, então $f$ pode ser estendida para toda $M$.

Exemplo 1.2.14. Podemos aplicar o Teorema 1.2.11 para estudar curvas no plano euclidiano.

Considere o levantamento de $c: \mathbb{R} \rightarrow \mathbb{E}^{2}, c$ regular e parametrizada pelo comprimento de 
arco. Seja $C: \mathbb{R} \rightarrow A S O(2)$ dada por:

$$
C(t)=\left(\begin{array}{ccc}
1 & 0 & 0 \\
c_{1}(t) & e_{1}^{1}(t) & e_{2}^{1}(t) \\
c_{2}(t) & e_{1}^{2}(t) & e_{2}^{2}(t)
\end{array}\right)
$$

Podemos esquematizar:

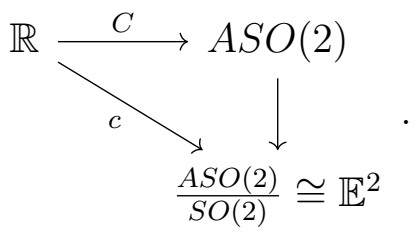

Parametrizando $A S O(2)$ por $g: \mathbb{R} \times \mathbb{E}^{2} \rightarrow A S O(2)$,

$$
g(\theta, x, y)=\left(\begin{array}{ccc}
1 & 0 & 0 \\
x & \cos \theta & -\sin \theta \\
y & \sin \theta & \cos \theta
\end{array}\right)
$$

temos

$$
d g=\left(\begin{array}{ccc}
0 & 0 & 0 \\
d x & -\sin \theta d \theta & -\cos \theta d \theta \\
d y & \cos \theta d \theta & -\sin \theta d \theta
\end{array}\right) \text { e } \omega=g^{-1} d g=\left(\begin{array}{ccc}
0 & 0 & 0 \\
\cos \theta d x+\sin \theta d y & 0 & -d \theta \\
-\sin \theta d x+\cos \theta d y & d \theta & 0
\end{array}\right) .
$$

Para cada $t \in \mathbb{R}, c^{\prime}(t)=(\cos (\theta(t)), \sin (\theta(t)))$. Assim, computando $C^{*} \omega$ :

Primeira coluna:

$$
\left(c^{*}\left(\begin{array}{c}
\cos \theta d x+\sin \theta d y \\
-\sin \theta d x+\cos \theta d y
\end{array}\right)\right)_{t}=\left(\begin{array}{c}
\cos (\theta(t)) \cos (\theta(t))+\sin (\theta(t)) \sin (\theta(t)) \\
-\sin (\theta(t)) \cos (\theta(t))+\cos (\theta(t)) \sin (\theta(t))
\end{array}\right)_{t}=\left(\begin{array}{l}
1 \\
0
\end{array}\right)_{t} .
$$

Para segunda e terceira coluna, basta observar que, para cada $t \in \mathbb{R}, e_{1}(t)^{\prime}=k(t) e_{2}$, isto é, $\cos (\theta(t)) \theta^{\prime}(t)=k(t) \cos (t)$ e $-\sin (\theta(t)) \theta^{\prime}(t)=-k(t) \sin (\theta(t))$, logo $\theta^{\prime}(t)=k(t)$, portanto

$$
\left(\left(\begin{array}{ll}
e_{1}(t) & e_{2}(t)
\end{array}\right)^{*}\left(\begin{array}{cc}
0 & -d \theta \\
d \theta & 0
\end{array}\right)\right)_{t}=\left(\begin{array}{cc}
0 & -k(t) d t \\
k(t) d t & 0
\end{array}\right)
$$

Seja $\tilde{c}: \mathbb{R} \rightarrow \mathbb{E}^{2}$ regular e parametrizada pelo comprimento de arco tal que $k(t)=\tilde{k}(t)$, para cada $t$ real. Denote $C_{1}=C$ e $C_{2}$ o levantamento de $\tilde{c}$. Segue

$$
C_{1}^{*} \omega=C_{2}^{*} \omega=\left(\begin{array}{ccc}
0 & 0 & 0 \\
1 & 0 & -k(t) d t \\
0 & k(t) d t & 0
\end{array}\right)
$$


Do Teorema 1.2.11 $C_{1}=L_{a} \circ C_{2}$, isto é,

$$
\left(\begin{array}{cc}
1 & 0 \\
c(t) & \left(\begin{array}{ll}
e_{1} & e_{2}
\end{array}\right)
\end{array}\right)=\overbrace{\left(\begin{array}{ll}
1 & 0 \\
x & R
\end{array}\right)}^{a}\left(\begin{array}{ccc}
1 & 0 \\
\tilde{c}(t) & \left(\begin{array}{cc}
\tilde{e}_{1}(t) & e_{2}(t)
\end{array}\right)
\end{array}\right),
$$

em particular, $c(t)=x+R \tilde{c}(t)$; em outras palavras $c$ e $\tilde{c}$ são congruentes.

\subsection{Curvas e superfícies via referenciais móveis}

Nesta seção estudaremos o espaço de referenciais ao longo de curvas e superfícies. Esse espaço está contido em um certo grupo de Lie e podemos considerar a restrição da forma de Maurer-Cartan para buscar invariantes geométricos. No que segue, dedicar-nos-emos a formalizar essa ideia.

Seja $\theta: S O(n) \times \mathbb{E}^{n} \rightarrow \mathbb{E}^{n}$, dada por $\theta(R, t)=R t$. Considere o produto semidireto $A S O(n)=\mathbb{E}^{n} \rtimes_{\theta} S O(n)$. O espaço $A S O(n)$ é grupo de Lie, com álgebra de Lie $\mathfrak{a} \mathfrak{s o}(\mathfrak{n})=\mathfrak{s o}(\mathfrak{n}) \times \mathbb{E}^{n}$. Considere a forma de Maurer-Cartan $\omega \in \Omega^{1}(A S O(n), \mathfrak{a s o}(\mathfrak{n}))$. Como $\mathfrak{s o}(\mathfrak{n})$ é o conjunto das matrizes anti-simétricas, temos

$$
\left.\omega=\left(\begin{array}{c}
0 \\
\omega^{1} \\
\vdots \\
\omega^{n}
\end{array}\right)\left(\begin{array}{cccc}
0 & \omega_{2}^{1} & \cdots & \omega_{n}^{1} \\
-\omega_{2}^{1} & 0 & \ddots & \vdots \\
\vdots & \ddots & 0 & \omega_{n}^{n-1} \\
-\omega_{n}^{1} & \cdots & -\omega_{n}^{n-1} & 0
\end{array}\right)\right)
$$

Do fato de $\omega$ ser invariante à esquerda, temos que $\omega^{i}$ e $\omega_{j}^{i}$ são também invariantes. Representando $A S O(n)$ com a aplicação $\left(x,\left(e_{1} \cdots e_{n}\right)\right) \in A S O(n)$,

$$
\left(x,\left(e_{1} \cdots e_{n}\right)\right) \mapsto \underbrace{\left(\begin{array}{cc}
1 & 0 \\
x & \left(e_{1} \cdots e_{n}\right)
\end{array}\right)}_{g} \in G L_{n}(\mathbb{R})
$$

temos $g \omega=d g$; em blocos:

$$
\left(e_{1} \cdots e_{n}\right)\left(\begin{array}{c}
\omega^{1} \\
\vdots \\
\omega^{n}
\end{array}\right)=d x
$$




$$
\left(e_{1} \cdots e_{n}\right)\left(\begin{array}{cccc}
0 & \omega_{2}^{1} & \cdots & \omega_{n}^{1} \\
-\omega_{2}^{1} & 0 & \ddots & \vdots \\
\vdots & \ddots & 0 & \omega_{n}^{n-1} \\
-\omega_{n}^{1} & \cdots & -\omega_{n}^{n-1} & 0
\end{array}\right)=d\left(\begin{array}{lll}
e_{1} & \cdots & e_{n}
\end{array}\right)
$$

De (1.4) temos $d x=\omega^{i} e_{i}$ e (1.5) implica em $d e_{j}=\omega_{j}^{i} e_{i}, \operatorname{com} \omega_{j}^{i}=-\omega_{i}^{j}$.

Proposição 1.3.1. No contexto anterior, $\omega^{i}$ são formas semi-básicas.

Demonstração. Sejam $g \in A S O(n)$ e $X \in T_{g} A S O(n)$ vertical. Tome

$$
C(t)=\left(c(t), e_{1}(t), \cdots, e_{n}(t)\right)
$$

$C(0)=g, C^{\prime}(0)=X$ e $C$ com imagem contida na fibra passando por $g$. Do fato que $C$ está contida em uma fibra $c \equiv g, c^{\prime}(0)=\frac{d x}{d t}=0$. de (1.4) temos $0=\omega^{i}\left(C^{\prime}(0)\right) e_{i}(0)$. Como $e_{i}(0)$ são linearmente independentes, $\omega^{i}(X)=0$, para $i=1, \cdots n$.

Exemplo 1.3.2. (Invariantes de uma curva). Seja $c: \mathbb{R} \rightarrow \mathbb{E}^{n}$, em que $c$ está parametrizada pelo comprimento de arco e $\left\{c^{(1)}(t), \cdots, c^{(n-1)}(t)\right\}$ é um conjunto linearmente independente. Podemos aplicar o processo de Gram-Schmidt definindo $e_{1}(t)=c^{\prime}(t)$, $e_{2}(t)=\frac{c^{(2)}(t)}{\left\|c^{(2)}(t)\right\|}$, e indutivamente, $\tilde{e}_{j}(t)=c^{(j)}-\sum_{i=1}^{j-1}\left\langle c^{(j)}(t), e_{i}(t)\right\rangle e_{i}(t)$ e $e_{j}(t)=\frac{\tilde{e}_{j}(t)}{\left\|\tilde{e}_{j}(t)\right\|}$. Fica determinado base positivamente orientada $\left\{e_{1}(t), \cdots, e_{n}(t)\right\}$ de $T_{c(t)} \mathbb{E}^{n}$.

Usando a forma de Maurer-Cartan:

$$
d\left(c(t), e_{1}(t), \cdots, e_{n}(t)\right)=\left(c(t), e_{1}(t), \cdots, e_{n}(t)\right)\left(\begin{array}{cc}
0 & 0 \\
c^{*}\left(\omega^{i}\right)_{t} & c^{*}\left(\omega_{j}^{i}\right)_{t}
\end{array}\right)
$$

Da primeira coluna $e_{1}(t)=c^{\prime}(t)=\omega^{i}\left(c^{\prime}(t)\right)_{c(t)} e_{i}, \operatorname{logo} \omega^{1}\left(c^{\prime}(t)\right)_{c(t)}=1$ e $\omega^{i}\left(c^{\prime}(t)\right)_{c(t)}=0$ para $i \in\{1, \cdots, n\}$. Observe que $\omega^{1}\left(c^{\prime}(t) s\right)_{c(t)}=s \omega^{1}\left(c^{\prime}(t)\right)_{c(t)}=s=d t(s), \operatorname{logo} c^{*} \omega^{1}=d t$ e $c^{*} \omega^{i}=0$ para $i \in\{2, \cdots, n\}$.

Para $j \geq 2$, temos $e_{j}^{\prime}(t)=\omega_{j}^{i}\left(c^{\prime}(t)\right)_{c(t)} e_{i}(t)$ mas por, construção, $e_{j}^{\prime}(t) \in \operatorname{span}\left\{e_{1}(t), \cdots\right.$, $\left.e_{j-1}(t)\right\}$, então $\omega_{j}^{j}\left(c^{\prime}(t)\right)_{c(t)}=\omega_{j}^{j+2}\left(c^{\prime}(t)\right)_{c(t)}=\cdots=\omega_{j}^{n}\left(c^{\prime}(t)\right)_{c(t)}=0$, por anti-simetria

$$
c^{*}\left(\omega_{j}^{i}\right)_{t}=\left(\begin{array}{ccccc}
0 & c^{*}\left(\omega_{2}^{1}\right)_{t} & 0 & \cdots & 0 \\
-c^{*}\left(\omega_{2}^{1}\right)_{t} & 0 & c^{*}\left(\omega_{3}^{2}\right)_{t} & \cdots & \vdots \\
0 & -c^{*}\left(\omega_{3}^{2}\right)_{t} & 0 & \ldots & \vdots \\
\vdots & \vdots & \vdots & \ddots & c^{*}\left(\omega_{n}^{n-1}\right) \\
0 & \cdots & \ldots & -c^{*}\left(\omega_{n}^{n-1}\right) & 0
\end{array}\right)
$$


e (1.6) torna-se

$$
\left\{\begin{array}{l}
c^{\prime}(t)=e_{1}(t) \\
e_{1}^{\prime}(t)=-\omega_{2}^{1}\left(c^{\prime}(t)\right)_{c(t)} e_{1}(t) \\
e_{j}^{\prime}(t)=\omega_{j}^{j-1}\left(c^{\prime}(t)\right)_{c(t)} e_{j-1}(t)-\omega_{j+1}^{j}\left(c^{\prime}(t)\right)_{c(t)} e_{j+1}, \text { para } j \in\{2, \cdots, n-1\} \\
e_{n}^{\prime}(t)=\omega_{n}^{n-1}\left(c^{\prime}(t)\right) e_{n-1}(t) .
\end{array}\right.
$$

Defina $k_{i}(t):=\omega_{i}^{i-1}\left(c^{\prime}(t)\right)$. Então

$$
d\left(e_{1}(t), \cdots, e_{n}(t)\right)=\left(e_{1}(t), \cdots, e_{n}(t)\right)\left(\begin{array}{ccccc}
0 & k_{1} & 0 & \cdots & 0 \\
-k_{1} & 0 & k_{2} & \cdots & \vdots \\
0 & -k_{2} & 0 & \cdots & \vdots \\
\vdots & \vdots & \vdots & \ddots & k_{n} \\
0 & \cdots & \cdots & -k_{n} & 0
\end{array}\right)
$$

Por outro lado, sejam $k_{i}: \mathbb{R} \rightarrow \mathbb{R}$ funções dadas. $\operatorname{Em} \mathbb{R} \times A S O(n)$, considere o ideal deferencial $\mathcal{I}$ gerado por $\left\{\omega^{1}-d t, \omega^{2}, \cdots, \omega^{n}, \omega_{i}^{i-1}-k_{i} \omega_{1}, \omega_{j}^{i}\right\}_{i<j}$. De $d \omega=-\omega \wedge \omega$ segue facilmente que $\mathcal{I}$ é algébrico e, como as formas são linearmente independentes, o posto de $\mathcal{I}$ é $\frac{n(n+1)}{2}$. Pelo teorema de Frobenius, para cada $(s, g) \in \mathbb{R} \times A S O(n)$, existe uma subvaridade $M$ de dimensão 1 passando por $(s, g)$. Parametrizando $M$ por $C: \mathbb{R} \rightarrow A S O(n)$, temos $C^{*}\left(\omega^{1}\right)=d t, C^{*}\left(\omega_{i}^{i-1}\right)=k_{i} d t$ e $k_{i}$ são os invariantes de $c(t):=\pi_{\mathbb{E}^{n}}(C(t))$.

Observação 1.3.3. Para dimensões baixas recuperamos os casos clássicos. Se $n=2$, denote $k=k_{1}, e_{1}=T$ e $e_{2}=N$. Então

$$
d\left(\begin{array}{l}
T \\
N
\end{array}\right)=\left(\begin{array}{ll}
T & N
\end{array}\right)\left(\begin{array}{cc}
0 & k \\
-k & 0
\end{array}\right) .
$$

Para $n=3$, denote $k=k_{1}, \tau=k_{2}, e_{1}=T, e_{2}=N$ e $e_{3}=B$, então

$$
d\left(\begin{array}{c}
T \\
N \\
B
\end{array}\right)=\left(\begin{array}{lll}
T & N & B
\end{array}\right)\left(\begin{array}{ccc}
0 & k & 0 \\
-k & 0 & \tau \\
0 & -\tau & 0
\end{array}\right)
$$

Exemplo 1.3.4. Superfícies $M^{n}$ em $\mathbb{E}^{n+s}$. Seja $\mathcal{F}^{1} \rightarrow M$ o subfibrado de $\left.A S O(n+s)\right|_{M}$ dos referenciais adaptados, isto é, $\left(x, e^{1}, \cdots, e^{n+s}\right) \in \mathcal{F}^{1}$ se $T_{p} M=\operatorname{span}\left\{e^{1}, \cdots, e^{n}\right\}$. Para fixar notação, escrevemos $1 \leq i, j \leq n$ e $n+1 \leq a, b \leq n+s$. Considere $i$ : 
$\mathcal{F}^{1} \rightarrow A S O(n+s)$ a inclusão. Como $T_{p} M$ é gerado por $\left\{e^{1}, \cdots, e^{n}\right\}$, então a forma de Maurer-Cartan $\omega$ satisfaz $i^{*} \omega^{a}=0$. De (1.3), temos $d \omega^{a}=-\omega_{i}^{a} \wedge \omega^{i}-\omega_{b}^{a} \wedge \omega^{b}$ e segue

$$
\begin{aligned}
0 & =d i^{*}\left(\omega^{a}\right) \\
& =i^{*}\left(d \omega^{a}\right) \\
& =-i^{*}(\omega_{i}^{a} \wedge \omega^{i}-\underbrace{\omega_{b}^{a} \wedge \omega^{b}}_{0 \mathrm{em} \mathcal{F}^{1}}) \\
& =-i^{*}\left(\omega_{i}^{a} \wedge \omega^{i}\right),
\end{aligned}
$$

o conjunto $\left\{\omega^{1}, \cdots, \omega^{n}\right\}$ é base de $T_{p}^{*} M$. Em particular, $i^{*}\left(\omega^{i}\right)$ é linearmente independente. Então existem $h_{i j}^{a}: \mathcal{F}^{1} \rightarrow \mathbb{R}$, simétricas nos índices inferiores, tais que $\omega_{i}^{a}=h_{i j}^{a} \omega^{j}$. Para tal existência, usamos o

Lema 1.3.5. (Cartan). Sejam $v^{1}, \cdots, v^{k}$ linearmente independentes em um espaço vetorial $V$ e $w^{1}, \cdots, w^{k}$ elementos de $V$ quaisquer tais que $\sum_{i=1}^{k} v^{i} \wedge w^{i}=0$, então existem $h_{i j} \in \mathbb{R}$ tais que $w^{i}=h_{i j} v^{k}$, em que $h_{i j}=h_{j i}$.

Demonstração. [Sternberg, 1964, p. 18]

Seja $f=\left(\begin{array}{ccc}1 & 0 & 0 \\ x^{i} & e^{i} & 0 \\ x^{a} & 0 & e^{a}\end{array}\right) \in \mathcal{F}^{1}$. As rotações em $\mathcal{F}_{x}^{1}$ são dadas por

$$
R=\left(\begin{array}{ccc}
1 & 0 & 0 \\
0 & g_{j}^{i} & 0 \\
0 & 0 & u_{b}^{a}
\end{array}\right)
$$

em que $\left(g_{j}^{i}\right) \in S O(n)$ e $\left(u_{b}^{a}\right) \in S O(s)$. Outro elemento $\tilde{f} \in \mathcal{F}_{x}^{1}$ é da forma $f R$, assim

$$
\begin{aligned}
\widetilde{\omega} & =\tilde{f}^{-1} d \tilde{f} \\
& =R^{-1} f^{-1} d(f R) \\
& =R^{-1} f^{-1}(d f R+f d R) \\
& =R^{-1} \omega R+R^{-1} d R .
\end{aligned}
$$


Em particular

$$
\left\{\begin{array}{l}
\widetilde{\omega}^{i}=\left(g^{-1}\right)_{j}^{i} \omega^{j} \\
\widetilde{\omega}_{j}^{a}=g_{j}^{i}\left(u^{-1}\right)_{b}^{a} \omega_{i}^{b} \\
\widetilde{h}_{i j}^{a}=\left(u^{-1}\right)_{b}^{a} g_{i}^{k} g_{j}^{l} h_{k l}^{b} \\
\widetilde{e}^{a}=u_{b}^{a} e^{b} .
\end{array}\right.
$$

Defina $\widetilde{I I}:=\sum_{a} \omega_{j}^{a} \omega^{j} \otimes e^{a}=\sum_{a} h_{i j}^{a} \omega^{i} \omega^{j} \otimes e^{a} \in \Gamma\left(\mathcal{F}^{1}, \pi^{*}\left(S^{2}\left(T^{*} M \otimes N M\right)\right)\right)$, de (1.7) temos que $\widetilde{I I}$ é constante em $\mathcal{F}_{x}$. Tome $s: M \rightarrow \mathcal{F}^{1}$ seção local, definimos

Definição 1.3.6. $I I=s^{*}(\widetilde{I I}) \in \Gamma\left(M, S^{2}\left(T^{*} M \otimes N M\right)\right)$ é a segunda forma fundamental de $M$.

Como $\widetilde{I I}$ é constante em $\mathcal{F}_{x}$, isto é, básica, então $I I$ não depende de $s$. Analogamente a $I I$, podemos definir $\widetilde{I}=\sum_{i}=\omega^{i} \omega^{i} \in \Gamma\left(\mathcal{F}^{1}, \pi^{*}\left(S^{2} T^{*} M\right)\right)$. Novamente, de (1.7), $\widetilde{I}$ é constante em $\mathcal{F}_{x}^{1}$, logo básica, então, para qualquer seção local $s: M \rightarrow \mathcal{F}^{1}, I=s^{*}(\widetilde{I}) \in$ $\Gamma\left(M, S^{2}\left(T^{*} M\right)\right.$ é invariante diferencial. I é dita métrica Riemanniana em $M$ ou primeira forma fundamental de $M$.

Para o caso $n=2$ e $s=1$, omitindo o pull-back de $i: \mathcal{F}^{1} \rightarrow A S O(3)$, segue

$$
\left\{\begin{array}{l}
\omega_{1}^{3}=h_{11} \omega^{1}+h_{12} \omega^{2} \\
\omega_{2}^{3}=h_{21} \omega^{1}+h_{22} \omega^{2}
\end{array}\right.
$$

ou equivalentemente, $\left(\begin{array}{l}\omega_{1}^{3} \\ \omega_{2}^{3}\end{array}\right)=\underbrace{\left(\begin{array}{ll}h_{11} & h_{12} \\ h_{12} & h_{22}\end{array}\right)}_{h}\left(\begin{array}{l}\omega^{1} \\ \omega^{2}\end{array}\right)$. Da terceira linha de (1.7), outro elemento $\widetilde{h}$ da fibra $\mathcal{F}_{x}^{1}$ satisfaz $\widetilde{h}=g^{-1} h g$, em que $g \in S O(2)$, portanto as funções $K=\operatorname{det}(h)$ e $H=\frac{1}{2} \operatorname{tr}(h)$ são constantes em $\mathcal{F}_{x}^{1}$. $K$ é dita curvatura Gaussiana de $M$ e $H$ curvatura média de $M$. Observe também que $I I=\omega_{1}^{3} \omega^{1}+\omega_{2}^{3} \omega^{2}=$ $h_{11}\left(\omega^{1}\right)^{2}+2 h_{12} \omega^{1} \omega^{2}+h_{22}\left(\omega^{2}\right)^{2}$. 
De (1.3), entrada $(1,2)$,

$$
\begin{aligned}
d \omega_{2}^{1} & =-\underbrace{\omega_{1}^{1} \wedge \omega_{2}^{1}}_{0}-\underbrace{\omega_{2}^{1} \wedge \omega_{2}^{2}}_{0}-\omega_{3}^{1} \wedge \omega_{2}^{3} \\
& =-\omega_{3}^{1} \wedge \omega_{2}^{3} \\
& =\omega_{1}^{3} \wedge \omega_{2}^{3} \\
& =\left(h_{11} \omega^{1}+h_{12} \omega^{2}\right) \wedge\left(h_{21} \omega^{1}+h_{22} \omega^{2}\right) \\
& =\left(h_{11} h_{22}-\left(h_{12}\right)^{2}\right) \omega^{1} \wedge \omega^{2} \\
& =K \omega^{1} \wedge \omega^{2} .
\end{aligned}
$$

Com o que definimos no exemplos acima podemos demonstrar o seguinte teorema

Teorema 1.3.7. (Egregium de Gauss). A curvatura Gaussiana de uma superfície $M^{2}$ depende apenas da métrica Riemanniana $I$.

Demonstração. Seja $f: U \subset \mathbb{R}^{2} \rightarrow M^{2}$ uma parametrização local, e considere um levantamento de $f F: U \rightarrow \mathcal{F}^{1}$. Tome $X^{i}$ tais que $f_{*}\left(X^{i}\right)=e^{i}, i=1,2$ e $g=f^{*}(I)$, de modo que $g\left(X^{i}, X^{j}\right)=I\left(f_{*}\left(X^{i}\right), f_{*}\left(X^{j}\right)\right)=I\left(e^{i}, e^{j}\right)=\delta_{j}^{i}$. Considere $\eta^{1}, \eta^{2}$ duais a $X^{1}$, $X^{2}$; como são linearmente independentes, $\eta^{1} \wedge \eta^{2}$ é base de $\Omega^{2}(U)$, logo existem funções $a, b: U \rightarrow \mathbb{R}$ tais que

$$
\left\{\begin{array}{l}
d \eta^{1}=a \eta^{1} \wedge \eta^{2} \\
d \eta^{2}=b \eta^{1} \wedge \eta^{2} .
\end{array}\right.
$$

Defina $\alpha=-a \eta^{1}-b \eta^{2}$. Claramente $d \eta^{1}=-\alpha \wedge \eta^{2}$ e $d \eta^{2}=\alpha \wedge \eta^{1}$. Como $F^{*}\left(\omega^{i}\right)=\eta^{i}$, então

$$
\begin{aligned}
d \eta^{i} & =F^{*}\left(d \omega^{i}\right) \\
& =F^{*}\left(-\omega_{j}^{i} \wedge \omega^{j}\right) \\
& -F^{*}\left(\omega_{j}^{i}\right) \wedge \eta^{j} .
\end{aligned}
$$

Se $i=1$, então $d \eta^{1}=-F^{*}\left(\omega_{2}^{1}\right) \wedge \eta^{2}$ e para $i=2$ temos $d \eta^{2}=-F^{*}\left(\omega_{1}^{2}\right) \wedge \eta^{1}=F^{*}\left(\omega_{2}^{1}\right) \wedge \eta^{1}$, assim $\alpha=F^{*}\left(\omega_{2}^{1}\right)$. Se $\tilde{X}^{i}, i=1,2$, são outros campos $g$-ortonormais em $U$, sabemos que 
$\widetilde{\omega}=R^{-1} \omega R+R^{-1} d R$. Em coordenadas

$$
\begin{aligned}
\left(\begin{array}{cc}
0 & \widetilde{\omega}_{2}^{1} \\
-\widetilde{\omega}_{2}^{1} & 0
\end{array}\right) & =\left(\begin{array}{cc}
\cos \theta & -\sin \theta \\
\sin \theta & \cos \theta
\end{array}\right)\left(\begin{array}{cc}
0 & \omega_{2}^{1} \\
-\omega_{2}^{1} & 0
\end{array}\right)\left(\begin{array}{cc}
\cos \theta & \sin \theta \\
-\sin \theta & \cos \theta
\end{array}\right)+\left(\begin{array}{cc}
0 & d \theta \\
-d \theta & 0
\end{array}\right) \\
& =\left(\begin{array}{cc}
\sin \theta \omega_{2}^{1} & \cos \theta \omega_{2}^{1} \\
-\cos \theta \omega_{2}^{1} & \sin \theta \omega_{2}^{1}
\end{array}\right)\left(\begin{array}{cc}
\cos \theta & \sin \theta \\
-\sin \theta & \cos \theta
\end{array}\right)+\left(\begin{array}{cc}
0 & d \theta \\
-d \theta & 0
\end{array}\right) \\
& =\left(\begin{array}{cc}
0 & \omega_{2}^{1} \\
-\omega_{2}^{1} & 0
\end{array}\right)+\left(\begin{array}{cc}
0 & d \theta \\
-d \theta & 0
\end{array}\right),
\end{aligned}
$$

ou seja, $\widetilde{\omega}_{2}^{1}=\omega_{2}^{1}+d \theta$. Logo $d \widetilde{\omega}_{2}^{1}=d \omega_{2}^{1}$, fazendo o pull-back por $F$, obtemos $d \widetilde{\alpha}=d \alpha$, em que $\widetilde{\alpha}$ é a forma induzida por $\widetilde{X}^{i}$. Seja $k: U \rightarrow \mathbb{R}$ função definida por $d \alpha=k \eta^{1} \wedge \eta^{2}$; temos que $k$ não depende de $X^{i}$. De fato, sejam $\widetilde{e}^{1}=\cos \theta e^{1}-\sin \theta e^{2}$ e $\widetilde{e}^{2}=\sin \theta e^{1}+\cos \theta e^{2}$ e $\widetilde{\eta}^{i}$ duais de $\widetilde{e}^{i}$. Então $d \widetilde{\alpha}=d \alpha$ implica em $\widetilde{k} \widetilde{\eta}^{1} \wedge \widetilde{\eta}^{2}=k \eta^{1} \wedge \eta^{2}$, e avaliando em $\left(\widetilde{e}^{1}, \widetilde{e}^{2}\right)$,

$$
\begin{aligned}
\widetilde{k} & =k \eta^{1} \wedge \eta^{2}\left(\cos \theta e^{1}-\sin \theta e^{2}, \sin \theta e^{1}+\cos \theta e^{2}\right) \\
& =k(\cos \theta \sin \theta \underbrace{\eta^{1} \wedge \eta^{2}\left(e^{1}, e^{1}\right)}_{0}+\cos ^{2} \theta \underbrace{\eta^{1} \wedge \eta^{2}\left(e^{1}, e^{2}\right)}_{1} \\
& -\sin ^{2} \theta \underbrace{\eta^{1} \wedge \eta^{2}\left(e^{2}, e^{1}\right)}_{-1}-\sin \theta \cos \theta \underbrace{\eta^{1} \wedge \eta^{2}\left(e^{2}, e^{2}\right)}_{0}) \\
& =k\left(\cos ^{2} \theta+\sin ^{2} \theta\right) \\
& =k .
\end{aligned}
$$

Portanto $k$ depende apenas de $g$. Além disso,

$$
\begin{aligned}
k \eta^{1} \wedge \eta^{2} & =d \alpha \\
& =d F^{*}\left(\omega_{2}^{1}\right) \\
& =F^{*}\left(K \omega^{1} \wedge \omega^{2}\right) \\
& =(K \circ F) F^{*}\left(\omega^{1}\right) \wedge F^{*}\left(\omega^{2}\right) \\
& =(K \circ F) \eta^{1} \wedge \eta^{2}
\end{aligned}
$$

e segue que $F^{*}(K)=k$, portanto $K$ depende apenas de $I$.

\subsection{Geometria intrínseca}

Na seção anterior consideramos o subfibrado $\left.\mathcal{F}^{1} \subset A S O(n)\right|_{M}$ sobre uma superfície $M^{n}$ de $\mathbb{E}^{n}$ e nesse espaço a forma de Maurer-Cartan nos dava certos invariantes de $M$. Nesta seção vamos procurar invariantes intrínsecos de $M$ de uma variedade Riemanniana. 
Definição 1.4.1. Seja $M^{n}$ uma variedade, e considere o fibrado dos referenciais em $M$,

$$
\mathcal{F}_{G L}(M):=\left\{\left(p, f_{p}\right): f_{p} \in L\left(T_{p} M, \mathbb{R}^{n}\right) \text { bijetora }\right\}
$$

Em $\mathcal{F}_{G L}(M)$ definimos a forma tautológica $\eta \in \Omega^{1}\left(\mathcal{F}_{G L}(M)\right) \otimes \mathbb{R}^{n}$ :

$$
\begin{aligned}
\eta: \mathcal{F}_{G L}(M) \longrightarrow T^{*}\left(\mathcal{F}_{G L}(M)\right) \otimes \mathbb{R}^{n} & \\
f=\left(p, f_{p}\right) \longmapsto \eta_{f}: T_{f} \mathcal{F}_{G L}(M) & \longrightarrow \mathbb{R}^{n} \\
v & \longmapsto f_{p}\left(\pi_{*}(v)\right)
\end{aligned}
$$

em que $\pi: \mathcal{F}_{G L}(M) \rightarrow M$ é projeção do fibrado $\mathcal{F}_{G L}(M)$.

Observação 1.4.2. No contexto anterior, tome $\left(q^{1}, \cdots, q^{n}\right)$ coordenadas em torno de $p \in M$ e $\left(p^{11}, \cdots, p^{n n}\right)$ coordenadas em torno de algum $f_{p}$, e denote $f=\left(p, f_{p}\right)$. Então

$$
\begin{aligned}
\eta_{f}\left(v^{i} \frac{\partial}{\partial q^{i}}+v^{i j} \frac{\partial}{\partial p^{i j}}\right) & =\left(\begin{array}{ccc}
p^{11} & \cdots & p^{1 n} \\
\vdots & \ddots & \vdots \\
p^{n 1} & \cdots & p^{n n}
\end{array}\right)\left(\begin{array}{c}
v^{1} \\
\vdots \\
v^{n}
\end{array}\right) \\
& =\left(\begin{array}{c}
\sum_{i=1}^{n} p^{1 i} v^{i} \\
\vdots \\
\sum_{i=1}^{n} p^{n i} v^{i}
\end{array}\right),
\end{aligned}
$$

Portanto cada coordenada de $\eta_{f}$, denotada $\eta^{i}$, é localmente $\eta^{i}$ é igual a $\sum_{j=1}^{n} p^{i j} d q^{j}$.

O próximo passo é mostrar que as formas $\eta^{i}$ generalizam para $\mathcal{F}_{G L}(M)$ o que tínhamos em $A S O(n) \operatorname{com} \omega^{i}$, isto é, vamos mostrar a existência de formas $\alpha_{j}^{i}$ que, junto com $\eta^{i}$, formam um correferencial de $\mathcal{F}_{G L}(M)$ e satisfazem as equações

$$
d \eta^{i}=-\alpha_{j}^{i} \wedge \eta^{j}
$$

Considere $\left\{\widetilde{\eta}^{1}, \cdots, \widetilde{\eta}^{n}\right\}$ correferencial local em $U \subset M$, defina $\widetilde{\eta} \in \Omega^{1}(U) \otimes \mathbb{R}^{n}$ por $\widetilde{\eta}_{p}(v)=$ $\sum_{i=1}^{n} \widetilde{\eta}_{p}^{i}(v) e^{i}$, em que $e_{i}$ base canônica do $\mathbb{R}^{n}$ e seja $t$ a trivialização local

$$
\begin{aligned}
t: G L_{n}(\mathbb{R}) \times U & \left.\longrightarrow \mathcal{F}_{G L}(M)\right|_{U} \\
(g, p) \longmapsto t(g, p): T_{p} U & \longrightarrow \mathbb{R}^{n} \\
v & \longmapsto g^{-1} \widetilde{\eta}_{p}(v) .
\end{aligned}
$$


Observe que $\pi \circ t=\pi_{2}: G L_{n}(\mathbb{R}) \times U \rightarrow U$, projeção na segunda coordenada, assim

$$
\begin{aligned}
t^{*}(\eta)_{(g, p)}(A, v) & =\eta_{t(g, p)}\left(d t_{(g, p)(A, v)}\right) \\
& =t(g, p)\left(d \pi_{t(g, p)}\left(d t_{(g, p)}(A, v)\right)\right) \\
& =g^{-1} \widetilde{\eta}_{p}\left(d(\pi \circ t)_{(g, p)}(A, v)\right) \\
& =g^{-1} \widetilde{\eta}_{p}\left(d \pi_{2}\right)_{(g, p)}(A, v) \\
& =g^{-1} \widetilde{\eta}_{p}(v),
\end{aligned}
$$

ou seja,

$$
t^{*}(\eta) g^{-1} \widetilde{\eta}
$$

Note que para $\widetilde{\eta}^{i}$ existem $\widetilde{\alpha}_{j}^{i}$ satisfazendo $d \widetilde{\eta}^{i}=-\widetilde{\alpha}_{j}^{i} \wedge \widetilde{\eta}^{j}$, isto é, $d \widetilde{\eta}=-\widetilde{\alpha} \wedge \widetilde{\eta}$. De (1.9) temos

$$
\begin{aligned}
t^{*}(d \eta) & =d\left(g^{-1}\right) \wedge \widetilde{\eta}+g^{-1} d \widetilde{\eta} \\
& =-g^{-1} d g g^{-1} \wedge \widetilde{\eta}+g^{-1} d \widetilde{\eta} \\
& =-g^{-1} d g \wedge t^{*} \eta-g^{-1} d \widetilde{\eta} \\
& =-g^{-1} d g \wedge t^{*} \eta-g^{-1}(\widetilde{\alpha} \wedge \widetilde{\eta}) \\
& =-g^{-1} d g \wedge t^{*} \eta-\widetilde{\alpha} \wedge t^{*} \eta \\
& =-\left(g^{-1} d g+\widetilde{\alpha}\right) \wedge t^{*} \eta .
\end{aligned}
$$

Defina $\alpha_{j}^{i}=\left(t_{*}\left(g^{-1} d g+\widetilde{\alpha}\right)\right)_{j}^{i}$, da igualdade acima $d \eta=-\alpha \wedge \eta$. Como $\eta$ toma valores em $\mathbb{R}^{n}$, cada $\eta^{i}$ satisfaz $d \eta^{i}=-\alpha_{j}^{i} \wedge \eta^{j}$, o que verifica (1.8). Do fato que $\left(g^{-1} d g+\widetilde{\alpha}, \widetilde{\eta}\right)$ é correferencial em $G L_{n}(\mathbb{R}) \times U$ segue $(\eta, \alpha)$ correferencial de $\mathcal{F}_{G L}(M)$.

Proposição 1.4.3. Sejam $\beta_{j}^{i}$ formas tais que $(\eta, \beta)$ é correferencial de $\mathcal{F}_{G L}(M)$ e $\beta_{j}^{i}$ satisfazem (1.8), então existem $C_{j k}^{i}$, simétricos nos índices inferiores, com

$$
\beta_{k}^{i}=\alpha_{j}^{i}+C_{j k}^{i} \eta^{k}
$$

Reciprocamente, fixados $\alpha_{j}^{i}$ tais que $(\eta, \alpha)$ é correferencial de $\mathcal{F}_{G L}(M)$, e $\eta^{i}$ e $\alpha_{j}^{i}$ satisfazendo (1.8), então para quaisquer funções $C_{j k}^{i}$, simétricas nos índices inferiores, as formas $\beta_{j}^{i}=\alpha_{j}^{i}+C_{j k}^{i} \eta^{k}$ têm as mesmas propriedades.

Demonstração. Por hipótese, $\alpha_{j}^{i} \wedge \eta^{j}=\beta_{j}^{i} \wedge \eta^{j}$, isto é, $\left(\alpha_{j}^{i}-\beta_{j}^{i}\right) \wedge \eta^{j}=0$, com $\eta^{j}$ linearmente independentes. Do Lema de Cartan segue o afirmado. Para recíproca, basta verificar que 
$d \eta^{i}=\beta_{j}^{i} \wedge \eta^{j}$, uma vez que se $(\eta, \alpha)$ é correferencial, então $(\eta, \alpha+C \eta=\beta)$ também é correferencial. Desenvolvendo $\beta_{j}^{i} \wedge \eta^{j}$, obtemos

$$
\begin{aligned}
\beta_{j}^{i} \wedge \eta^{j} & =\left(\alpha_{j}^{i}+C_{j k}^{i} \eta^{k}\right) \wedge \eta^{j} \\
& =\alpha_{j}^{i} \wedge \eta^{j}+C_{j k}^{i} \eta^{k} \wedge \eta^{j} \\
& =d \eta^{i}+C_{j k}^{i} \eta^{k} \wedge \eta^{j} \\
& =d \eta^{i}+\sum_{j<k}(\underbrace{C_{j k}^{i}-C_{k j}^{i}}_{C_{j k}^{i}=C_{k j}^{i}}) \eta^{k} \wedge \eta^{j} \\
& =d \eta^{i} .
\end{aligned}
$$

Segue da proposição anterior que não vale a unicidade de $\alpha_{j}^{i}$. Entretanto, sendo $\left(M^{n}, g\right)$ uma variedade Riemanniana, o subfibrado $\mathcal{F}_{M}:=\left\{\left(p, f_{p}\right): f_{p} \in L\left(T_{p} M, \mathbb{E}^{n}\right)\right.$ isometria $\} \subset$ $\mathcal{F}_{G L}(M)$ dos referenciais ortonormais tem a seguinte propriedade: sejam $s, s(p)=\left(p, f_{p}\right)$, uma seção local de $\mathcal{F}_{M}$ e $\eta$ a forma tautológica em $\mathcal{F}_{M}$. Defina $\bar{\eta}=s^{*}(\eta)$. Então para $p$ no domínio de $s$ e $v, w \in T_{p} M$, temos

$$
\begin{aligned}
\left\langle\bar{\eta}_{p}(v), \bar{\eta}_{p}(w)\right\rangle & =\left\langle\eta_{s(p)}\left(s_{*}(v)\right), \eta_{s(p)}\left(s_{*}(w)\right)\right\rangle \\
& =\left\langle f_{p}\left(\pi_{*}\left(s_{*}(v)\right)\right), f_{p}\left(\pi_{*}\left(s_{*}(w)\right)\right)\right\rangle \\
& =\left\langle f_{p}(v), f_{p}(w)\right\rangle \\
& =g_{p}(v, w),
\end{aligned}
$$

em que a última igualdade segue de $f_{p}$ ser isometria. Por outro lado, $\bar{\eta}_{p}=\sum_{i=1}^{n} \bar{\eta}_{p}^{i} e^{i}$, então para cada $v, w \in T_{p} M$ vale $\left\langle\bar{\eta}_{p}(v), \bar{\eta}_{p}(w)\right\rangle=\sum_{i=1}^{n} \bar{\eta}_{p}^{i}(v) \bar{\eta}_{p}^{i}(w)$, portanto

$$
g=\sum_{i=1}^{n}\left(\bar{\eta}^{i}\right)^{2} .
$$

Com a estrutura adicional de $\mathcal{F}_{M}$, temos o seguinte resultado

Teorema 1.4.4. Sejam $\left(M^{n}, g\right)$ variedade Riemanniana e $\eta^{i}$ as formas tautológicas em $\mathcal{F}_{M}$. Fixada uma seção local s $: M \rightarrow \mathcal{F}_{M}$, existem e são únicas formas $\eta_{j}^{i} \in \Omega^{1}(M)$ tais que $s^{*}\left(d \eta^{i}\right)=-\eta_{j}^{i} \wedge s^{*}\left(\eta^{j}\right)$ e $\eta$ toma valores em $\mathfrak{s o}(n)$, isto é, $\eta_{j}^{i}=-\eta_{i}^{j}$. 
Demonstração. Considere $\bar{\eta}^{i}=s^{*}\left(\eta^{i}\right)$, vimos que existem, porém não necessariamente únicas, formas $\alpha_{j}^{i}$ tais que $d \bar{\eta}^{i}=-\alpha_{j}^{i} \wedge \bar{\eta}^{j}$, escrevemos

$$
\alpha_{j}^{i}=\underbrace{\frac{1}{2}\left(\alpha_{j}^{i}-\alpha_{i}^{j}\right)}_{\beta_{j}^{i}}+\underbrace{\frac{1}{2}\left(\alpha_{j}^{i}+\alpha_{i}^{j}\right)}_{\gamma_{j}^{i}},
$$

em que $\beta_{j}^{i}$ é anti-simétrica e $\gamma_{j}^{i}$ é simétrica. Primeiro provaremos a existência. Sejam $T_{j k}^{i}$ definido por $\gamma_{j}^{i}=T_{j k}^{i} \bar{\eta}^{k}$, note que $T_{j k}^{i}=T_{i k}^{j}$, e defina $\eta_{j}^{i}=\alpha_{j}^{i}-\left(T_{j k}^{i}+T_{k j}^{i}-T_{k i}^{j}\right) \bar{\eta}^{k}$. Então

$$
\begin{aligned}
-\eta_{j}^{i} \wedge \bar{\eta}^{j} & =-\left(\alpha_{j}^{i}-\left(T_{j k}^{i}+T_{k j}^{i}-T_{k i}^{j}\right) \bar{\eta}^{k}\right) \wedge \bar{\eta}^{j} \\
& =d \bar{\eta}^{i}+\left(T_{j k}^{i}+T_{k j}^{i}-T_{k i}^{j}\right) \bar{\eta}^{k} \wedge \bar{\eta}^{j} \\
& =d \bar{\eta}^{i}+\sum_{j<k}\left(\left(T_{j k}^{i}+T_{k j}^{i}-T_{k i}^{j}\right)-\left(T_{k j}^{i}+T_{j k}^{i}-T_{j i}^{k}\right)\right) \bar{\eta}^{k} \wedge \bar{\eta}^{j} \\
& =d \bar{\eta}^{i}+\sum_{j<k} \underbrace{\left(T_{j i}^{k}-T_{k i}^{j}\right)}_{\text {simetria }} \bar{\eta}^{k} \wedge \bar{\eta}^{i} \\
& =d \bar{\eta}^{k} .
\end{aligned}
$$

Observe que

$$
\begin{aligned}
\frac{1}{2}\left(\eta_{j}^{i}+\eta_{i}^{j}\right) & =\frac{1}{2}\left(\alpha_{j}^{i}-\left(T_{j k}^{i}+T_{k j}^{i}-T_{k i}^{j}\right) \bar{\eta}^{k}\right)+\frac{1}{2}\left(\alpha_{i}^{j}-\left(T_{i k}^{j}+T_{k i}^{j}-T_{k j}^{i}\right) \bar{\eta}^{k}\right) \\
& =\underbrace{\frac{1}{2}\left(\alpha_{j}^{i}+\alpha_{i}^{j}\right)}_{\text {def. } \gamma_{j}^{i}}-\frac{1}{2} \underbrace{\left(T_{j k}^{i}+T_{i k}^{j}\right)}_{\text {simetria }} \bar{\eta}^{k} \\
& =\gamma_{j}^{i}-T_{j k}^{i} \bar{\eta}^{k} \\
& =\gamma_{j}^{i}-\gamma_{j}^{i} \\
& =0
\end{aligned}
$$

portanto $\eta_{j}^{i}=-\eta_{i}^{j}$.

Resta provar a unicidade, suponha $\delta_{j}^{i}$ nas mesmas condições que $\eta_{j}^{i}$. Então $\left(\delta_{j}^{i}-\eta_{j}^{i}\right) \wedge$ $\bar{\eta}^{j}=0$. Pelo Lema de Cartan existem $C_{j k}^{i}$, simétricas nos índices inferiores, tais que $\delta_{j}^{i}-\eta_{j}^{i}=C_{j k}^{i} \bar{\eta}^{k}$. Como $\delta$ e $\eta$ são anti-simétricos, temos $C_{j k}^{i}=-C_{i k}^{j}$, portanto

$$
C_{j k}^{i}=-C_{i k}^{j}=-C_{k i}^{j}=C_{j i}^{k}=C_{i j}^{k}=-C_{k j}^{i}=-C_{j k}^{i},
$$

$\operatorname{logo} C_{j k}^{i} \equiv 0$ e segue a unicidade.

Observação 1.4.5. Suponha $M^{n}$ subvariedade Riemanniana de $\mathbb{E}^{n+s}$ e seja $F: M \rightarrow \mathcal{F}^{1}$ qualquer extensão de $s: M \rightarrow \mathcal{F}_{M}$. Temos que $F^{*}\left(\omega_{j}^{i}\right)$ satisfazem as equações $d \bar{\eta}^{i}=$ $-F^{*}\left(\omega_{j}^{i}\right) \wedge \bar{\eta}^{j}$ e a anti-simetria. Pela unicidade, $\eta_{j}^{i}=F^{*}\left(\omega_{j}^{i}\right)$. 
É verdade uma versão mais sofisticada do teorema acima,

Teorema 1.4.6. Sejam $\left(M^{n}, g\right)$ variedade Riemanniana e $\eta^{i}$ formas tautológicas em $\mathcal{F}_{M}$, então existem $\eta_{j}^{i} \in \Omega^{1}\left(\mathcal{F}_{M}\right)$ tais que $\left(\eta^{i}, \eta_{j}^{i}\right)$ é correferencial de $\mathcal{F}_{M}$

$$
d \eta^{i}=-\eta_{j}^{i} \wedge \eta^{j}
$$

e $\eta$ toma valores em $\mathfrak{s o}(\mathfrak{n})$, isto é, $\eta_{j}^{i}=-\eta_{i}^{j}$. As formas $\eta_{j}^{i}$ são ditas formas de conexão de $M$.

Demonstração. [Morita, 2001, p. 264].

Derivando (1.11) obtemos $0=d \eta_{j}^{i} \wedge \eta^{j}-\eta_{j}^{i} \wedge d \eta^{j}$, mas $d \eta^{j}=-\eta_{k}^{j} \wedge \eta^{k}$, então $0=$ $d \eta_{j}^{i} \wedge \eta^{j}+\eta_{j}^{i} \wedge \eta_{k}^{j} \wedge \eta^{k}=\left(d \eta_{j}^{i}+\eta_{k}^{i} \wedge \eta_{j}^{k}\right) \wedge \eta^{j}$, defina $\Theta_{j}^{i}:=d \eta_{j}^{i}+\eta_{k}^{i} \wedge \eta_{j}^{k}$, então

Proposição 1.4.7. $\Theta:=\Theta_{j}^{i} \otimes \eta^{j} \otimes e_{i} \in \Omega^{2}\left(\mathcal{F}_{M}, T^{*} M \otimes T M\right)$ é básica, em que e $e_{i}$ é dual $a \eta^{i}$.

Demonstração. Seja $(p, g) \in \mathcal{F}_{M}$, isto é, $g: T_{p} M \rightarrow \mathbb{E}^{n}$ uma isometria. Identificando as isometrias com $O(n)$, outro elemento na mesma fibra é da forma $(p, g R)$, em que $R \in O(n)$. Escreva $\widetilde{\Theta}=d \eta+\eta \wedge \eta \in \Omega^{2}\left(\mathcal{F}_{M}\right) \otimes M_{n}(\mathbb{R})$, ou seja $\widetilde{\Theta}$ é matriz com coeficientes $\Theta_{j}^{i}$. Novamente usando a trivialização $t: O(n) \times U \rightarrow \mathcal{F}_{M}$, temos

$$
\begin{aligned}
t^{*} \eta_{(p, g R)} & =(g R)^{-1} d(g R)+(g R)^{-1} \bar{\eta}(g R) \\
& =R^{-1}\left(g^{-1} d g+g^{-1} \bar{\eta} g\right) R+R^{-1} d R \\
& =R^{-1} t^{*} \eta_{(p, g)} R+R^{-1} d R,
\end{aligned}
$$

então

$d t^{*} \eta_{(p, g R)}=-R^{-1} d R R^{-1} \wedge t^{*} \eta_{(p, g)} R+R^{-1} d t^{*} \eta_{(p, g)} R-R^{-1} t^{*} \eta_{(p, g)} \wedge d R-R^{-1} d R R^{-1} \wedge d R$ Explicitando $t^{*} \eta_{(p, g R)} \wedge t^{*} \eta_{(p, g R)}$

$$
\begin{aligned}
t^{*}(\eta)_{(p, g R)} \wedge t^{*}(\eta)_{(p, g R)} & =\left(R^{-1} t^{*} \eta_{(p, g)} R+R^{-1} d R\right) \wedge\left(R^{-1} t^{*} \eta_{(p, g)} R+R^{-1} d R\right) \\
& =R^{-1} t^{*} \eta_{(p, g)} \wedge t^{*} \eta_{(p, g)} R+R^{-1} t^{*} \eta_{(p, g)} \wedge d R \\
& +R^{-1} d R R^{-1} \wedge t^{*} \eta_{(p, g)} R+R^{-1} d R \wedge R^{-1} d R
\end{aligned}
$$


somando $d t^{*} \eta_{(p, g R)}+t^{*} \eta_{(p, g R)} \wedge t^{*} \eta_{(p, g R)}$, temos

$$
\begin{aligned}
t^{*} \widetilde{\Theta}_{(p, g R)} & =d t^{*} \eta_{(p, g R)}+t^{*} \eta_{(p, g R)} \wedge t^{*} \eta_{(p, g R)} \\
& =R^{-1}\left(d t^{*} \eta_{(p, g)}+t^{*} \eta_{(p, g)} \wedge t^{*} \eta_{(p, g)}\right) R \\
& =R^{-1} t^{*} \widetilde{\Theta}_{(p, g)} R,
\end{aligned}
$$

usando o fato que $R^{-1}=R^{t}$, em cada coeficiente $\left.\Theta_{j}^{i}\right|_{(p, g R)}=\left.R_{k}^{i} \Theta_{l}^{k}\right|_{(p, g)} R_{j}^{l}$, analogamente $\eta_{(p, g R)}^{j}=R_{s}^{j} \eta_{(p, g)}^{s}$ e $\left.e_{i}\right|_{(p, g R)}=\left.R_{i}^{t} e_{t}\right|_{(p, g)}, \log \mathrm{O}$

$$
\begin{aligned}
\Theta_{(p, g R)} & =\left.\left.\Theta_{j}^{i}\right|_{(p, g R)} \otimes \eta_{(p, g R)}^{j} \otimes e_{i}\right|_{(p, g R)} \\
& =\left.\left.R_{k}^{i} \Theta_{l}^{k}\right|_{(p, g)} R_{j}^{l} \otimes R_{s}^{j} \eta_{(p, g)}^{s} \otimes R_{i}^{t} e_{t}\right|_{(p, g)} \\
& =\left.\left.R_{k}^{i} R_{i}^{t} \Theta_{l}^{k}\right|_{(p, g)} R_{j}^{l} R_{s}^{j} \otimes \eta_{(p, g)}^{s} \otimes e_{t}\right|_{(p, g)} \\
& =\left.\left.\delta_{t}^{k} \delta_{s}^{l} \Theta_{l}^{k}\right|_{(p, g)} \otimes \eta_{(p, g)}^{s} \otimes e_{t}\right|_{(p, g)} \\
& =\left.\left.\Theta_{s}^{t}\right|_{(p, g)} \otimes \eta_{(p, g)}^{s} \otimes e_{t}\right|_{(p, g)} \\
& =\Theta_{(p, g)},
\end{aligned}
$$

isto é, $\Theta$ é constante nas fibras de $\mathcal{F}_{M}$, logo básica.

Definição 1.4.8. Para qualquer seção local $s: M \rightarrow \mathcal{F}_{M}$ defina $\Omega:=s^{*}(\Theta)$. Como $\Theta$ é básica, $\Omega \in \Omega^{1}\left(M, T^{*} M \otimes T M\right)$ está bem definida. Dizemos que $\Omega$ é o forma de curvatura de $M$.

Observação 1.4.9. Note que $\Omega$ toma valores em $T^{*} M \otimes T M \cong \operatorname{End}(T M)$. Quando fixado um referencial, podemos identifica-lo com um espaço de matrizes. Como $\Theta_{j}^{i}=-\Theta_{i}^{j}$, segue da definição de $\Omega$ que $\Omega_{j}^{i}=-\Omega_{i}^{j}$, isto é, $\Omega \in \Omega^{2}(M, \mathfrak{s o}(T M))$.

Definição 1.4.10. Uma variedade Riemanniana $\left(M^{n}, g\right)$ é dita flat se para cada ponto existe um sistema de coordenadas $\left(x^{1}, \cdots, x^{n}\right)$ em torno desse ponto tal que $g=\left(d x^{1}\right)^{2}+$ $\cdots+\left(d x^{n}\right)^{2}$.

Teorema 1.4.11. Seja $\left(M^{n}, g\right)$ uma variedade Riemanniana cuja forma de curvatura $\Omega \equiv 0$, então $M$ é flat.

Demonstração. Por hipótese, $d \eta=\Omega-\eta \wedge \eta=-\eta \wedge \eta$. Aplicando o Teorema 1.2.11 para $G=S O(n)$, em torno de cada $p \in M$ existe $g: U \rightarrow S O(n)$ tal que $\eta_{j}^{i}=\left(g^{-1} d g\right)_{j}^{i}$, logo 
$d \eta^{j}=-\left(g^{-1} d g\right)_{k}^{j} \wedge \eta^{k}$. Defina $\bar{\eta}^{i}=g_{j}^{i} \eta^{j}$. Então

$$
\begin{aligned}
d \bar{\eta}^{i} & =d g_{j}^{i} \wedge \eta^{j}+g_{j}^{i} d \eta^{j} \\
& =d g_{j}^{i} \wedge \eta^{j}+g_{j}^{i}\left(-\left(g^{-1} d g\right)_{k}^{j} \wedge \eta^{k}\right) \\
& =d g_{j}^{i} \wedge \eta^{j}-d g_{k}^{i} \wedge \eta^{k} \\
& =0 .
\end{aligned}
$$

De (1.10) temos $g=\sum_{i=1}^{n}\left(\eta^{i}\right)^{2}$. Como $\bar{\eta}=g \eta$ e $g \in O(n)$, segue que $\sum_{i=1}^{n}\left(\eta^{i}\right)^{2}=\sum_{i=1}^{n}\left(\bar{\eta}^{i}\right)^{2}$. Do fato que $d \bar{\eta}=0$, existem funções localmente definidas $\left(x^{i}, \cdots, x^{n}\right)$ tais que $d x^{i}=\bar{\eta}^{i}$, logo $g=\sum_{i=1}^{n}\left(d x^{i}\right)^{2}$, portanto $M$ é flat.

Observação 1.4.12. $\Omega_{j}^{i} \in \Omega^{2}(M)$, então existem funções $R_{j k l}^{i}$ tais que $\Omega_{j}^{i}=\frac{1}{2} R_{j k l}^{i} \eta^{k} \wedge$ $\eta^{l}=\sum_{k<l} R_{j k l}^{i} \eta^{k} \wedge \eta^{l}$, e verifica-se imediatamente que $R_{j k l}^{i}$ é anti-simétrica em $i j$ e $k l$. De (1.11) e da definição de $\Omega$,

$$
\left\{\begin{array}{l}
d \eta^{i}=-\eta_{j}^{i} \wedge \eta^{j} \\
d \eta_{j}^{i}=-\eta_{k}^{i} \wedge \eta_{j}^{k}+\frac{1}{2} R_{j k l}^{i} \eta^{k} \wedge \eta^{l} .
\end{array}\right.
$$

As equações acima são ditas equações de estrutura de $M$.

Observação 1.4.13. No Teorema 1.4.6, definimos as formas de conexão $\eta_{j}^{i}$. No que segue vamos justificar esse nome.

Sejam $X \in \Gamma(T M)$ e $s: M \rightarrow \mathcal{F}_{M}$ uma seção local. Definimos a conexão de $M$ como

$$
\nabla X=\left(d X^{i}+X^{j} \eta_{j}^{i}\right) \otimes e_{i}
$$

em que $e_{i}$ é referencial ortonormal dado por $s$ e $X=X^{i} e_{i}$.

Afirmação: $\nabla X$ não depende de $s$. Seja $\bar{s}: M \rightarrow \mathcal{F}_{M}$ uma outra seção. Então existe uma função $R: M \rightarrow O(n)$ tal que

$$
\left\{\begin{array}{l}
\bar{e}_{i}=R_{i}^{u} e_{u} \\
\bar{X}^{j}=\left(R^{-1}\right)_{k}^{j} X^{k} \\
\bar{\eta}_{j}^{i}=\left(R^{-1}\right) i_{s} \eta_{t}^{s} R_{j}^{t}+\left(R^{-1}\right)_{v}^{i} d R_{j}^{v} \\
d\left(R^{-1}\right)_{l}^{i}=-\left(R^{-1}\right)_{w}^{i} d R_{z}^{w}\left(R^{-1}\right)_{l}^{z}
\end{array}\right.
$$


Assim

$$
\begin{aligned}
\nabla \bar{X} & =\left(d \bar{X}^{i}+\bar{X}^{j} \bar{\eta}_{j}^{i}\right) \otimes \bar{e}_{i} \\
& =\left(d\left(\left(R^{-1}\right)_{l}^{i} X^{l}\right)+\left(R^{-1}\right)_{k}^{j} X^{k}\left(\left(R^{-1}\right)_{s}^{i} \eta_{t}^{s} R_{j}^{t}+\left(R^{-1}\right)_{v}^{i} d R_{j}^{v}\right)\right) \otimes R_{i}^{u} e_{u} \\
& =d\left(R^{-1}\right)_{l}^{i} R_{i}^{u} X^{l} \otimes e_{u}+\left(R^{-1}\right)_{l}^{i} R_{i}^{u} d X^{l} \otimes e_{u} \\
& +\left(R^{-1}\right)_{k}^{j}\left(R^{-1}\right)_{s}^{i} R_{j}^{t} R_{i}^{u} X^{k} \eta_{t}^{s} \otimes e_{u}+\left(R^{-1}\right)_{k}^{j}\left(R^{-1}\right)_{v}^{i} R_{i}^{u} X^{k} d R_{j}^{v} \otimes e_{u} \\
& =-\underbrace{\left(R^{-1}\right)_{w}^{i} R_{i}^{u}}_{\delta_{w}^{u}}\left(R^{-1}\right)_{l}^{z} X^{l} d R_{z}^{w} \otimes e_{u}+\underbrace{\left(R^{-1}\right)_{l}^{i} R_{i}^{u}}_{\delta_{l}^{u}} d X^{l} \otimes e_{u} \\
& +\underbrace{\left(R^{-1}\right)_{k}^{j}\left(R^{-1}\right)_{s}^{i} R_{j}^{t} R_{i}^{u}}_{\delta_{k}^{t} \delta_{s}^{u}} X^{k} \eta_{t}^{s} \otimes e_{u}+\left(R^{-1}\right)_{k}^{j} \underbrace{\left(R^{-1}\right)_{v}^{i} R_{i}^{u}}_{\delta_{v}^{u}} X^{k} d R_{j}^{v} \otimes e_{u} \\
& =\underbrace{-\left(R^{-1}\right)_{l}^{z} X^{l} d R_{z}^{u} \otimes e_{u}+\left(R^{-1}\right)_{k}^{j} X^{k} d R_{j}^{u} \otimes e_{u}}+d X^{u} \otimes e_{u}+X^{k} \eta_{k}^{u} \otimes e_{u} \\
& =\left(d X^{u}+X^{k} \eta_{k}^{u}\right) \otimes e_{u} \\
& =\nabla X,
\end{aligned}
$$

portanto $\nabla X$ está bem definida e definida em toda $M$. Defina $\left.\nabla_{Y} Z:=Y\right\lrcorner \nabla X \in \Gamma(T M)$, então $\nabla_{Y}(f X)=f \nabla_{Y} X+Y(f) X ;$ de fato,

$$
\begin{aligned}
\nabla_{Y} f X & =\left(d\left(f X^{i}\right)(Y)+f X^{j} \eta_{j}^{i}(Y)\right) e_{i} \\
& =d f(Y) X^{i} e_{i}+\left(f d X^{i}(Y)+f X^{j} \eta_{j}^{i}(Y)\right) e_{i} \\
& =Y(f) X+f \nabla_{Y} X
\end{aligned}
$$

A aplicação $\nabla: \Gamma(T M)^{2} \rightarrow \Gamma(T M)$ também satisfaz $\nabla_{Y} X-\nabla_{X} Y=[X, Y]$, para cada $X, Y \in \Gamma(T M)$. Observe que se $\left(x^{i}, \cdots, x^{n}\right)$ são coordenadas locais e $\left\{\partial_{1}, \cdots, \partial_{n}\right\}$ o referencial dado pelas coordenadas, temos $\nabla_{\partial_{i}} \partial_{j}-\nabla_{\partial_{j}} \partial_{i}=\left[\partial_{i}, \partial_{j}\right]=0$. Devido a esse fato, dizemos que $\nabla$ é simétrica. A simetria de $\nabla$ deve-se essencialmente ao seguinte resultado

Proposição 1.4.14. Sejam $\omega \in \Omega^{1}(M)$ e $X, Y \in \Gamma(T M)$. Então

$$
d \omega(X, Y)=X(\omega(Y))-Y(\omega(X))-\omega([X, Y])
$$

Demonstração. [Lee, 2012, p. 369]

Verificando a simetria. Seja $s: M \rightarrow \mathcal{F}_{M}$. Dados $X, Y \in \Gamma(T M)$, escreva $X=X^{i} e_{i}$ e $Y=Y^{j} e_{j}$. Então $\nabla_{X} Y-\nabla_{Y} X=d Y^{i}(X) e_{i}-d X^{i}(Y) e_{i}+\sum_{i, j=1}^{n} X^{i} Y^{j}\left(\nabla_{e_{i}} e_{j}-\nabla_{e_{j}} e_{i}\right)$, então 
basta verificar a propriedade para $e_{i}$ e $e_{j}$. Por definição, $\nabla_{e_{i}} e_{j}=\eta_{j}^{s}\left(e_{i}\right) e_{s}, \operatorname{logo}$

$$
\nabla_{e_{i}} e_{j}-\nabla_{e_{j}} e_{i}=\left(\eta_{j}^{s}\left(e_{i}\right)-\eta_{i}^{s}\left(e_{j}\right)\right) e_{s}
$$

Usando a primeira equação (1.12), e (1.13) para $\eta^{s}, e_{i}$ e $e_{j}$,

$$
\begin{gathered}
d \eta^{s}\left(e_{i}, e_{j}\right)=-\eta_{k}^{s} \wedge \eta^{k}\left(e_{i}, e_{j}\right) \\
=-\eta_{j}^{s}\left(e_{i}\right)+\eta_{i}^{s}\left(e_{j}\right), \\
d \eta^{s}\left(e_{i}, e_{j}\right)=e_{i}\left(\eta^{s}\left(e_{j}\right)\right)-e_{j}\left(\eta^{s}\left(e_{i}\right)\right)-\eta^{s}\left(\left[e_{i}, e_{j}\right]\right) \\
=\underbrace{e_{i}\left(\delta_{j}^{s}\right)}_{0}-\underbrace{e_{j}\left(\delta_{i}^{s}\right)}_{0}-\eta^{s}\left(\left[e_{i}, e_{j}\right]\right) \\
=-\eta^{s}\left(\left[e_{i}, e_{j}\right]\right),
\end{gathered}
$$

ou seja $\eta^{s}\left(\left[e_{i}, e_{j}\right]\right)=\eta_{j}^{s}\left(e_{i}\right)-\eta_{i}^{s}\left(e_{j}\right)$, e substituindo em (1.14) obtemos

$$
\nabla_{e_{i}} e_{j}-\nabla_{e_{j}} e_{i}=\eta^{s}\left(\left[e_{i}, e_{j}\right]\right) e_{s}=\left[e_{i}, e_{j}\right]
$$

Podemos relacionar $\nabla$ com $g$ da seguinte forma: para cada $X, Y, Z \in \Gamma(T M)$ vale

$$
Z(g(X, Y))=g\left(\nabla_{Z} X, Y\right)+g\left(X, \nabla_{Z} Y\right)
$$

De fato, por linearidade podemos supor $Z=e_{k}$, e temos

$$
\begin{aligned}
& g\left(\nabla_{e_{k}} X, Y\right)+g\left(X, \nabla_{e_{k}} Y\right)=g\left(\left(d X^{i}\left(e_{k}\right)+X^{t} \eta_{t}^{i}\left(e_{k}\right)\right) e_{i}, Y^{j} e_{j}\right) \\
& +g\left(X^{s} e_{s},\left(d Y^{u}\left(e_{k}\right)+Y^{v} \eta_{v}^{u}\left(e_{k}\right)\right) e_{u}\right) \\
& =\sum_{i=1}^{n} d X^{i}\left(e_{k}\right) Y^{i}+X^{t} Y^{i} \eta_{t}^{i}\left(e_{k}\right) \\
& +\sum_{s=1}^{n} X^{s} d Y^{s}\left(e_{k}\right)+X^{s} Y^{v} \eta_{v}^{s}\left(e_{k}\right) \\
& =\underbrace{\sum_{i=1}^{n}\left(Y^{i} d X^{i}\left(e_{k}\right)+X^{i} d Y^{i}\left(e_{k}\right)\right)}_{e_{k}(g(X, Y))}+\sum_{i=1}^{n}\left(X^{t} Y^{i}+X^{i} Y^{t}\right) \eta_{t}^{i}\left(e_{k}\right) .
\end{aligned}
$$

Basta agora mostrar que $\sum_{i=1}^{n}\left(X^{t} Y^{i}+X^{i} Y^{t}\right) \eta_{t}^{i}\left(e_{k}\right)=\sum_{i=1}^{n} \sum_{t=1}^{n}\left(X^{t} Y^{i}+X^{i} Y^{t}\right) \eta_{t}^{i}\left(e_{k}\right)=0$. Para 
$i=t$ temos $\eta_{i}^{i} \equiv 0$, assim

$$
\begin{aligned}
\sum_{i=1}^{n} \sum_{t=1}^{n}\left(X^{t} Y^{i}+X^{i} Y^{t}\right) \eta_{t}^{i}\left(e_{k}\right) & =\sum_{i<t}\left(X^{t} Y^{i}+X^{i} Y^{t}\right) \eta_{t}^{i}\left(e_{k}\right)+\sum_{i>t}\left(X^{t} Y^{i}+X^{i} Y^{t}\right) \eta_{t}^{i}\left(e_{k}\right) \\
& =\sum_{i<t}\left(X^{t} Y^{i}+X^{i} Y^{t}\right) \eta_{t}^{i}\left(e_{k}\right)-\underbrace{\sum_{i>t}\left(X^{t} Y^{i}+X^{i} Y^{t}\right) \eta_{i}^{t}\left(e_{k}\right)}_{\text {trocando } i \leftrightarrow t} \\
& =\sum_{i<t}\left(X^{t} Y^{i}+X^{i} Y^{t}\right) \eta_{t}^{i}\left(e_{k}\right)-\sum_{i<t}\left(X^{i} Y^{t}+X^{t} Y^{i}\right) \eta_{t}^{i}\left(e_{k}\right) \\
& =0,
\end{aligned}
$$

como desejado.

A conexão acima é dita conexão de Levi-Civita. Um resultado clássico é a unicidade dessa conexão segundo as propriedades apresentas. A relação de $\Omega$ com a curvatura clássica é também imediata.

Definição 1.4.15. Para cada $X, Y, Z \in \Gamma(T M)$ defina

$$
R(X, Y) Z=\nabla_{X} \nabla_{Y} Z-\nabla_{Y} \nabla_{X} Z-\nabla_{[X, Y]} Z
$$

Proposição 1.4.16. $R$ é o tensor curvatura de $M$.

Demonstração. Sejam $s: M \rightarrow \mathcal{F}_{M}$ e $\left\{e_{1}, \cdots, e_{n}\right\}$ referencial ortonormal dado por $s$. Para cada $X, Y \in \Gamma(T M)$, escreva $R(X, Y) e_{i}=\bar{R}_{i}^{j}(X, Y) e_{j}$, considere a forma, com valores em $M_{n}(\mathbb{R})$, determinada por $R$

$$
\bar{R}=\bar{R}_{j}^{i} \otimes \eta^{i} \otimes e_{j}
$$

De [Morita, 2001, p. 188-189], temos $\bar{R} \in \mathfrak{s o}(T M)$ e também $d \eta=-\eta \wedge \eta+\bar{R}$, cuja demonstração é uma aplicação simples da Proposição 1.4.14. Da segunda equação de (1.12) temos

$$
\begin{aligned}
d \eta & =-\eta \wedge \eta+\Omega \\
& =-\eta \wedge \eta+\bar{R},
\end{aligned}
$$

portanto $\Omega=\bar{R}$.

Observação 1.4.17. Para $\left(M^{2}, g\right)$ temos $R_{212}^{1}=K$. De fato, vimos que $d \omega_{2}^{1}=K \omega^{1} \wedge \omega^{2}$, e da definição de $\Omega$ temos $d \omega_{2}^{1}=-\underbrace{\omega_{1}^{1} \wedge \omega_{2}^{1}}_{0}-\underbrace{\omega_{2}^{1} \wedge \omega_{2}^{2}}_{0}+\Omega_{2}^{1}$, como $\Omega_{2}^{1}=\bar{R}_{212}^{1} \omega^{1} \wedge \omega^{2}$, segue $K=\bar{R}_{212}^{1}$ 


\section{Fórmula de Gauss-Bonnet-Chern}

\subsection{Preliminares}

Neste capítulo estabeleceremos a celébre fórmula de Gauss-Bonnet-Chern para variedades fechadas e orientadas de dimensão par. Vamos nos guiar pela demonstração original desse fato, contida em [Chern, 1944]. No que segue, $(M, g)$ é uma variedade Riemanniana, $\operatorname{dim}(M)=n, \mathcal{F}_{M}$ é fibrado de referenciais de $M$ e $S M$ é o fibrado unitário de $M$.

Sejam $s: M \rightarrow \mathcal{F}_{M}$ seção local. Mantendo a notação do artigo original, denote (1.12) por

$$
\left\{\begin{array}{l}
d \omega^{i}=-\omega_{j}^{i} \wedge \omega^{j} \\
d \omega_{j}^{i}=-\omega_{k}^{i} \wedge \omega_{j}^{k}+\Omega_{j}^{i}
\end{array}\right.
$$

Escreva $s(p)=\left(x, e_{1}(p), \cdots, e_{n}(p)\right)$ e considere outra seção local, cuja domínio tem intersecção não vazia com o domínio de $s, t: M \rightarrow \mathcal{F}_{M}, t(x)=\left(p, f_{1}(p), \cdots, f_{n}(p)\right)$. Existem funções $a_{j}^{i}: M \rightarrow \mathbb{R}$ tais que $e_{i}=\sum_{j=1}^{n} a_{j}^{i} f_{j}$, em que $\left(a_{j}^{i}\right)$ é matriz ortogonal, por [Morita, 2001, p. 190], $\left(\Omega_{j}^{i}\right)_{t}=a_{k}^{i}\left(\Omega_{l}^{k}\right)_{s} a_{j}^{l}$.

Seja $n \in \mathbb{N}$ par. Pf $: M_{n}\left(\Omega^{*}(M)\right)=\Omega^{*}(M) \otimes M_{n}(\mathbb{R}) \longrightarrow \Omega^{*}(M)$, é definida por

$$
P f(A)=\frac{1}{2^{\frac{n}{2}}\left(\frac{n}{2}\right) !} \sum_{\sigma \in S_{n}} \operatorname{sgn}(\sigma) A_{\sigma(1) \sigma(2)} \wedge \cdots \wedge A_{\sigma(n-1) \sigma(n)} .
$$

Pf satisfaz

1. Se $A \in M_{n}\left(\Omega^{*}(M)\right)$ é anti-simétrica, então $\operatorname{Pf}(A)^{2}=\operatorname{det}(A)$ e

2. Se $A \in M_{n}\left(\Omega^{*}(M)\right)$ é anti-simétrica e $B \in M_{n}\left(\Omega^{*}(M)\right)$ é qualquer, então $P f(B \wedge$ $\left.A \wedge B^{T}\right)=\operatorname{Pf}(A)$.

Do capítulo anterior temos $\Omega_{j}^{i}=-\Omega_{i}^{j}$. Defina $\Omega=\left(\frac{-1}{2 \pi}\right)^{\frac{n}{2}} \operatorname{Pf}\left(\Omega_{j}^{i}\right)$. Da segunda propriedade de $\operatorname{Pf}$ segue que $\Omega$ está definida em toda $M$. Observe que $\operatorname{deg}\left(\Omega_{j}^{i}\right)=2$ implica 
$\operatorname{deg}(\Omega)=n$. Logo se $M$ é fechada e orientável, então $\int_{M} \Omega$ é um número que a priori depende da métrica $g$.

Definição 2.1.1. Seja $M$ variedade Riemanniana fechada e orientada, considere o $p$-ésimo espaço de cohomologia de De Rham $H_{d R}^{p}(M)$. Definimos a característica de Euler de $M$ por

$$
\chi(M)=\sum_{p=0}^{n}(-1)^{p} \operatorname{dim}\left(H_{d R}^{p}(M)\right) .
$$

Observação 2.1.2. O Teorema de De Rham [Lee, 2012, p. 484] garante que $\chi(M)$ é um invariante topológico, e o Teorema da Dualidade de Poincaré [Morita, 2001, p. 163] diz que $H_{d R}^{p}(M) \cong H_{d R}^{n-p}(M)$. Portanto, se $n$ é impar,

$$
\begin{aligned}
\chi(M) & =\sum_{p=0}^{\frac{n-1}{2}}(-1)^{p} \operatorname{dim}\left(H_{d R}^{p}(M)\right)+\sum_{p=\frac{n-1}{2}+1}^{n}(-1)^{p} \operatorname{dim}\left(H_{d R}^{p}(M)\right) \\
& =\sum_{p=0}^{\frac{n-1}{2}}(-1)^{p} \operatorname{dim}\left(H_{d R}^{p}(M)\right)+\sum_{q=0}^{\frac{n-1}{2}}(-1)^{q+\frac{n-1}{2}+1} \operatorname{dim}\left(H_{d R}^{q+\frac{n-1}{2}+1}(M)\right) \\
& =\sum_{p=0}^{\frac{n-1}{2}}(-1)^{p} \operatorname{dim}\left(H_{d R}^{p}(M)\right)+\overbrace{q=0}^{\frac{n-1}{2}}-(-1)^{\frac{n-1}{2}-q} \operatorname{dim}\left(H_{d R}^{n-q-\frac{n-1}{2}-1}(M)\right) \\
& =\sum_{p=0}^{\frac{n-1}{2}}(-1)^{p} \operatorname{dim}\left(H_{d R}^{p}(M)\right)-\overbrace{q=0}^{\frac{n-1}{2}}(-1)^{\frac{n-1}{2}-q} \operatorname{dim}\left(H_{d R}^{\frac{n-1}{2}-q}(M)\right) \\
& =\sum_{p=0}^{\frac{n-1}{2}}(-1)^{p} \operatorname{dim}\left(H_{d R}^{p}(M)\right)-\overbrace{p=0}^{\frac{n-1}{2}}(-1)^{p} \operatorname{dim}\left(H_{d R}^{p}(M)\right) \\
& =0 .
\end{aligned}
$$

Será fundamental para estabelecermos a fórmula de Guass-Bonnet-Chern o seguinte teorema.

Teorema 2.1.3. (Índice de Poincaré-Hopf). Seja $V \in \Gamma(M)$ com um númuero finitos zeros, digamos $\left\{x_{1}, \cdots, x_{s}\right\}$. Então

$$
\chi(M)=\sum_{i=1}^{s} i n d_{V}\left(x_{i}\right) .
$$

Observação 2.1.4. Vide [Guillemin, 1974].

A relação entre $\int_{M} \Omega$ e o invariante apresentado acima é conhecida como fórmula de Gauss-Bonnet-Chern, cujo enunciado é: 
Teorema 2.1.5. (Gauss-Bonnet-Chern). Seja $(M, g)$ uma variedade Riemanniana fechada, orientada e de dimensão par. Então

$$
\int_{M} \Omega=\chi(M)
$$

\subsection{Transgressão de $\Omega$}

O objetivo dessa seção é apresentar a ideia, devida a S.-S. Chern [Chern, 1944], de que $\pi^{*}(\Omega)$ é sempre exata no fibrado unitário $\pi: S M \rightarrow M$.

Vamos primeiro estabelecer algumas relações que serão usadas posteriormente.

Derivando primeira a equação de (2.1),

$$
\begin{aligned}
0 & =d\left(d \omega^{i}\right) \\
& =-d \omega_{j}^{i} \wedge \omega^{j}+\omega_{j}^{i} \wedge d \omega^{j} \\
& =-\left(-\omega_{k}^{i} \wedge \omega_{j}^{k}+\Omega_{j}^{i}\right) \wedge \omega^{j}+\omega_{j}^{i} \wedge\left(-\omega_{k}^{j} \wedge \omega^{k}\right) \\
& =\underbrace{\omega_{k}^{i} \wedge \omega_{j}^{k} \wedge \omega^{j}-\omega_{j}^{i} \wedge \omega_{k}^{j} \wedge \omega^{k}}_{0}-\Omega_{j}^{i} \wedge \omega^{j} \\
& =-\Omega_{j}^{i} \wedge \omega^{j}
\end{aligned}
$$

isto é,

$$
\Omega_{j}^{i} \wedge \omega^{j}=0
$$

Analogamente para a segunda equação de (2.1),

$$
\begin{aligned}
0 & =-d \omega_{k}^{i} \wedge \omega_{j}^{k}+\omega_{k}^{i} \wedge d \omega_{j}^{k}+d \Omega_{j}^{i} \\
& =-\left(-\omega_{l}^{i} \wedge \omega_{k}^{l}+\Omega_{k}^{i}\right) \wedge \omega_{j}^{k}+\omega_{k}^{i} \wedge\left(-\omega_{l}^{k} \wedge \omega_{j}^{l}+\Omega_{j}^{k}\right)+d \Omega_{j}^{i} \\
& =\underbrace{\omega_{l}^{i} \wedge \omega_{k}^{l} \wedge \omega_{j}^{k}-\omega_{k}^{i} \wedge \omega_{l}^{k} \wedge \omega_{j}^{l}}_{0}+\Omega_{k}^{i} \wedge \omega_{j}^{k}+\omega_{k}^{i} \wedge \Omega_{j}^{k}+d \Omega_{j}^{i} \\
& =\Omega_{k}^{i} \wedge \omega_{j}^{k}+\omega_{k}^{i} \wedge \Omega_{j}^{k}+d \Omega_{j}^{i}
\end{aligned}
$$

ou seja,

$$
\Omega_{k}^{i} \wedge \omega_{j}^{k}+\omega_{k}^{i} \wedge \Omega_{j}^{k}+d \Omega_{j}^{i}=0
$$

Sejam $x \in M$, uma $U$ vizinhança de $x$, uma $V$ seção local de $S M$ e uma seção local de $\mathcal{F}_{M}$ ambas definidas em $U$. Podemos escrever $V$ no referencial $\left\{e_{1}, \cdots, e_{n}\right\}$ dado por $s$, ou seja, $V=v^{i} e_{i}$. Como

$$
g(V, V)=1
$$


segue $\sum_{i=1}^{n} v^{i} v^{i}=1$, como vimos na definição de conexão, $\nabla V=\theta^{i} \otimes e_{i}$, em que $\theta^{i}=$ $d v^{i}+v^{j} \omega_{j}^{i}$. Derivando (2.4)

$$
\begin{aligned}
0 & =g\left(\nabla_{X} V, V\right)+g\left(V, \nabla_{X} V\right) \\
& =2 g\left(\theta^{i}(X) e_{i}, v^{j} e_{j}\right) \\
& =2 \sum_{i=1}^{n} \theta^{i}(X) v^{i}
\end{aligned}
$$

para todo $X \in \Gamma(M)$. Portanto

$$
\sum_{i=1}^{n} \theta^{i} v^{i}=0
$$

Derivando $\theta^{i}$,

$$
\begin{aligned}
d \theta^{i} & =d\left(v^{j} \omega_{j}^{i}\right) \\
& =d v^{j} \wedge \omega_{j}^{i}+v^{j} d \omega_{j}^{i} \\
& =d v^{j} \wedge \omega_{j}^{i}+v^{j}\left(-\omega_{k}^{i} \wedge \omega_{j}^{k}+\Omega_{j}^{i}\right) \\
& =d v^{j} \wedge \omega_{j}^{i}-v^{j} \omega_{k}^{i} \wedge \omega_{j}^{k}+v^{j} \Omega_{j}^{i} .
\end{aligned}
$$

Observe que

$$
\begin{aligned}
\theta^{j} \wedge \omega_{j}^{i} & =\left(d v^{j}+v^{k} \omega_{k}^{j}\right) \wedge \omega_{j}^{i} \\
& =d v^{j} \wedge \omega_{j}^{i}+v^{k} \omega_{k}^{j} \wedge \omega_{j}^{i} \\
& =d v^{j} \wedge \omega_{j}^{i}-v^{k} \omega_{j}^{i} \wedge \omega_{k}^{j} \\
& =d v^{j} \wedge \omega_{j}^{i}-\overbrace{v^{j} \omega_{k}^{i} \wedge \omega_{j}^{k}}^{k \leftrightarrow j},
\end{aligned}
$$

Segue que

$$
d \theta^{i}=\theta^{j} \wedge \omega_{j}^{i}+v^{j} \Omega_{j}^{i}
$$

Dada outra seção local $t$, cujo domínio tem intersecção não vazia com o domínio de $s$, e sendo $\left\{f_{1}, \cdots, f_{n}\right\}$ referencial ortonormal dado por $t$, por linearidade, a mudança de referenciais é dada pelas funções localmente definidas $a_{j}^{i}: M \rightarrow \mathbb{R}$ que satisfazem $\left(v^{i}\right)_{t}=a_{j}^{i}\left(v^{j}\right)_{s}$ e $\left(\theta^{i}\right)_{t}=a_{j}^{i}\left(\theta^{j}\right)_{s}$. Denotaremos $\pi^{*} v^{i}, \pi^{*} \theta^{i}, \pi^{*} \omega_{j}^{i}$ e $\pi^{*} \Omega_{j}^{i}$ apenas por $v^{i}, \theta^{i}$, $\omega_{j}^{i}$ e $\Omega_{j}^{i}$, isto é, omitiremos o pull-back para o fibrado unitário.

Definição 2.2.1. No contexto anterior, para cada $k \in\left\{0, \cdots, \frac{n}{2}-1\right\}$, definimos

$$
\Phi_{k}=\sum_{\sigma \in S_{n}} \operatorname{sgn}(\sigma) v^{\sigma(1)} \theta^{\sigma(2)} \wedge \cdots \wedge \theta^{\sigma(n-2 k)} \wedge \Omega_{\sigma(n-2 k+2)}^{\sigma(n-2 k+1)} \wedge \cdots \wedge \Omega_{\sigma(n)}^{\sigma(n-1)}
$$




$$
\Psi_{k}=\sum_{\sigma \in S_{n}} \operatorname{sgn}(\sigma) \Omega_{\sigma(2)}^{\sigma(1)} \wedge \theta^{\sigma(3)} \wedge \theta^{\sigma(4)} \wedge \cdots \wedge \theta^{\sigma(n-2 k)} \wedge \Omega_{\sigma(n-2 k+2)}^{\sigma(n-2 k+1)} \wedge \cdots \wedge \Omega_{\sigma(n)}^{\sigma(n-1)}
$$

Alguns fatos sobre $\Phi_{k}$ e $\Psi_{k}: \operatorname{deg}(\Phi)=n-1, \operatorname{deg}(\Psi)=n$, analogamente ao caso de $\Omega$, $\Phi$ e $\Psi$ estão definidas globalmente em $S M$, a mudança de referenciais é dada pela matriz $\left(a_{j}^{i}\right)$ e o efeito dessa troca em $\Phi$ e $\Psi$ é o fator $\operatorname{det}\left(a_{j}^{i}\right)$. Como a variedade é orientada podemos tomar $\operatorname{det}\left(a_{j}^{i}\right)=+1$. Em seus artigos, Chern não deixa claro a origem e intuição das formas $\Phi_{k}$ e $\Psi_{k}$, nas palavras de [Weiping Zhang, 2001, p. 54]:

"It is still mysterious how Chern constructed the forms $\Phi_{k}$ and $\Psi_{k}$ 's".

O artigo [Chern, 1945] apresenta mais detalhes dessas formas, como algumas de suas propriedades.

Derivando $\Phi_{k}$, obtemos

$$
\begin{aligned}
d \Phi_{k} & =\sum_{\sigma \in S_{n}} \operatorname{sgn}(\sigma) d v^{\sigma(1)} \wedge \theta^{\sigma(2)} \wedge \cdots \wedge \theta^{\sigma(n-2 k)} \wedge \Omega_{\sigma(n-2 k+2)}^{\sigma(n-2 k+1)} \wedge \cdots \wedge \Omega_{\sigma(n)}^{\sigma(n-1)}+ \\
& +\underbrace{\sum_{\sigma \in S_{n}} \operatorname{sgn}(\sigma) v^{\sigma(1)} d\left(\theta^{\sigma(2)} \wedge \cdots \wedge \theta^{\sigma(n-2 k)}\right) \wedge \Omega_{\sigma(n-2 k+2)}^{\sigma(n-2 k+1)} \wedge \cdots \wedge \Omega_{\sigma(n)}^{\sigma(n-1)}}_{(1)} \\
& -\underbrace{\sum_{\sigma \in S_{n}} \operatorname{sgn}(\sigma) v^{\sigma(1)} \theta^{\sigma(2)} \wedge \cdots \wedge \theta^{\sigma(n-2 k)} \wedge d\left(\Omega_{\sigma(n-2 k+2)}^{\sigma(n-2 k+1)} \wedge \cdots \wedge \Omega_{\sigma(n)}^{\sigma(n-1)}\right)}_{(2)} .
\end{aligned}
$$

Desenvolvendo (1):

$$
\begin{aligned}
& \sum_{\sigma \in S_{n}} \operatorname{sgn}(\sigma) v^{\sigma(1)} d\left(\theta^{\sigma(2)} \wedge \cdots \wedge \theta^{\sigma(n-2 k)}\right) \wedge \Omega_{\sigma(n-2 k)}^{\sigma(n-2 k+1)} \wedge \cdots \wedge \Omega_{\sigma(n)}^{\sigma(n-1)}= \\
& \sum_{\sigma \in S_{n}} \operatorname{sgn}(\sigma) v^{\sigma(1)}\left(\sum_{j=2}^{n-2 k}(-1)^{j} d \theta^{\sigma(j)} \wedge \theta^{\sigma(2)} \wedge \cdots \wedge \widehat{\theta}^{\sigma(j)} \wedge \cdots \wedge \theta^{\sigma(n-2 k)}\right) \wedge \\
& \wedge \Omega_{\sigma(n-2 k+2)}^{\sigma(n-2 k+1)} \wedge \cdots \wedge \Omega_{\sigma(n)}^{\sigma(n-1)}= \\
& \sum_{\sigma \in S_{n}} \operatorname{sgn}(\sigma) v^{\sigma(1)} d \theta^{\sigma(2)} \wedge \theta^{\sigma(3)} \wedge \cdots \wedge \theta^{\sigma(n-2 k)} \wedge \Omega_{\sigma(n-2 k+2)}^{\sigma(n-2 k+1)} \wedge \cdots \wedge \Omega_{\sigma(n)}^{\sigma(n-1)}+ \\
& \sum_{j=3}^{n-2 k} \sum_{\sigma \in S_{n}} \operatorname{sgn}(\sigma)(-1)^{j} v^{\sigma(1)} d \theta^{\sigma(j)} \wedge \theta^{\sigma(2)} \wedge \cdots \wedge \widehat{\theta}^{\sigma(j)} \wedge \cdots \wedge \theta^{\sigma(n-2 k)} \wedge \\
& \Omega_{\sigma(n-2 k+2)}^{\sigma(n-2 k+1)} \wedge \cdots \wedge \Omega_{\sigma(n)}^{\sigma(n-1)} .
\end{aligned}
$$

No último termo podemos fazer $\sigma=\tau(j 23 \cdots j-1)$, para $j \geq 3$, obtemos $\operatorname{sgn}(\sigma)=$ 


$$
\begin{aligned}
& \operatorname{sgn}(\tau)(-1)^{j} \mathrm{e} \\
& \sum_{\sigma \in S_{n}} \operatorname{sgn}(\sigma) v^{\sigma(1)} d\left(\theta^{\sigma(2)} \wedge \cdots \wedge \theta^{\sigma(n-2 k)}\right) \wedge \Omega_{\sigma(n-2 k+2)}^{\sigma(n-2 k+1)} \wedge \cdots \wedge \Omega_{\sigma(n)}^{\sigma(n-1)}= \\
& \sum_{\sigma \in S_{n}} \operatorname{sgn}(\sigma) v^{\sigma(1)} d \theta^{\sigma(2)} \wedge \theta^{\sigma(3)} \wedge \cdots \wedge \theta^{\sigma(n-2 k)} \wedge \Omega_{\sigma(n-2 k+2)}^{\sigma(n-2 k+1)} \wedge \cdots \wedge \Omega_{\sigma(n)}^{\sigma(n-1)}+ \\
& \sum_{j=3}^{n-2 k} \sum_{\tau \in S_{n}} \operatorname{sgn}(\tau)(-1)^{j+j+2} v^{\tau(1)} d \theta^{\tau(2)} \wedge \theta^{\tau(3)} \wedge \cdots \wedge \theta^{\tau(n-2 k)} \wedge \\
& \Omega_{\sigma(n-2 k+2)}^{\sigma(n-2 k+1)} \wedge \cdots \wedge \Omega_{\sigma(n)}^{\sigma(n-1)} \\
& =(n-2 k-1) \sum_{\sigma \in S_{n}} \operatorname{sgn}(\sigma) v^{\sigma(1)} d \theta^{\sigma(2)} \wedge \theta^{\sigma(3)} \wedge \cdots \wedge \theta^{\sigma(n-2 k)} \wedge \Omega_{\sigma(n-2 k+2)}^{\sigma(n-2 k+1)} \wedge \cdots \wedge \Omega_{\sigma(n)}^{\sigma(n-1)} .
\end{aligned}
$$

Desenvolvendo (2):

$$
\begin{aligned}
& \sum_{\sigma \in S_{n}} \operatorname{sgn}(\sigma) v^{\sigma(1)} \theta^{\sigma(2)} \wedge \cdots \wedge \theta^{\sigma(n-2 k)} \wedge d\left(\Omega_{\sigma(n-2 k+2)}^{\sigma(n-2 k+1)} \wedge \cdots \wedge \Omega_{\sigma(n)}^{\sigma(n-1)}\right)= \\
& \sum_{j=1}^{k} \sum_{\sigma \in S_{n}} \operatorname{sgn}(\sigma) v^{\sigma(1)} \theta^{\sigma(2)} \wedge \cdots \wedge \theta^{\sigma(n-2 k)} d \Omega_{\sigma(n-2 k+2 j)}^{\sigma(n-2 k+2 j-1)} \wedge \Omega_{\sigma(n-2 k+2)}^{\sigma(n-2 k+1)} \wedge \cdots \wedge \widehat{\Omega}_{\sigma(n-2 k+2 j)}^{\sigma(n-2 k+2 j-1)} \\
& \wedge \cdots \wedge \Omega_{\sigma(n)}^{\sigma(n-1)},
\end{aligned}
$$

Fazendo $\sigma=\tau(n-2 k+2 j-1 n-2 k+1 \cdots n-2 k+2 j-3)(n-2 k+2 j n-2 k+2$ $\cdots n-2 k+2 j-2)$, temos $\operatorname{sgn}(\sigma)=\operatorname{sgn}(\tau)$ e

$$
\begin{aligned}
& \sum_{\sigma \in S_{n}} \operatorname{sgn}(\sigma) v^{\sigma(1)} \theta^{\sigma(2)} \wedge \cdots \wedge \theta^{\sigma(n-2 k)} \wedge d\left(\Omega_{\sigma(n-2 k)}^{\sigma(n-2 k+1)} \wedge \cdots \wedge \Omega_{\sigma(n)}^{\sigma(n-1)}\right)= \\
& k \sum_{\sigma \in S_{n}} v^{\sigma(1)} \theta^{\sigma(2)} \wedge \cdots \wedge \theta^{\sigma(n-2 k)} \wedge d \Omega_{\sigma(n-2 k+2)}^{\sigma(n-2 k+1)} \wedge \Omega_{\sigma(n-2 k+4)}^{\sigma(n-2 k+3)} \wedge \cdots \wedge \Omega_{\sigma(n-1)}^{\sigma(n)},
\end{aligned}
$$

portanto,

$$
\begin{aligned}
d \Phi_{k} & =\sum_{\sigma \in S_{n}} \operatorname{sgn}(\sigma) d v^{\sigma(1)} \wedge \theta^{\sigma(2)} \wedge \cdots \wedge \theta^{\sigma(n-2 k)} \wedge \Omega_{\sigma(n-2 k+2)}^{\sigma(n-2 k+1)} \wedge \cdots \wedge \Omega_{\sigma(n)}^{\sigma(n-1)} \\
& +(n-2 k-1) \sum_{\sigma \in S_{n}} \operatorname{sgn}(\sigma) v^{\sigma(1)} d \theta^{\sigma(2)} \wedge \theta^{\sigma(3)} \wedge \cdots \wedge \theta^{\sigma(n-2 k)} \wedge \\
& \wedge \Omega_{\sigma(n-2 k+2)}^{\sigma(n-2 k+1)} \wedge \cdots \wedge \Omega_{\sigma(n)}^{\sigma(n-1)} \\
& -k \sum_{\sigma \in S_{n}} \operatorname{sgn}(\sigma) v^{\sigma(1)} \theta^{\sigma(2)} \wedge \cdots \wedge \theta^{\sigma(n-2 k)} \wedge d \Omega_{\sigma(n-2 k+2)}^{\sigma(n-2 k+1)} \wedge \Omega_{\sigma(n-2 k+4)}^{\sigma(n-2 k+3)} \wedge \cdots \wedge \Omega_{\sigma(n)}^{\sigma(n-1)} .
\end{aligned}
$$


Da definição de $\theta^{i}$, temos $d v^{i}=\theta^{i}-v^{j} \omega_{j}^{i}$. De (2.3) e (2.6) segue que

$$
\begin{aligned}
d \Phi_{k} & =\sum_{\sigma \in S_{n}} \operatorname{sgn}(\sigma)\left(\theta^{\sigma(1)}-v^{j} \omega_{j}^{\sigma(1)}\right) \wedge \theta^{\sigma(2)} \wedge \cdots \wedge \theta^{\sigma(n-2 k)} \wedge \Omega_{\sigma(n-2 k+2)}^{\sigma(n-2 k+1)} \wedge \cdots \wedge \Omega_{\sigma(n)}^{\sigma(n-1)} \\
& +(n-2 k-1) \sum_{\sigma \in S_{n}} \operatorname{sgn}(\sigma) v^{\sigma(1)}\left(\theta^{j} \wedge \omega_{j}^{\sigma(2)}+v^{j} \Omega_{j}^{\sigma(2)}\right) \wedge \theta^{\sigma(3)} \wedge \cdots \wedge \theta^{\sigma(n-2 k)} \\
& \wedge \Omega_{\sigma(n-2 k+2)}^{\sigma(n-2 k+1)} \wedge \cdots \wedge \Omega_{\sigma(n)}^{\sigma(n-1)} \\
& -k \sum_{\sigma \in S_{n}} \operatorname{sgn}(\sigma) v^{\sigma(1)} \theta^{\sigma(2)} \wedge \cdots \wedge \theta^{\sigma(n-2 k)} \wedge \\
& \left(-\Omega_{s}^{\sigma(n-2 k+1)} \wedge \omega_{\sigma(n-2 k+2)}^{s}-\omega_{s}^{\sigma(n-2 k+1)} \wedge \Omega_{\sigma(n-2 k+2)}^{s}\right) \wedge \Omega_{\sigma(n-2 k+4)}^{\sigma(n-2 k+3)} \wedge \cdots \wedge \Omega_{\sigma(n)}^{\sigma(n-1)} .
\end{aligned}
$$

Seja $p \in M$ e tome um referencial geodésico em torno de $p$. Então, nesse referencial, $\left(\omega_{j}^{i}\right)_{p}=0$, assim

$$
\begin{aligned}
d \Phi_{k} & =\sum_{\sigma \in S_{n}} \operatorname{sgn}(\sigma) \theta^{\sigma(1)} \wedge \cdots \wedge \theta^{\sigma(n-2 k)} \wedge \Omega_{\sigma(n-2 k+2)}^{\sigma(n-2 k+1)} \wedge \cdots \wedge \Omega_{\sigma(n)}^{\sigma(n-1)}+ \\
& (n-2 k-1) \sum_{\sigma \in S_{n}} \operatorname{sgn}(\sigma) v^{\sigma(1)} v^{j} \Omega_{j}^{\sigma(2)} \wedge \theta^{\sigma(3)} \wedge \cdots \wedge \theta^{\sigma(n-2 k)} \wedge \\
& \wedge \Omega_{\sigma(n-2 k+2)}^{\sigma(n-2 k+1)} \wedge \cdots \wedge \Omega_{\sigma(n)}^{\sigma(n-1)} .
\end{aligned}
$$

Como a forma $\Phi_{k}$ é intrínseca, podemos assumir que vale sempre essa representação. Note que

$$
\begin{aligned}
\Psi_{k-1} & =\sum_{\sigma \in S_{n}} \operatorname{sgn}(\sigma) \Omega_{\sigma(2)}^{\sigma(1)} \wedge \theta^{\sigma(3)} \wedge \theta^{\sigma(4)} \wedge \cdots \wedge \theta^{\sigma(n-2 k+2)} \wedge \Omega_{\sigma(n-2 k+4)}^{\sigma(n-2 k+3)} \wedge \cdots \wedge \Omega_{\sigma(n)}^{\sigma(n-1)} \\
& =\sum_{\sigma \in S_{n}} \operatorname{sgn}(\sigma) \theta^{\sigma(3)} \wedge \theta^{\sigma(4)} \wedge \cdots \wedge \theta^{\sigma(n-2 k-2)} \wedge \Omega_{\sigma(2)}^{\sigma(1)} \wedge \Omega_{\sigma(n-2 k)}^{\sigma(n-2 k-1)} \wedge \cdots \wedge \Omega_{\sigma(n)}^{\sigma(n-1)} \\
& =\sum_{\sigma \in S_{n}} \operatorname{sgn}(\sigma) \theta^{\sigma(1)} \wedge \cdots \wedge \theta^{\sigma(n-2 k)} \wedge \Omega_{\sigma(n-2 k+2)}^{\sigma(n-2 k+1)} \wedge \cdots \wedge \Omega_{\sigma(n)}^{\sigma(n-1)}
\end{aligned}
$$

concluímos

$d \Phi_{k}=\Psi_{k-1}+N \sum_{\sigma \in S_{n}} \operatorname{sgn}(\sigma) v^{\sigma(1)} v^{j} \Omega_{j}^{\sigma(2)} \wedge \theta^{\sigma(3)} \wedge \cdots \wedge \theta^{\sigma(n-2 k)} \wedge \Omega_{\sigma(n-2 k+2)}^{\sigma(n-2 k+1)} \wedge \cdots \wedge \Omega_{\sigma(n)}^{\sigma(n-1)}$

em que $N=(n-2 k-1)$. Defina

$$
\left\{\begin{array}{l}
P_{k}=\sum_{\sigma \in S_{n}} \operatorname{sgn}(\sigma)\left(v^{\sigma(1)}\right)^{2} \Omega_{\sigma(2)}^{\sigma(1)} \theta^{\sigma(3)} \wedge \cdots \wedge \theta^{\sigma(n-2 k)} \wedge \Omega_{\sigma(n-2 k+2)}^{\sigma(n-2 k+1)} \wedge \cdots \wedge \Omega_{\sigma(n)}^{\sigma(n-1)} \\
\Sigma_{k}=\sum_{\sigma \in S_{n}} \operatorname{sgn}(\sigma) v^{\sigma(1)} v^{\sigma(3)} \Omega_{\sigma(3)}^{\sigma(2)} \wedge \theta^{\sigma(3)} \wedge \cdots \wedge \theta^{\sigma(n-2 k)} \wedge \Omega_{\sigma(n-2 k+2)}^{\sigma(n-2 k+1)} \wedge \cdots \wedge \Omega_{\sigma(n)}^{\sigma(n-1)} \\
T_{k}=\sum_{\sigma \in S_{n}} \operatorname{sgn}(\sigma)\left(v^{\sigma(3)}\right)^{2} \Omega_{\sigma(2)}^{\sigma(1)} \theta^{\sigma(3)} \wedge \cdots \wedge \theta^{\sigma(n-2 k)} \wedge \Omega_{\sigma(n-2 k+2)}^{\sigma(n-2 k+1)} \wedge \cdots \wedge \Omega_{\sigma(n)}^{\sigma(n-1)} .
\end{array}\right.
$$


Em $d \Phi_{k}$ temos o termo $v^{j} \Omega_{j}^{\sigma(2)}$; para $j=\sigma(1)$, temos justamente $P_{k}$, para $j=\sigma(3)$ aparece $\Sigma_{k}$; e para $j=\sigma(2)$, o fator $\Omega_{\sigma(2)}^{\sigma(2)}$ é nulo. Resumidamente,

$$
\begin{aligned}
d \Phi_{k} & =\Psi_{k-1}+(n-2 k-1)\left(P_{k}+\Sigma_{k}+\right. \\
& \sum_{\sigma \in S_{n}} \operatorname{sgn}(\sigma) \sum_{j \neq \sigma(1), \sigma(2), \sigma(3)} v^{\sigma(1)} v^{j} \Omega_{j}^{\sigma(2)} \theta^{\sigma(3)} \wedge \cdots \wedge \theta^{\sigma(n-2 k)} \wedge \\
& \left.\wedge \Omega_{\sigma(n-2 k+2)}^{\sigma(n-2 k+1)} \wedge \cdots \wedge \Omega_{\sigma(n)}^{\sigma(n-1)}\right) .
\end{aligned}
$$

Se $j=\sigma(n-2 k+l)$, para algum $l \geq 1$, então alguns índice inferiores se repetem em $\Omega_{\sigma(n-2 k+2)}^{\sigma(n-2 k+1)} \wedge \cdots \wedge \Omega_{\sigma(n)}^{\sigma(n-1)}$, portanto o é nulo. Resta o caso $j=\sigma(\alpha), \alpha \in\{4, \cdots, n-2 k\}$. Considere $\sigma=\tau(\alpha 3)$. Então

$$
\begin{aligned}
& \sum_{\tau \in S_{n}} \sum_{j}-\operatorname{sgn}(\tau) v^{\tau(1)} v^{\tau(3)} \Omega_{\tau(3)}^{\tau(2)} \wedge \theta^{\tau\left(\sigma^{-1}(j)\right)} \wedge \theta^{\tau(4)} \wedge \cdots \wedge \underbrace{\theta^{\tau(3)}}_{\text {posição } \alpha} \wedge \cdots \wedge \theta^{\tau(n-2 k)} \\
\wedge & \Omega_{\tau(n-2 k+2)}^{\tau(n-2 k+1)} \wedge \cdots \wedge \Omega_{\tau(n)}^{\tau(n-1)} \\
= & \sum_{\tau \in S_{n}} \sum_{j}-\operatorname{sgn}(\tau)(-1)^{\alpha-3}(-1)^{\alpha-4} v^{\tau(1)} v^{\tau(3)} \Omega_{\tau(3)}^{\tau(2)} \wedge \theta^{\tau(3)} \wedge \theta^{\tau(4)} \wedge \cdots \wedge \theta^{\tau(n-2 k)} \\
\wedge & \Omega_{\tau(n-2 k+2)}^{\tau(n-2 k+1)} \wedge \cdots \wedge \Omega_{\tau(n)}^{\tau(n-1)} \\
= & \sum_{\tau \in S_{n}} \sum_{j} \operatorname{sgn}(\tau) v^{\tau(1)} v^{\tau(3)} \Omega_{\tau(3)}^{\tau(2)} \wedge \theta^{\tau(3)} \wedge \theta^{\tau(4)} \wedge \cdots \wedge \theta^{\tau(n-2 k)} \\
\wedge & \Omega_{\tau(n-2 k+2)}^{\tau(n-2 k+1)} \wedge \cdots \wedge \Omega_{\tau(n)}^{\tau(n-1)} \\
= & (n-2 k-3) \Sigma_{k} .
\end{aligned}
$$

Portanto

$$
d \Phi_{k}=\Psi_{k-1}+(n-2 k-1)\left(P_{k}+(n-2 k-2) \Sigma_{k}\right) .
$$

Desenvolvendo $P_{k}$,

$$
\begin{aligned}
P_{k} & =\sum_{\sigma \in S_{n}} \operatorname{sgn}(\sigma)\left(v^{\sigma(1)}\right)^{2} \Omega_{\sigma(2)}^{\sigma(1)} \theta^{\sigma(3)} \wedge \cdots \wedge \theta^{\sigma(n-2 k)} \wedge \Omega_{\sigma(n-2 k+2)}^{\sigma(n-2 k+1)} \wedge \cdots \wedge \Omega_{\sigma(n)}^{\sigma(n-1)} \\
& =\sum_{\sigma \in S_{n}} \operatorname{sgn}(\sigma)\left(1-\left(v^{\sigma(2)}\right)^{2}-\cdots-\left(v^{\sigma(n)}\right)^{2}\right) \Omega_{\sigma(2)}^{\sigma(1)} \theta^{\sigma(3)} \wedge \cdots \wedge \theta^{\sigma(n-2 k)} \\
& \wedge \Omega_{\sigma(n-2 k+2)}^{\sigma(n-2 k+1)} \wedge \cdots \wedge \Omega_{\sigma(n)}^{\sigma(n-1)} \\
& =\Psi_{k}-P_{k}-T_{k}-\sum_{j=4}^{n} \sum_{\sigma \in S_{n}} \operatorname{sgn}(\sigma)\left(v^{\sigma(j)}\right)^{2} \Omega_{\sigma(2)}^{\sigma(1)} \wedge \theta^{\sigma(3)} \wedge \cdots \wedge \theta^{\sigma(n-2 k)} \wedge \\
& \Omega_{\sigma(n-2 k+2)}^{\sigma(n-2 k+1)} \wedge \cdots \wedge \Omega_{\sigma(n)}^{\sigma(n-1)} .
\end{aligned}
$$

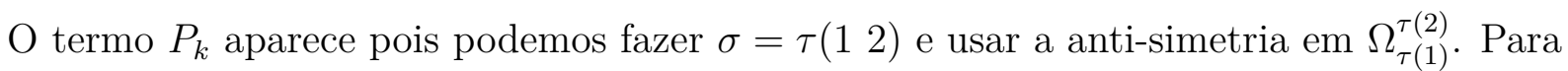


$j \in\{4, \cdots, n-2 k\}$ fazemos $\sigma=\tau(j 3)$, então

$$
\begin{aligned}
P_{k} & =\Psi_{k}-P_{k}-T_{k}-(n-2 k-1) T_{k} \\
& -\sum_{j=n-2 k+1}^{n} \sum_{\sigma \in S_{n}} \operatorname{sgn}(\sigma)\left(v^{\sigma(j)}\right)^{2} \Omega_{\sigma(2)}^{\sigma(1)} \wedge \theta^{\sigma(3)} \wedge \cdots \wedge \theta^{\sigma(n-2 k)} \wedge \Omega_{\sigma(n-2 k+2)}^{\sigma(n-2 k+1)} \wedge \cdots \wedge \Omega_{\sigma(n)}^{\sigma(n-1)}
\end{aligned}
$$

Para o termo $\sum_{j=n-2 k+1}^{n} \sum_{\sigma \in S_{n}} \operatorname{sgn}(\sigma)\left(v^{\sigma(j)}\right)^{2} \Omega_{\sigma(2)}^{\sigma(1)} \wedge \theta^{\sigma(3)} \wedge \cdots \wedge \theta^{\sigma(n-2 k)} \wedge \Omega_{\sigma(n-2 k+2)}^{\sigma(n-2 k+1)} \wedge \cdots \wedge$ $\Omega_{\sigma(n)}^{\sigma(n-1)}$ dividimos $j$ em pares e ímpares; para os ímpares fazemos $\sigma=\tau(j 1)(j+12)$; para os pares, $\sigma=(j-12)(j 1)$, e toda a soma torna-se $2 k P_{k} ;$ assim $2 P_{k}=\Psi_{k}-(n-$ $2 k-2) T_{k}-2 k P_{k}$, isto é,

$$
\Psi_{k}=2(k+1) P_{k}+(n-2 k-2) T_{k}
$$

Por fim vamos desenvolver $\Sigma_{k}$. Em $\Sigma_{k}$ podemos utilizar (2.5) para os termos $v^{\sigma(3)}$ e $\theta^{\sigma(3)}$, isto é,

$$
\begin{aligned}
\Sigma_{k} & =\sum_{\sigma \in S_{n}} \operatorname{sgn}(\sigma) v^{\sigma(1)} v^{\sigma(3)} \Omega_{\sigma(3)}^{\sigma(2)} \wedge \theta^{\sigma(3)} \wedge \cdots \wedge \theta^{\sigma(n-2 k)} \wedge \Omega_{\sigma(n-2 k+2)}^{\sigma(n-2 k+1)} \wedge \cdots \wedge \Omega_{\sigma(n)}^{\sigma(n-1)} \\
& =\sum_{\sigma \in S_{n}} \operatorname{sgn}(\sigma) v^{\sigma(1)} \Omega_{\sigma(3)}^{\sigma(2)} \wedge\left(-\sum_{j \neq 3} v^{\sigma(j)} \theta^{\sigma(j)}\right) \theta^{\sigma(4)} \wedge \cdots \wedge \theta^{\sigma(n-2 k)} \wedge \\
& \Omega_{\sigma(n-2 k+2)}^{\sigma(n-2 k+1)} \wedge \cdots \wedge \Omega_{\sigma(n)}^{\sigma(n-1)} ;
\end{aligned}
$$

para $j=1$, podemos fazer $\sigma=\tau(31)$, e usando a anti-simetria em $\Omega_{\sigma(2)}^{\sigma(3)}$, obtemos $T_{k}$, para $j=2$, trocamos $\sigma=\tau(32)$ e a anti-simetria em $\Omega_{\sigma(2)}^{\sigma(3)}$ nos da o termo $-\Sigma_{k}$; para $j \in\{4, \cdots, n-2 k\}$, a forma $\theta^{\sigma(j)}$ se repete, logo o termo todo é nulo, assim

$$
\begin{aligned}
\Sigma_{k} & =T_{k}-\Sigma_{k}-\sum_{j=n-2 k+1}^{n} \sum_{\sigma \in S_{n}} \operatorname{sgn}(\sigma) v^{\sigma(1)} v^{\sigma(j)} \Omega_{\sigma(3)}^{\sigma(2)} \wedge \theta^{\sigma(j)} \wedge \theta^{\sigma(4)} \wedge \cdots \wedge \theta^{\sigma(n-2 k)} \\
& \wedge \Omega_{\sigma(n-2 k+2)}^{\sigma(n-2 k+1)} \wedge \cdots \wedge \Omega_{\sigma(n)}^{\sigma(n-1)} .
\end{aligned}
$$

Novamente separando $j$ em ímpares e pares, e mudando as permutações obtemos

$$
\sum_{\sigma \in S_{n}} \operatorname{sgn}(\sigma) v^{\sigma(1)} v^{\sigma(j)} \Omega_{\sigma(3)}^{\sigma(2)} \wedge \theta^{\sigma(j)} \wedge \theta^{\sigma(4)} \wedge \cdots \wedge \theta^{\sigma(n-2 k)} \wedge \Omega_{\sigma(n-2 k+2)}^{\sigma(n-2 k+1)} \wedge \cdots \wedge \Omega_{\sigma(n)}^{\sigma(n-1)}=2 k \Sigma_{k} .
$$

Portanto $\Sigma_{k}=T_{k}-(2 k+1) \Sigma_{k}$, isto é,

$$
T_{k}=2(k+1) \Sigma_{k}
$$

Proposição 2.2.2. Para cada $k \in\left\{0, \cdots, \frac{n}{2}-1\right\}$,

$$
d \Phi_{k}=\Psi_{k-1}+\frac{n-2 k-1}{2(k+1)} \Psi_{k}
$$

em que $\Psi_{-1}:=0$. 
Demonstração. Por (2.8), (2.9) e (2.10) vale

$$
\begin{aligned}
d \Phi_{k} & =\Psi_{k-1}+(n-2 k-1)\left(P_{k}+(n-2 k-2) \Sigma_{k}\right) \\
& =\Psi_{k-1}+(n-2 k-1)\left(\frac{\Psi_{k}-(n-2 k-2) T_{k}}{2(k+1)}+(n-2 k-2) \frac{T_{k}}{2(k+1)}\right) \\
& =\Psi_{k-1}+(n-2 k-1)(\frac{\Psi_{k}}{2(k+1)} \underbrace{-(n-2 k-2) \frac{T_{k}}{2(k+1)}+(n-2 k-2) \frac{T_{k}}{2(k+1)}}_{0}) \\
& =\Psi_{k-1}+\frac{n-2 k-1}{2(k+1)} \Psi_{k} .
\end{aligned}
$$

Observação 2.2.3. Podemos isolar cada $\Psi_{k}=\frac{2(k+1)}{n-2 k-1}\left(d \Phi_{k}-\Psi_{k-1}\right)$, mas

$$
\Psi_{k-1}=\frac{2 k}{n-2 k+1}\left(d \Phi_{k-1}-\Psi_{k-2}\right),
$$

então $\Psi_{k}=\frac{2(k+1)}{n-2 k-1} d \Phi_{k}-\frac{2(k+1)}{n-2 k-1}\left(\frac{2 k}{n-2 k+1}\left(d \Phi_{k-1}-\Psi_{k-2}\right)\right)$. Recursivamente,

$$
\Psi_{k}=\sum_{t=0}^{k}\left(\frac{(-1)^{k-t} 2^{k+1-t}(k+1) \cdot k \cdots(t+1)}{(n-2 k-1) \cdots(n-2 t-1)} d \Phi_{t}\right) .
$$

Por definição $\Psi_{\frac{n}{2}-1}=\sum_{\sigma \in S_{n}} \operatorname{sgn}(\sigma) \Omega_{\sigma(2)}^{\sigma(1)} \wedge \cdots \wedge \Omega_{\sigma(n)}^{\sigma(n-1)}$. Então $-\pi^{*}(\Omega)=\frac{(-1)^{\frac{n}{2}-1}}{2^{n} \pi^{\frac{n}{2}}\left(\frac{n}{2}\right) !} \Psi_{\frac{n}{2}-1}$. De $(2.12)$

$$
-\pi^{*}(\Omega)=\frac{1}{\pi^{\frac{n}{2}}} \sum_{t=0}^{\frac{n}{2}-1} \frac{(-1)^{t}}{2^{\frac{n}{2}+t} t !} \frac{1}{1 \cdots(n-2 t-1)} d \Phi_{t},
$$

Lema 2.2.4. (Transgressão de $\Omega$ ). Existe $\Pi \in \Omega^{n-1}(S M)$ tal que $d \Pi=-\pi^{*}(\Omega)$.

Demonstração. Defina $\Pi:=\frac{1}{\pi^{\frac{n}{2}}} \sum_{t=0}^{\frac{n}{2}-1} \frac{(-1)^{t}}{2^{\frac{n}{2}+t} t !} \frac{1}{1 \cdots(n-2 t-1)} \Phi_{t}$. De $(2.13)$ segue $-\pi^{*}(\Omega)=$ $d \Pi$

\subsection{Demonstração da fórmula de Gauss-Bonnet-}

\section{Chern}

Nesta seção apresentaremos uma adaptação da demonstração do Teorema 2.1.5 dada por S.-S. Chern no artigo A Simple Intrinsic Proof of the Gauss-Bonnet Formula for Closed Riemannian Manifolds [Chern, 1944]. Para essa adaptação usamos essencialmente os livros [Raoul Bott, 1995] e [Weiping Zhang, 2001]. 
Demonstração. Theorem 2.1.5. Seja $f: M \rightarrow \mathbb{R}$ função de Morse, [Guillemin, 1974]. A função $f$ tem pontos críticos isolados, digamos $\left\{x_{1}, \cdots, x_{s}\right\} \subset M$. Para cada $i \in$ $\{1, \cdots, s\}$, tome $W_{i}$ aberto contrátil em torno de $x_{i}$ contido em uma bola geodésica de raio $r_{i}$. Defina $r=\min \left\{r_{1}, \cdots, r_{n}\right\}, M_{r}=M \backslash \bigcup_{i=1}^{s} B_{r}\left(x_{i}\right)$, em que $B_{r}\left(x_{i}\right)$ é bola geodésica em torno de $x_{i}$ com raio $r$, e $X: M_{r} \rightarrow S M$, por $X(p)=\frac{V(p)}{\|V(p)\|_{g}}$, em que $V(p)=\left(\nabla_{g} f\right)(p)$. Da Transgressão temos $\pi^{*}(\Omega)=-d \Pi$. Como $\pi \circ X=I d_{M_{r}}$, então $X^{*}(d \Pi)=-\Omega$ e vale

$$
\begin{aligned}
\int_{M} \Omega & =\lim _{r \rightarrow 0} \int_{M_{r}} \Omega \\
& =-\lim _{r \rightarrow 0} \int_{M_{r}} X^{*}(d \Pi) \\
& =-\lim _{r \rightarrow 0} \int_{\partial M_{r}} X^{*} \Pi
\end{aligned}
$$

Na última igualdade usamos o Teorema de Stokes.

Afirmação:

$$
H_{d R}^{n-1}\left(\left.S M\right|_{W_{i}}\right)=\operatorname{span}\{\Pi\}
$$

De fato, como $W_{i}$ é contrátil,

$$
S M_{x_{i}} \cong\left\{x_{i}\right\} \times \mathbb{S}^{n-1} \cong{ }_{\text {homot. }} W_{i} \times\left.\mathbb{S}^{n-1} \cong S M\right|_{W_{i}}
$$

$\operatorname{logo} \operatorname{dim}\left(H_{d R}^{n-1}\left(\left.S M\right|_{W_{i}}\right)\right)=\operatorname{dim}\left(H^{n-1}\left(S M_{x_{i}}\right)\right)=1$. Então basta verificar que $\int_{S M_{x_{i}}} \Pi \neq 0$. Por definição,

$$
\int_{S M_{x_{i}}} \Pi=\frac{1}{\pi^{\frac{n}{2}}} \sum_{t=0}^{\frac{n}{2}-1}(-1)^{t} \int_{S M_{x_{i}}} \frac{1}{1 \cdot 3 \cdots(n-2 t-1) t ! 2^{\frac{n}{2}+t}} \Phi_{t} .
$$

Calculemos primeiro cada $\int_{S M_{x_{i}}} \Phi_{t}$,

$$
\int_{S M_{x_{i}}} \Phi_{t}=\int_{S M_{x_{i}}} \sum_{\sigma \in S_{n}} \operatorname{sgn}(\sigma) v^{\sigma(1)} \theta^{\sigma(2)} \wedge \cdots \wedge \theta^{\sigma(n-2 t)} \wedge \Omega_{\sigma(2-2 t+2)}^{\sigma(n-2 t+1)} \wedge \cdots \wedge \Omega_{\sigma(n)}^{\sigma(n-1)} .
$$

Em coordenadas geodésicas em torno de $x_{i}$, temos $\theta^{i}=d v^{i}$ e $\Omega_{j}^{i}=d \omega_{j}^{i}$. Assuma $t>0$.

$$
\begin{aligned}
\int_{S M_{x_{i}}} \Phi_{t} & =\int_{S M_{x_{i}}} \sum_{\sigma \in S_{n}} \operatorname{sgn}(\sigma) v^{\sigma(1)} d v^{\sigma(2)} \wedge \cdots \wedge d v^{\sigma(n-2 t)} \wedge d \omega_{\sigma(n-2 t+2)}^{\sigma(n-2 t+1)} \wedge \cdots \wedge d \omega_{\sigma(n)}^{\sigma(n-1)} \\
& =\sum_{\sigma \in S_{n}} \int_{S M_{x_{i}}} \alpha_{\sigma} \wedge d \omega_{\sigma(n)}^{\sigma(n-1)}
\end{aligned}
$$

em que $\alpha_{\sigma}=\operatorname{sgn}(\sigma) v^{\sigma(1)} d v^{\sigma(2)} \wedge \cdots \wedge d v^{\sigma(n-2 t)} \wedge d \omega_{\sigma(n-2 t+2)}^{\sigma(n-2 t+1)} \wedge \cdots \wedge d \omega_{\sigma(n-2)}^{\sigma(n-3)}$. Note que $\operatorname{deg}\left(\alpha_{\sigma}\right)=n-3$. Como $n$ é par, $(-1)^{n-3}=-1$, Note que

$$
d\left(\alpha_{\sigma} \wedge \omega_{\sigma(n)}^{\sigma(n-1)}\right)=d \alpha_{\sigma} \wedge \omega_{\sigma(n)}^{\sigma(n-1)}-\alpha \wedge d \omega_{\sigma(n)}^{\sigma(n-1)}
$$


assim, por um lado,

$$
\int_{S M_{x_{i}}} d\left(\alpha \wedge \omega_{\sigma(n)}^{\sigma(n-1)}\right)=\int_{\partial S M_{x_{i}}} \alpha_{\sigma} \wedge \omega_{\sigma(n)}^{\sigma(n-1)}=0
$$

onde usamos Stokes e o fato que $\partial S M_{x_{i}}=\emptyset$. Por outro lado, de (2.15)

$$
\int_{S M_{x_{i}}} d\left(\alpha \wedge \omega_{\sigma(n)}^{\sigma(n-1)}\right)=\int_{S M_{x_{i}}} d \alpha_{\sigma} \wedge \omega_{\sigma(n)}^{\sigma(n-1)}-\int_{S M_{x_{i}}} \alpha \wedge d \omega_{\sigma(n)}^{\sigma(n-1)}
$$

Como $\omega_{j}^{i}=0$ em $x_{i}$, segue $\int_{S M_{x_{i}}} \alpha \wedge d \omega_{\sigma(n)}^{\sigma(n-1)}=0$, isto é $\int_{S M_{x_{i}}} \Phi_{t}=0$ para $t>0$. Concluímos que

$$
\int_{S M_{x_{i}}} \Pi=\frac{1}{1 \cdot 3 \cdots(n-1) 2^{\frac{n}{2}} \pi^{\frac{n}{2}}} \int_{S M_{x_{i}}} \Phi_{0},
$$

Para explicitar $\int_{S M_{x_{i}}} \Phi_{0}$ no referencial geodésico, precisamos do seguinte lema.

Lema 2.3.1. Sejam $t \in \mathbb{N}$, e $\theta^{1}, \cdots, \theta^{t} \in \Omega^{1}(M)$. Então $\sum_{\sigma \in S_{t}} \operatorname{sgn}(\sigma) \theta^{\sigma(1)} \wedge \cdots \wedge \theta^{\sigma(t)}=$ $t ! \theta^{1} \wedge \cdots \wedge \theta^{t}$.

Demonstração. Por indução em $t$.

Para $t=1$ nada temos a provar.

Supondo válido para $t-1$, temos

$$
\begin{aligned}
\sum_{\sigma \in S_{t}} \operatorname{sgn}(\sigma) \theta^{\sigma(1)} \wedge \cdots \wedge \theta^{\sigma(t)} & =\sum_{i=1}^{t} \theta^{i} \wedge \sum_{\sigma \in S_{t}, \sigma(1)=i} \operatorname{sgn}(\sigma) \theta^{\sigma(2)} \wedge \cdots \wedge \theta^{\sigma(t)} \\
& \stackrel{\sigma=\tau(1 i)}{=} \sum_{i=1}^{t} \theta^{i} \wedge \sum_{\sigma \in S_{t}, \tau(i)=i} \operatorname{sgn}(\tau(1 i)) \theta_{\tau(2)} \wedge \cdots \\
& \wedge \theta^{\tau(1)} \wedge \cdots \wedge \theta^{\tau(t)} \\
& =\sum_{i=1}^{t} \theta^{i} \wedge \sum_{\sigma \in S_{t}, \tau(i)=i} \operatorname{sgn}(\tau(1 i))(-1)^{i-2} \theta^{\tau(1)} \wedge \cdots \\
& \wedge \widehat{\theta^{i}} \wedge \cdots \wedge \theta^{\tau(t)} \\
& =\sum_{i=1}^{t} \theta^{i} \wedge \sum_{\sigma \in S_{t}, \tau(i)=i} \operatorname{sgn}(\tau)(-1)^{i-1} \theta^{\tau(1)} \wedge \cdots \\
& \wedge \widehat{\theta^{i}} \wedge \cdots \wedge \theta^{\tau(t)} \\
& \stackrel{i n d .}{=} \sum_{i=1}^{t} \theta^{i}(-1)^{i-1} \wedge(t-1) ! \theta^{1} \wedge \cdots \wedge \hat{\theta}^{i} \wedge \cdots \wedge \theta^{t} \\
& =t ! \theta^{1} \wedge \cdots \wedge \theta^{t} .
\end{aligned}
$$


Proposição 2.3.2. $\Phi_{0}=(n-1) ! \sum_{i=1}^{n}(-1)^{i-1} v^{i} \theta^{1} \wedge \cdots \widehat{\theta}^{i} \wedge \cdots \theta^{n}$.

Demonstração. Usando o lema anterior,

$$
\begin{aligned}
\Phi_{0} & =\sum_{\sigma \in S_{n}} \operatorname{sgn}(\sigma) v^{\sigma(1)} \theta^{\sigma(2)} \wedge \cdots \wedge \theta^{\sigma(n)} \\
& =\sum_{i=1}^{n} v^{i} \sum_{\sigma \in S_{n}, \sigma(1)=i} \operatorname{sgn}(\sigma) \theta^{\sigma(2)} \wedge \cdots \wedge \theta^{\sigma(n)} \\
& \stackrel{\sigma=\tau(1 i)}{=} \sum_{i=1}^{n} v_{i} \sum_{\tau \in S_{n}, \tau(i)=i} \operatorname{sgn}(\tau(1 i)) \theta^{\tau(2)} \wedge \cdots \wedge \theta^{\tau(1)} \wedge \theta^{\sigma(i+1)} \wedge \cdots \wedge \theta^{\tau(n)} \\
& =\sum_{i=1}^{n} v_{i}(-1)^{i-1} \sum_{\tau \in S_{n}, \tau(i)=i} \operatorname{sgn}(\tau) \theta^{\tau(1)} \wedge \cdots \wedge \widehat{\theta}^{i} \wedge \cdots \wedge \theta^{\tau(n)} \\
& \stackrel{\text { lema }}{=}(n-1) ! \sum_{i=1}^{n}(-1)^{i-1} v_{i} \theta^{1} \cdots \wedge \widehat{\theta}^{i} \wedge \cdots \wedge \theta^{n} .
\end{aligned}
$$

Voltando para $\int_{S M_{x_{i}}} \Phi_{0}$

$$
\begin{aligned}
\int_{S M_{x_{i}}} \Phi_{0} & =(n-1) ! \int_{S M_{x_{i}}} \sum_{i=1}^{n}(-1)^{i-1} v^{i} d v^{1} \wedge \cdots \wedge \hat{d v^{i}} \wedge \cdots \wedge d v^{n} \\
& =(n-1) ! \frac{2 \pi^{\frac{n}{2}}}{\left(\frac{n}{2}-1\right) !}
\end{aligned}
$$

portanto $\int_{S M_{x_{i}}} \Pi=\frac{1}{1 \cdot 3 \cdots(n-1) 2^{\frac{n}{2}} \pi^{\frac{n}{2}}}(n-1) ! \cdot \frac{2 \pi^{\frac{n}{2}}}{\left(\frac{n}{2}-1\right) !}=\frac{(n-1) !}{1 \cdot 3 \cdots(n-1) 2^{\frac{n}{2}-1}\left(\frac{n}{2}-1\right) !}$, mas $\left(\frac{n}{2}-1\right) !=\left(\frac{n}{2}-1\right) \cdot\left(\frac{n}{2}-2\right) \cdot\left(\frac{n}{2}-3\right) \cdots 1$. Como temos o fator $2^{\frac{n}{2}-1}$ multiplicando, $2^{\frac{n}{2}-1}\left(\frac{n}{2}-1\right) !=(n-2) \cdot(n-4) \cdots 2$. Segue

$$
1 \cdot 3 \cdots(n-1) 2^{\frac{n}{2}-1}\left(\frac{n}{2}-1\right) !=(1 \cdot 3 \cdots(n-1))((n-2) \cdot(n-4) \cdots 2)=(n-1) ! .
$$

$\operatorname{Logo} \int_{S M_{x_{i}}} \Pi=1$, o que prova a afirmação (2.14).

Seja $\rho: W_{i} \times \mathbb{S}^{n-1} \rightarrow \mathbb{S}^{n-1}$ a projeção, e tome $\sigma \in H_{d R}^{n-1}\left(\mathbb{S}^{n-1}\right)$ tal que $\int_{\mathbb{S}^{n-1}} \sigma=1$. Temos $\int_{S M_{x_{i}}} \rho^{*} \sigma=1$ e $\int_{S M_{x_{i}}} \Pi=1$, portanto existe $\tau \in \Omega^{n-2}\left(S M_{x_{i}}\right)$ tal que $\rho^{*} \sigma-\Pi=d \tau$, uma vez que em $S M_{x_{i}}$ uma $(n-1)$-forma é exata se, e somente se, sua integral sobre $S M_{x_{i}}$ é zero. Agora

$$
\int_{\partial M_{r}} X^{*}\left(\rho^{*} \sigma-\Pi\right)=\int_{\emptyset} X^{*} \tau=0
$$


Como a orientação de $\partial M_{r}$ é oposta a de $\bigcup B_{r}\left(x_{i}\right)$,

$$
\begin{aligned}
\int_{\partial M_{r}} X^{*} \Pi & =\int_{\partial M_{r}}(\rho \circ X)^{*} \sigma \\
& =-\sum_{i=1}^{s} i n d_{V}\left(x_{i}\right) \int_{\mathbb{S}^{n-1}} \sigma \\
& =-\sum_{i=1}^{s} i n d_{V}\left(x_{i}\right) .
\end{aligned}
$$

Concluímos que $\int_{M} \Omega=-\lim _{r \rightarrow 0} \int_{\partial M_{r}} X^{*} \Pi=-\left(-\sum_{i=1}^{s} i n d_{V}\left(x_{i}\right)\right)=\sum_{i=1}^{s} i n d_{V}\left(x_{i}\right)=\chi(M)$, em que a última igualdade segue do Teorema 2.1.3. 


\section{Teoria de Cartan-Kähler}

\subsection{Cartan-Kähler para tableaux}

Esta seção desenvolverá o conceito de tableau que em palavras é o espaço (linear) das possíveis derivadas parciais de uma EDP de primeira ordem, homogênea e com coeficientes constantes. Codificada essa EDP através de tableaux e inspirados no problema de Cauchy, enunciaremos o Teorema de Cartan-Kähler para tableaux.

Sejam $V$ e $W$ espaços vetoriais reais munidos de produtos internos e $b=\left(v_{1}, \cdots, v_{n}\right)$, $q=\left(w_{1}, \cdots, w_{s}\right)$ respectivas bases ordenadas. Uma EDP de primeira ordem, homogênea e com coeficientes constantes para aplicações de $f: V \rightarrow W$, em coordenadas, é dada por

$$
B_{a}^{r i} \frac{\partial u^{a}}{\partial x^{i}}=0
$$

para $r \in\{1, \cdots, R\}$ onde $B_{a}^{r i}$ são constantes.

Exemplo 3.1.1. (Equação de Cauchy-Riemann). Seja $f=(u, v): \mathbb{C} \rightarrow \mathbb{C}$, diferenciável, em que $\mathbb{C} \cong \mathbb{R}^{2}$ e são fixadas bases canônicas no domínio e na imagem. Temos $u_{x}-v_{y}=0$ e $u_{y}+v_{x}=0$. Denotando $u^{1}=u, u^{2}=v, x^{1}=x$ e $x^{2}=y$, segue $B_{1}^{11}=1, B_{1}^{12}=0$, $B_{1}^{21}=0, B_{1}^{22}=1, B_{2}^{11}=0, B_{2}^{12}=-1, B_{2}^{21}=1$ e $B_{2}^{22}=0$.

Para colocar a EDP $B_{a}^{r i} \frac{\partial u^{a}}{\partial x^{i}}=$ numa forma livre de coordenadas, vamos definir a seguinte aplicação: $\gamma_{f}: V \rightarrow W \otimes V^{*}$, onde

$$
\gamma_{f}(x)=\frac{\partial f^{a}(x)}{\partial x^{i}} w_{a} \otimes v^{i}
$$

Em que $\left(v^{1}, \cdots, v^{n}\right)$ é base dual de $\left(v_{1}, \cdots, v_{n}\right)$. Considere $B=\operatorname{span}\left\{B_{a}^{r i} w_{a} \otimes v^{i}: 1 \leq\right.$ $r \leq R\}$, claramente $B$ não depende das bases escolhidas, e $f$ é solução da EDP codificada em $B$ se e somente se $\left\langle b, \gamma_{f}(x)\right\rangle=0, \forall x \in V, \forall b \in B$, onde o produto interno em $W \otimes V^{*}$ é o induzido pelos produtos internos em $V$ e $W$. 
Dizemos que $B$ é o espaços das relações de símbolos. O ortogonal de $B$ é pensado como sendo o espaço das possíveis primeiras derivadas. Precisamente:

Definição 3.1.2. Um tableau é um subespaço linear $A \leq W \otimes V^{*}$. Note que um tableau define uma EDP de primeira ordem, homogênea, com coeficientes constantes, para aplicações de $f: V \rightarrow W$, pela inclusão $\gamma_{f}(V) \subset A$.

Exemplo 3.1.3. Para $f=(u, v): \mathbb{R}^{2} \rightarrow \mathbb{R}^{2}$, e a equação $u_{x}+v_{y}=0$, o espaço de relações de símbolos é $B=\operatorname{span}\left\{e_{1} \otimes d x+e_{2} \otimes d y\right\}$, com tableau $A=B^{\perp}=\operatorname{span}\left\{e_{1} \otimes d x-e_{2} \otimes\right.$ $\left.d y, e_{1} \otimes d y, e_{2} \otimes d x\right\}$. Identificando $\operatorname{Hom}\left(\mathbb{R}^{2},\left(\mathbb{R}^{2}\right)^{*}\right) \operatorname{com} M_{2}(\mathbb{R})$ temos

$$
A=\left\{\left(\begin{array}{cc}
a & c \\
b & -a
\end{array}\right): a, b, c \in \mathbb{R}\right\}
$$

Analogamente ao caso anterior, a equação de Cauchy-Riemann é codificada no tableau $A=\operatorname{span}\left\{a\left(e_{1} \otimes d x+e_{2} \otimes d y\right)+b\left(-e_{2} \otimes d x+e_{1} \otimes d y\right): a, b \in \mathbb{R}\right\}$. Em termos de matrizes:

$$
A=\left\{\left(\begin{array}{cc}
a & -b \\
b & a
\end{array}\right): a, b \in \mathbb{R}\right\} .
$$

Exemplo 3.1.4. Seja $L^{*} \leq V^{*}$ de dimensão $k$, e considere $A=W \otimes L^{*}$. Se $L^{*}=$ $\operatorname{span}\left\{v^{1}, \cdots, v^{k}\right\}$, identificando $W \otimes V^{*}$ com um espaço de matrizes, um elemento em $A$ é da forma $\left(\begin{array}{ll}A_{s \times k} & 0_{s \times(n-k)}\end{array}\right)$, isto é, as equações codificadas por $A$ são $u_{x^{\rho}}^{a}=0$, para $\rho \in\{k+1, \cdots, n\}$ e $a \in\{1, \cdots, s\}$. As soluções são $u^{a}\left(x^{1}, \cdots, x^{n}\right)=f^{a}\left(x^{1}, \cdots, x^{k}\right)$, em que $f^{a}$ são funções analíticas arbitrárias.

Analogamente ao exemplo anterior, se $Y \leq W, \operatorname{dim}(Y)=p$ e $Y=\operatorname{span}\left\{w_{1}, \cdots, w_{p}\right\}$, temos $A=\left(\begin{array}{c}A_{p \times n} \\ 0_{(s-p) \times n}\end{array}\right)$, com equações associadas $u_{x^{i}}^{a}=0$, em que $a \in\{p+1, \cdots, s\}$ e $i \in\{1, \cdots, n\}$. As soluções são $u^{b}\left(x^{1}, \cdots, x^{n}\right)=f^{b}\left(x^{1}, \cdots, x^{n}\right)$ e $u^{a}\left(x^{1}, \cdots, x^{n}\right)=f_{0}^{a}$, para $b \in\{1, \cdots, p\}, a \in\{p+1, \cdots, s\}, f^{b}$ funções arbitrárias e $f_{0}^{a}$ constantes.

Fixemos um tableau $A$. Se uma solução $u$ da EDP associada a $A$ é dada em série de potências, isto é, $u^{a}(x)=p^{a}+p_{i}^{a} x^{i}+p_{i j}^{a} x^{i} x^{j}+\cdots$, então $p_{i_{1} \cdots i_{k}}^{a} w_{a} \otimes v^{i^{1}} \otimes \cdots \otimes v^{i^{k}} \in$ $\left(A \otimes V^{\otimes(k-1)}\right) \cap\left(W \otimes S^{(k)}\left(V^{*}\right)\right)$. De fato, se $u^{a}$ é solução, então $u_{x^{i^{1}} \cdots x^{i^{k-1}}}^{a}$ também são, pois $A$ está associada a uma EDP homogênea e com coeficientes constantes, portanto $B_{a}^{r i^{k}} u_{x^{i^{1} \ldots x^{i^{k}}}}^{a}=0$, ou seja, $B_{a}^{r i^{k}}(k p_{i^{1} \ldots i^{k}}^{a}+\overbrace{\ldots}^{\text {não constantes }})=0, \operatorname{logo} B_{a}^{r i^{k}} p_{i^{1} \ldots i^{k}}^{a}=0$, isto é, $v^{a}\left(x^{1}, \cdots, x^{n}\right)=p_{i^{1} \ldots i^{k}}^{a} x^{i^{k}}$ é também solução, logo $\gamma_{v^{a}}(x) \in A$, isto é, $v_{x^{i}}^{a} w_{a} \otimes v^{i^{k}} \in A$, e 
como $p_{i^{1 \ldots i^{k}}}^{a}$ é coeficiente da solução diferenciável $u^{a}$, portanto simétrica nos índices, temos $p_{i^{1} \ldots i^{k}}^{a} w_{a} \otimes v^{i^{1}} \otimes \cdots \otimes v^{i^{k}} \in W \otimes S^{(k)}\left(V^{*}\right)$ conforme o desejado.

Definição 3.1.5. No contexto anterior, $A^{(l)}:=\left(A \otimes V^{* \otimes l}\right) \cap\left(W \otimes S^{(l+1)} V^{*}\right)$ é dito $l$-ésimo prolongamento de $A$.

Definimos um $p$-tableau por $A \leq W \otimes S^{p} V^{*}$, Analogamente ao caso do tableau, $A$ define uma EDP de ordem $p$, homogênea, com coeficiente constantes e com valores em $W$. Podemos também definir o prolongamento de um p-tableau, por $A^{(l)}=\left(A \otimes V^{* \otimes l}\right) \cap(W \otimes$ $\left.S^{(p+l)} V^{*}\right)$.

Exemplo 3.1.6. Considere a equação $u_{x x}+v_{y y}=0$, para funções $f=(u, v): \mathbb{R}^{2} \rightarrow \mathbb{R}^{2}$, fixadas bases canônicas no domínio e na imagem. Denote $p_{1}^{1}=u_{x}, p_{2}^{1}=u_{y}, p_{1}^{2}=v_{x} \mathrm{e}$ $p_{2}^{2}=v_{y}$. Então o tableau de ordem 2 é dado por: $A=\left\{p_{11}^{1} e_{1} \otimes e_{1} e_{1}+p_{12}^{1} e_{1} \otimes e_{1} e_{2}+\right.$ $\left.p_{22}^{1} e_{1} \otimes e_{2} e_{2}+p_{11}^{2} e_{2} \otimes e_{1} e_{1}+p_{12}^{2} e_{2} \otimes e_{1} e_{2}+p_{22}^{2} e_{2} \otimes e_{2} e_{2}: p_{11}^{1}+p_{22}^{2}=0\right\}$ e $\operatorname{dim}(A)=5$

Até agora, resumidamente, traduzimos (ou codificamos) a definição de uma EDP homogênea e com coeficientes constantes para subespaços lineares. No que segue, apresentaremos uma condição suficiente à existência de uma solução local na linguagem aqui exposta.

Seja $A \leq W \otimes V^{*}$. Fixe $b=\left(v^{1}, \cdots, v^{n}\right)$ e $q=\left(w_{1}, \cdots, w_{n}\right)$ bases ordenadas de $V^{*}$ e $W$, respectivamente. Considere $F_{j}:=\operatorname{span}\left\{v^{j+1}, \cdots v^{n}\right\}, F_{j}^{c}=\operatorname{span}\left\{v^{1}, \cdots, v^{j}\right\}$ e a aplicação linear $\left.i d_{W} \otimes \pi_{j}\right|_{A}: A \rightarrow W \otimes F_{j}^{c},\left.i d_{W} \otimes \pi_{j}\right|_{A}\left(a_{i}^{c} w_{c} \otimes v^{i}\right)=\sum_{c=1}^{s} \sum_{i=1}^{j} a_{i}^{c} w_{c} \otimes v^{j}$. Denotemos $s_{1}(b, q)=\operatorname{dim}\left(\operatorname{Im}\left(\left.i d_{W} \otimes \pi_{1}\right|_{A}\right)\right)$ e, por recursão, $s_{j}(b, q)=\operatorname{dim}\left(\operatorname{Im}\left(\left.d_{W} \otimes \pi_{j}\right|_{A}\right)\right)-\sum_{i=1}^{j-1} s_{i}(b, q)$.

Proposição 3.1.7. No contexto acima, $s_{j}(b, q)$ não depende da base $q$ e

$$
\operatorname{ker}\left(\left.i d_{W} \otimes \pi_{j}\right|_{A}\right)=\left(W \otimes F_{j}\right) \cap A
$$

Demonstração. Do fato de que estão fixadas bases, podemos identificar $A$ com um subespaço de matrizes. Seja $X \in A$. Se $p$ é outra base ordenada de $W$, então $X=M \tilde{X}, M$ matriz de mudança de base. Como $M$ é invertível, segue que a dimensão da imagem de $\left.i d_{W} \otimes \pi_{j}\right|_{A}$ é invariante por $M, \operatorname{logo} s_{j}(b, q)=s_{j}(b, p)$.

Para a igualdade: $\left.i d_{W} \otimes \pi_{j}\right|_{A}\left(a_{i}^{c} w_{c} \otimes v^{i}\right)=0$ que implica em $a_{i}^{c}=0$, para cada $c=1, \cdots, s$ e $i \leq j$, portanto $a_{i}^{c} w_{c} \otimes v^{i} \in W \otimes F_{j}$ a reciproca é imediata. 
Denote $A_{j}(b):=\left(W \otimes F_{j}\right) \cap A$. Pelo teorema do núcleo e da imagem, e da definição de $s_{j}(b, q)=s_{j}(b)$, temos $\operatorname{dim}(A)=\operatorname{dim}\left(A_{j}\right)+\sum_{i=1}^{j} s_{i}(b)$, como $s_{n}(b)=\operatorname{dim}(A)-\sum_{i=1}^{n-1} s_{i}(b)$, segue: $\operatorname{dim}\left(A_{j}(b)\right)=\sum_{i=j+1}^{n} s_{i}(b)$.

Definição 3.1.8. Seja $A \leq W \otimes V^{*}$ um tableau. Definimos: $s_{1}(A)=\max \left\{s_{1}(b): b\right.$ base $\}$ e $s_{j}(A)=\max \left\{s_{j}(b):\right.$ b base, $\left.s_{1}(A)=s_{1}(b), \cdots, s_{j-1}(A)=s_{j-1}(b)\right\}$. Dizemos que $s_{i}(A)$ é o caracter de $A$ e que $b$ é uma base $A$-genérica se $s_{i}(b)=s_{i}(A), \forall i \in\{1, \cdots, n\}$.

Observação 3.1.9. Para os caracteres de $A$ vale: $s \geq s_{1} \geq \cdots \geq s_{n} \geq 0$. Por contradição, suponha $s_{j-1}<s_{j}$. Tome $b=\left(v^{1}, \cdots, v^{n}\right)$ base $A$-genérica, considere $\tilde{b}=\left(v^{1}, \cdots, v^{j}, v^{j-1}, \cdots, v^{n}\right)$. Para $\tilde{b}$ temos $s_{j-1}(b)<s_{j-1}(\tilde{b})$, contradição a maximalidade de $s_{j-1}(A)$.

Exemplo 3.1.10. Sejam $V=\mathbb{R}^{3}, W=\mathbb{R}^{s}$ e

$$
A=\left\{\left(\begin{array}{ccc}
p_{1}^{\lambda} & p_{2}^{\lambda} & 0 \\
p_{1}^{\xi} & 0 & 0
\end{array}\right)_{s \times 3}: p_{i}^{\lambda}, p_{1}^{\xi} \in \mathbb{R}, i \in\{1,2\}, \lambda \in\{1, \cdots, k\}, \xi \in\{k+1, \cdots, s\}\right\} .
$$

A está associado a $\left\{\begin{array}{c}u_{x_{2}}^{\xi}=0 \\ u_{x_{3}}^{a}=0\end{array}\right.$, cujas soluções são da forma $u^{\lambda}=f^{\lambda}\left(x^{1}, x^{2}\right)$ e $u^{\xi}=f^{\xi}\left(x^{1}\right)$. Claramente $s_{1}=s, s_{2}=k$ e $s_{3}=0$. Como $A$ é o espaço das possíveis primeiras derivadas, $A^{(1)}$ é o espaço das possíveis segundas derivadas. Calculando $A^{(1)}$ no nosso caso: $A^{(1)}=\left\{p_{11}^{1} e_{1} \otimes e_{1} e_{1}+p_{12}^{1} e_{1} \otimes e_{1} e_{2}+p_{22}^{1} e_{1} \otimes e_{2} e_{2}+p_{11}^{2} e_{2} \otimes e_{1} e_{1}+p_{12}^{2} e_{2} \otimes e_{1} e_{2}+\right.$ $\left.p_{22}^{2} e_{2} \otimes e_{2} e_{2}+\cdots+p_{22}^{k} e_{k} \otimes e_{2} e_{2}: p_{12}^{\xi}=0, p_{22}^{\xi}=0\right\}$, portanto $\operatorname{dim}\left(A^{(1)}\right)=s+2 k$.

Observação 3.1.11. Seja $U \subset W \otimes S^{d}\left(V^{*}\right)$ e denote

$$
U_{k}=U \cap\left(W \otimes S^{d}\left(\operatorname{span}\left\{v^{k+1}, \cdots, v^{n}\right\}\right)\right) .
$$

Da definição de prolongamento, vale $\left(A_{k}\right)^{(1)}=\left(A^{(1)}\right)_{k}$. Assim, no que segue, não há ambiguidade ao escrever $A_{j}^{(l)}$.

Proposição 3.1.12. Seja $A \leq W \otimes V^{*}$ um tableau. Então $\operatorname{dim}\left(A^{(1)}\right) \leq s_{1}+2 s_{2}+\cdots+n s_{n}$.

Demonstração. Começamos com algumas observações. Do fato de que $F_{k+1} \subset F_{k}$, segue $A_{k} \subset A_{k-1}$. Prolongando ambos os lados, $A_{k}^{(1)} \subset A_{k-1}^{(1)}$. Defina $T: A_{k-1}^{(1)} \rightarrow A_{k-1}$ da 
seguinte maneira: $p=\sum_{a=1}^{s} \sum_{k \leq i<j}^{n} p_{i j}^{a} w_{a} \otimes v^{i} v^{j} \in A_{k-1}^{(1)}$ e $\left.T(p)=v_{k}\right\lrcorner p . T$ está bem definida, é linear e $\operatorname{ker}(T)=A_{k}^{(1)}$. De fato,

$$
\begin{aligned}
\left.v_{k}\right\lrcorner p & =\sum_{a=1}^{s} \sum_{i, j=k}^{n} p_{i j}^{a} w_{a} \otimes\left(\delta_{i k} v^{j}+\delta_{j k} v^{i}\right) \\
& =\sum_{a=1}^{s}\left(\sum_{j=k}^{n} p_{k j}^{a} w_{a} \otimes v^{j}+\sum_{i=k}^{n} p_{i k}^{a} w_{a} \otimes v^{i}\right) \\
& =2 \sum_{a=1}^{s} \sum_{i=k}^{n} p_{i k}^{a} w_{a} \otimes v^{i} \in A_{k-1},
\end{aligned}
$$

portanto está bem definida; e a linearidade segue da definição de produto interior. Resta mostrar que $\operatorname{ker}(T)=A_{k}^{(1)}$. Seja $q=\sum_{a=1}^{s} \sum_{i, j=k+1}^{n} q_{i j}^{a} w_{a} \otimes v^{i} v^{j} \in A_{k}^{(1)}$. Então

$$
\left.v_{k}\right\lrcorner q=\sum_{a=1}^{s}\left(\sum_{i, j=k+1}^{n} q_{i j}^{a} w_{a} \otimes\left(\delta_{i k} v^{j}+\delta_{j k} v^{i}\right)\right)=0,
$$

$\operatorname{logo} A_{k}^{(1)} \subset \operatorname{ker}(T)$. Reciprocamente, $p \in \operatorname{ker}(T)$ nos dá $p_{i k}^{a}=0$, para cada $a \in\{1, \cdots, s\}$ e $i \in\{k, \cdots, n\}$, portanto $p=\sum_{a=1}^{s} \sum_{i, j=k+1}^{n} p_{i j}^{a} w_{a} \otimes v^{i} v^{j} \in A_{k}^{(1)}$.

Das considerações anteriores temos que a seguinte sequência é exata:

$$
0 \longrightarrow A_{k}^{(1)} \stackrel{i}{\longrightarrow} A_{k-1}^{(1)} \stackrel{T}{\longrightarrow} A_{k-1} \text {. }
$$

Agora

$$
\operatorname{dim}\left(A_{k-1}^{(1)}\right)=\operatorname{dim}(\operatorname{ker}(T))+\operatorname{dim}(\operatorname{Im}(T)),
$$

$\mathrm{e} \operatorname{Im}(T) \leq A_{k-1}, \log \mathrm{O}$

$$
\operatorname{dim}(\operatorname{Im}(T)) \leq \operatorname{dim}\left(A_{k-1}\right)
$$

e $\operatorname{dim}\left(A_{k-1}^{(1)}\right) \leq \operatorname{dim}(\operatorname{ker}(T))+\operatorname{dim}\left(A_{k-1}\right)$. Usando que a sequência acima é exata,

$$
\operatorname{dim}\left(A_{k-1}^{(1)}\right) \leq \operatorname{dim}\left(A_{k}^{(1)}\right)+\operatorname{dim}\left(A_{k-1}\right)
$$

isto é,

$$
\operatorname{dim}\left(A_{k-1}^{(1)}\right)-\operatorname{dim}\left(A_{k}^{(1)}\right) \leq \operatorname{dim}\left(A_{k-1}\right)
$$

Somando em $k, \operatorname{dim}\left(A^{(1)}\right)-\operatorname{dim}\left(A_{n}^{(1)}\right) \leq \operatorname{dim}(A)+\operatorname{dim}\left(A_{1}\right)+\cdots+\operatorname{dim}\left(A_{n-1}\right) . A_{n}^{(1)}=0$, uma vez que $F_{n}=\{0\}$, e $\operatorname{dim}(A)=\sum_{i=1}^{s} s_{i}, \operatorname{dim}\left(A_{1}\right)=\sum_{i=2}^{s} s_{i}, \cdots, \operatorname{dim}\left(A_{n-1}\right)=s_{n}$, portanto $\operatorname{dim}\left(A^{(1)}\right) \leq s_{1}+2 s_{2}+\cdots+n s_{n}$

Definição 3.1.13. Um tableau $A$ é involutivo se a desigualdade da Proposição 3.1.12 é na verdade uma igualdade. 
Definição 3.1.14. No mesmo contexto, seja $\sigma \in\{1, \cdots, s\}$. Definimos:

$$
\operatorname{level}(\sigma)= \begin{cases}\max \left\{t: \sigma \leq s_{t}\right\}, & \text { se } \sigma \leq s_{1} \\ 0, & \text { se } \sigma>s_{1}\end{cases}
$$

Exemplo 3.1.15. No exemplo 3.1.10, temos

$$
\operatorname{level}(\sigma)= \begin{cases}2, & \text { se } \sigma \in\{1, \cdots, k\} \\ 1, & \text { se } \sigma \in\{k+1, \cdots, s\},\end{cases}
$$

e $\operatorname{dim}\left(A^{(1)}\right)=s+2 k=s_{1}+2 s_{2}$, logo ele é involutivo.

Estamos prontos para enunciar o que é resolver o tableau.

Teorema 3.1.16. (Cartan-Kähler para tableux). Seja $A \leq W \otimes V^{*}$ um tableau. Sejam uma $\left\{v^{1}, \cdots, v^{n}\right\}$ base A-genérica e $\left\{w_{1}, \cdots, w_{s}\right\}$ base de $W$, com $\left(x^{i}\right)$ e $\left(u^{a}\right)$ as coordenadas respectivamente. Se $A$ é involutiva, então o problema $\gamma_{u}(V) \subset A$, com extensão constante

$$
u^{\sigma}\left(x^{1}, \cdots, x^{k}, 0, \cdots, 0\right)=f^{\sigma}\left(x^{1}, \cdots, x^{k}\right),
$$

para $f^{\sigma}$ funções analíticas dadas, em que level $(\sigma)=k$, tem solução, e essa é única.

Discutiremos a prova do teorema acima nas próximas seções.

Definição 3.1.17. Seja $A$ é involutivo, o número $s_{l} \neq 0$ tal que $s_{l+1}=0$ é dito caracter de $A$ e $l$ é chamado de inteiro de Cartan. Do Teorema 3.1.16, uma solução de $A$ fica determinada dando como condição inicial $s_{l}$ funções de $l$ variáveis, $s_{l-1}-s_{l}$ funções de $l-1$ variáveis, $\cdots, s_{1}-s_{2}$ funções de 1 variável e $s-s_{1}$ constantes.

\subsection{Cartan-Kähler para sistemas Pfaffian lineares}

A finalidade dessa seção é aprofundar o conceito de sistemas diferenciais exteriores, generalizar o Teorema de Cartan-Kähler para tableaux e apresentar algumas aplicações à geometria Riemanniana.

Definição 3.2.1. Sejam $\Sigma$ variedade diferencial e $\theta^{1}, \cdots, \theta^{s} \in \Omega^{1}(\Sigma)$ linearmente independentes, e defina $I:=\left\{\theta^{a}\right\}_{\text {alg. Seja } \Omega} \Omega \omega^{1} \wedge \cdots \wedge \omega^{n}, \Omega \neq 0$ representa uma condição de independência, em que $\omega^{i} \in \Omega^{1}(\Sigma)$ e $i \in\{1, \cdots, n\}$. Seja $J=\left\{\omega^{i}\right\}_{\text {alg }}$ e considere 
o ideal $\mathcal{I}=\left\{\theta^{a}\right\}_{\text {diff }}$. No que segue, está implícita em $J$ a condição de independência e escreveremos $(I, J)$ ao invés de $(\mathcal{I}, \Omega)$. Dizemos que $(I, J)$ é um sistema linear de Pfaff se $d \theta^{a} \equiv 0 \bmod J$, para $a \in\{1, \cdots, s\}$.

Proposição 3.2.2. Seja $(I, J)$ um sistema linear de Pfaff. Sejam $\pi^{\epsilon} \in \Omega^{1}(\Sigma), 1 \leq \epsilon \leq$ $\operatorname{dim}(\Sigma)-n-s$ tais que $\left\{\theta^{a}, \omega^{i}, \pi^{\epsilon}\right\}$ é correferencial. Então existe funções locais $A_{\epsilon i}^{a}$ e $T_{i j}^{a}$ satisfazendo

$$
d \theta^{a} \equiv A_{\epsilon i}^{a} \pi^{\epsilon} \wedge \omega^{i}+\frac{1}{2} T_{i j}^{a} \omega^{i} \wedge \omega^{j} \bmod I
$$

Demonstração. Como $\left\{\theta^{a}, \omega^{i}, \pi^{\epsilon}\right\}$ é correferencial, existem funções $B_{b c}^{a}, C_{b i}^{a}, D_{\epsilon b}^{a}, \frac{1}{2} T_{i j}^{a}$, $E_{\epsilon i}^{a}, A_{\epsilon i}^{a}$ e $F_{\epsilon \delta}^{a}$, com $B_{b c}^{a}, \frac{1}{2} T_{i j}^{a}$ e $F_{\epsilon \delta}^{a}$ simétricas nos índices inferiores, tais que

$$
\begin{aligned}
d \theta^{a} & =\underbrace{B_{b c}^{a} \theta^{b} \wedge \theta^{c}}_{0 \text { mod I }}+\underbrace{C_{b i}^{a} \theta^{b} \wedge \omega^{i}}_{0 \text { mod I }}+\underbrace{D_{\epsilon b}^{a} \pi^{\epsilon} \wedge \theta^{b}}_{0 \text { mod I }} \\
& +\frac{1}{2} T_{i j}^{a} \omega^{i} \wedge \omega^{j}+A_{\epsilon i}^{a} \pi^{\epsilon} \wedge \omega^{i}+F_{\epsilon \delta}^{a} \pi^{\epsilon} \wedge \pi^{\delta} \\
& \equiv A_{\epsilon i}^{a} \pi^{\epsilon} \wedge \omega^{i}+\frac{1}{2} T_{i j}^{a} \omega^{i} \wedge \omega^{j}+F_{\epsilon \delta}^{a} \pi^{\epsilon} \wedge \pi^{\delta} \bmod I .
\end{aligned}
$$

Do fato de que $d \theta^{a} \equiv 0 \bmod J$, já que $(I, J)$ é Pfaffian linear, segue $F_{\epsilon \delta}^{a}=0$, portanto temos (3.1).

Exemplo 3.2.3. Seja $\Sigma \subset J^{k}(M, N)$ subvariedade, e considere o sistema de contato canônico em $\Sigma$. Temos $I=\left\{\theta^{a}=d u^{a}-p_{i}^{a} d x^{i}, \theta_{I}^{a}=d p_{I}^{a}-p_{I j}^{a} d x^{j}\right\}$ e $J=\left\{\theta^{a}, \theta_{I}^{a}, d x^{i}\right\}$. O cálculo $d I \bmod J$ é dado por $d \theta^{a}$ e $d \theta_{I}^{a} \bmod J$, isto é $d \theta^{a}=-d p_{j}^{a} \wedge d x^{i} \equiv 0 \bmod J$ e $d \theta_{I}^{a}=d p_{I j}^{a} \wedge d x^{j} \equiv 0 \bmod J$, portanto o sistema de contato canônico é Pfafian linear.

Exemplo 3.2.4. Sejam $\Sigma=A S O(n+s)$ e $\omega$ forma de Maurer-Cartan. Defina:

$$
I=\left\{\omega^{n+1}, \cdots, \omega^{n+s}\right\} \text { e } J=\left\{\omega^{1}, \cdots \omega^{n}, \omega^{n+1}, \cdots, \omega^{n+s}\right\}
$$

Do fato de que $d \omega=-\omega \wedge \omega$, segue $d \omega^{a}=-\omega_{i}^{a} \wedge \omega^{i}-\omega_{b}^{a} \wedge \omega^{b} \equiv 0 \bmod J$, portanto Pfaffian linear.

Seja $\Sigma=A S O(n+s) \times \mathbb{R}^{s\left(\begin{array}{c}n+1 \\ 2\end{array}\right)}$, com $h_{i j}^{a}$ coordenadas no segundo termo, essas simétricas nos índices inferiores. Defina $I=\left\{\omega^{a}, \omega_{j}^{a}-h_{j k}^{a} \omega^{k}\right\}, J=\left\{\omega^{j}, \omega^{a}, \omega_{j}^{a}-h_{j k}^{a} \omega^{k}\right\}$, em que $a \in\{n+1, \cdots, n+s\}$ e $j, k \in\{1, \cdots, n\}$. Do exemplo anterior $d \omega^{a} \equiv 0 \bmod J$. Resta 
calcular

$$
\begin{aligned}
d\left(\omega_{j}^{a}-h_{j k}^{a} \omega^{k}\right) & =d \omega_{j}^{a}-\underbrace{d h_{j k}^{a} \wedge \omega^{k}}_{0 \bmod J}-h_{j k}^{a} d \omega^{k} \\
& \equiv-\omega_{i}^{a} \wedge \omega_{j}^{i}-\omega_{b}^{a} \wedge \omega_{j}^{b}+h_{j k}^{a}\left(\omega_{i}^{k} \wedge \omega^{i}+\omega_{a}^{k} \wedge \omega^{a}\right) \bmod J \\
& \equiv-\omega_{i}^{a} \wedge \omega_{j}^{i}-\omega_{b}^{a} \wedge \omega_{j}^{b}+\underbrace{h_{j k}^{a} \omega_{i}^{k} \wedge \omega^{i}}_{0 \bmod J}+\underbrace{h_{j k}^{a} \omega_{a}^{k} \wedge \omega^{a}}_{0 \bmod J} \bmod J \\
& \equiv-\omega_{i}^{a} \wedge \omega_{j}^{i}-\omega_{b}^{a} \wedge \omega_{j}^{b} \bmod J
\end{aligned}
$$

Note que $\omega_{i}^{a}=\underbrace{\omega_{i}^{a}-h_{i j}^{a} \omega^{j}}_{\in J}+\underbrace{h_{i j}^{a} \omega^{j}}_{\in J}$, portanto $\omega_{i}^{a} \wedge \star \equiv 0 \bmod J$, segue que $d I \equiv 0 \bmod J$ e $(I, J)$ é Pfaffian linear.

Observação 3.2.5. No exemplo anterior, as variedades integrais do primeiro caso são levantamentos para $A S O(n+s)$ de subvariedades de dimensão $n$ de $\mathbb{E}^{n+s}$, já no segundo caso são os levantamentos das subvariedades de dimensão $n$ de $\mathbb{E}^{n+s}$ munidas da sua segunda forma fundamental.

Exemplo 3.2.6. Sejam $\Sigma=\mathbb{R} \times \mathbb{R}^{5}$, com coordenadas $\left(x, y^{i}\right), I=\{\theta\}$ e $J=\{\theta, d x\}$, em que $\theta=y^{1} d y^{2}+y^{3} d y^{4}+y^{5} d x$. Vamos verificar que $(I, J)$ não é Pfaffian linear. Para pontos em que $y^{1} \neq 0$, temos $d y^{2}=\frac{\theta-y^{3} d y^{4}-y^{5} d x}{y^{1}}$ e

$$
\begin{aligned}
d \theta & =d y^{1} \wedge d y^{2}+d y^{3} \wedge d y^{4}+d y^{5} \wedge d x \\
& =d y^{1} \wedge\left(\frac{\theta-y^{3} d y^{4}-y^{5} d x}{y^{1}}\right)+d y^{3} \wedge d y^{4}+d y^{5} \wedge d x \\
& =\underbrace{d y^{1} \wedge \frac{\theta}{y^{1}}}_{0 \bmod J}-\frac{y^{3}}{y^{1}} d y^{1} \wedge d y^{4}-\underbrace{\frac{y^{5}}{y^{1}} d y^{1} \wedge d x}_{0 \bmod J}+d y^{3} \wedge d y^{4}+\underbrace{d y^{5} \wedge d x}_{0 \bmod J} \\
& \equiv\left(d y^{3}-\frac{y^{3}}{y^{1}} d y^{1}\right) \wedge d y^{4} \bmod J .
\end{aligned}
$$

Segue que $(I, J)$ não é Pfaffian linear.

Observação 3.2.7. No que segue vamos construir o algoritmo de Cartan via exemplos, ou seja, vamos analisar casos particulares e coletar os aspectos gerais com vistas a enunciar a versão do Teorema de Cartan-Kähler para sistemas Pfaffian lineares.

Exemplo 3.2.8. Seja $\Sigma=\mathbb{R}^{3}$ com coordenadas $(x, y, u)$. Sejam $A, B: \mathbb{R}^{3} \rightarrow \mathbb{R}$ funções diferenciáveis, $\theta=d u-A d x-B d y$ e $\Omega=d x \wedge d y$. Temos que uma subvariedade integral 
de $(\{\theta\}, \Omega)$ é o gráfico de uma solução do problema $u_{x}=A$ e $u_{y}=B$. Por outro lado $(\{\theta\}, \Omega)$ é Pfaffian linear pois $J=\{\theta, d x, d y\}$ é correferencial. Aplicando a Proposição 3.2.2, temos $d \theta=\left(A_{y}-B_{x}+A_{u} B-B_{u} A\right) d x \wedge d y \bmod I$, isto é $T=A_{y}-B_{x}+A_{u} B-B_{u} A$, ou seja, as variedades integrais necessariamente estão contidas em $\Sigma^{\prime} \subset \Sigma$ definida por $T=0$. Esse é um caso especial do que chamaremos de torção.

Definição 3.2.9. (Torção aparente local). Seja $T_{i j}^{a}$ como em (3.1). Dizemos que o conjunto formado por essas funções, quando não identicamente nulas, é a torção aparente, denotado $[T]$. Dizemos também que a torção aparente é absorvível se existe uma escolha de complemento $\tilde{\pi}^{\epsilon}$ tal que $\tilde{T}_{i j}^{a}$ são identicamente nulas; caso não exista essa escolha, o conjunto das $T_{i j}^{a}$ é dito simplesmente torção. No exemplo anterior, como $\{\theta, d x, d y\}$ é correferencial, segue que $[T]$ é torção.

Observação 3.2.10. No contexto acima, se $\tilde{\pi}^{\epsilon}=M_{\delta}^{\epsilon} \pi^{\delta}$, para $\left(M_{\delta}^{\epsilon}\right)$ inversível, então

$$
\begin{aligned}
d \theta^{a} & \equiv \tilde{A}_{\epsilon i}^{a} \tilde{\pi}^{\epsilon} \wedge \omega^{i}+\frac{1}{2} \tilde{T}_{i j}^{a} \omega^{i} \wedge \omega^{j} \bmod I \\
& \equiv \tilde{A}_{\epsilon i}^{a} M_{\delta}^{\epsilon} \pi^{\delta} \wedge \omega^{i}+\frac{1}{2} \tilde{T}_{i j}^{a} \omega^{i} \wedge \omega^{j} \bmod I \\
& \equiv \tilde{A}_{\delta i}^{a} M_{\epsilon}^{\delta} \pi^{\epsilon} \wedge \omega^{i}+\frac{1}{2} \tilde{T}_{i j}^{a} \omega^{i} \wedge \omega^{j} \bmod I
\end{aligned}
$$

portanto $A_{\epsilon i}^{a}=\tilde{A}_{\delta i}^{a} M_{\epsilon}^{\delta}$ e $T_{i j}^{a}=\tilde{T}_{i j}^{a}$. Em particular, $\tilde{T}_{i j}^{a}$ é identicamente nula se e somete se $T_{i j}^{a}$ o é. De modo análogo, se $\tilde{\pi}^{\epsilon}=\pi^{\epsilon}+M_{a}^{\epsilon} \theta^{a}$, então $\tilde{T}_{i j}^{a}=T_{i j}^{a}$, já que estamos analisando a classe de $d \theta^{a} \bmod I=\left\{\theta^{1}, \cdots, \theta^{s}\right\}$. Com base no exemplo, a primeira parte do algoritmo de Cartan é determinar a equação para torção via (3.1) e restringir a $\Sigma^{\prime} \subset \Sigma$ onde a torção é nula, esquematizando: 


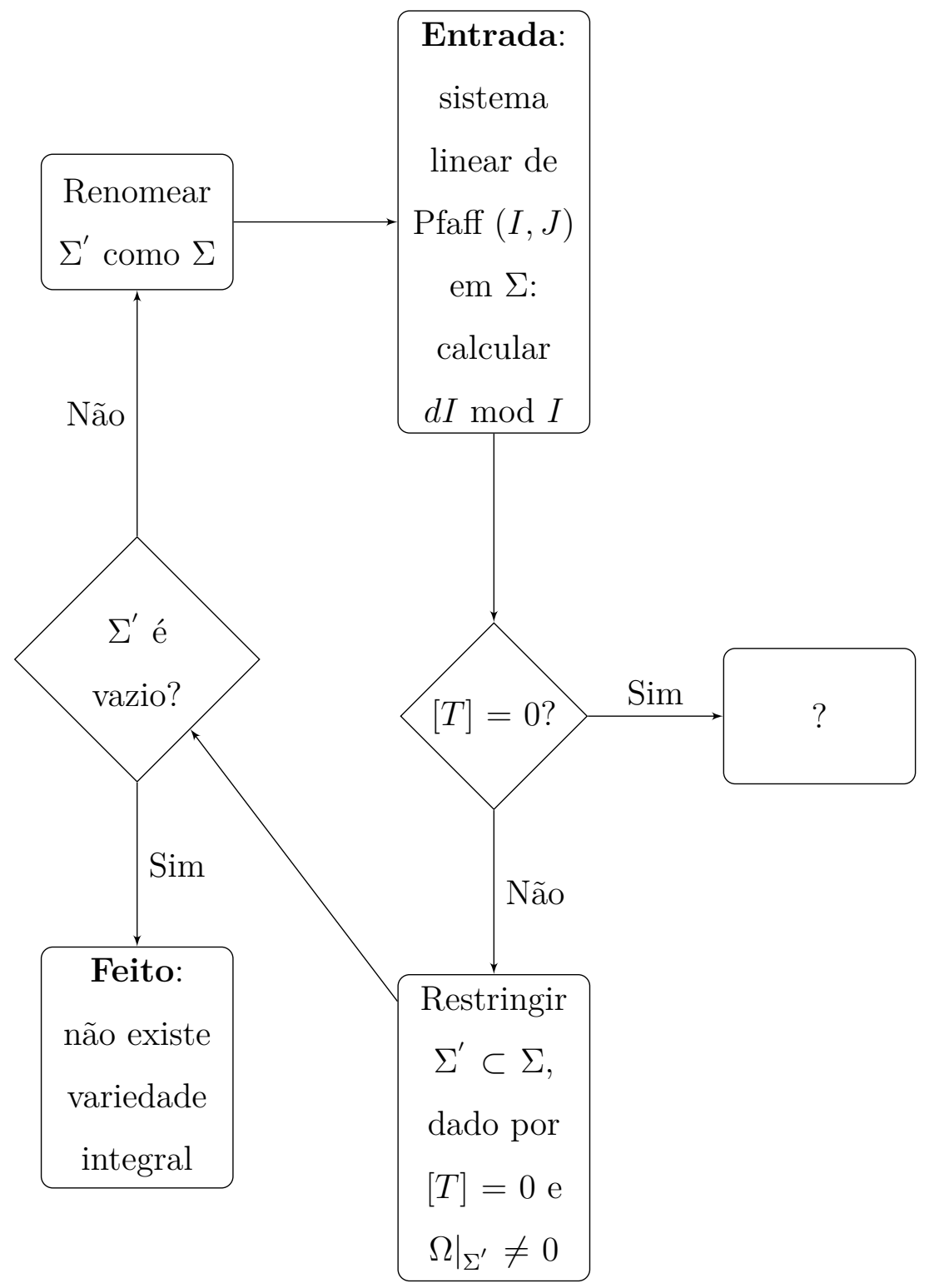

Exemplo 3.2.11. Suponha $i: \Sigma \rightarrow J^{1}(V, W)=V+W+W \otimes V^{*}$ subvariedade definida da seguinte maneira: fixe bases ordenadas $b=\left(v_{1}, \cdots, v_{n}\right)$ e $q=\left(w_{1}, \cdots, w_{s}\right)$ de $V$ e $W$, respectivamente. Seja $F: J^{1}(V, W) \rightarrow \mathbb{R}^{R}, F\left(x^{i}, u^{a}, p_{i}^{a}\right)=\left(B_{a}^{1 i} p_{i}^{a}, \cdots, B_{a}^{R i} p_{i}^{a}\right)$, tal que $F^{-1}(\{0\})=\Sigma$, considere o sistema de contato canônico, neste caso gerado por $\theta^{a}=d u^{a}-p_{i}^{a} d x^{i}$ e $\omega^{i}:=d x^{i}$, em $\Sigma$ e o sistema $(I, J)$, em que $I=\left\{\theta^{a}\right\}$ e $J=\left\{\theta^{a}, \omega^{i}\right\}$. Claramente $d \theta^{a}=-d p_{i}^{a} \wedge \omega^{i}$. A partir de $B_{a}^{r i} p_{i}^{a}=0$, podemos tomar $\pi^{\epsilon}$ tal que $\left\{\theta^{a}, \omega^{i}, \pi^{\epsilon}\right\}$ seja correferencial $\Sigma$ e valha $d p_{i}^{a}=-A_{\epsilon i}^{a} \pi^{\epsilon}$, e do fato que $\Sigma$ é definida por $B_{a}^{r i} p_{i}^{a}=0$, $r \in\{1, \cdots, R\}, B_{a}^{r i} A_{\epsilon i}^{a}=0$ e $d \theta^{a} \equiv A_{\epsilon i}^{a} \pi^{\epsilon} \wedge \omega^{i} \bmod I$. Observe que $(J / I)_{x}=\left\{\omega^{i}+\right.$ $I\}=\left\{d x^{i}+I\right\} \cong V^{*}$ e $I_{x}=\left\{\theta^{a}=d u^{a}-p_{i}^{a} d x^{i}\right\} \cong W^{*}$. Recuperamos o tableau $A=\left\{A_{\epsilon i}^{a} w_{a} \otimes v^{i}: \operatorname{dim}(J)+1 \leq \epsilon \leq \operatorname{dim}(\Sigma)\right\}$. Da seção anterior, temos a existência de solução local se $A$ é involutivo, ampliando nosso algoritmo para: 


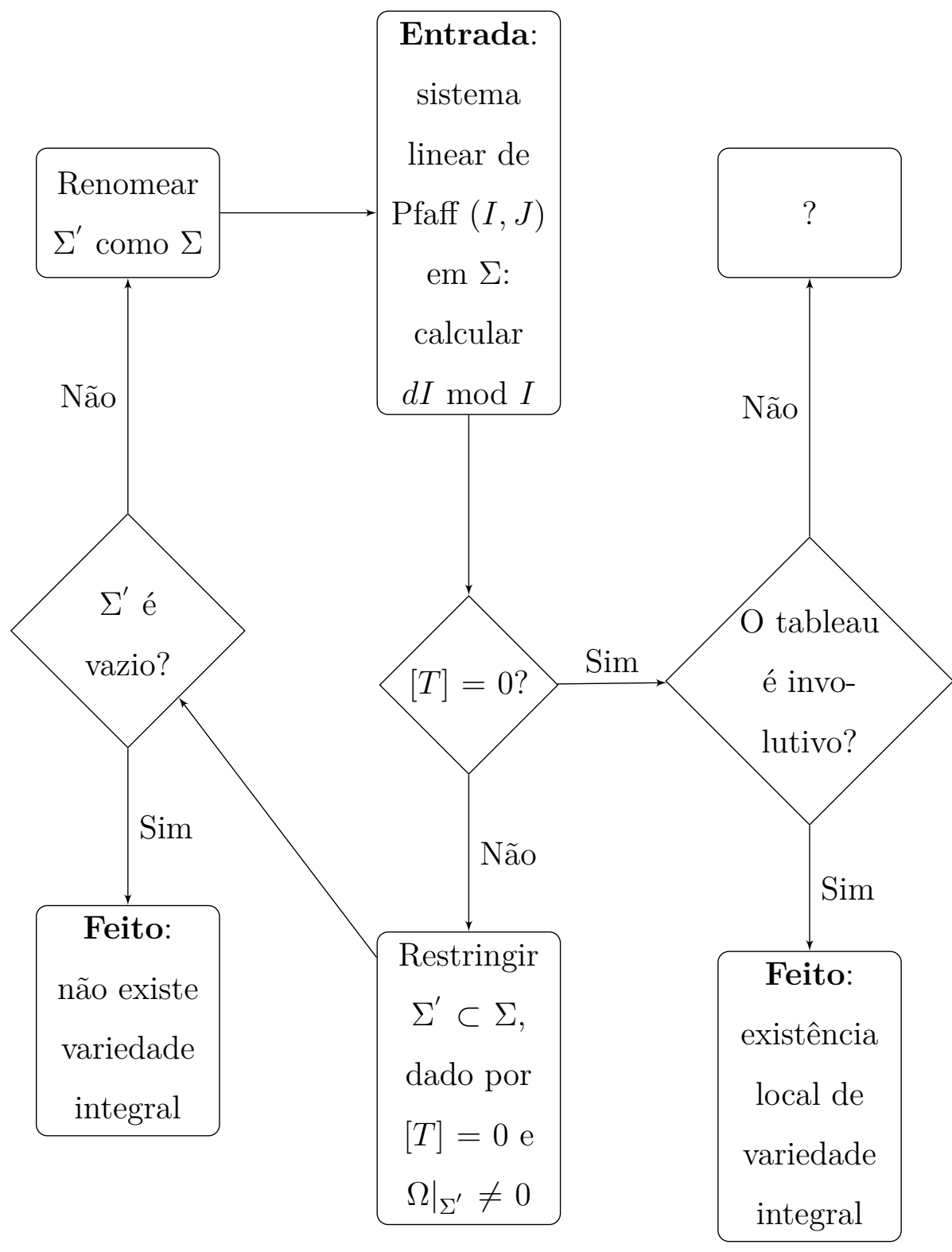

Exemplo 3.2.12. Analogamente ao exemplo anterior, poderíamos considerar uma EDP de primeira ordem e com coeficientes constantes, mas não homogênea, i.e., $F\left(x^{i}, u^{a}, p_{i}^{a}\right)=$ $\left(B_{a}^{1 i} p_{i}^{a}-C_{i}^{1} x^{i}, \cdots, B_{a}^{R i} p_{i}^{a}-C_{i}^{R} x^{i}\right)$. Para esse caso teríamos $d p_{i}^{a}=-A_{\epsilon i}^{a} \pi^{\epsilon}+\frac{1}{2} T_{i j}^{a} \omega^{j} \mathrm{e}$ $d \theta^{a} \equiv A_{\epsilon i}^{a} \pi^{\epsilon} \wedge \omega^{i}+\frac{1}{2} T_{i j}^{a} \omega^{i} \wedge \omega^{j}, \operatorname{com} A_{\epsilon i}^{a} B_{a}^{r i}=0$ e $C_{i}^{r}=B_{a}^{r j} T_{j i}^{a}$ em que $[T]$ pode ser absorvível ou não.

Observação 3.2.13. Nos dois últimos exemplos, se $A$ não é involutivo podemos proceder da seguinte maneira: consideramos $J^{2}(V, W) \cong V+W+W \otimes V^{*}+W \otimes S^{2}\left(V^{*}\right)$, cujas coordenadas são $\left(x^{i}, u^{a}, p_{i}^{a}, p_{i j}^{a}\right)$ e $\tilde{\Sigma}:=V \times W \times A \times A^{(1)}$. O sistema de contato canônico em $\tilde{\Sigma}$ é

$$
\begin{aligned}
& \theta^{a}:=d u^{a}-p_{i}^{a} d x^{i}, \\
& \theta_{i}^{a}:=d p_{i}^{a}-p_{i j}^{a} d x^{j} .
\end{aligned}
$$


Definindo novamente $\omega^{i}=d x^{i}$, temos o sistema $\left(\tilde{I}=\left\{\theta^{a}, \theta_{i}^{a}\right\}, \tilde{J}=\left\{\theta^{a}, \theta_{i}^{a}, \omega^{i}\right\}\right)$. Nesse sistema

$$
\begin{aligned}
d \theta^{a} & =-d p_{i}^{a} \wedge \omega^{i}=-\left(\theta_{i}^{a}+p_{i j}^{a} \omega^{j}\right) \wedge \omega^{i} \\
& =\theta_{i}^{a} \wedge \omega^{i}+p_{i j}^{a} \omega^{j} \wedge \omega^{i} \\
& \equiv p_{i j}^{a} \omega^{j} \wedge \omega^{i} \bmod \widetilde{I}
\end{aligned}
$$

entretanto

$$
p_{i j}^{a} \omega^{j} \wedge \omega^{i}=-p_{i j}^{a} \omega^{i} \wedge \omega^{j}=-p_{j i}^{a} \omega^{i} \wedge \omega^{j} \stackrel{i \leftrightarrow j}{=}-p_{i j}^{a} \omega^{j} \wedge \omega^{i}
$$

$\log 0 p_{i j}^{a} \omega^{j} \wedge \omega^{i}=0$. Assim, em termos de matrizes, um elemento do tableau $\tilde{A}$ é da forma $\left(\begin{array}{c}0_{s \times n} \\ A_{(\operatorname{dim}(A)) \times n}\end{array}\right)$. Se esse novo tableau for involutivo, então existe uma solução local. Caso contrario, prolongamos para $J^{3}(V, W)$ e assim por diante, diagramaticamente: 


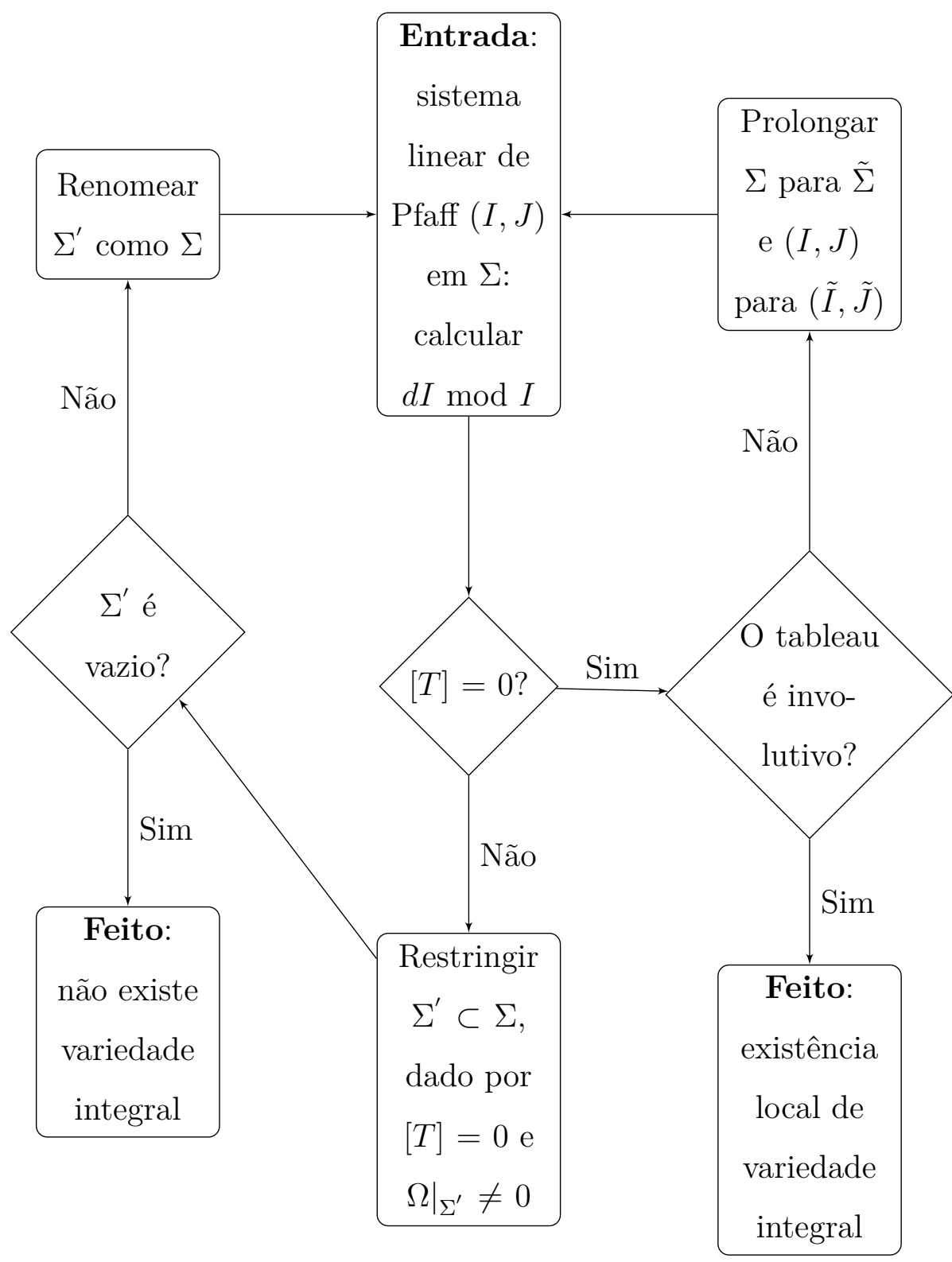

Esboçado o esquema que gostaríamos de seguir para resolver um sistema linear de Pfaff $(I, J)$, vamos as definições precisas.

Definição 3.2.14. Seja $(I, J)$ sistema linear de Pfaff em $\Sigma$. Escolha geradores linearmente independentes de modo que $I=\left\{\theta^{a}\right\}$ e $J=\left\{\omega^{i}\right\}$, e complemento $\left\{\pi^{\epsilon}\right\}$, tais que $\left\{\theta^{a}, \omega^{i}, \pi^{\epsilon}\right\}$ seja um correferencial em $T^{*} \Sigma$, onde $a \in\{1, \cdots, s\}, i \in\{1, \cdots, n\}$ e $\epsilon \in\{1, \cdots, \operatorname{dim}(\Sigma)-$ $\operatorname{dim}(J)\}$. Para cada $x \in \Sigma$, defina $V^{*}:=(J / I)_{x}$ e $W:=I_{x}$, denote $w^{a}=\theta_{x}^{a}$ e $v^{j}=\omega_{x}^{j}$. Então o tableau de $(I, J)$ em $x$ é dado por

$$
A_{x}:=\operatorname{span}\left\{A_{\epsilon i}^{a} w_{a} \otimes v^{i}: \epsilon \in\{1, \cdots, \operatorname{dim}(\Sigma)-\operatorname{dim}(J)\}\right\} \subset W \otimes V^{*}
$$

onde $A_{\epsilon i}^{a}$ é dada por (3.1). Definimos a relação de símbolos $B=\operatorname{span}\left\{B_{a}^{r i} w_{a} \otimes v^{i}: r \in\right.$ $\{1, \cdots, R\}$ em que $B=A^{\perp}$. 
Proposição 3.2.15. Na definição acima, $A_{x}$ não depende das escolhas de geradores $e$ complemento.

Demonstração. Vamos analisar caso a caso,

$1^{\mathrm{o}}$ caso: $\tilde{\theta}^{a}=M_{b}^{a} \theta^{b}$, com $M_{b}^{a}$ funções diferenciáveis e localmente definidas em $\Sigma$, temos:

$$
\begin{aligned}
d \tilde{\theta}^{a} & =\underbrace{d M_{b}^{a} \theta^{b}}_{0 \bmod I}+M_{b}^{a} d \theta^{b} \\
& \equiv M_{b}^{a} A_{\epsilon i}^{b} \pi^{\epsilon} \wedge \omega^{i}+\frac{1}{2} M_{b}^{a} T_{i j}^{b} \omega^{i} \wedge \omega^{j} \bmod I
\end{aligned}
$$

portanto $\tilde{A}_{\epsilon i}^{a}=M_{b}^{a} A_{\epsilon i}^{b}$, assim

$$
\begin{aligned}
\tilde{A}_{x} & =\operatorname{span}\left\{\tilde{A}_{\epsilon i}^{a} \tilde{w}_{a} \otimes v^{i}\right\} \\
& =\operatorname{span}\left\{\sum_{a}\left(M_{b}^{a} A_{\epsilon i}^{b}\right)\left(\sum_{c}\left(M_{a}^{c}\right)^{-1} w_{c}\right) \otimes v^{i}\right\} \\
& =\operatorname{span}\left\{\sum_{a, c} M_{b}^{a}\left(M_{a}^{c}\right)^{-1} A_{\epsilon i}^{b} w_{c} \otimes v^{i}\right\} \\
& =A_{x}
\end{aligned}
$$

$2^{\mathrm{o}}$ caso: $\tilde{\omega}^{i}=M_{j}^{i} \omega^{j}$. De (3.1) temos

$$
d \theta^{a} \equiv \tilde{A}_{\epsilon i}^{a} \pi^{\epsilon} \wedge \tilde{\omega}^{i}+\cdots \bmod I \equiv \tilde{A}_{\epsilon i}^{a} M_{j}^{i} \pi^{\epsilon} \wedge \omega^{j}+\cdots \bmod I
$$

$\operatorname{assim} A_{\epsilon j}^{a}=M_{j}^{i} \tilde{A}_{\epsilon i}^{a}$, portanto

$$
\begin{aligned}
\tilde{A}_{x} & =\operatorname{span}\left\{\tilde{A}_{\epsilon i}^{a} w_{a} \otimes \tilde{v}^{i}\right\} \\
& =\operatorname{span}\left\{\left(M^{-1}\right)_{i}^{j} A_{\epsilon j}^{a} w_{a} \otimes\left(M_{k}^{i} v^{k}\right)\right\} \\
& =\operatorname{span}\left\{\left(M^{-1}\right)_{i}^{j}\left(M_{k}^{i}\right) A_{\epsilon j}^{a} w_{a} \otimes v^{k}\right\} \\
& =\operatorname{span}\left\{\delta_{k}^{j} A_{\epsilon j}^{a} w_{a} \otimes v^{k}\right\} \\
& =\operatorname{span}\left\{A_{\epsilon j}^{a} w_{a} \otimes v^{j}\right\} \\
& =A_{x}
\end{aligned}
$$

$3^{\mathrm{o}}$ caso: $\tilde{\pi}^{\epsilon}=M_{\delta}^{\epsilon} \pi^{\delta}$, análogo ao demais $\operatorname{casos} A_{\epsilon i}^{a}=M_{\epsilon}^{\delta} \tilde{A}_{\delta i}^{a} \mathrm{e}$

$$
A_{x}=\operatorname{span}\left\{A_{\epsilon i}^{a} w_{a} \otimes v^{i}\right\}=\operatorname{span}\left\{M_{\epsilon}^{\delta} \tilde{A}_{\delta i}^{a} w_{a} \otimes v^{i}\right\}=\tilde{A}_{x}
$$


Observação 3.2.16. Definido o tableau de um sistema $(I, J)$ Pfaffian linear qualquer precisamos formalizar os conceitos de torção e prolongamento. Para o primeiro procedemos da seguinte forma: queremos escolher um complemento de forma que a torção seja nula. Uma troca de complemento em termos de álgebra linear básica é $\tilde{\pi}^{\epsilon}=M_{\delta}^{\epsilon} \pi^{\delta}+e_{i}^{\epsilon} \omega^{i}+f_{a}^{\epsilon} \theta^{a}$. Como estamos calculado $d \theta^{a} \bmod I$, podemos supor, sem perca de generalidade, $f_{a}^{\epsilon}=0$. Assim no novo correferencial, $d \theta^{a} \equiv \tilde{A}_{\delta i}^{a} M_{\epsilon}^{\delta} \pi^{\epsilon} \wedge \omega^{i}+\left(\frac{1}{2} \widetilde{T}_{i j}^{a}-e_{j}^{\epsilon} \tilde{A}_{\epsilon i}^{a}\right) \omega^{i} \wedge \omega^{j}$. Do terceiro caso da proposição acima, o tableau é invariante por $\tilde{\pi}^{\epsilon}=M_{\delta}^{\epsilon} \pi^{\delta}$, então podemos supor que $M=I d$, assim $\tilde{A}_{\epsilon i}^{a}=A_{\epsilon i}^{a}$, portanto $d \theta^{a} \equiv A_{\epsilon i}^{a} \pi^{\epsilon} \wedge \omega^{i}+\left(\frac{1}{2} \widetilde{T}_{i j}^{a}-e_{j}^{\epsilon} A_{\epsilon i}^{a}\right) \omega^{i} \wedge \omega^{j}$. Colocando o segundo termo na base, temos $\tilde{T}_{i j}^{a}=T_{i j}^{a}-\underbrace{\left(A_{\epsilon j}^{a} e_{i}^{\epsilon}-A_{\epsilon i}^{a} e_{j}^{\epsilon}\right)}_{:=2 E_{i j}^{a}}$. Considere a aplicação de anti-simetrização $\delta: W \otimes V^{*} \otimes V^{*} \rightarrow W \otimes \Lambda^{2}\left(V^{*}\right)$, dada por $\delta\left(w_{a} \otimes v^{i} \otimes v^{j}\right)=w_{a} \otimes v^{i} \wedge v^{j}$ (e estendida linearmente). Temos que $E_{i j}^{a} w_{a} \otimes v^{i} \wedge v^{j} \in \delta\left(A_{x} \otimes V^{*}\right)$, uma vez que

$$
\begin{aligned}
\delta\left(e_{j}^{\epsilon} A_{\epsilon i}^{a} w_{a} \otimes v^{i} \otimes v^{j}\right) & =e_{j}^{\epsilon} A_{\epsilon i}^{a} w_{a} \otimes v^{i} \wedge v^{j} \\
& =\sum_{i<j} 2 E_{i j}^{a} w_{a} \otimes v^{i} \wedge v^{j} \\
& =E_{i j}^{a} w_{a} \otimes v^{i} \wedge v^{j}
\end{aligned}
$$

logo o espaço das possíveis modificações é $\delta\left(A_{x} \otimes V^{*}\right)$ e temos a

Definição 3.2.17. A torção de $(I, J)$ em $x \in \Sigma$ é a classe $[T]:=\left[T_{i j}^{a} w_{a} \otimes v^{i} \wedge v^{j}\right] \in$ $W \otimes \Lambda^{2}\left(V^{*}\right) / \delta\left(A_{x} \otimes V^{*}\right)$.

Observação 3.2.18. Na definição anterior, se $[T]=0$, então existe $E_{i j}^{a}$ tal que $T_{i j}^{a}+E_{i j}^{a}=$ 0 . Como $E_{i j}^{a} w_{a} \otimes v^{i} \wedge v^{j} \in \delta\left(A_{x} \otimes V^{*}\right)$, existem $e_{i}^{\epsilon}$ tais que $E_{i j}^{a}=A_{\epsilon j}^{a} e_{i}^{\epsilon}-A_{\epsilon i}^{a} e_{j}^{\epsilon}$ e a torção é absorvida com o complemento $\tilde{\pi}^{\epsilon}=\pi^{\epsilon}+e_{i}^{\epsilon} \omega_{i}$.

Resta generalizar o prolongamento de $(I, J)$ :

Definição 3.2.19. Sejam $\mathcal{I}$ um sistema diferencial exterior em $\Sigma$ e $\pi: G_{n}(T \Sigma) \rightarrow \Sigma$ o fibrado Grassmanniano. Para cada ponto $(p, E) \in G_{n}(T \Sigma)$, definimos o $I_{(p, E)}$ como sendo o ideal algébrico gerado por $\pi^{*}\left(E^{\perp}\right)$, e $J_{(p, \Sigma)}$ como sendo o ideal gerado por $\pi^{*}\left(T_{p}^{*} \Sigma\right)$. Analogamente à Definição 1.1.3, temos o conjunto dos elementos integrais $\mathcal{V}_{n}(\mathcal{I}):=\{(p, E) \in$ $\left.G_{n}(T \Sigma):\left.\alpha_{x}\right|_{E}=0, \forall \alpha_{x} \in \mathcal{I}_{x}\right\}$; dizemos que $\left.(I, J)\right|_{\mathcal{V}_{n}(\mathcal{I})}$ é o prolongamento de $\mathcal{I}$.

Munidos da linguagem acima, estamos prontos para enunciar o teorema de CartanKähler para sistemas lineares de Pfaff e o algoritmo geral para garantir existência de variedades integrais desses sistemas. 
Teorema 3.2.20. (Cartan-Kähler para sistema liner de Pfaff. Seja $(I, J)$ um sistema linear de Pfaff em $\Sigma$, e seja $x \in \Sigma$ tal que existe $U$ vizinhança de $x$ satisfazendo:

1. $[T]_{y}=0, \forall y \in U$.

2. $A_{y}$ é involutivo para cada $y \in U$.

Então existe variedade integral de $(I, J)$ passando por $x$.

Observação 3.2.21. No Exemplo 3.1.11 vimos que uma EDP de primeira ordem, homogênea e com coeficientes constantes dada por $F^{-1}(\{0\})$ naturalmente traduz-se para um sistema Pfaffian linear que satisfaz $d \theta^{a}=A_{\epsilon i}^{a} \pi^{\epsilon} \wedge \omega^{i}$, isto é, sem torção. Se $A$ for involutivo, podemos aplicar o teorema de Cartan-Kähler acima e temos que por $x \in \Sigma=F^{-1}(\{0\})$ passa uma subvariedade integral. Pelo Exemplo 1.1.5 segue que as variedades integrais de $(I, J)$ são justamente os levantamentos de soluções da EDP de primeira ordem, homogênea e com coeficientes constantes, o que é a afirmação do Cartan-Kähler para tableaux.

O algoritmo de Cartan torna-se: 


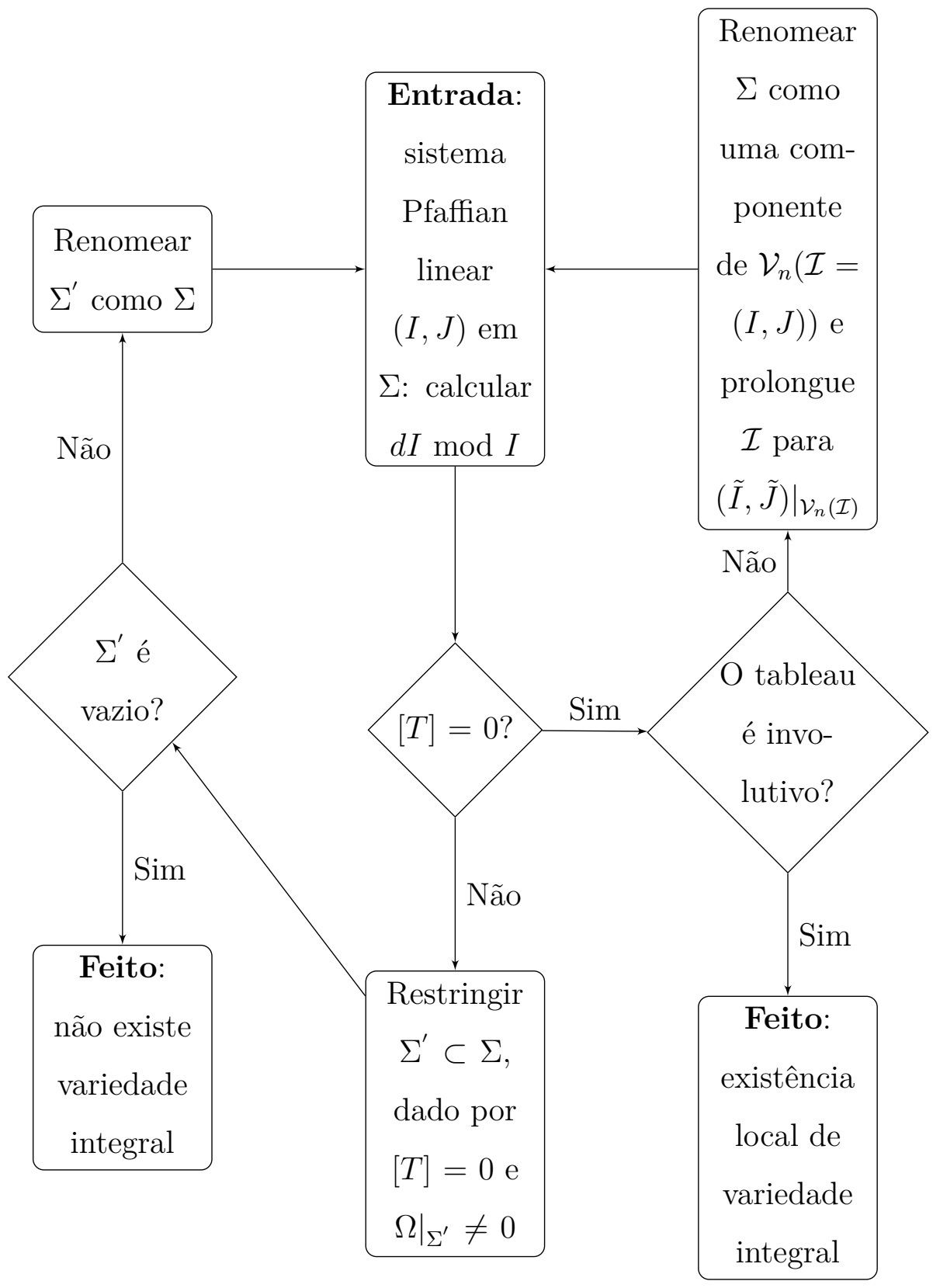

Em palavras:

Sejam $(I, J)$ sistema Pfaffian linear em $\Sigma$ e $x \in \Sigma$ ponto qualquer.

(1) Escolha geradores $\theta^{a}, \omega^{i} \in \Omega^{1}(\Sigma)$ de $I=\left\{\theta^{a}\right\}$ e $J=\left\{\theta^{a}, \omega^{i}\right\}$, complete com $\left\{\pi^{\epsilon}\right\}$ para um correferencial de $T^{*} \Sigma$. Defina $V^{*}=(J / I)_{x}$ e $W=I_{x}$, denote $v^{i}=\omega_{x}^{i}$ e $w_{a}=\theta^{a}$.

(2) Compute $d \theta^{a} \bmod I$, isto é,

$$
d \theta^{a} \equiv A_{\epsilon i}^{a} \pi^{\epsilon} \wedge \omega^{i}+\frac{1}{2} T_{i j}^{a} \omega^{i} \wedge \omega^{j} \bmod I
$$

Defina o tableau em $x$ por

$$
A=A_{x}=\operatorname{span}\left\{A_{\epsilon i}^{a} w_{a} \otimes v^{i}: \epsilon \in\{1, \cdots, \operatorname{dim}(\Sigma)-\operatorname{dim}(J)\}\right\}
$$


Sejam $\delta: W \otimes V^{*} \otimes V^{*} \rightarrow W \otimes \Lambda^{2}\left(V^{*}\right)$ aplicação de anti-simetrização e $H^{(0,2)}(A):=$ $W \otimes \Lambda^{2}\left(V^{*}\right) / \delta\left(A \otimes V^{*}\right)$. A torção em $x$ é

$$
[T]_{x}:=\left[T_{i j}^{a} w_{a} \otimes v^{i} \wedge v^{j}\right] \in H^{(0,2)}\left(A_{x}\right)
$$

(3) Se $[T]_{x} \neq 0$, então renomeie $\Sigma$ pelo conjuntos dos pontos tais que $[T]=0$ e $\omega^{1} \wedge \cdots \wedge \omega^{n} \neq 0$, isto é, $J / I$ com posto $n$ em $\Sigma$.

(4) Suponha $[T]=0$. Seja $A^{(1)}=\left(A \otimes V^{*}\right) \cap\left(W \otimes S^{2}\left(V^{*}\right)\right)$ o prolongamento de $A$. É sempre válida a desigualdade $\operatorname{dim}\left(A^{(1)}\right) \leq s_{1}+2 s_{2} \cdots+n s_{n}$; se vale a igualdade, então $A$ é involutivo, como estamos assumindo $[T]=0$, pelo Cartan-Kähler para sistemas Pfaffian lineares, existe variedade integral de $(I, J)$ passando por $x$.

(5) Se $A$ não é involutivo, precisamos explicitar a Definição 3.2.19 para o caso Pfaffian linear.

Sejam $p \in \Sigma$ e $(I, J)$ sistema diferencial exterior Pfaffian linear em $\Sigma$. Denote $I=$ $\left\{\theta^{1}, \cdots, \theta^{s}\right\}, J=\left\{\omega^{1}, \cdots, \omega^{n}\right\}$ e $\Omega=\omega^{1} \wedge \cdots \wedge \omega$ condição de independência, em que $(I, J)$ satisfaz $d \theta^{a}=\pi_{i}^{a} \wedge \omega^{i} \bmod I$. Recordando a definição de $\mathcal{V}_{n}(\mathcal{I})_{p}=\left\{E \in G_{n}\left(T_{p} \Sigma\right)\right.$ : $\left.\alpha\right|_{E}=0$ e $\left.\left.\Omega\right|_{E} \neq 0, \forall \alpha \in \mathcal{I}\right\}$, equivalentemente, o conjunto $\operatorname{dos} E \in G_{n}\left(T_{p} \Sigma\right)$ tais que $\left.\theta^{a}\right|_{E}=0,\left.d \theta^{a}\right|_{E}=0, \forall a \in\{1, \cdots, s\}$ e $\left.\Omega\right|_{E} \neq 0$.

Seja $E \in \mathcal{V}_{n}(\mathcal{I})_{p}$. Então a condição de independência diz $\left.\left(\omega^{1} \wedge \cdots \wedge \omega^{n}\right)\right|_{E} \neq 0$, isto é, $\left.\omega^{1}\right|_{E}, \cdots,\left.\omega^{n}\right|_{E}$ é linearmente independente em $E^{*}$. Do fato que $E$ é elemento integral temos $\left.\theta^{a}\right|_{E}=0$ e $\left.\left(d \theta^{a}\right)\right|_{E}=0$. Como $(I, J)$ é Pfaffian linear segue $0=\left.d \theta^{a}\right|_{E}=\left.\left.\pi_{i}^{a}\right|_{E} \wedge \omega^{i}\right|_{E}$. Pelo Lema de Cartan existem $p_{i j}^{a}$, simétricos nos índices, tais que $\left.\pi_{i}^{a}\right|_{E}=\left.p_{i j}^{a} \omega^{j}\right|_{E}$. Vamos mostrar que se $B_{a}^{r i}$ é relação de símbolos do tableau associado a $(I, J)$, então $B_{a}^{r i} p_{i j}^{a}=$ 0 , para cada $j=1, \cdots, n$ e $r=1, \cdots, R$. De fato, por construção $\pi_{i}^{a}=A_{\epsilon i}^{a} \pi^{\epsilon}$ para $\left\{\pi^{\epsilon}\right\}$ complemento de $\left\{\theta^{a}, \omega^{i}\right\}$ a um correferencial. Da definição de relação de símbolos, $B_{a}^{r i} A_{\epsilon i}^{a}=0$, para cada $\epsilon$ e $r$. Então

$$
\begin{aligned}
0 & =\left.B_{a}^{r i} A_{\epsilon i}^{a} \pi^{\epsilon}\right|_{E} \\
& =\left.B_{a}^{r i} \pi_{i}^{a}\right|_{E} \\
& =\left.B_{a}^{r i} p_{i j}^{a} \omega^{j}\right|_{E} .
\end{aligned}
$$

Usando novamente o fato de $\left.\omega^{1}\right|_{E}, \cdots,\left.\omega^{n}\right|_{E}$ serem linearmente independentes, segue que $B_{a}^{r i} p_{i j}^{a}=0$, para cada $j$ e $r$. Assim a cada $E \in \mathcal{V}_{n}(\mathcal{I})$, associamos $p_{i j}^{a} w_{a} \otimes v^{i} v^{j} \in A^{(1)}$.

Reciprocamente, seja $p_{i j}^{a} w_{a} \otimes v^{i} v^{j} \in A^{(1)}$, e defina $\widetilde{\pi}_{i}^{a}=\pi_{i}^{a}-p_{i j}^{a} \omega^{j}$. Dado um completamento de $(I, J)$ a um correferencial, novamente denotado $\left\{\pi^{\epsilon}\right\}$, temos que $\pi_{i}^{a}=A_{\epsilon i}^{a} \pi^{\epsilon}$, 
$\operatorname{logo}\left\{\theta^{a}, \omega^{i}, \pi_{i}^{a}\right\}$ também é correferencial, assim como $\left\{\theta^{a}, \omega^{i}, \widetilde{\pi}_{i}^{a}\right\}$. Observe que $d \theta^{a}=$ $\pi_{i}^{a} \wedge \omega^{i}=\left(\pi_{i}^{a}-p_{i j}^{a} \omega^{j}\right) \wedge \omega^{i}=\widetilde{\pi}_{i}^{a} \omega^{i}$ devido a simetria nos índices de $p_{i j}^{a}$, e como $B_{a}^{r i} p_{i j}^{a}=0$, o tableau gerado por $\pi_{i}^{a}$ é o mesmo gerado por $\widetilde{\pi}_{i}^{a}$, defina $E=\left\{\theta^{a}, \omega^{i}, \widetilde{\pi}_{i}^{a}\right\}^{\perp}$, claramente $\operatorname{dim}(E)=n$ e $\left.\theta^{a}\right|_{E}=0$. Para que $E$ pertença a $V_{n}(\mathcal{I})_{p}$ basta $\left.\left(d \theta^{a}\right)\right|_{E}=0$. Verificando esse fato; $\left.\left(d \theta^{a}\right)\right|_{E}=\left.\left.\widetilde{\pi}_{i}^{a}\right|_{E} \wedge \omega^{i}\right|_{E}$; como $E$ é anulado por $\widetilde{\pi}_{i}^{a}$, segue $\left.\left(d \theta^{a}\right)\right|_{E}=0$, isto é, a cada $p_{i j}^{a} w_{a} \otimes v^{i} v^{j}$ associamos um $E \in \mathcal{V}_{n}(\mathcal{I})_{p}$.

Com o exposto acima podemos identificar $\mathcal{V}_{n}(\mathcal{I}) \operatorname{com} \Sigma \times A^{(1)}:=\left\{\left(p, A_{p}^{(1)}\right): p \in \Sigma\right\}$ e o prolongamento do sistema original ao conjunto dos elementos integrais com o sistema $\left(\tilde{I}=\left\{\theta^{a}, \tilde{\pi}_{i}^{a}=A_{\epsilon i}^{a} \pi^{\epsilon}-p_{i j}^{a} \omega_{j}\right\}, \tilde{J}=\left\{\theta^{a}, \omega^{i}, \pi^{\epsilon}\right\}\right)$ em $\Sigma \times A^{(1)}$, permanecendo $\Omega$ como condição de independência. Observe que a construção acima generaliza a Observação 3.2.13.

Exemplo 3.2.22. Problema do mergulho isométrico local.

O objetivo é desse exemplo é exibir uma versão simplificada do seguinte teorema.

Teorema 3.2.23. (Cartan-Janet (1927)). Seja $\left(M^{n}, g\right)$ uma variedade Riemanniana analítica. Então para cada ponto de $M$ existem uma vizinhança $U$ desse ponto e um

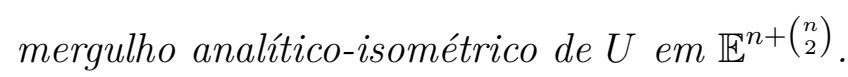

Note que o teorema acima é local e diz a respeito de variedades analíticas. Para o caso global e nas categorias analíticas e $C^{k}$ temos o famoso Teorema do Mergulho de Nash:

Teorema. (Mergulho de Nash (1956)). Seja $\left(M^{n}, g\right)$ uma variedade Riemanniana analítica ou de classe $C^{k}$, em que $3 \leq k \leq \infty$. Então existe um mergulho global, analítico ou de classe $C^{k}$, de $M$ em algum $\mathbb{E}^{N}$, com $N$ satisfazendo: $N \leq \frac{1}{2} n(3 n+11)$, caso $M$ seja compacta, e $N \leq \frac{1}{2} n\left(3 n^{2}+7 n+11\right)+(2 n+1)$ no caso não compacto.

Entretanto a versão $C^{k}$ do teorema de Cartan-Janet, isto é, a existência mergulhos locais para variedades Riemannianas de classe $C^{k}$ em $\mathbb{E}^{n+\left(\begin{array}{c}n \\ 2\end{array}\right)}$, é um problema em aberto. Voltemos ao exemplo.

Sejam $r \in \mathbb{N}, \mathcal{F}_{M}$ fibrado de referenciais ortogonais em $M$ e $\mathcal{F}_{n}\left(\mathbb{E}^{n+r}\right)=\mathbb{E}^{n+r} \times$ $\frac{S O(n+r)}{S O(r)}$ o fibrado $n$-uplas de vetores ortonormais em $\mathbb{E}^{n+r}$. Para fixar notação: $1 \leq$ $i, j \leq n$ e $n+1 \leq \mu, \nu \leq n+r$; recordamos as equações de estrutura:

$\operatorname{para} \mathcal{F}_{M}$ :

$$
\left\{\begin{array}{l}
d \eta^{i}=-\eta_{k}^{i} \wedge \eta^{k} \\
d \eta_{j}^{i}=-\eta_{l}^{i} \wedge \eta_{j}^{l}+\frac{1}{2} R_{j k l}^{i} \eta^{k} \wedge \eta^{l}
\end{array}\right.
$$


em que $R_{j k l}^{i}: \mathcal{F}_{M} \rightarrow \mathbb{R}$ são os coeficientes do tensor de curvatura de $M$.

Análoga à construção da forma de Maurer-Cartan, temos para $\mathcal{F}_{n}\left(\mathbb{E}^{n+r}\right)$ formas $\omega^{i}, \omega^{\mu}$, $\omega_{j}^{i}, \omega_{i}^{\mu}, \omega_{\nu}^{j}, \omega_{\nu}^{\mu} \in \Omega^{1}\left(U \subset \mathcal{F}_{n}\left(\mathbb{E}^{n+r}\right)\right), U$ aberto, satisfazendo $\omega_{j}^{i}=-\omega_{i}^{j}, \omega_{\mu}^{i}=-\omega_{i}^{\mu}$, $\omega_{\nu}^{\mu}=-\omega_{\mu}^{\nu}$, o conjunto $\left\{\omega^{i}, \omega^{\mu}, \omega_{j}^{i}, \omega_{i}^{\mu}\right\}_{i<j}$ é correferencial e verificando as equações de estrutura:

$$
\left\{\begin{array}{l}
d \omega^{i}=-\omega_{j}^{i} \wedge \omega^{j}-\omega_{\mu}^{i} \wedge \omega^{\mu}, \\
d \omega^{\mu}=-\omega_{j}^{\mu} \wedge \omega^{j}-\omega_{\nu}^{\mu} \wedge \omega^{\nu}, \\
d \omega_{j}^{i}=-\omega_{k}^{i} \wedge \omega_{j}^{k}-\omega_{\mu}^{i} \wedge \omega_{j}^{\mu}
\end{array}\right.
$$

Lema 3.2.24. Sejam $\left(I=\left\{\omega^{\mu}, \omega^{i}-\eta^{i}\right\}, J=\left\{\omega^{\mu}, \omega^{i}, \eta^{j}\right\}\right)$ em $\Sigma=\mathcal{F}_{M} \times U$ e $\pi$ : $\mathcal{F}_{M} \rightarrow M, \rho: \mathcal{F}_{n}\left(\mathbb{E}^{n+r}\right) \rightarrow \mathbb{E}^{n+r}$ respectivas projeções. Então as variedades integrais de $(I, J)$, i.e., variedades integrais de $\left\{\omega^{\mu}, \omega^{i}-\eta^{i}\right\}_{\text {diff }}$ com $\Omega=\eta^{1} \wedge \cdots \wedge \eta^{n}$ condição de independência, são localmente gráficos de funções $f \circ \pi$, em que $f: M \rightarrow U$ tem a propriedade: $\rho \circ f: M \rightarrow \mathbb{E}^{n+r}$ é mergulho isométrico local. Reciprocamente, se u : $M \rightarrow \mathbb{E}^{n+r}$ é mergulho isométrico local, então o gráfico de $\widetilde{u \circ \pi}: \mathcal{F}_{M} \rightarrow U, \widetilde{u \circ \pi}(x)=$ $\left(u(\pi(x)), d(u \circ \pi)_{x}\left(E_{1}(x)\right), \cdots, d(u \circ \pi)_{x}\left(E_{n}(x)\right)\right)$, em que $\left\{E^{i}\right\}$ é dual de $\left\{\eta^{i}\right\}$, é variedade integral de $(I, J)$.

Demonstração. [Robert L. Bryant, 1991, p. 95-97].

Calculando $d I \bmod J$

$$
\left\{\begin{array}{l}
d \omega^{\mu}=-\omega_{j}^{\mu} \wedge \omega^{j}-\omega_{\nu}^{\mu} \wedge \omega^{\nu} \equiv 0 \bmod J \\
d\left(\omega^{i}-\eta^{i}\right)=-\omega_{j}^{i} \wedge \omega^{j}-\omega_{\mu}^{i} \wedge \omega^{\mu}+\eta_{k}^{i} \wedge \eta^{k} \equiv 0 \bmod J
\end{array}\right.
$$

O sistema é linear de Pfaff e podemos iniciar o algoritmo para $(I, J)$.

Calculando $d I \bmod I$

$$
\left\{\begin{array}{l}
d \omega^{\mu} \equiv-\omega_{j}^{\mu} \wedge \omega^{j} \bmod I \\
d\left(\omega^{i}-\eta^{i}\right) \equiv-\omega_{j}^{i} \wedge \omega^{j}+\eta_{j}^{i} \wedge \eta^{j} \bmod I
\end{array}\right.
$$

Temos

$$
d\left(\omega^{i}-\eta^{i}\right) \equiv-\omega_{j}^{i} \wedge(\underbrace{\omega^{j}-\eta^{j}}_{0 \bmod I}+\eta^{j})+\eta_{j}^{i} \wedge \eta^{j} \bmod I=\left(-\omega_{j}^{i}+\eta_{j}^{i}\right) \wedge \eta^{j} \bmod I .
$$


Do fato que $\eta^{j}, \omega_{j}^{\mu}$ e $\omega_{j}^{i}-\eta_{j}^{i}$ são linearmente independentes, segue que a torção é nula. Em particular $\left\{\omega_{j}^{\mu}, \omega_{j}^{i}, \eta_{j}^{i}\right\}_{i<j}$ é o complemento $\left\{\pi^{\epsilon}\right\}$. De (3.2) e (3.3) temos

$$
A=\left\{\left(\begin{array}{c}
a_{j}^{\mu} \\
a_{j}^{i}
\end{array}\right): a_{j}^{\mu}, a_{j}^{i} \in \mathbb{R} \text { e } a_{j}^{i}=-a_{i}^{j}\right\} .
$$

Claramente $s_{1}=$ dimensão da primeira coluna de $a_{j}^{\mu}+$ dimensão da primeira coluna de $a_{j}^{i}=r+(n-1)$ e em geral $s_{j}=r+(n-j)$. Por definição $V^{*}=(J / I)_{x}$ e $W=I_{x}$. Escrevendo $w_{i}=\omega^{i}-\eta^{i}$ para $i \leq n$ e $w_{\mu}=\omega^{\mu}$ para $n+1 \leq \mu \leq n+r, v^{i}=\eta^{i}$. Temos que um elemento de $A^{(1)}$ é da forma $\left(a_{j k}^{i} w_{i}+a_{j k}^{\mu} w_{k}\right) \otimes v^{i} v^{k}$, note que

$$
a_{j k}^{i}=-a_{i k}^{j}=-a_{k i}^{j}=a_{j i}^{k}=a_{i j}^{k}=-a_{k j}^{i}=-a_{j k}^{i},
$$

portanto $a_{j k}^{i}=0$, segue $\operatorname{dim}\left(A^{(1)}\right)=r\left(\begin{array}{c}n+1 \\ 2\end{array}\right)=r \frac{n(n+1)}{2}$. Por outro lado $\sum_{j=1}^{n} j s_{j}=$ $j(r+(n-j))=r \frac{n(n+1)}{2}+\sum_{j=1}^{n} j(n-j)$. Concluímos que $A$ não é involutivo.

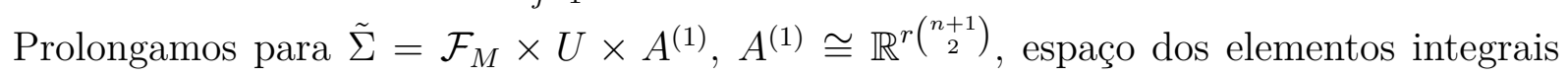
como no passo (5) do algoritmo. Os novos ideais são $\tilde{I}=\left\{\omega^{\mu}, \omega^{i}-\eta^{i}, \omega_{j}^{i}-\eta_{j}^{i}, \omega_{j}^{\mu}-h_{j k}^{\mu} \eta^{k}\right\}$ e $\tilde{J}=\left\{\omega^{\mu}, \omega^{j}, \eta^{j}, \omega_{j}^{i}-\eta_{j}^{i}, \omega_{j}^{\mu}\right\}$.

Calculando $d \tilde{I} \bmod \tilde{I}$ :

$$
\begin{aligned}
d \omega^{\mu} & \equiv-\omega_{j}^{\mu} \wedge \omega^{j} \bmod \tilde{I} \\
& \equiv-(\underbrace{\omega_{j}^{\mu}-h_{j k}^{\mu} \eta^{k}}_{0 \bmod \tilde{I}}+h_{j k}^{\mu} \eta^{k}) \wedge \eta^{j} \bmod \tilde{I} \\
& \equiv-h_{j k}^{\mu} \eta^{k} \wedge \eta^{j} \bmod \tilde{I} \\
& \equiv 0 \bmod \tilde{I}
\end{aligned}
$$

a última equivalência segue da simetria nos índices inferiores e anti-simetria no produto exterior $\eta^{k} \wedge \eta^{j}$. Continuamos,

$$
\begin{aligned}
d\left(\omega^{i}-\eta^{i}\right) & \equiv(\underbrace{-\omega_{j}^{i}+\eta_{j}^{i}}_{0 \bmod \tilde{I}}) \wedge \eta^{j} \bmod \tilde{I} \\
& \equiv 0 \bmod \tilde{I} .
\end{aligned}
$$

$d\left(\omega_{j}^{i}-\eta_{j}^{i}\right)=d \omega_{j}^{i}-d \eta_{j}^{i} ;$ calculando separadamente cada termo,

$$
\begin{aligned}
d \omega_{j}^{i} & \equiv-\omega_{k}^{i} \wedge \omega_{j}^{k}-\omega_{\mu}^{i} \wedge \omega_{j}^{\mu} \bmod \tilde{I} \\
& \equiv-\omega_{k}^{i} \wedge \omega_{j}^{k}-\left(-\omega_{i}^{\mu}+h_{i k}^{\mu} \eta^{k}-h_{i k}^{\mu} \eta^{k}\right) \wedge\left(-\omega_{j}^{\mu}+h_{j l}^{\mu} \eta^{l}-h_{j l}^{\mu} \eta^{l}\right) \bmod \tilde{I} \\
& \equiv-\omega_{k}^{i} \wedge \omega_{j}^{k}+h_{i k}^{\mu} h_{j l}^{\mu} \eta^{k} \wedge \eta^{l} \bmod \tilde{I} .
\end{aligned}
$$




$$
\begin{aligned}
d \eta_{j}^{i} & \equiv-\eta_{l}^{i} \wedge \eta_{j}^{l}+\frac{1}{2} R_{j k l}^{i} \eta^{k} \wedge \eta^{l} \bmod \tilde{I} \\
& \equiv \frac{1}{2} R_{j k l}^{i} \eta^{k} \wedge \eta^{l}-\left(\eta_{l}^{i}+\omega_{l}^{i}-\omega_{l}^{i}\right) \wedge\left(\eta_{j}^{l}+\omega_{j}^{l}-\omega_{j}^{l}\right) \bmod \tilde{I} \\
& \equiv \frac{1}{2} R_{j k l}^{i} \eta^{k} \wedge \eta^{l}-\omega_{l}^{i} \wedge \omega_{k}^{l} \bmod \tilde{I}
\end{aligned}
$$

Fazendo a diferença,

$$
\begin{aligned}
d\left(\omega_{j}^{i}-\eta_{j}^{i}\right) & \equiv-\omega_{k}^{i} \wedge \omega_{j}^{k}+h_{i k}^{\mu} h_{j l}^{\mu} \eta^{k} \wedge \eta^{l}-\left(\frac{1}{2} R_{j k l}^{i} \eta^{k} \wedge \eta^{l}-\omega_{l}^{i} \wedge \omega_{k}^{l}\right) \bmod \tilde{I} \\
& \equiv \sum_{k<l}\left(\sum_{\mu}\left(h_{i k}^{\mu} h_{j l}^{\mu}-h_{i l}^{\mu} h_{j k}^{\mu}\right)-R_{j k l}^{i}\right) \eta^{k} \wedge \eta^{l} \bmod \tilde{I}
\end{aligned}
$$

isto é, $(\tilde{I}, \tilde{J})$ tem torção. Como em (3), passamos a $\Sigma^{\prime}$ tal que

$$
\sum_{\mu}\left(h_{i k}^{\mu} h_{j l}^{\mu}-h_{i l}^{\mu} h_{j k}^{\mu}\right)=R_{j k l}^{i}
$$

e continue valendo a condição de independência. Note que (3.4) é a equação de Gauss. Falta calcular $d\left(\omega_{j}^{\mu}-h_{j k}^{\mu} \eta^{k}\right) \bmod \tilde{I}$,

$$
\begin{aligned}
d\left(\omega_{j}^{\mu}-h_{j k}^{\mu} \eta^{k}\right) & \equiv d \omega_{j}^{\mu}-d h_{j k}^{\mu} \wedge \eta^{k}-h_{j k}^{\mu} d \eta^{k} \bmod \tilde{I} \\
& \equiv d \omega_{j}^{\mu}-d h_{j k}^{\mu} \wedge\left(\eta^{k}-\omega^{k}+\omega^{k}\right)-h_{j k}^{\mu} d\left(\eta^{k}-\omega^{k}+\omega^{k}\right) \bmod \tilde{I} \\
& \equiv d \omega_{j}^{\mu}-d h_{j k}^{\mu} \wedge \underbrace{\left(\eta^{k}-\omega^{k}\right)}_{0 \bmod \tilde{I}}-d h_{j k}^{\mu} \wedge \omega^{k}-h_{j k}^{\mu} \underbrace{d\left(\eta^{k}-\omega^{k}\right)}_{0 \bmod \tilde{I}}-h_{j k}^{\mu} \omega^{k} \bmod \tilde{I} \\
& \equiv d \omega_{j}^{\mu}-d h_{j k}^{\mu} \wedge \omega^{k}-h_{j k}^{\mu} \omega^{k} \bmod \tilde{I} \\
& \equiv-\omega_{l}^{\mu} \wedge \omega_{j}^{l}-\omega_{\nu}^{\mu} \wedge \omega_{j}^{\nu}-d h_{j k}^{\mu} \wedge \omega^{k}-h_{j k}^{\mu}(-\omega_{s}^{k} \wedge \omega^{s}-\underbrace{\omega_{\nu}^{k} \wedge \omega^{\nu}}_{0 \bmod \tilde{I}}) \bmod \tilde{I} \\
& \equiv-h_{l k}^{\mu} \omega^{k} \wedge \omega_{j}^{l}-\omega_{\nu}^{\mu} \wedge\left(h_{j k}^{\nu} \omega^{k}\right)-d h_{j k}^{\mu} \wedge \omega^{k}+h_{j k}^{\mu} \omega_{s}^{k} \wedge \omega^{s} \bmod \tilde{I} \\
& \equiv\left(h_{l k}^{\mu} \omega_{j}^{l}-h_{j k}^{\nu} \omega_{\nu}^{\mu}-d h_{j k}^{\nu}+h_{j s}^{\nu} \omega_{k}^{s}\right) \wedge \omega^{k} \bmod \tilde{I} .
\end{aligned}
$$

Definimos $\pi_{j k}^{\mu}:=h_{l k}^{\mu} \omega_{j}^{l}-h_{j k}^{\nu} \omega_{\nu}^{\mu}-d h_{j k}^{\nu}+h_{j s}^{\nu} \omega_{k}^{s}$. Então $d\left(\omega_{j}^{\mu}-h_{j k}^{\mu} \eta^{k}\right)=\pi_{j k}^{\mu} \wedge \omega^{k} \bmod \tilde{I}$.

Especifiquemos para caso $n=2$ e $r=1$. Note que para esse caso (3.4) é

$$
R=R_{212}^{1}=h_{11} h_{22}-\left(h_{12}\right)^{2}
$$

onde omitimos $\mu$ pois $r=1$. Denote $\theta_{j}=\omega_{j}^{3}-h_{j k} \omega^{k} ;$ segue que

$$
\begin{aligned}
d \theta_{1} & \equiv\left(-d h_{11}+(\underbrace{h_{11} \omega_{1}^{1}}_{0}+h_{12} \omega_{1}^{2})+(\underbrace{h_{11} \omega_{1}^{1}}_{0}+h_{12} \omega_{1}^{2})\right) \wedge \omega^{1} \\
& +\left(-d h_{12}+(h_{11} \omega_{2}^{1}+\underbrace{h_{12} \omega_{2}^{2}}_{0})+(\underbrace{h_{21} \omega_{1}^{1}}_{0}+h_{22} \omega_{1}^{2})\right) \wedge \omega^{2} \bmod \tilde{I} \\
& \equiv\left(-d h_{11}+2 h_{12} \omega_{1}^{2}\right) \wedge \omega^{1}+\left(-d h_{12}-\left(h_{11}-h_{22}\right) \omega_{1}^{2}\right) \wedge \omega^{2} \bmod \tilde{I}
\end{aligned}
$$


Analogamente para $d \theta_{2}$, e podemos escrever

$$
d\left(\begin{array}{c}
\theta^{1} \\
\theta^{2}
\end{array}\right) \equiv\left(\begin{array}{cc}
-d h_{11}+2 h_{12} \omega_{1}^{2} & -d h_{12}-\left(h_{11}-h_{22}\right) \omega_{1}^{2} \\
-d h_{12}-\left(h_{11}-h_{22}\right) \omega_{1}^{2} & -d h_{22}+2 h_{12} \omega_{1}^{2}
\end{array}\right) \wedge\left(\begin{array}{c}
\omega^{1} \\
\omega^{2}
\end{array}\right) \bmod \tilde{I} .
$$

Vimos na Proposição 1.4.7 que $R$ é constante nas fibras, i.e., o tensor $R$ é básico. Segue que $d R=R_{1} \eta^{1}+R_{2} \eta^{2}$. Como $\omega^{i}-\eta^{i} \in \tilde{I}, d R \equiv R_{1} \omega^{1}+R_{2} \omega^{2} \bmod \tilde{I}$. Derivando (3.5),

$$
R_{1} \omega^{1}+R_{2} \omega^{2}-h_{11} d h_{12}-h_{22} d h_{11}+2 h_{12} d h_{12} \equiv 0 \bmod \tilde{I}
$$

Defina

$$
\left\{\begin{array}{l}
\pi_{1}=-d h_{11}+2 h_{12} \omega_{1}^{2} \\
\pi_{2}=-d h_{12}-\left(h_{11}-h_{22}\right) \omega_{1}^{2} \\
\pi_{3}=-d h_{22}+2 h_{12} \omega_{1}^{2} .
\end{array}\right.
$$

De (3.7) segue

$$
R_{1} \omega^{1}+R_{2} \omega^{2}+h_{11} \pi_{3}+h_{22} \pi_{1}-2 h_{12} \pi_{2} \equiv 0 \bmod \tilde{I}
$$

E isolando $\pi_{3},(3.6)$ torna-se

$$
\begin{aligned}
d\left(\begin{array}{l}
\theta^{1} \\
\theta^{2}
\end{array}\right) & \equiv\left(\begin{array}{cc}
\pi_{1} & \pi_{2} \\
\pi_{2} & \frac{1}{h_{11}}\left(2 h_{12} \pi_{2}-h_{22} \pi_{1}-R_{1} \omega^{1}-R_{2} \omega_{2}\right)
\end{array}\right) \wedge\left(\begin{array}{c}
\omega^{1} \\
\omega^{2}
\end{array}\right) \bmod \tilde{I} \\
& \equiv\left(\left(\begin{array}{cc}
\pi_{1} & \pi_{2} \\
\pi_{2} & \frac{1}{h_{11}}\left(2 h_{12} \pi_{2}-h_{22} \pi_{1}\right)
\end{array}\right)+\left(\begin{array}{cc}
0 & 0 \\
0 & -\frac{1}{h_{11}}\left(R_{1} \omega^{1}+R_{2} \omega^{2}\right)
\end{array}\right)\right) \wedge\left(\begin{array}{l}
\omega^{1} \\
\omega^{2}
\end{array}\right) \bmod \tilde{I} \\
& \equiv\left(\begin{array}{ll}
\pi_{1} & \pi_{2} \\
\pi_{2} & \frac{1}{h_{11}}\left(2 h_{12} \pi_{2}-h_{22} \pi_{1}\right)
\end{array}\right) \wedge\left(\begin{array}{c}
\omega^{1} \\
\omega^{2}
\end{array}\right)+\left(\begin{array}{c}
0 \\
-\frac{R_{1}}{h_{11}} \omega^{1} \wedge \omega^{2}
\end{array}\right) \bmod \tilde{I}
\end{aligned}
$$


Troque o complemento $\tilde{\pi}_{1}=\pi_{1}+\frac{R_{1}}{h_{22}} \omega^{1}$ :

$$
\begin{aligned}
& d\left(\begin{array}{c}
\theta^{1} \\
\theta^{2}
\end{array}\right) \equiv\left(\begin{array}{cc}
\tilde{\pi}_{1}-\frac{R_{1}}{h_{22}} \omega^{1} & \pi_{2} \\
\pi_{2} & \frac{1}{h_{11}}\left(2 h_{12} \pi_{2}-h_{22}\left(\tilde{\pi}_{1}-\frac{R_{1}}{h_{22}} \omega^{1}\right)\right)
\end{array}\right) \wedge\left(\begin{array}{c}
\omega^{1} \\
\omega^{2}
\end{array}\right) \\
& +\left(\begin{array}{c}
0 \\
-\frac{R_{1}}{h_{11}} \omega^{1} \wedge \omega^{2}
\end{array}\right) \bmod \tilde{I} \\
& \equiv\left(\left(\begin{array}{cc}
\tilde{\pi}_{1} & \pi_{2} \\
\pi_{2} & \frac{1}{h_{11}}\left(2 h_{12} \pi_{2}-h_{22} \tilde{\pi}_{1}\right)
\end{array}\right)+\left(\begin{array}{cc}
-\frac{R_{1}}{h_{22}} \omega^{1} & 0 \\
0 & \frac{R_{1}}{h_{11}} \omega^{1}
\end{array}\right)\right) \wedge\left(\begin{array}{c}
\omega^{1} \\
\omega^{2}
\end{array}\right) \\
& +\left(\begin{array}{c}
0 \\
-\frac{R_{1}}{h_{11}} \omega^{1} \wedge \omega^{2}
\end{array}\right) \bmod \tilde{I} \\
& \equiv\left(\begin{array}{cc}
\tilde{\pi}_{1} & \pi_{2} \\
\pi_{2} & \frac{1}{h_{11}}\left(2 h_{12} \pi_{2}-h_{22} \tilde{\pi}_{1}\right)
\end{array}\right) \wedge\left(\begin{array}{c}
\omega^{1} \\
\omega^{2}
\end{array}\right)+\left(\begin{array}{c}
0 \\
\frac{R_{1}}{h_{11}} \omega^{1} \wedge \omega^{2}
\end{array}\right) \\
& +\left(\begin{array}{c}
0 \\
-\frac{R_{1}}{h_{11}} \omega^{1} \wedge \omega^{2}
\end{array}\right) \bmod \tilde{I} \\
& \equiv\left(\begin{array}{cc}
\tilde{\pi}_{1} & \pi_{2} \\
\pi_{2} & \frac{1}{h_{11}}\left(2 h_{12} \pi_{2}-h_{22} \tilde{\pi}_{1}\right)
\end{array}\right) \wedge\left(\begin{array}{c}
\omega^{1} \\
\omega^{2}
\end{array}\right) \bmod \tilde{I}
\end{aligned}
$$

portanto a torção é absorvível. Reescrevendo a equação acima:

$$
\left\{\begin{array}{l}
d \theta^{1} \equiv \tilde{\pi}_{1} \wedge \omega^{1}+0 \tilde{\pi}_{1} \wedge \omega^{2}+0 \pi_{2} \wedge \omega^{1}+\pi_{2} \wedge \omega_{2} \bmod \tilde{I} \\
d \theta^{2} \equiv 0 \tilde{\pi}_{1} \wedge \omega^{1}-\frac{h_{22}}{h_{11}} \tilde{\pi}_{1} \wedge \omega^{2}+\pi_{2} \wedge \omega^{1}+2 \frac{h_{12}}{h_{11}} \pi_{2} \wedge \omega^{2} \bmod \tilde{I}
\end{array}\right.
$$

Concluímos que os coeficientes do novo tableau são $A_{11}^{1}=1, A_{12}^{1}=0, A_{21}^{1}=0, A_{22}^{1}=1$, $A_{11}^{2}=0, A_{12}^{2}=\frac{h_{22}}{h_{11}}=a, A_{21}^{2}=1$ e $A_{22}^{2}=-2 \frac{h_{12}}{h_{11}}=b$, isto é,

$$
A=\operatorname{span}\left\{\left(\begin{array}{ll}
1 & 0 \\
0 & 1
\end{array}\right),\left(\begin{array}{ll}
0 & a \\
1 & b
\end{array}\right)\right\}=\left\{\left(\begin{array}{cc}
\alpha & a \beta \\
\beta & \alpha+b \beta
\end{array}\right): \alpha, \beta \in \mathbb{R}\right\} .
$$

Os caracteres são $s_{1}(A)=2$ e $s_{2}(A)=0$. Resta calcular $\operatorname{dim}\left(A^{(1)}\right)$.

Sejam $w_{a}, v^{i}$ como no passo (1) do algoritmo. Um elemento $p \in A$ é da forma $p=$ 
$p_{i} w_{1} \otimes v^{i}+q_{j} w_{2} \otimes v^{j}$. Pela forma de $A$,

$$
\left\{\begin{array}{l}
p_{1}=\alpha \\
p_{2}=\beta \\
q_{1}=a \beta \\
q_{2}=\alpha+b \beta .
\end{array}\right.
$$

Reduzindo,

$$
\left\{\begin{array}{l}
q_{1}=a p_{2} \\
q_{2}=p_{1}+b p_{2}
\end{array}\right.
$$

De (3.9) segue $p_{i j} w_{1} \otimes v^{i} v^{j}+q_{i j} w_{2} \otimes v^{i} v^{j} \in A^{(1)}$ satisfaz:

$$
\left\{\begin{array}{l}
q_{11}=a p_{21} \\
q_{12}=a p_{22} \\
q_{21}=p_{11}+b p_{21} \\
q_{22}=p_{12}+b p_{22} .
\end{array}\right.
$$

De (3.10) temos que dimensão de $A^{(1)}$ é no máximo 3 , mas do fato que $p_{12}=p_{21}$ e $q_{12}=q_{21}$ vemos que $a p_{22}=p_{11}+b p_{21}$ se e somente se $p_{11}=a p_{22}-b p_{12}$, isto é, $\operatorname{dim}\left(A^{(1)}\right)=2, \operatorname{logo}$ $2=\operatorname{dim}\left(A^{1}\right)=s_{1}(A)+2 s_{2}(A)$, e $A$ é involutivo. Pelo Teorema 3.2.20 existe variedade integral de $(I, J)$ e o Lema 3.2.25 assegura a existência do mergulho isométrico local de $f: M \rightarrow \mathbb{E}^{3}$. Observe que adicionamos as hipóteses de $h_{11} \neq 0$ e $h_{22} \neq 0$, vamos analisar o caso $h_{11}=0$ e $h_{22} \neq 0$.

O primeiro passo é notar que podemos supor $h_{12} \neq 0$, caso contrário $R \equiv 0$ e existe um mergulho isométrico local em $\mathbb{R}^{2}$. De (3.8) obtemos

$$
\pi_{2} \equiv \frac{1}{2 h_{12}}\left(h_{22} \pi_{1}+R_{1} \omega^{1}+R_{2} \omega^{2}\right)
$$

Análogo ao caso $h_{11}, h_{22} \neq 0$, temos

$$
d\left(\begin{array}{l}
\theta_{1} \\
\theta_{2}
\end{array}\right) \equiv\left(\begin{array}{cc}
\pi_{1} & a \pi_{1} \\
a \pi_{1} & \pi_{3}
\end{array}\right) \wedge\left(\begin{array}{c}
\omega^{1} \\
\omega^{2}
\end{array}\right)+\left(\begin{array}{c}
\frac{R_{1}}{2 h_{12}} \omega^{1} \wedge \omega^{2} \\
-\frac{R_{2}}{2 h_{12}} \omega^{1} \wedge \omega^{2}
\end{array}\right) \bmod \tilde{I}
$$

em que $a=\frac{h_{22}}{2 h_{12}}$. Defina $\tilde{\pi}_{1}=\pi_{1}+x \omega^{1}+y \omega^{2}$ e $\tilde{\pi_{3}}=\pi_{3}+z \omega^{1}+t \omega^{2}$, com $x, y, z, t$ a serem determinados: 


$$
\begin{aligned}
d\left(\begin{array}{l}
\theta_{1} \\
\theta_{2}
\end{array}\right) & \equiv\left(\left(\begin{array}{cc}
\tilde{\pi}_{1} & a \tilde{\pi}_{1} \\
a \tilde{\pi}_{1} & \tilde{\pi}_{3}
\end{array}\right)+\left(\begin{array}{cc}
-x \omega^{1}-y \omega^{2} & -a x \omega^{1}-a y \omega^{2} \\
-a x \omega^{1}-a y \omega^{2} & -z \omega^{1}-t \omega^{2}
\end{array}\right)\right) \wedge\left(\begin{array}{l}
\omega^{1} \\
\omega^{2}
\end{array}\right)+ \\
& +\left(\begin{array}{c}
\frac{R_{1}}{2 h_{12}} \omega^{1} \wedge \omega^{2} \\
-\frac{R_{2}}{2 h_{12}} \omega^{1} \wedge \omega^{2}
\end{array}\right) \\
& \equiv\left(\begin{array}{cc}
\tilde{\pi}_{1} & a \tilde{\pi}_{1} \\
a \tilde{\pi}_{1} & \tilde{\pi}_{3}
\end{array}\right) \wedge\left(\begin{array}{l}
\omega^{1} \\
\omega^{2}
\end{array}\right)+\left(\begin{array}{l}
(y-a x) \omega^{1} \wedge \omega^{2} \\
(a y-z) \omega^{1} \wedge \omega^{2}
\end{array}\right)+\left(\begin{array}{c}
\frac{R_{1}}{2 h_{12}} \omega^{1} \wedge \omega^{2} \\
-\frac{R_{2}}{2 h_{12}} \omega^{1} \wedge \omega^{2}
\end{array}\right) .
\end{aligned}
$$

Para não haver torção basta que

$$
\left\{\begin{array}{l}
y-\frac{h_{22}}{2 h_{12}} x=-\frac{R_{1}}{2 h_{12}}, \\
\frac{h_{22}}{2 h_{12}} y-z=\frac{R_{2}}{2 h_{12}} .
\end{array}\right.
$$

Tomando $x=\frac{R_{1}}{h_{22}}$, necessariamente $y=0$ e $z=\frac{R_{2}}{2 h_{12}}$. Os coeficientes do tableau são $A_{11}^{1}=1, A_{12}^{1}=a, A_{31}^{1}=0, A_{32}^{1}=0, A_{11}^{2}=0, A_{12}^{2}=0, A_{31}^{2}=0, A_{32}^{2}=1 \mathrm{e}$

$$
A=\left\{\left(\begin{array}{cc}
\alpha+a \beta & a \alpha \\
0 & \beta
\end{array}\right): \alpha, \beta \in \mathbb{R}\right\}
$$

Ao passar para uma base genérica verificamos $s_{1}(A)=2, s_{2}(A)=0$. Para calcular $A^{(1)}$ :

$$
\left\{\begin{array}{l}
p_{1}=\frac{1}{a} q_{1}+a q_{2} \\
p_{2}=0
\end{array}\right.
$$

Idêntico ao caso anterior, segue

$$
\left\{\begin{array}{l}
p_{11}=\frac{1}{a} q_{11}+a q_{21} \\
p_{12}=\frac{1}{a} q_{12}+a q_{22} \\
p_{21}=0 \\
p_{22}=0
\end{array}\right.
$$

temos $p^{\prime}$ 's em função das $q$ 's, mas como $p_{12}=p_{21}=0$, vale a relação $q_{22}=-\frac{1}{a^{2}} q_{12}$, portanto $\operatorname{dim}\left(A^{(1)}\right)=2$ e $A$ também é involutivo. O mesmo raciocínio aplica-se ao caso $h_{11}, h_{12} \neq 0, h_{22}=0$. Resta apenas o caso $h_{11}=h_{22}=0$ e $h_{12} \neq 0$. Seguindo os passos anteriores, obtemos o tableau

$$
A=\left\{\left(\begin{array}{ll}
\alpha & 0 \\
0 & \beta
\end{array}\right): \alpha, \beta \in \mathbb{R}\right\},
$$


$\operatorname{com} s_{1}(A)=s_{2}(A)=1 \mathrm{e} \operatorname{dim}\left(A^{(1)}\right)=2$, isto é, $2=\operatorname{dim}\left(A^{(1)}\right)<1 \times 1+2 \times 1=3$, não involutivo. Segundo o algorítimo precisamos prolongar, como em (5), e adicionar ao novo ideal apenas as formas $\theta_{1}^{1}=\tilde{\pi}_{1}-p_{11} \omega^{1}$ e $\theta_{2}^{2}=\tilde{\pi}_{3}-q_{22} \omega^{2}$, uma vez que $\theta_{2}^{1}=\theta_{1}^{2}=0$, e reiniciar o algoritmo. O problema que fica é se um sistema Pfaffian linear $(I, J)$ pode ser estendido indefinidamente? A resposta para essa pergunta é não. Kuranishi demonstrou no artigo On E. Cartan's Prolongation Theorem of Exterior Differential Systems [Kuranishi, 1957], em um contexto mais amplo, que após um número finito de prolongamentos o tableau torna-se involutivo ou o espaço dos elementos integrais é vazio. Uma discussão para o caso Pfaffian linear pode ser encontrada no livro [Robert L. Bryant, 1991, p. 255-259], e nas notas [Kuranishi, 1967] encontra-se uma aplicação do resultado de Kuranishi à teoria de EDP.

Exemplo 3.2.25. (Existência de coordenadas isotérmicas). Sejam $(M, g)$ e $(N, h)$ duas variedades Riemannianas com métricas analíticas e ambas de dimensão 2. Considere os fibrados de referenciais ortogonais $\mathcal{F}_{M}, \mathcal{F}_{N}$, e denote as equações de estrutura por $d \omega^{i}=-\omega_{j}^{i} \wedge \omega^{j}$ e $d \eta^{i}=-\omega_{j}^{i} \wedge \omega^{j}$, em que $\left\{\omega^{1}, \omega^{2}, \omega_{2}^{1}\right\},\left\{\eta^{1}, \eta^{2}, \eta_{2}^{1}\right\}$ são correferenciais de $\mathcal{F}_{M}$ e $\mathcal{F}_{N}$ respectivamente.

Defina $\Sigma=\mathcal{F}_{M} \times \mathcal{F}_{N} \times(0, \infty), \pi_{i}$ a projeção em cada fator, $i \in\{1,2,3\},\left(I=\left\{\theta^{1}:=\lambda \omega^{1}-\right.\right.$ $\left.\left.\eta^{1}, \theta^{2}:=\lambda \omega^{2}-\eta^{2}\right\}, J=\left\{\theta^{1}, \theta^{2}, \omega^{1}, \omega^{2}, \omega_{1}^{2}\right\}\right)$, e fixe $y \in \Sigma$. Seja $S$ uma variedade integral de $(I, J)$. Temos $\Omega=\omega^{1} \wedge \omega^{2} \wedge \omega_{1}^{2} \neq 0$ em $S$, logo podemos escrever $S$ localmente como o gráfico de uma função $f: \mathcal{F}_{M} \rightarrow \mathcal{F}_{N} \times(0, \infty)$. Denote $\phi: \mathcal{F}_{M} \rightarrow \mathcal{F}_{N}$ e $\lambda: \mathcal{F}_{M} \rightarrow(0, \infty)$ as funções componentes de $f$, e tome a função gráfico $F: \mathcal{F}_{M} \rightarrow S, F(x)=(x, \phi(x), \lambda(x))$. Seja $i: S \rightarrow \Sigma$ a inclusão. Do fato de que $S$ é variedade integral de $(I, J)$, segue $i^{*}\left(\theta^{j}\right)=0$, $j \in\{1,2\}$, portanto $(i \circ F)^{*}\left(\theta^{j}\right)=F^{*}\left(i^{*}\left(\theta^{j}\right)\right)=0$. Convém omitir o pull-back de $i$, porém explicitar o pull-back por $\pi_{1}^{*}\left(\omega^{j}\right), \pi_{2}^{*}\left(\eta^{j}\right)$ e $\pi_{3}^{*}(\lambda)$ para ficar clara a relação entre as formas. Sejam $x \in \mathcal{F}_{M}$ e $v \in T_{x}\left(\mathcal{F}_{M}\right)$;

$$
\begin{aligned}
0 & =\left(\pi_{3}^{*}(\lambda) F^{*}\left(\pi_{1}^{*}\left(\omega^{j}\right)-\pi_{2}^{*}\left(\eta^{j}\right)\right)\right)_{x}(v) \\
& =\left(F^{*}\left(\pi_{3}^{*}(\lambda) \pi_{1}^{*}\left(\omega^{j}\right)\right)\right)_{x}(v)-\left(F^{*}\left(\pi_{2}^{*}\left(\eta^{j}\right)\right)\right)_{x}(v) \\
& =\left(\left(\pi_{3} \circ F\right)^{*}(\lambda)\right)_{x}(v) \omega_{\pi_{1}(F(x))}^{j}\left(d\left(\pi_{1} \circ F\right)_{x}(v)\right)-\eta_{\pi_{2}(F(x))}^{j}\left(d\left(\pi_{2} \circ F\right)_{x}(v)\right) \\
& =\lambda(x) \omega_{x}^{j}(v)-\eta_{\phi(x)}^{j}\left(d \phi_{x}(v)\right) \\
& =\left(\lambda \omega^{j}-\phi^{*} \eta^{j}\right)_{x}(v)
\end{aligned}
$$

portanto $\phi^{*}\left(\eta^{j}\right)=\lambda \omega^{j}$. Resumidamente, uma variedade integral de $(I, J)$ induz uma 
aplicação local $\phi: \mathcal{F}_{M} \rightarrow \mathcal{F}_{N}$ tal que $\phi^{*}\left(\eta^{j}\right)=\lambda \omega^{j}, \lambda$ função estritamente positiva. Verificamos que $S$ de fato existe,

$$
\begin{aligned}
d \theta^{1} & =d \lambda \wedge \omega^{1}+\lambda d \omega^{1}-d \eta^{1} \\
& =d \lambda \wedge \omega^{1}+\lambda\left(-\omega_{2}^{1} \wedge \omega^{2}\right)-\eta_{2}^{1} \wedge \eta^{2} \\
& \equiv d \lambda \wedge \omega^{1}+\left(\lambda \omega_{1}^{2}+\lambda \eta_{1}^{2}\right) \wedge \omega^{2} \bmod I .
\end{aligned}
$$

Analogamente, $d \theta^{2} \equiv-\lambda\left(\omega_{1}^{2}+\eta_{1}^{2}\right) \wedge \omega^{1}+d \lambda \wedge \omega^{2} \bmod I$. Denote $\pi_{1}=d \lambda$ e $\pi_{2}=\lambda\left(\omega_{1}^{2}+\eta_{1}^{2}\right)$. O conjunto $\left\{\theta^{1}, \theta^{2}, \omega^{1}, \omega^{2}, \omega_{1}^{2}, \pi^{1}, \pi^{2}\right\}$ é correferencial de $T^{*}(\Sigma)$, e

$$
\left\{\begin{array}{l}
d \theta^{1} \equiv \pi^{1} \wedge \omega^{1}+\pi^{2} \wedge \omega^{2} \bmod I \\
d \theta^{2} \equiv-\pi^{2} \wedge \omega^{1}+\pi^{1} \wedge \omega^{2} \bmod I .
\end{array}\right.
$$

A torção é nula e os coeficiêntes do tableau são $A_{11}^{1}=1, A_{12}^{1}=0, A_{21}^{1}=0, A_{22}^{1}=1$, $A_{11}^{2}=0, A_{12}^{2}=1, A_{21}^{2}=-1$ e $A_{22}^{2}=0$. Concluímos que o tableau $A$ é dado por

$$
A=\left\{\left(\begin{array}{cc}
\alpha & \beta \\
-\beta & \alpha
\end{array}\right): \alpha, \beta \in \mathbb{R}\right\}
$$

Temos $s_{1}(A)=2, s_{2}(A)=0$. Passamos ao cômputo de $\operatorname{dim}\left(A^{(1)}\right)$. Seja $\left(\begin{array}{ll}p_{1} & q_{1} \\ p_{2} & q_{2}\end{array}\right) \in A$. Então

$$
\left\{\begin{array}{l}
p_{1}=q_{2} \\
q_{1}=-p_{2}
\end{array}\right.
$$

Note que as equações acima são simplesmente a equações de Cauchy-Riemann, e essas relações impõem a $A^{(1)}$

$$
\left\{\begin{array}{l}
p_{11}=q_{21} \\
p_{12}=q_{22} \\
q_{11}=-p_{21} \\
q_{12}=-p_{22}
\end{array}\right.
$$

Novamente temos $q^{\prime} s$ em função dos $p^{\prime} s, p_{11}=q_{21}=q_{12}=-p_{22}$, portanto $\operatorname{dim}\left(A^{(1)}\right)=2$ e $A$ involutivo. Pelo Teorema 3.2.20 existe variedade integral de $(I, J)$ passando por $y$. Seja $s: M \rightarrow \mathcal{F}_{M}$ seção local tal que sua imagem contenha $\pi_{1}(y)$, considere $\phi$ induzida por $S$, variedade integral, e defina $\Phi: M \rightarrow N, \Phi=\rho \circ \phi \circ s$, em que $\rho$ é projeção de 
$\mathcal{F}_{N}$. Vimos no Capítulo 1 que $h=t^{*}\left(\left(\eta^{1}\right)^{2}+\left(\eta^{2}\right)^{2}\right)$, para $t$ qualquer seção local, então $\rho^{*}(h)=\left(\eta^{1}\right)^{2}+\left(\eta^{2}\right)^{2}, \operatorname{assim}$

$$
\begin{aligned}
\Phi^{*}(h) & =s^{*}\left(\phi^{*}\left(\left(\eta^{1}\right)^{2}+\left(\eta^{2}\right)^{2}\right)\right) \\
& =s^{*}\left(\lambda^{2}\left(\left(\omega^{1}\right)^{2}+\left(\omega^{2}\right)^{2}\right)\right) \\
& =\lambda^{2} g .
\end{aligned}
$$

Vamos agora à existência de coordenadas isotérmicas. No contexto acima, tome $N=\mathbb{R}^{2}$ e $h$ métrica Euclidiana, então para cada $p \in M$, existe coordenada local $(x, y)$, dada pela $\Phi$, tal que $g=\beta^{2}\left(d x^{2}+d y^{2}\right)$, em que $\beta=\frac{1}{\lambda}$ na construção anterior.

\subsection{Cartan-Kähler geral}

Dedicaremos esta seção à apresentação da versão geral do Teorema de Cartan-Kähler e a aplicação desse à superfícies de Weingarten. No que segue vamos assumir que os sistemas diferenciais exteriores $\mathcal{I}$ em $\Sigma$ não possuem funções (0-formas), caso contrário passaremos ao conjunto dos pontos em $\Sigma$ tais que todas essas funções são identicamente nulas. Nosso objetivo agora é obter condições para que um dado $E \in \mathcal{V}_{n}(\mathcal{I})$ seja tangente a uma variedade integral de $\mathcal{I}$.

Sejam $p \in \Sigma$ e $E \in G_{n}\left(T_{p} \Sigma\right)$, e tome um sistema de coordenadas $\left(x^{1}, \cdots, x^{n}\right.$, $\left.y^{1}, \cdots, y^{s}\right)$ de $\Sigma$ em torno de $p$ tal que

$$
E=\operatorname{span}\left\{\frac{\partial}{\partial x^{1}}, \cdots, \frac{\partial}{\partial x^{n}}\right\},
$$

em particular o conjunto $\left\{\frac{\partial}{\partial x^{1}}, \cdots, \frac{\partial}{\partial x^{n}}\right\}$ é linearmente independente, logo $\left(d x^{1} \wedge \cdots \wedge\right.$ $\left.d x^{n}\right)\left.\right|_{E} \neq 0$. Por continuidade existe uma vizinhança $U$ de $E$ em $G_{n}\left(T_{p} \Sigma\right)$ tal que $\left(d x^{1} \wedge\right.$ $\left.\cdots \wedge d x^{n}\right)\left.\right|_{\widetilde{E}} \neq 0$, para cada $\widetilde{E} \in U$. Como $\operatorname{dim}(\widetilde{E})=n$, existem funções $p_{i}^{a}: U \rightarrow \mathbb{R}$ tais que

$$
\left.d y^{a}\right|_{\widetilde{E}}=\left.p_{i}^{a} d x^{i}\right|_{\widetilde{E}}
$$

Agora $\left(U,\left(x^{i}, y^{a}, p_{i}^{a}\right)\right)$ forma um sistema de coordenadas em torno de $E$. Vimos na Definição 1.1 .3 que $E \in \mathcal{V}_{n}(\mathcal{I})$ se, e somente se, $\left.\alpha\right|_{E}=0$ para cada $\alpha \in \mathcal{I}^{n}$. Denote $\alpha=\alpha_{I J} d x^{I} \wedge d y^{J}$, em que $\alpha_{I J}$ são funções diferenciáveis e $I, J$ são multi-índices crescentes com $|I|+|J|=n$. Por (3.11),

$$
\left.\alpha\right|_{\widetilde{E}}=\left.\left.\sum_{L} \alpha_{I J} p_{l_{1}}^{j_{1}} \cdots p_{l_{k}}^{j_{k}} d x^{I}\right|_{\widetilde{E}} \wedge d x^{L}\right|_{\widetilde{E}}=\left.F^{\alpha} d x^{1} \wedge \cdots \wedge d x^{n}\right|_{\widetilde{E}},
$$


em que $I, L$ são multi-índices crescentes. Segue $\widetilde{E} \in \mathcal{V}_{n}(\mathcal{I})_{p}$ se e somente se a $F^{\alpha}(\widetilde{E})=$ 0 . De (3.12) temos que $\mathcal{V}_{n}(\mathcal{I})$ é definido por equações que são polinomiais em $p_{i}^{a}$ e os coeficientes são funções em $\Sigma$.

Definição 3.3.1. Seja $\rho: G_{n}(T \Sigma) \rightarrow \Sigma$ a projeção. Definimos a codimensão de $\mathcal{V}_{n}(\mathcal{I})$ em $E$, denotada por $\operatorname{codim}_{E}\left(\mathcal{V}_{n}(\mathcal{I})\right)$, o número máximo de funções $F^{\alpha}$ em $G_{n}\left(T_{\rho(E)} \Sigma\right)$ que se anulam em $\mathcal{V}_{n}(\mathcal{I})_{\rho(E)}$ e possuem diferenciais linearmente independentes em $E$.

Definição 3.3.2. Um elemento integral $E \in \mathcal{V}_{n}(\mathcal{I})$ é dito Kähler-ordinário se $\mathcal{V}_{n}(\mathcal{I})$ é subvariedade de $G_{n}(\Sigma)$ em torno de $E$.

Exemplo 3.3.3. Sejam $(I, J)$ sistema Pfaffian linear em $\Sigma$ e $p \in \Sigma$, considere $\left\{\theta^{1}, \cdots, \theta^{s}\right\}$ geradores de $\mathcal{I}$ e $J=\left\{\omega^{1}, \cdots, \omega^{n}\right\}$. Portanto a condição de independência é $\omega^{1} \wedge \cdots \wedge \omega^{n}$. Complete $\left\{\theta^{a}, \omega^{i}\right\}$ a um correferencial de $T_{p} \Sigma$ com formas $\pi^{\epsilon}, 1 \leq \epsilon \leq r$. Do fato que $(I, J)$ é linear de Pfaff segue

$$
d \theta^{a} \equiv \pi_{i}^{a} \wedge \omega^{i} \bmod I=\left(A_{\epsilon i}^{a} \pi^{\epsilon}+C_{i j}^{a} \omega^{j}\right) \wedge \omega^{i} \bmod I .
$$

Em um $n$-plano $E \subset T_{p} \Sigma$ satisfazendo $\left.\left(\omega^{1} \wedge \cdots \wedge \omega^{n}\right)\right|_{E} \neq 0$, por linearidade temos $\pi^{\epsilon}=p_{i}^{\epsilon} \omega^{i}$. Se $E$ é elemento integral então $\left.\theta^{a}\right|_{E}=0$, logo

$$
\begin{aligned}
0 & =\left.d \theta^{a}\right|_{E} \\
& =\left(A_{\epsilon i}^{a} \pi^{\epsilon}+C_{i j}^{a} \omega^{j}\right) \wedge \omega^{i} \\
& =\left(A_{\epsilon i}^{a}\left(p_{j}^{\epsilon} \omega^{j}\right)+C_{i j}^{a} \omega^{j}\right) \wedge \omega^{i} \\
& =\sum_{i<j}\left(A_{\epsilon i}^{a} p_{j}^{\epsilon}-A_{\epsilon j}^{a} p_{i}^{\epsilon}+C_{i j}^{a}-C_{j i}^{a}\right) \omega^{i} \wedge \omega^{j},
\end{aligned}
$$

portanto para cada $i, j$, e para cada $a$

$$
A_{\epsilon i}^{a} p_{j}^{\epsilon}-A_{\epsilon j}^{a} p_{i}^{\epsilon}+C_{i j}^{a}-C_{j i}^{a}=0
$$

Note que (3.13) é linear em $p_{i}^{\epsilon}$, e se o posto de $F=A_{\epsilon i}^{a} p_{j}^{\epsilon}-A_{\epsilon j}^{a} p_{i}^{\epsilon}+C_{i j}^{a}-C_{j i}^{a}: G_{n}\left(T_{p} \Sigma\right) \rightarrow \mathbb{R}$ é localmente constante (igual a 1 ), então $F^{-1}(0)=\mathcal{V}_{n}(\mathcal{I})_{p}$ é subvariedade.

Exemplo 3.3.4. Sejam $\Sigma=\mathbb{R}^{2}, \theta=y^{2} d x-x d y$ e $\mathcal{I}=\{\theta\}_{\text {diff }}$. Vamos calcular $\mathcal{V}_{1}(\mathcal{I})$. Se $(x, y) \neq 0$, então $x$ ou $y$ é não nulo, suponha $x \neq 0$ e a reta $r(t)=t\left(v^{1}, v^{2}\right)$ em $\mathcal{V}_{1}$. Então $\theta_{(x, y)}\left(t\left(v^{1}, v^{2}\right)\right)=0, \forall t \in \mathbb{R}$, isto é, $t\left(y^{2} v^{1}-x v^{2}\right)=0, \forall t \in \mathbb{R}, \operatorname{logo} y^{2} v^{1}-x v^{2}=0$. 
Podemos supor a norma de $v=\left(v^{1}, v^{2}\right)$ igual a 1 , portanto $1=\left(v^{1}\right)^{2}+\frac{y^{4}}{x^{2}}\left(v^{1}\right)^{2}$; isolando $v^{1}$ e $v^{2}$

$$
v^{1}=\frac{1}{\sqrt{1+\frac{y^{4}}{x^{2}}}} \text { e } v^{2}=\frac{y^{2}}{x \sqrt{1+\frac{y^{4}}{x^{2}}}} .
$$

Concluímos que para $x \neq 0$ existe apenas a reta com direção $\left(x, y^{2}\right)$ em $\mathcal{V}_{1}(\mathcal{I})_{(x, y)}$, o raciocínio é inteiramente análogo para o caso $y \neq 0$. Resta analisar a origem. Na origem $\theta$ é identicamente nula, então $\mathcal{V}_{1}(\mathcal{I})_{(0,0)}=G_{1}\left(T_{(0,0)} \mathbb{R}^{2}\right)=\mathbb{R} \mathbb{P}^{1}$. Como em (3.11) podemos introduzir coordenadas locais em $G_{1}\left(T_{(0,0)} \mathbb{R}^{2}\right)$ tais que $\left.d y\right|_{E}=\left.p d x\right|_{E}$, ou seja, $p$ é o coeficiente angular da reta $E$. Logo $0=\left.\theta\right|_{E}=\left.y^{2} d x\right|_{E}-\left.x p d x\right|_{E}$, o que implica que $y^{2}-x p=0$ define $\mathcal{V}_{1}(\mathcal{I})$. Defina $F(x, y, p)=y^{2}-x p$, função definida em um aberto de $G_{1}\left(T \mathbb{R}^{2}\right)$. Então $d F=2 y d y-p d x-x d p$. Em uma reta $E$ pela origem, $d F_{E}=-\left.p(E) d x\right|_{E}$, então $p=0$ é o eixo $x$, que é ponto singular de $F, \operatorname{logo} \mathcal{V}_{1}(\mathcal{I})$ não é subvariedade em torno desse ponto, mas o é para $E$ é tal que $p(E) \neq 0$. Para o eixo $y$, temos que trocar de coordenadas e escrever $\left.d x\right|_{E}=\left.q d y\right|_{E}$, obtemos $0=\left.y^{2} q d y\right|_{E}-\left.x d y\right|_{E}=\left.\left(q y^{2}-x\right) d y\right|_{E}$, e $V_{1}(\mathcal{I})$ é definida por $q y^{2}-x=0$, defina $G(x, y, q)=q y^{2}-x, d G=y^{2} d q+2 y^{2} d y-d x$, então $d G$ no ponto eixo $y$, continuamos denotando por $E$, da origem, é $d G_{E}=-\left.d x\right|_{E} \neq 0$, portanto $\mathcal{V}_{n}(\mathcal{I})$ é subvariedade em torno de $E$.

Definição 3.3.5. Sejam $E \in G_{n}(T \Sigma)$ e $\left\{e_{1}, \cdots, e_{n}\right\}$ base de $E \subset T_{p} \Sigma$. O espaço polar de $E$ é o conjunto

$$
H(E):=\left\{v \in T_{p} \Sigma: \psi\left(v, e_{1}, \cdots, e_{n}\right)=0, \forall \psi \in \mathcal{I}^{n+1}\right\}
$$

Convencionamos chamar de $H(\{0\})$ o espaço dos vetores que anulam as 1 -formas de $\mathcal{I}$.

Proposição 3.3.6. Sejam $E \in \mathcal{V}_{n}(\mathcal{I})$, e $E^{+} \in G_{n+1}\left(T_{p} \Sigma\right)$ tal que $E \subset E^{+}$. Então $H(E)$ é um subespaço vetorial que não depende da escolha de base $\left\{e_{1}, \cdots, e_{n}\right\}$ e satisfaz as seguintes propriedades:

1. $E \subset H(E)$.

2. $E^{+} \subset H(E)$ se, e somente se, $E^{+} \in \mathcal{V}_{n+1}(\mathcal{I})$.

3. Se $v \in H(E)$, então $(v\lrcorner \psi)\left.\right|_{E}=0, \forall \psi \in \mathcal{I}$.

4. Se $E^{+} \in \mathcal{V}_{n+1}(\mathcal{I})$, então $H\left(E^{+}\right) \subset H(E)$. 
5. Se $\left\{\psi^{\alpha}\right\}$ é conjunto gerador algébrico de $\mathcal{I}$, então $v \in H(E)$ se, e somente se $\left.\left(v-\psi^{\alpha}\right)\right|_{E}=0$, para cada gerador $\psi^{\alpha}$ de grau no máximo $n+1$.

Demonstração. Bem definido. Sejam $v \in H\left(E,\left\{e_{1}, \cdots, e_{n}\right\}\right)$ e $\left\{f_{1}, \cdots, f_{n}\right\}$ outra base de $E$. Existe matriz inversível $\left(A_{j}^{i}\right)$ tal que $f_{j}=A_{j}^{i} e_{i}$. Então para cada $\psi \in \mathcal{I}^{n+1}$,

$$
\begin{aligned}
\psi\left(v, f_{1}, \cdots, f_{n}\right) & =\psi\left(v, A_{1}^{i_{1}} e_{i_{1}}, \cdots, A_{n}^{i_{n}} e_{i_{n}}\right) \\
& =A_{1}^{i_{1}} \cdots A_{n}^{i_{n}} \psi\left(v, e_{i_{1}}, \cdots, e_{i_{n}}\right) \\
& =\sum_{i_{1}, \cdots, i_{n}} A_{1}^{i_{1}} \cdots A_{n}^{i_{n}} \operatorname{sgn}\left(i_{1} \cdots i_{n}\right) \underbrace{\psi\left(v, e_{1}, \cdots, e_{n}\right)}_{0} \\
& =0,
\end{aligned}
$$

$\operatorname{logo} v \in H\left(E,\left\{f_{1}, \cdots, f_{n}\right\}\right)$, como $v$ e $\psi$ são arbitrários segue

$$
H\left(E,\left\{e_{1}, \cdots, e_{n}\right\}\right) \subset H\left(E,\left\{f_{1}, \cdots, f_{n}\right\}\right)
$$

e a inclusão contrária é análoga.

Linearidade. Seja $v, w \in H(E)$ e $\lambda \in \mathbb{R}$, para qualquer $\psi \in \mathcal{I}^{n+1}$ temos

$$
\begin{aligned}
\psi\left(v+\lambda w, e_{1}, \cdots, e_{n}\right) & =\psi\left(v, e_{1}, \cdots, e_{n}\right)+\lambda \psi\left(w, e_{1}, \cdots, e_{n}\right) \\
& =0+\lambda 0=0
\end{aligned}
$$

$\operatorname{assim} v+\lambda w \in H(E)$, portanto $H(E)$ é subespaço linear de $T_{p} \Sigma$.

Propriedade 1. Seja $v \in E$. Como $E$ é elemento integral, para cada $\psi \in \mathcal{I}^{n+1}$ temos $\left.\psi\right|_{E}=0$. Em particular, $\psi\left(v, e_{1}, \cdots, e_{n}\right)=0$, logo $v \in H(E)$.

Propriedade 2. Seja $v \in T_{p} \Sigma$ tal que $E^{+}=\operatorname{span}\left\{e_{1}, \cdots, e_{n}, v\right\}$, é suficiente mostrar que se $v \in H(E)$, então $E^{+} \in \mathcal{V}_{n+1}(\mathcal{I})$. De fato, dado qualquer $\psi \in \mathcal{I}^{n+1}$, como $v \in H(E)$, $\psi\left(v, e_{1}, \cdots, e_{n}\right)=0$. Como $v, e_{1}, \cdots, e_{n}$ geram $E^{+}$, segue $\left.\psi\right|_{E^{+}}=0$, portanto $E^{+} \in$ $\mathcal{V}_{n+1}(\mathcal{I})$. Reciprocamente, se $E^{+} \in \mathcal{V}_{n+1}(\mathcal{I})$, então $\left.\psi\right|_{E^{+}}=0$, para cada $\psi \in \mathcal{I}^{n+1}$. Dado $v \in E^{+}$, em particular temos $\psi\left(v, e_{1}, \cdots, e_{n}\right)=0$, logo $v \in H(E)$. Como $v$ é qualquer, segue que $E^{+} \subset H(E)$.

Propriedade 3. Seja $v \in H(E)$, e suponha que $\left.(v-\psi)\right|_{E} \neq 0$ para algum $\psi \in \mathcal{I}$, com $\operatorname{deg}(\psi)=k \leq n$. Tome $\alpha \in \Omega^{n+1-k}(\Sigma)$ tal que

$$
\left.((v-\psi) \wedge \alpha)\right|_{E} \neq 0 .
$$

Para o produto interior temos a seguinte fórmula

$$
\left.(v\lrcorner \psi) \wedge \alpha=v\lrcorner(\psi \wedge \alpha)+(-1)^{k+1} \psi \wedge(v\lrcorner \alpha\right) .
$$


Note que $\left.(-1)^{k+1} \psi \wedge(v\lrcorner \alpha\right) \in \mathcal{I}$, já que $\psi \in \mathcal{I}$. Então do fato de $E$ ser elemento integral, segue $\left.\left((-1)^{k+1} \psi \wedge(v\lrcorner \alpha\right)\right)\left.\right|_{E}=0$. Sejam $v_{1}, \cdots, v_{n} \in E$ quaisquer. Temos $(v\lrcorner(\psi \wedge \alpha))\left(v_{1}, \cdots, v_{n}\right)=(\psi \wedge \alpha)\left(v, v_{1}, \cdots, v_{n}\right)$, mas $\psi \wedge \alpha \in \mathcal{I}^{n+1}$ e $v \in H(E)$, então $(\psi \wedge \alpha)\left(v, v_{1}, \cdots, v_{n}\right)=0$, isto é $\left.(v\lrcorner(\psi \wedge \alpha)\right)\left.\right|_{E}=0$, por $(3.15)$ temos $\left.((v\lrcorner \psi) \wedge \alpha\right)\left.\right|_{E}=0$, contradição com (3.14).

Propriedade 4. Suponha $E^{+} \in \mathcal{V}_{n+1}(\mathcal{I})$ e seja $v \in H\left(E^{+}\right)$. Como $E^{+}$é elemento integral podemos aplicar a Propriedade 3. Assim $(v\lrcorner \psi)\left.\right|_{E^{+}}=0, \forall \psi \in \mathcal{I}$. Em particular para $\psi \in \mathcal{I}^{n+1}, v_{1}, \cdots, v_{n} \in E^{+} \operatorname{temos} \psi\left(v, v_{1}, \cdots, v_{n}\right)=0$. Tome $v_{i}=e_{i}, 1 \leq i \leq n$. Temos $\psi\left(v, e_{1}, \cdots, e_{n}\right)=0$ para cada $\psi \in \mathcal{I}^{n+1}$, ou seja, $v \in H(E)$.

Propriedade 5. Temos que provar apenas a recíproca, pois o outro sentido é assegurado pela Propriedade 3. Suponha que $\left.(v\lrcorner \psi^{\alpha}\right)=0$, para os geradores algébricos de grau no máximo $n+1$. Dado $\psi \in \mathcal{I}^{n+1}$, existem $\beta_{\alpha} \in \Omega^{n+1-k}$ tais que $\psi=\beta_{\alpha} \wedge \psi^{\alpha}$, em que $k=\operatorname{deg}\left(\psi^{\alpha}\right) \leq n+1$. Então

$$
\left.\left.v\lrcorner \psi=\left((v\lrcorner \beta_{\alpha}\right) \wedge \psi^{\alpha}\right)+(-1)^{n+1-k}\left(\beta_{\alpha} \wedge(v\lrcorner \psi_{\alpha}\right)\right) .
$$

Observe que $\left(v-\beta_{\alpha}\right) \wedge \psi^{\alpha} \in \mathcal{I}$, uma vez que $\psi^{\alpha} \in \mathcal{I}$. Como $E$ é elemento integral, temos $\left.\left((v\lrcorner \beta_{\alpha}\right) \wedge \psi^{\alpha}\right)\left.\right|_{E}=0$. Por hipótese $\left.(v\lrcorner \psi^{\alpha}\right)\left.\right|_{E}=0$, então $\left.(-1)^{n+1-k}\left(\beta_{\alpha} \wedge\left(v-\psi_{\alpha}\right)\right)\right|_{E}=0$. Por (3.16) segue $\left.(v-\psi)\right|_{E}=0$, portanto $v \in H(E)$.

Exemplo 3.3.7. Sejam $\theta^{1}, \theta^{2}, \omega^{1}, \omega^{2}, \pi \in \Omega^{1}\left(\Sigma^{5}\right)$, considere $\mathcal{I}=\left\{\theta^{1}, \theta^{2}\right\}_{\text {diff }}$, suponha que essas formas formam um correferencial e sejam válidas

$$
\left\{\begin{array}{l}
d \theta^{1} \equiv \omega^{1} \wedge \pi \bmod \left\{\theta^{1}, \theta^{2}\right\} \\
d \theta^{2} \equiv \omega^{2} \wedge \pi \bmod \left\{\theta^{1}, \theta^{2}\right\}
\end{array}\right.
$$

em um ponto $p \in \Sigma$. Denote $E=\left\{\theta^{1}, \theta^{2}, \omega^{2}, \pi\right\}^{\perp}$, ou seja, a reta anulada simultaneamente por $\theta^{1}, \theta^{2}, \omega^{2}$ e $\pi$, considere $e \in E$ tal que $\omega^{1}(e)=1$. De (3.17) temos que $\mathcal{I}^{2}$ é gerado como espaço vetorial pelo seguinte conjunto

$$
\left\{\omega^{1} \wedge \pi, \omega^{2} \wedge \pi, \theta^{1} \wedge \theta^{2}, \theta^{1} \wedge \omega^{1}, \theta^{1} \wedge \omega^{2}, \theta^{1} \wedge \pi, \theta^{2} \wedge \omega^{1}, \theta^{2} \wedge \omega^{2}, \theta^{2} \wedge \pi\right\}
$$

Suponha $v \in H(E)$. Então $\psi(v, e)=0, \forall \psi \in \mathcal{I}^{2}$. Dividimos em casos. Suponha $\psi=\theta^{1} \wedge \varphi$, em que $\varphi$ é qualquer uma das formas que formam o correferencial, exceto a própria $\theta^{1}$. Então $0=\psi(v, e)=\left(\theta^{1} \wedge \varphi\right)(v, e)=\theta^{1}(v) \varphi(e)-\theta^{1}(e) \varphi(v)$, e a igualdade só não é trivial para $\varphi=\omega^{1}$, nesse caso $0=\theta^{1}(v)$. Repetindo o processo para $\theta^{2}$ 
obtemos o mesmo resultado $\theta^{2}(v)=0$. Resta calcular $\left.v\right\lrcorner\left(\omega^{1} \wedge \pi\right)$ e $\left.v\right\lrcorner\left(\omega^{2} \wedge \pi\right)$. Para o primeiro caso, $0=v\lrcorner\left(\omega^{1} \wedge \pi\right)(e)=\left(\omega^{1} \wedge \pi\right)(v, e)=-\pi(v)$, para o segundo $0=v\lrcorner\left(\omega^{2} \wedge \pi\right)(e)=\left(\omega^{2} \wedge \pi\right)(v, e)=0$, isto é, a única informação acerca de $v$ vem apenas da primeira equação. Concluímos que $v \in H(E)$ se, e somente se, $\theta^{1}(v)=\theta^{2}(v)=$ $\pi(v)=0$, isto é, $H(E)=\left\{\theta^{1}, \theta^{2}, \pi\right\}^{\perp}$, portanto $E$ está contida em um único plano integral $E^{+}=H(E)$.

Consideramos agora $E=\left\{\theta^{1}, \theta^{2}, \omega^{1}, \omega^{2}\right\}^{\perp}$. Novamente temos $\theta^{1}(v)=\theta^{2}(v)=0$, entretanto se $e \in E$ é tal que $\pi(e)=1$, então

$$
\left\{\begin{array}{l}
\left(\omega^{1} \wedge \pi\right)(v, e)=\omega^{1}(v)=0 \\
\left(\omega^{2} \wedge \pi\right)(v, e)=\omega^{2}(v)=0
\end{array}\right.
$$

obtemos $H(E)=E$, assim $E$ não está contido em nenhum elemento integral de dimensão maior.

Exemplo 3.3.8. Como no exemplo anterior, considere o correferencial formado pelas formas $\theta^{1}, \theta^{2}, \omega^{1}, \omega^{2}, \pi^{1}, \pi^{2} \in \Omega^{1}\left(\Sigma^{6}\right)$. Denote $\mathcal{I}=\left\{\theta^{1}, \theta^{2}\right\}_{\text {diff }}$ e suponha

$$
\left\{\begin{array}{l}
d \theta^{1} \equiv \omega^{1} \wedge \pi^{1}+\omega^{2} \wedge \pi^{2} \bmod \left\{\theta^{1}, \theta^{2}\right\} \\
d \theta^{2} \equiv \omega^{1} \wedge \pi^{2} \bmod \left\{\theta^{1}, \theta^{2}\right\}
\end{array}\right.
$$

Seja a reta $E=\left\{\theta^{1}, \theta^{2}, \omega^{1}, \pi^{1}, \pi^{2}\right\}^{\perp}$, e considere $e \in E$ tal que $\omega^{2}(e)=1$. Vamos calcular $H(E)$. Seja $v \in H(E)$. Novamente $\theta^{1}(v)=\theta^{2}(v)=0$. De (3.18) e pelo item 5 da Proposição 3.3.6 basta verificar as relações de

$$
\left\{\begin{array}{l}
v\lrcorner\left(\omega^{1} \wedge \pi^{1}+\omega^{2} \wedge \pi^{2}\right)=0, \\
v\lrcorner \omega^{1} \wedge \pi^{2}=0 .
\end{array}\right.
$$

A segunda equação em $E$ é trivial. A primeira em $E$ torna-se $0=\left(\omega^{1} \wedge \pi^{1}+\omega^{2} \wedge\right.$ $\left.\pi^{2}\right)(v, e)=\omega^{1}(v) \underbrace{\pi^{1}(e)}_{0}-\underbrace{\omega^{1}(e)}_{0} \pi^{1}(v)+\omega^{2}(v) \underbrace{\pi^{2}(e)}_{0}-\underbrace{\omega^{2}(e)}_{1} \pi^{2}(v)=-\pi^{2}(v)$, então $H(E)=$ $\left\{\theta^{1}, \theta^{2}, \pi^{2}\right\}^{\perp}$. Observe que $H(E)$ não é 3-plano integral de $\mathcal{I}$, já que se $e_{1}, f_{1} \in H(E)$ são duais a $\omega^{1}, \pi^{1}$, então

$$
\begin{aligned}
\left(\omega^{1} \wedge \pi^{1}+\omega^{2} \wedge \pi^{2}\right)\left(e_{1}, f_{1}\right) & =\left(\omega^{1} \wedge \pi^{1}\right)\left(e_{1}, f_{1}\right)+\left(\omega^{2} \wedge \pi^{2}\right)\left(e_{1}, f_{1}\right) \\
& =\underbrace{\omega^{1}\left(e_{1}\right)}_{1} \underbrace{\pi^{1}\left(f_{1}\right)}_{1}-\underbrace{\omega^{1}\left(f_{1}\right)}_{0} \underbrace{\pi^{1}\left(e_{1}\right)}_{0}+\omega^{2}\left(e_{1}\right) \underbrace{\pi^{2}\left(f_{1}\right)}_{0}-\omega^{2}\left(f_{1}\right) \underbrace{\pi^{2}\left(e_{1}\right)}_{0} \\
& =1,
\end{aligned}
$$


isto é, $\left.\left(\omega^{1} \wedge \pi^{1}+\omega^{2} \wedge \pi^{2}\right)\right|_{H(E)} \neq 0$.

Definição 3.3.9. Um elemento integral Kähler-ordinário $E$ é dito Kähler-regular se $\operatorname{codim}(H(\widetilde{E}))=\operatorname{codim}(H(E))$, para cada $\widetilde{E}$ em uma vizinhança de $E \in \mathcal{V}_{n}(\mathcal{I})$.

Lema 3.3.10. Sejam $E \in V_{n-1}(\mathcal{I})_{p}$ e $E^{+} \in \mathcal{V}_{n}(\mathcal{I})_{p}$ com $E \subset E^{+} \subset T_{p} \Sigma$. Então

$$
\left.\operatorname{codim}_{E}\left(\mathcal{V}_{n+1}(\mathcal{I})\right)+\operatorname{codim}(H(E)) \leq \operatorname{codim}_{E^{+}}\left(\mathcal{V}_{n}(\mathcal{I})\right)\right)
$$

Se E é Kähler-regular, então vale a igualdade.

Demonstração. [Robert L. Bryant, 1991, p. 70-72]

Lema 3.3.11. Sejam $E \in V_{n-1}(\mathcal{I})_{p}, E^{+} \in \mathcal{V}_{n}(\mathcal{I})_{p}$ e $E \subset E^{+} \subset T_{p} \Sigma$. Assuma E Kählerregular, sejam $\phi^{\nu} \in \mathcal{I}^{n-1}$, com $1 \leq \nu \leq \operatorname{codim}_{E}\left(\mathcal{V}_{n}(\mathcal{I})\right)$, tais que as correspondentes funções $F^{\nu}$ em $G_{n-1}(T \Sigma)$ têm diferenciais linearmente independentes em E. Seja $\left\{\Phi^{\alpha} \in\right.$ $\left.\mathcal{I}^{n}: \alpha \in A\right\}$ conjunto linearmente independente tal que

$$
H(E)=\left\{v \in T_{p} \Sigma:\left.\left(v-\Phi^{\alpha}\right)\right|_{E}=0, \forall \alpha \in A\right\}
$$

Então existe uma 1-forma $\theta$ e uma vizinhança de $U^{+}$de $E^{+}$em $G_{n}(T \Sigma)$ tal que, para cada $\widetilde{E}^{+} \in U^{+}, \widetilde{E}^{+}$é elemento integral e Kähler-ordinário se e somente se $\left.\Phi^{\alpha}\right|_{\widetilde{E}^{+}}=0$ e $\left.\phi^{\nu} \wedge \theta\right|_{\widetilde{E^{+}}}=0$, para cada $\alpha \in A$ e $\nu \in\left\{1, \cdots, \operatorname{codim}_{E}\left(\mathcal{V}_{n}(\mathcal{I})\right)\right\}$.

Demonstração. Seja $s=\operatorname{dim}(\Sigma)-n$, tome um sistema de coordenadas $\left(x_{1}, \cdots, x^{n}\right.$, $\left.y^{1}, \cdots, y^{s}\right)$ em torno de $p$ tal que $E^{+}=\operatorname{span}\left\{\frac{\partial}{\partial x^{1}}, \cdots, \frac{\partial}{\partial x^{n}}\right\},\left.d x^{n}\right|_{E}=0$ e $H(E)$ anulado pelas formas $d y^{\alpha}, 1 \leq \alpha \leq \operatorname{codim}(H(E))$.

Defina $\theta=d x^{n}$ e e xiste vizinhança $U^{+}$de $E^{+}$como em (3.11). Para cada $\widetilde{E}^{+}$nessa vizinhança, defina $p_{i}^{a}\left(\widetilde{E}^{+}\right)$impondo

$$
X_{i}=\frac{\partial}{\partial x^{i}}+p_{i}^{a}\left(\widetilde{E}^{+}\right) \frac{\partial}{\partial y^{a}}
$$

base de $\widetilde{E}^{+}$, com $1 \leq i \leq n$ e $1 \leq a \leq s$. Como $E$ é Kähler-ordinário, então $\phi^{\nu}$ define, localmente, $\mathcal{I}^{n-1}$ e do fato que $E$ é Kähler-regular, existe $U$ vizinhança de $E$ em $G_{n-1}(T \Sigma)$ tal que

$$
\left.H(\widetilde{E})=\left.\left\{v \in T_{p} \Sigma:(v\lrcorner \Phi^{\alpha}\right)\right|_{\widetilde{E}}=0, \forall \alpha \in A\right\}
$$


para $\widetilde{E} \in U \cap \mathcal{V}_{n-1}(\mathcal{I})$. Se $\left.\phi^{\nu} \wedge \theta\right|_{\widetilde{E}^{+}}=0$, então

$$
\begin{aligned}
0 & =\left.\phi^{\nu} \wedge \theta\right|_{\widetilde{E}^{+}}\left(X_{1}, \cdots, X_{n}\right) \\
& =\frac{1}{(n-1) !} \sum_{\sigma \in S_{n}} \operatorname{sgn}(\sigma) \phi^{\nu}\left(X_{\sigma(1)}, \cdots, X_{\sigma(n-1)}\right) \underbrace{d x^{n}\left(X_{\sigma(n)}\right)}_{\delta_{\sigma(n)}^{n}} \\
& =\frac{1}{(n-1) !} \sum_{\sigma \in S_{n}, \sigma(n)=n} \operatorname{sgn}(\sigma) \phi^{\nu}\left(X_{\sigma(1)}, \cdots, X_{\sigma(n-1)}\right) \\
& =\phi^{\nu}\left(X_{1}, \cdots, X_{n-1}\right) .
\end{aligned}
$$

Logo $\widetilde{E} \subset \widetilde{E}^{+}$gerado por $X_{1}, \cdots, X_{n-1}$ é $(n-1)$-plano integral e, se $\left.\Phi^{\alpha}\right|_{\widetilde{E}^{+}}=0$, então para cada $v \in \widetilde{E}^{+} \Phi^{\alpha}\left(v, X_{1}, \cdots, X_{n}\right)=0$. Logo $v \in H(\widetilde{E})$, isto é, $\widetilde{E}^{+} \subset H(\widetilde{E})$. Pelo item 4 da Proposição 3.3.6, $\widetilde{E}^{+} \in \mathcal{V}_{n}(\mathcal{I})$. Portanto $\widetilde{E}^{+}$é elemento integral, resta mostrar que é Kähler-ordinário. De fato, de (3.9) temos que $\mathcal{V}_{n}(\mathcal{I})$ é subvariedade em torno de $E^{+}$definida por $F^{\nu}$ dada pelas $\phi^{\nu}$ e outras $G^{\mu}$, em que $1 \leq \mu \leq \operatorname{codim}(H(E))$. Como $\operatorname{codim}\left(H\left(\widetilde{E}^{+}\right)\right)$é constante em $U$, segue que $\mathcal{V}_{n}(\mathcal{I})$ também é subvariedade em torno de $\widetilde{E}^{+}, \operatorname{logo} \widetilde{E}^{+}$é Kähler-ordinário.

A reciproca é imediata, uma vez que $\Phi^{\alpha} \in \mathcal{I}$ e $\phi^{\nu} \in \mathcal{I}$, logo $\phi^{\nu} \wedge \theta \in \mathcal{I}$, e do fato que como $\widetilde{E}^{+} \in \mathcal{V}_{n}(\mathcal{I})$, temos $\left.\Phi^{\alpha}\right|_{\widetilde{E}^{+}}=0$ e $\left.\left(\phi^{\nu} \wedge \theta\right)\right|_{\widetilde{E}^{+}}=0$.

Com o que desenvolvemos até aqui, podemos passar a uma discussão da versão geral do Teorema de Cartan-Kähler. O Teorema de Cauchy-Kowalevski tem papel fundamental na construção dos resultados a seguir. Dado que esse teorema é verdade apenas para funções analíticas (vide [Lipman Bers, 1971, p. 45-47]), vamos nos restringir à variedades e ideais $\mathcal{I}$ analíticos.

Teorema 3.3.12. (Cartan-Kähler $1^{\underline{a}}$ versão). Sejam $\mathcal{I}$ sistema um diferencial exterior em $\Sigma$ e $P^{n} \subset \Sigma$ uma subvariedade analítica cujos espaços tangentes são elementos integrais de $\mathcal{I}$ e Kähler-regulares em cada $p \in P$. Suponha ainda que cada $H\left(T_{p} P\right)$ têm dimensão $n+1$. Então, para cada $p \in P$, existem uma vizinhança aberta $U \subset \Sigma$ de $p$ e uma única variedade integral analítica $N^{n+1} \subset U$ que contém $P \cap U$.

Esboço da prova. Seja $s=\operatorname{dim}(\Sigma)-\operatorname{dim}(P)$. Seja $\left(x^{0}, x^{1}, \cdots, x^{n}, y^{1}, \cdots, y^{s}\right)$ um sistema de coordenadas analíticas em torno de $p$ tal que $\frac{\partial}{\partial x^{1}}, \cdots, \frac{\partial}{\partial x^{n}}$ geram $T_{p} P$ e $\frac{\partial}{\partial x^{0}} \in$ $H\left(T_{p} P\right)$. Considere $\left\{\phi^{\nu}\right\}_{\nu \in A}$ conjunto gerador de $\mathcal{I}^{n}$ em torno de $p$ e $\left\{\Phi^{\alpha} \in \mathcal{I}^{n+1}: 1 \leq\right.$ $\alpha \leq s\}$ conjunto gerador das equações polares em uma vizinhança de $T_{p} P$. 
Se $N^{n+1}$ é variedade integral contendo $P$, então pelo item 2 da Proposição 3.3.6 temos $T_{p} N \subset H\left(T_{p} P\right)$, ambos têm dimensão $n+1$, portanto segue a igualdade e $\left(x^{0}, x^{1}, \cdots, x^{n}\right)$ serve de sistema de coordenadas de $N$. Suponha $N$ definida por $y^{\alpha}=F^{\alpha}\left(x^{0}, \cdots, x^{n}\right)$. Então $d y^{\alpha}=\sum_{i=0}^{n} \frac{\partial F^{\alpha}}{\partial x^{i}} d x^{i}$ e $X_{i}=\frac{\partial}{\partial x^{i}}+\frac{\partial F^{\alpha}}{\partial x^{i}} \frac{\partial}{\partial y^{\alpha}}$ formam uma base de $T_{p} N$.

Construímos $N$ com o teorema de Cauchy-Kowalevski.

Como em (3.11), em torno de $T_{p} P$ existe aberto $U$ tal que $d y^{\alpha}=p_{i}^{\alpha} d x^{i}$. Defina as funções $A_{\beta}^{\alpha}\left(x^{i}, y^{\gamma}, p_{j}^{\delta}\right)$ e $B\left(x^{i}, y^{\alpha}, p_{j}^{\delta}\right)$ pelas equações em $q^{\beta} \in \mathbb{R}$

$$
\Phi^{\alpha}\left(\frac{\partial}{\partial x^{0}}+q^{\beta} \frac{\partial}{\partial y^{\beta}}, \frac{\partial}{\partial x^{1}}+p_{1}^{\beta} \frac{\partial}{\partial y^{\beta}}, \cdots, \frac{\partial}{\partial x^{n}}+p_{n}^{\beta} \frac{\partial}{\partial y^{\beta}}\right)=A_{\beta}^{\alpha} q^{\beta}+B^{\alpha} .
$$

Como $p_{i}^{\beta}\left(T_{p} P\right)=0$ temos

$$
\begin{aligned}
\Phi^{\alpha}\left(\frac{\partial}{\partial x^{0}}+q^{\beta} \frac{\partial}{\partial y^{\beta}}, \frac{\partial}{\partial x^{1}}, \cdots, \frac{\partial}{\partial x^{n}}\right) & =\Phi^{\alpha}\left(\frac{\partial}{\partial x^{0}}, \frac{\partial}{\partial x^{1}}, \cdots, \frac{\partial}{\partial x^{n}}\right) \\
& +\Phi^{\alpha}\left(\frac{\partial}{\partial y^{\beta}}, \frac{\partial}{\partial x^{1}}, \cdots, \frac{\partial}{\partial x^{n}}\right) q^{\beta}
\end{aligned}
$$

e como $\frac{\partial}{\partial x^{0}} \in H\left(T_{p} P\right)$, o primeiro termo é nulo. Assim para $p_{i}^{\beta}\left(T_{p} P\right)=0$ obtemos

$$
A_{\beta}^{\alpha}=\Phi^{\alpha}\left(\frac{\partial}{\partial y^{\beta}}, \frac{\partial}{\partial x^{1}}, \cdots, \frac{\partial}{\partial x^{n}}\right)
$$

entradas de uma matriz inversível, então diminuindo $U$ se necessário e para $\left\|p_{j}^{\beta}\right\|$ suficientemente pequeno temos $\widetilde{\Phi}^{\alpha}=\left(A^{-1}\right)_{\beta}^{\alpha} \Phi^{\beta}$. Seja $C\left(x^{i}, y^{\alpha}, p_{j}^{\delta}\right)$ definido por

$$
\widetilde{\Phi}^{\alpha}\left(\frac{\partial}{\partial x^{0}}+q^{\beta} \frac{\partial}{\partial y^{\beta}}, \frac{\partial}{\partial x^{1}}+p_{1}^{\beta} \frac{\partial}{\partial y^{\beta}}, \cdots, \frac{\partial}{\partial x^{n}}+p_{n}^{\beta} \frac{\partial}{\partial y^{\beta}}\right)=q^{\alpha}-C^{\alpha},
$$

Construímos $N$ usando Cauchy-Kowalevski para o seguinte sistema

$$
\left\{\begin{array}{l}
\frac{\partial F}{\partial x^{0}}=C^{\alpha}\left(x^{0}, x^{1}, \cdots, x^{n}, F^{1}, \cdots, F^{s}, \frac{\partial F^{\beta}}{\partial x^{j}}\right), \\
F^{\alpha}\left(0, x^{1}, \cdots, x^{n}\right)=0
\end{array}\right.
$$

Teorema de Cauchy-Kowalevski. Denote $x^{0}$ coordenadas em $\mathbb{R},\left(x^{1}, \cdots, x^{n}\right)$ coordenada em $\mathbb{R}^{n}$ e $\left(y^{1}, \cdots, y^{s}\right)$ coordenadas em $\mathbb{R}^{s}$. Sejam $\mathcal{D} \subset \mathbb{R} \times \mathbb{R}^{n} \times \mathbb{R}^{s} \times \mathbb{R}^{n s}$ aberto, $G: \mathcal{D} \rightarrow \mathbb{R}^{s}$ função analítica, $\mathcal{D}_{0} \subset \mathbb{R}^{n}$ aberto e $f: \mathcal{D}_{0} \rightarrow \mathbb{R}^{s}$ função analítica tais que $\Gamma_{f}=\left\{\left(x^{0}, x, f(x), D f(x)\right): x \in \mathcal{D}_{0}\right\} \subset \mathcal{D}$, para algum $x^{0} \in \mathbb{R}$ fixo. Então existem um aberto $\mathcal{D}_{1} \subset \mathbb{R} \times \mathcal{D}_{0}$ que contém $\left\{x^{0}\right\} \times \mathcal{D}_{0}$ e uma aplicação $F: \mathcal{D}_{1} \rightarrow \mathbb{R}^{s}$ solução de

$$
\left\{\begin{array}{l}
\frac{\partial F^{\alpha}}{\partial x^{0}}=G\left(x^{0}, x, F(x), \frac{\partial F}{\partial x}\right) \\
F\left(x^{0}, x\right)=f(x), \forall x \in \mathcal{D}_{0}
\end{array}\right.
$$


Seja $H$ qualquer outra solução da equação acima. Então $H$ satisfaz $F \equiv H$ em um aberto que contém $\left\{x_{0}\right\} \times \mathcal{D}_{0}$.

Para $x^{0}=0$ e $f \equiv 0$, construímos

$$
N^{n+1}=\left\{\left(x^{0}, x^{1}, \cdots, x^{n}, F^{1}\left(x^{0}, x^{1}, \cdots, x^{n}\right), \cdots, F^{s}\left(x^{0}, \cdots, x^{n}\right)\right):\left(x^{0}, \cdots, x^{1}\right) \in V\right\},
$$

em que possivelmente tenhamos que diminuir o domínio do sistema de coordenadas. Resta mostrar que $N$ é variedade integral. É suficiente mostrar que o pull-back de cada $\Psi \in$ $\mathcal{I}^{n+1}$ é zero. Pelo Lema 3.3.11, basta verificar para $\phi^{\nu} \wedge d x^{0}$, o que é equivalente a $\phi^{\nu}\left(X_{1}, \cdots, X_{n}\right)=0$. Para $X_{i}$ base de $T_{p} N$ temos

$$
\frac{\partial}{\partial x^{0}} \phi^{\nu}\left(X_{1}, \cdots, X_{n}\right)=d \phi^{\nu}\left(X_{0}, X_{1}, \cdots, X_{n}\right)-\sum_{i=1}^{n} \frac{\partial}{\partial x^{i}}\left(\left(d x^{i} \wedge \phi^{\nu}\right)\left(X_{0}, X_{1}, \cdots, X_{n}\right)\right) .
$$

Como $d \phi^{\nu}$ e $\phi^{\nu} \wedge d x^{i}$ são $0 \bmod \left\{\Phi^{\alpha}, \phi^{\nu} \wedge d x^{0}\right\}$, o lado direito da EDP acima é expressa em termos de funções analíticas e suas $x^{i}$-derivadas. Para $x^{0}=0$ temos $\phi^{\nu}\left(X_{1}, \cdots, X_{n}\right)=0$, e pela unicidade do Teorema de Cauchy-Kowalevski segue que

$$
\phi^{\nu}\left(X_{1}, \cdots, X_{n}\right)=0
$$

para cada $\nu \in A$, portanto $N$ é variedade integral que contém $P \cap U$.

Segue do resultado acima duas versões extremamente úteis do Teorema de CartanKähler.

Corolário 3.3.13. (Cartan-Kähler $2^{a}$ versão). Assuma $\Sigma, I$ e $P^{n}$ com as mesmas propriedades da $1^{\underline{a}}$ versão, mudando apenas $H\left(T_{p} P\right)$ com dimensão $n+r+1$ para cada $p \in P$. Suponha $R \subset \Sigma$ subvariedade analítica de codimensão $r$ tal que $P \subset R$ e $T_{p} R$ é transversal a $H\left(T_{p} P\right)$. Então, para cada $p \in P$, existe uma vizinhança $U \subset R$ de $p$ e uma única variedade integral $N^{n+1} \subset U$ que contém $P \cap U$.

Corolário 3.3.14. (Cartan-Kähler $3^{\underline{a}}$ versão). Sejam $E_{k}, 0 \leq k \leq n$, cadeia de elementos integrais em $p$ de um sistema diferencial exterior analítico, com $\operatorname{dim}\left(E_{k}\right)=k$ tal que $E_{k}$ é Kähler-regular para $0 \leq k \leq n-1$. Então existe uma variedade integral $N^{n}$ cujo espaço tangente em $p$ é $E_{n}$. Ainda mais, seja $c_{k}=\operatorname{codim}(H(E))$, para $0 \leq k \leq n$, e seja $\left(x^{1}, \cdots, x^{n}, y^{1}, \cdots, y^{s}\right)$ sistema de coordenadas de $\Sigma$, em torno de $p$, tal que $E_{k}=\operatorname{span}\left\{\frac{\partial}{\partial x^{1}}, \cdots, \frac{\partial}{\partial x^{k}}\right\}$ e $H(E)$ anulado por $d y^{1}, \cdots, d y^{c_{k}}$. Se $N$ é definida nessas coordenadas pelas equações $y^{a}=F^{a}\left(x^{1}, \cdots, x^{n}\right), 1 \leq a \leq s$, então $N$ é unicamente 
determinada determinada pelo sistema

$$
\begin{cases}f^{a}\left(x^{1}\right)=F^{a}\left(x^{1}, 0, \cdots, 0\right) & \text { para } c_{0} \leq a \leq c_{1}, \\ f^{a}\left(x^{1}, x^{2}\right)=F^{a}\left(x^{1}, x^{2}, 0, \cdots, 0\right) & \text { para } c_{1} \leq c_{2}, \\ \cdots & \\ f^{a}\left(x^{1}, \cdots, x^{n-1}\right)=F^{a}\left(x^{1}, \cdots, x^{n-1}, 0\right) & \text { para } c_{n-2} \leq a \leq c_{n-1} .\end{cases}
$$

Reciprocamente, existe $N$ correspondente ao sistema acima se $f^{a}$ garantir a transversalidade da $2^{\underline{a}}$ versão.

Observe que para aplicar alguma das versões do Cartan-Kähler, temos a difícil tarefa de calcular espaços polares de uma vizinhança de um elemento integral. O próximo resultado nos dá um teste para evitar esse cálculo.

Teorema 3.3.15. (Teste de Cartan). Sejam $E_{k}, 0 \leq k \leq n$, cadeia de elementos integrais de $\mathcal{I}$ em $p$, seja $c_{k}=\operatorname{codim}\left(H\left(E_{k}\right)\right)$ para $0 \leq k \leq n-1$. Então

$$
c_{0}+c_{1}+\cdots+c_{n-1} \leq \operatorname{codim}_{E}\left(\mathcal{V}_{n}(\mathcal{I})\right)
$$

Mais ainda, $\mathcal{V}_{n}(\mathcal{I})_{p}$ é subvariedade de codimensão $c_{0}+c_{1}+\cdots+c_{n-1}$ em torno de $E_{n}$ se, e somente se, $E_{k}$ são todos Kähler-regular para $0 \leq k \leq n-1$.

Demonstração. [Robert L. Bryant, 1991, p. 74-77].

Definição 3.3.16. Dizemos que $E$ é um elemento integral ordinário se existe cadeia para $E$ tal que a desigualdade (3.20) é igualdade. Se existe uma vizinhança de $E$ de elementos integrais ordinários, dizemos que $\mathcal{I}$ é involutivo em $E$.

Vamos usar o Teste de Cartan da seguinte forma. Seja $E \in \mathcal{V}_{n}(\mathcal{I})$ Kähler-ordinário, com cadeia $\{0\}=E_{0} \subset E_{1} \subset \cdots \subset E_{n}=E$ fixada. Defina $s_{0}=c_{0}, s_{k}=c_{k}-c_{k-1}$ e $s_{n}=\operatorname{codim}(E)-c_{n-1}$. Dizemos que $s_{k}$ são os caracteres de $\mathcal{I}$, para $1 \leq k \leq n-1$. Por definição $c_{k}=s_{k}+c_{k-1}$, recursivamente,

$$
c_{k}=s_{k}+s_{k-1}+\cdots+s_{0}
$$

Substituindo a igualdade acima em (3.20) obtemos

$$
n s_{0}+(n-1) s_{1}+\cdots+2 s_{n-2}+s_{n-1} \leq \operatorname{codim}_{E}\left(\mathcal{V}_{n}(\mathcal{I})\right) .
$$


Em particular $s_{n}=\operatorname{codim}(E)-\left(s_{n-1}+\cdots+s_{0}\right)$. Como $\operatorname{codim}(E)=\operatorname{dim}(\Sigma)-\operatorname{dim}(E)=$ $\operatorname{dim}(\Sigma)-n$, segue $s_{0}+\cdots+s_{n}=\operatorname{dim}(\Sigma)-n$. Podemos reescrever (3.21) como

$$
\begin{aligned}
\operatorname{codim}_{E}\left(\mathcal{V}_{n}(\mathcal{I})\right) & \geq s_{0}+s_{1}+\cdots+s_{n-1} \\
& +s_{0}+s_{1}+\cdots+s_{n-2} \\
& +\cdots \\
& +s_{0}+s_{1}+\cdots+s_{n-i} \\
& +\cdots \\
& +s_{0} .
\end{aligned}
$$

Em cada linha $i$ podemos somar e subtrair $s_{n-i+1}+\cdots+s_{n}$, e usando a relação $\sum_{i=0}^{n} s_{i}=$ $\operatorname{dim}(\Sigma)-n$, obtemos

$$
\begin{aligned}
\operatorname{codim}_{E}\left(\mathcal{V}_{n}(\mathcal{I})\right) & \geq(\operatorname{dim}(\Sigma)-n)-s_{n} \\
& +(\operatorname{dim}(\Sigma)-n)-s_{n}-s_{n-1} \\
& +\cdots \\
& +(\operatorname{dim}(\Sigma)-n)-s_{n}-s_{n-1}+\cdots s_{n-i+1} \\
& +\cdots \\
& +(\operatorname{dim}(\Sigma)-n)-s_{n}-s_{n-1}+\cdots+s_{1} \\
& =n(\operatorname{dim}(\Sigma)-n)-\left(s_{1}+2 s_{2}+\cdots+n s_{n}\right) .
\end{aligned}
$$

como $\operatorname{dim}\left(G_{n}\left(T_{\rho(E)} \Sigma\right)\right)=n(\operatorname{dim}(\Sigma)-n)$,

$$
\operatorname{dim}\left(G_{n}\left(T_{\rho(E)} \Sigma\right)\right)-\operatorname{codim}_{E}\left(\mathcal{V}_{n}(\mathcal{I})\right) \leq s_{1}+2 s_{2}+\cdots+n s_{n}
$$

$E$ é Kähler-ordinário, então $\mathcal{V}_{n}(\mathcal{I})_{\rho(E)}$ é variedade diferenciável, nesse caso

$$
\operatorname{dim}\left(\mathcal{V}_{n}(\mathcal{I})_{\rho(E)}\right) \leq \operatorname{dim}\left(G_{n}\left(T_{\rho(E)} \Sigma\right)\right)-\operatorname{codim}_{E}\left(\mathcal{V}_{n}(\mathcal{I})\right)
$$

De fato, seja $k=\operatorname{codim}_{E}\left(\mathcal{V}_{n}(\mathcal{I})\right)$. Por definição existem $F^{1}, \cdots, F^{k}: U \subset G_{n}(T \Sigma) \rightarrow \mathbb{R}$ tais que $\mathcal{V}_{n}(\mathcal{I}) \subset \bigcap_{i=1}^{k}\left(F^{k}\right)^{-1}(\{0\})$ e o conjunto $\left\{d F_{E}^{1}, \cdots d F_{E}^{k}\right\}$ é linearmente independente. Defina $F: U \rightarrow \mathbb{R}^{k}$ por $F(\widetilde{E})=\left(F^{1}(\widetilde{E}), \cdots, F^{k}(\widetilde{E})\right)$. Como $d F^{1}, \cdots, d F^{k}$ são linearmente independentes em $E, d F_{E}$ é sobrejetora, então, diminuindo $U$ se necessário, $F$ é submersão. Temos $\mathcal{V}_{n}(\mathcal{I})_{\rho(E)} \subset F^{-1}(\{0\}), F^{-1}(\{0\})$ é subvariedade de dimensão 
$\operatorname{dim}\left(G_{n}\left(T_{\rho(E)} \Sigma\right)\right)-k$, portanto $\operatorname{dim}\left(\mathcal{V}_{n}(\mathcal{I})_{\rho(E)}\right) \leq \operatorname{dim}\left(F^{-1}(\{0\})\right)=\operatorname{dim}\left(G_{n}\left(T_{\rho(E)} \Sigma\right)\right)-$ $\operatorname{codim}_{E}\left(\mathcal{V}_{n}(\mathcal{I})\right)$. Concluímos

$$
\operatorname{dim}\left(\mathcal{V}_{n}(\mathcal{I})_{\rho(E)}\right) \leq s_{1}+2 s_{2}+\cdots+n s_{n}
$$

Observe que se $\mathcal{V}_{n}(\mathcal{I})$ for subfibrado, então $\operatorname{dim}\left(\mathcal{V}_{n}(I)_{\rho(E)}\right)=\operatorname{dim}\left(\mathcal{V}_{n}(\mathcal{I})\right)-\operatorname{dim}(\Sigma)$, e podemos reescrever $(3.23)$ como $\operatorname{dim}\left(\mathcal{V}_{n}(\mathcal{I})\right)-\operatorname{dim}(\Sigma) \leq s_{1}+2 s_{2}+\cdots+n s_{n}$. Aplicando o Teste de Cartan, a igualdade em (3.23) vale se $E$ é Kähler-ordinário e $c_{0}+\cdots+c_{n-1}=$ $\operatorname{codim}\left(\mathcal{V}_{n}(\mathcal{I})_{\rho(E)}\right)$, e somente se, $E_{k}$ são Kähler-regulares para $k=0, \cdots, n-1$ e $E_{k}$ são escolhidos de forma que $\operatorname{dim}\left(E_{k}\right)=k$. Da $3^{\text {a }}$ versão do Teorema de Cartan-Kähler a igualdade em (3.23) garante a existência de variedade integral $N^{n}$ de $\mathcal{I} \operatorname{com} E=T_{\rho(E)} N$.

Observação 3.3.17. Caso linear de Pfaff. Vimos no passo 5 do algoritmo de Cartan que o espaço dos elementos integrais pode ser identificado com $\Sigma \times A^{(1)}$. Então (3.23), nesse caso, torna-se $\operatorname{dim}\left(A^{(1)}\right) \leq s_{1}+2 s_{2}+\cdots+n s_{n}$. Resta mostrar a compatibilidade das definições de $s_{k}$.

Seja $(I, J)$ sistema Pfaffian linear, denote $I=\left\{\theta^{1}, \cdots, \theta^{s}\right\}, J=\left\{\omega^{1}, \cdots, \omega^{n}\right\}$ e $d \theta^{a}=$ $\pi_{i}^{a} \wedge \omega^{i} \bmod I$. Seja $E \in V_{n}(\mathcal{I})$. Podemos supor $\left.\pi_{i}^{a}\right|_{E}=0$; caso não seja, considere $\widetilde{\pi}_{i}^{a}=\pi_{i}^{a}-p_{i j}^{a} \omega^{j}$, em que $p_{i j}^{a}$ é como no passo 5 do algoritmo de Cartan, então $\left.\pi_{i}^{a}\right|_{E}=$ $\left.p_{i j}^{a} \omega^{j}\right|_{E}$, portanto $\left.\widetilde{\pi}_{i}^{a}\right|_{E}=0$. Pela simetrias dos índices em $p_{i j}^{a}$ temos $d \theta^{a}=\widetilde{\pi}_{i}^{a} \wedge \omega^{i}$. Seja $\left\{e_{1}, \cdots, e_{n}\right\}$ base de $E$ dual a $\left\{\omega^{1}, \cdots, \omega^{n}\right\}$, defina $E_{k}=\operatorname{span}\left\{e_{1}, \cdots, e_{k}\right\}$, e obtemos a cadeia $\{0\}=E_{0} \subset E_{1} \subset \cdots \subset E_{n}=E$. Observe que $H\left(E_{0}\right)$ é, por definição, o espaço que anula cada $\theta^{a}$. Aplicando o item 5 da Proposição 3.3.6, $v \in H\left(E_{k}\right)$ se, e somente se $\theta^{a}(v)=0$ e $d \theta^{a}\left(v, e_{i}\right)=0$, para $i \leq k$. Do fato que $d \theta^{a}=\pi_{i}^{a} \wedge \omega^{i} \bmod I$, obtemos

$$
\begin{aligned}
d \theta^{a}\left(v, e_{i}\right) & =\left(\pi_{j}^{a} \wedge \omega^{j}\right)\left(v, e_{i}\right) \\
& =\pi_{j}^{a}(v) \omega^{j}\left(e_{i}\right)-\pi_{i}^{a}\left(e_{j}\right) \underbrace{\omega^{j}(v)}_{0} \\
& =\pi_{j}^{a}(v) \delta_{i}^{j} \\
& =\pi_{i}^{a}(v) .
\end{aligned}
$$

$\operatorname{Logo} v \in H\left(E_{k}\right)$ se, e somente se, $\theta^{a}(v)=0$ e $\pi_{i}^{a}(v)=0$, para $i \leq k$. Da Definição 3.2.14, se $\left\{\pi^{\epsilon}\right\}$ é complemento de $\left\{\theta^{a}, \omega^{i}\right\}$ a um correferencial e supondo torção nula, $\pi_{i}^{a}=A_{\epsilon i}^{a} \pi^{\epsilon}$, e $A$ é gerado por $\left\{\sum_{a=1}^{s} A_{\epsilon i}^{a} \theta^{a} \otimes \omega^{i}: 1 \leq \epsilon \leq \operatorname{dim}(\Sigma)-\operatorname{dim}(J)-\operatorname{dim}(I)\right\}$, então $\left.\pi_{i}^{a}\right|_{H\left(E_{k}\right)}=0$ implica em $\left.A_{\epsilon i}^{a} \pi^{\epsilon}\right|_{H\left(E_{k}\right)}=0$, assim $A_{\epsilon i}^{a}=0$, para $i \leq k$, isto é, a cada espaço polar $H\left(E_{k}\right)$ 
podemos associar o subespaço de $A$

$$
B_{k}:=\operatorname{span}\left\{\sum_{a=1}^{s} B_{\epsilon i}^{a} \theta^{a} \otimes \omega^{i}: 1 \leq \epsilon \leq \operatorname{dim}(\Sigma)-\operatorname{dim}(J)-\operatorname{dim}(I)\right\},
$$

em que

$$
B_{\epsilon i}^{a}:=\left\{\begin{array}{l}
0, \text { se } i \leq k \\
A_{\epsilon i}^{a}, \text { caso contrário. }
\end{array}\right.
$$

Em termos de matrizes, cada matriz de $B_{k}$ têm entradas $(a, i)$ nulas, para $i \leq k$, isto é, $B_{k}$ é o espaço formados pelas matrizes de $A$ com as $k$ primeiras colunas zeradas. Observe que a definição de $B_{k}$ é exatamente a definição $A_{k}$ da Proposição 3.1.7. Denotando momentaneamente $\widetilde{s}_{k}$ os caracteres definidos na seção 3.1, temos $\operatorname{dim}\left(A_{k}\right)=\widetilde{s}_{k+1}+\cdots+\widetilde{s}_{n}$, $\operatorname{logo} \operatorname{codim}\left(A_{k}\right)-\operatorname{codim}\left(A_{k-1}\right)=\widetilde{s}_{k}$. Por outro $\operatorname{lado} \operatorname{codim}\left(H\left(E_{k}\right)\right)-\operatorname{codim}\left(H\left(E_{k-1}\right)\right)=$ $c_{k}-c_{k-1}=s_{k}$, então existe uma correspondência entre $s_{k}$ e $\widetilde{s}_{k}$ para $k=1, \cdots, n-1$. É verdade que $\operatorname{dim}\left(H\left(E_{k}\right)\right)=\operatorname{dim}\left(A_{k}\right)$, a referência para esse fato é [Robert L. Bryant, 1991, p. 121], portanto $s_{k}=\widetilde{s}_{k}$, para $k=1, \cdots, n-1$. Note que $H(E)$ está associado a $A$, mas definimos $s_{n}$ em função $\operatorname{codim}(E)$ e não em função $\operatorname{codim}(H(E))$, ou seja, $s_{n}=\operatorname{codim}(E)-c_{n-1}$.

Seja $\mathcal{A}(\mathcal{I})$ espaço dos vetores característicos de Cauchy. Por definição de vetor característico temos $v\lrcorner \alpha \in \mathcal{I}, \forall \alpha \in \mathcal{I}$, em particular $v\lrcorner \psi \in \mathcal{I}^{n}, \forall \psi \in \mathcal{I}^{n+1}$, se $E$ é elemento integral então $(v\lrcorner \psi)\left.\right|_{E}=0$, ou seja, $v \in H(E)$, isto é $\mathcal{A}(\mathcal{I})_{\rho(E)} \subset H(E)$. Denote $k:=\operatorname{dim}\left(A(\mathcal{I})_{\rho(E)}\right)$, sejam $\{0\}=E_{0} \subset E_{1} \subset \cdots \subset E_{n}=E$ cadeia em $E$ e $Q$ quociente pelas características, vide Definição A.2.10, suponha $Q$ variedade diferenciável e $\tilde{\mathcal{I}}$ ideal dado pelo Teorema A.2.12, denote $\bar{s}_{t}$ caracteres de $\tilde{\mathcal{I}}$ dados por $H\left(E_{t}\right) / \mathcal{A}(\mathcal{I})_{\rho(E)}$. Por definição $\bar{s}_{t}=\bar{c}_{t}-\bar{c}_{t-1}$, portanto

$$
\begin{aligned}
\bar{s}_{t} & =\bar{c}_{t}-\bar{c}_{t-1} \\
& =\left(c_{t}-k\right)-\left(c_{t-1}-k\right) \\
& =c_{t}-c_{t-1} \\
& =s_{t},
\end{aligned}
$$

$\operatorname{assim} \bar{s}_{t}=s_{t}$ para $t=1, \cdots, n-1, \operatorname{agora} \bar{s}_{n}=\operatorname{codim}(E)-\bar{c}_{n-1}=\operatorname{codim}(E)-\left(c_{n-1}-k\right)=$ $s_{n}+k$. Pode-se mostrar que $\operatorname{dim}\left(\mathcal{V}_{n}(\widetilde{\mathcal{I}})_{\pi(\rho(E))}\right)=\operatorname{dim}\left(\mathcal{V}_{n}(\mathcal{I})_{\rho(E)}\right)+n k$, portanto (3.23) em 
$Q$ é

$$
\begin{aligned}
\operatorname{dim}\left(\mathcal{V}_{n}(\mathcal{I})_{\rho(E)}\right)+n k & =\operatorname{dim}\left(\mathcal{V}_{n}(\tilde{\mathcal{I}})_{\pi(\rho(E)}\right) \\
& \leq \bar{s}_{1}+2 \bar{s}_{2}+\cdots+n \bar{s}_{n} \\
& =s_{1}+2 s_{2}+\cdots+(n-1) s_{n-1}+n\left(s_{n}+k\right) \\
& =s_{1}+2 s_{2}+\cdots+n s_{n}+n k,
\end{aligned}
$$

isto é, o Teste de Cartan em $Q$ é equivalente ao mesmo teste em $\Sigma$. A diferença entre $s_{n}$ e $\widetilde{s}_{n}$ no caso Pfaffian linear é justamente o fato de $s_{n}$ contar o espaço dos vetores característicos.

Exemplo 3.3.18. (Superfícies de Weingarten). Uma superfície $M \subset \mathbb{E}^{3}$ é dita Superfície de Weingarten se satisfaz uma equação do tipo $f(K, H)=0$, para $K$ curvatura Gaussiana e $H$ curvatura média. Nesse exemplo vamos tratar o caso particular $f(x, y)=A x+2 B y+$ $C$, para $A, B, C \in \mathbb{R}, A \neq 0$ e $B^{2}-A C \neq 0$.

Denote $\Sigma=A S O(3)$, no Exemplo 1.3 .4 vimos que uma superfície $M \subset \mathbb{E}^{3}$ induz um subfibrado $\mathcal{F}^{1}$ de $\Sigma$ tal que a forma de Maurer-Cartan restrita a esse subfibrado satisfaz

$$
\left\{\begin{array}{l}
\omega_{1}^{3}=h_{11} \omega^{1}+h_{12} \omega^{2} \\
\omega_{2}^{3}=h_{21} \omega^{1}+h_{22} \omega^{2} \\
d \omega_{2}^{1}=\omega_{1}^{3} \wedge \omega_{2}^{3}=K \omega^{1} \wedge \omega^{2} .
\end{array}\right.
$$

Note também que de (3.24) obtemos

$$
\begin{aligned}
\omega_{1}^{3} \wedge \omega^{2}-\omega_{2}^{3} \wedge \omega^{1} & =\left(h_{11} \omega^{1}+h_{12} \omega^{2}\right) \wedge \omega^{2}-\left(h_{21} \omega^{1}+h_{22} \omega^{2}\right) \wedge \omega^{1} \\
& =h_{11} \omega^{1} \wedge \omega^{2}-h_{22} \omega^{2} \wedge \omega^{1} \\
& =\left(h_{11}+h_{22}\right) \omega^{1} \wedge \omega^{2} \\
& =\operatorname{tr}(h) \omega^{1} \wedge \omega^{2} \\
& =2 H \omega^{1} \wedge \omega^{2}
\end{aligned}
$$

Se definirmos $\mathcal{I}=\left\{\omega^{3}, d \omega^{3}, \Theta=A \omega_{1}^{3} \wedge \omega_{2}^{3}+B\left(\omega_{1}^{3} \wedge \omega^{2}-\omega_{2}^{3} \wedge \omega^{1}\right)+C \omega^{1} \wedge \omega^{2}\right\}_{\text {alg }}$ ideal em $\Sigma$, omitindo o pull-back pela inclusão $i: \mathcal{F}^{1} \rightarrow \Sigma$, então $\omega^{3}=0, \operatorname{logo} d \omega^{3}=0$, e

$$
\begin{aligned}
\Theta & =A K \omega^{1} \wedge \omega^{2}+2 B H \omega^{1} \wedge \omega^{2}+C \omega^{1} \wedge \omega^{2} \\
& =f(K, H) \omega^{1} \wedge \omega^{2}
\end{aligned}
$$


portanto $\mathcal{F}^{1}$ é integral de $\mathcal{I}$, com condição de independência $\Omega=\omega^{1} \wedge \omega^{2}$, se, e somente se, $f(K, H)=0$, isto é, as variedades integrais de $\mathcal{I}$ são exatamente os fibrados de referenciais adaptados de superfícies de Weingarten que estamos analisando.

O próximo passo é aplicar o Teste de Cartan para $\mathcal{I}$. Seja $E \in G_{2}(T \Sigma)$ satisfazendo $\left.\omega^{1} \wedge \omega^{2}\right|_{E} \neq 0$. Vimos em $(3.11)$ que $\left.\omega_{i}^{3}\right|_{\widetilde{E}}=\left.h_{i j}(\widetilde{E}) \omega^{j}\right|_{\widetilde{E}}$ para funções $h_{i j}$, simétricas nos índices, definidas em um aberto $U$ em torno de $E$. Um elemento de $v \in \widetilde{E}$ é da forma

$$
\left(\left(\begin{array}{c}
0 \\
\omega^{1}(v) \\
\omega^{2}(v) \\
\omega^{3}(v)
\end{array}\right)\left(\begin{array}{ccc}
0 & \omega_{2}^{1}(v) & \omega_{3}^{1}(v) \\
-\omega_{2}^{1}(v) & 0 & \omega_{3}^{2}(v) \\
-\omega_{3}^{1}(v) & -\omega_{3}^{2}(v) & 0
\end{array}\right)\right)
$$

considere $\left\{\widetilde{e}^{1}, \widetilde{e}^{2}\right\}$ dual a $\left\{\left.\omega^{1}\right|_{\widetilde{E}},\left.\omega^{2}\right|_{\widetilde{E}}\right\}$. Então, para cada $v \in E$, temos $v=t \widetilde{e}^{1}+s \widetilde{e}^{2}$. Escreva $\left.\omega^{3}\right|_{\widetilde{E}}=\left.a \omega^{1}\right|_{\widetilde{E}}+\left.b \omega^{2}\right|_{\widetilde{E}}$, então $v$ torna-se

$$
v=\left(\left(\begin{array}{c}
0 \\
t \\
s \\
a t+b s
\end{array}\right)\left(\begin{array}{ccc}
0 & \omega_{2}^{1}(v) & -h_{11} t-h_{12} s \\
-\omega_{2}^{1}(v) & 0 & -h_{12} t-h_{22} s \\
h_{11} t+h_{12} s & h_{12} t+h_{22} s & 0
\end{array}\right)\right)
$$

isto é, $\widetilde{E}$ fica determinado pela matriz $\left(\begin{array}{ll}h_{11} & h_{12} \\ h_{12} & h_{22}\end{array}\right)$ e pelas funções $a$ e $b$ avaliadas em $\widetilde{E}$, e pelos valores de $\omega_{2}^{1}$.

Afirmação: $\mathcal{A}(\mathcal{I})=\left\{\omega^{1}, \omega^{2}, \omega^{3}, \omega_{3}^{1}, \omega_{3}^{2}\right\}^{\perp}$, isto é, o espaço dos vetores característicos de Cauchy é dual a $\omega_{2}^{1}$.

De fato, seja $v \in \mathcal{A}(\mathcal{I})$, temos que $v\lrcorner \omega^{3}$ é função, $\left.\log o v\right\lrcorner \omega^{3} \in \mathcal{I}$ se, e somente se $v\lrcorner \omega^{3}=0$. Para $\left.v\right\lrcorner d \omega^{3} \in \mathcal{I} \subset \Omega^{1}(\Sigma)$, nesse caso $\left.v\right\lrcorner d \omega^{3}$ deve ser múltiplo de $\omega^{3}$, mas

$$
v-d \omega^{3}=-\omega_{1}^{3}(v) \omega^{1}+\omega^{1}(v) \omega_{1}^{3}-\omega_{2}^{3}(v) \omega^{2}+\omega^{2}(v) \omega_{2}^{3}
$$

como $\left\{\omega^{3}, \omega^{1}, \omega^{2}, \omega_{1}^{3}, \omega_{2}^{3}\right\}$ é conjunto linearmente independente, então $\left.v\right\lrcorner d \omega^{3} \in \mathcal{I}$ se, e somente se $v\lrcorner d \omega^{3}=0$, isto é, de $(3.25) v \in \mathcal{A}(\mathcal{I})$ se, e somente se, $v \in\left\{\omega^{1}, \omega^{2}, \omega_{3}^{1}, \omega_{3}^{2}\right\}^{\perp}$. Analogamente $v\lrcorner \Theta$ não envolve termos em $\omega^{3}$, portanto $\left.v\right\lrcorner \Theta \in \mathcal{I}$ se, e somente se, $v\lrcorner \Theta=0$, concluímos que $v \in\left\{\omega^{1}, \omega^{2}, \omega^{3}, \omega_{3}^{1}, \omega_{3}^{2}\right\}^{\perp}$, ou seja, $\mathcal{A}(\mathcal{I}) \subset\left\{\omega^{1}, \omega^{2}, \omega^{3}, \omega_{3}^{1}, \omega_{3}^{2}\right\}^{\perp}$. Reciprocamente, seja $v \in\left\{\omega^{1}, \omega^{2}, \omega^{3}, \omega_{3}^{1}, \omega_{3}^{2}\right\}^{\perp}$. Então $\left(v-\omega^{3}\right)(v)=\omega^{3}(v)=0$. Para 
$v\lrcorner d \omega^{3}$ tome $u \in T_{\rho(\widetilde{E})} \Sigma$ qualquer,

$$
\begin{aligned}
\left(v-d \omega^{3}\right)(u) & =d \omega^{3}(v, u) \\
& =\left(-\omega_{1}^{3} \wedge \omega^{1}-\omega_{2}^{3} \wedge \omega^{2}\right)(v, u) \\
& =-\underbrace{\omega_{1}^{3}(v)}_{0} \omega^{1}(u)+\omega_{1}^{3}(u) \underbrace{\omega^{1}(v)}_{0}-\underbrace{\omega_{2}^{3}(v)}_{0} \omega^{2}(u)+\omega_{2}^{3}(u) \underbrace{\omega^{2}(v)}_{0} \\
& =0,
\end{aligned}
$$

$\operatorname{logo} v\lrcorner d \omega^{3}=0$. Por fim $\left.v\right\lrcorner \Theta$, seja $u$ como anteriormente,

$$
\begin{aligned}
(v\lrcorner \Theta)(u) & =\left(A \omega_{1}^{3} \wedge \omega_{2}^{3}+B\left(\omega_{1}^{3} \wedge \omega^{2}-\omega_{2}^{3} \wedge \omega^{1}\right)+C \omega^{1} \wedge \omega^{2}\right)(v, u) \\
& =A \underbrace{\omega_{1}^{3}(v)}_{0} \omega_{2}^{3}(u)-A \omega_{1}^{3}(u) \underbrace{\omega_{2}^{3}(v)}_{0}+B \underbrace{\omega_{1}^{3}(v)}_{0} \omega^{2}(u)-B \omega_{1}^{3}(u) \underbrace{\omega^{2}(v)}_{0} \\
& -B \underbrace{\omega_{2}^{3}(v)}_{0} \omega^{1}(u)+B \omega_{2}^{3}(u) \underbrace{\omega^{1}(v)}_{0}+C \underbrace{\omega^{1}(v)}_{0} \omega^{2}(u)-C \omega^{1}(u) \underbrace{\omega^{2}(v)}_{0} \\
& =0,
\end{aligned}
$$

segue $v\lrcorner \Theta=0$, portanto $\left\{\omega^{1}, \omega^{2}, \omega^{3}, \omega_{3}^{1}, \omega_{3}^{2}\right\}^{\perp} \subset \mathcal{A}(\mathcal{I})$.

Podemos passar ao quociente pelas características de Cauchy, e assim omitir $\omega_{2}^{1}$, e do Exemplo A.2.11 temos que o espaço quociente tem dimensão 5. No quociente $\widetilde{E}$ fica determinado apenas por $h_{i j}$ e $\widetilde{E}$ é plano integral se $\left.\omega^{3}\right|_{\widetilde{E}}=0,\left.d \omega^{3}\right|_{\widetilde{E}}=0$ e $\left.\Theta\right|_{\widetilde{E}}=0$, a primeira restrição impõe para $v \in \widetilde{E}$ que $a(\widetilde{E})=b(\widetilde{E})=0$, a segunda é trivial pois

$$
\begin{aligned}
\left.d \omega^{3}\right|_{\widetilde{E}} & =-\left.\left.\omega_{1}^{3}\right|_{\widetilde{E}} \wedge \omega^{1}\right|_{\widetilde{E}}-\left.\left.\omega_{2}^{3}\right|_{\widetilde{E}} \wedge \omega^{2}\right|_{\widetilde{E}} \\
& =-\left.\left(\left.h_{11} \omega^{1}\right|_{\widetilde{E}}+\left.h_{12} \omega^{2}\right|_{\widetilde{E}}\right) \wedge \omega^{1}\right|_{\widetilde{E}}-\left.\left(\left.h_{21} \omega^{1}\right|_{\widetilde{E}}+\left.h_{22} \omega^{2}\right|_{\widetilde{E}}\right) \wedge \omega^{2}\right|_{\widetilde{E}} \\
& =\left.\left.\left(h_{12}-h_{21}\right) \omega^{1}\right|_{\widetilde{E}} \wedge \omega^{2}\right|_{\widetilde{E}} \\
& =0 .
\end{aligned}
$$

Resta calculara terceira.

$$
\begin{aligned}
0 & =\left.\Theta\right|_{\widetilde{E}} \\
& =A\left(h_{11} \omega^{1}+h_{12} \omega^{2}\right) \wedge\left(h_{21} \omega^{1}+h_{22} \omega^{2}\right)+B\left(\left(h_{11} \omega^{1}+h_{12} \omega^{2}\right) \wedge \omega^{2}\right. \\
& \left.-\left(h_{21} \omega^{1}+h_{22} \omega^{2}\right) \wedge \omega^{1}\right)+C \omega^{1} \wedge \omega^{2} \\
& \left.=A\left(h_{11} h_{22}-\left(h_{12}\right)^{2}\right)+B\left(h_{11}+h_{22}\right)+C\right) \omega^{1} \wedge \omega^{2} \\
& =\left(A \operatorname{det}\left(h_{i j}\right)+B\left(\operatorname{tr}\left(h_{i j}\right)\right)+C\right) \omega^{1} \wedge \omega^{2} .
\end{aligned}
$$


Como $\omega^{1} \wedge \omega^{2}=\Omega \neq 0$, temos

$$
A \operatorname{det}\left(h_{i j}\right)+B \operatorname{tr}\left(h_{i j}\right)+C=0 .
$$

Seja $V$ aberto de $\mathbb{R}_{i j} \cong \mathbb{R}^{3}$ formado pelas matrizes simétricas $h=\left(h_{i j}\right)$ e defina $F$ : $V \rightarrow \mathbb{R}, F\left(h_{i j}\right)=A \operatorname{det}\left(h_{i j}\right)+B \operatorname{tr}\left(h_{i j}\right)+C$. Então cada fibra de $V_{2}(\mathcal{I})$ é dada por $F^{-1}(\{0\})$ e $V_{2}(\mathcal{I})_{\rho(E)}$ é variedade diferenciável em torno de $E$ quando $d F_{h} \neq 0$ para cada $h \in F^{-1}(\{0\})$. Diferenciando $F$ obtemos

$$
d F_{h}=\left(\begin{array}{lll}
A h_{22}+B & -2 A h_{12} & A h_{11}+B
\end{array}\right)
$$

portanto $d F_{h}=0$ se, e somente se,

$$
\left\{\begin{array}{l}
A h_{22}+B=0 \\
-2 A h_{12}=0 \\
A h_{11}+B=0
\end{array}\right.
$$

isto é, exatamente nas matrizes $h=\left(\begin{array}{cc}\frac{-B}{A} & 0 \\ 0 & \frac{-B}{A}\end{array}\right)$. Em outras palavra, $\mathcal{V}_{2}(\mathcal{I})_{\rho(E)}$ é variedade diferenciável em torno de $E$ quando $A h+I B \neq 0$, para cada $h \in F^{-1}(\{0\})$. Observe que as fibras de $\mathcal{V}_{2}(\mathcal{I})$ têm dimensão 2 e os pontos em que $F$ tem diferencial nula só ocorrem se $F\left(-\frac{B}{A} I\right)=0$, isto é, $A C-B^{2}=0$, que por hipótese não acontece.

Podemos iniciar o Teste de Cartan. Seja $e \in E$ não nulo, denote $\left.x^{i}:=e\right\lrcorner \omega^{i}, i=1,2$. Vamos calcular os caracteres de

$$
\{0\}=E_{0} \subset E_{1} \subset E_{2}=E,
$$

em que $E_{1}=\operatorname{span}\{e\}$. Por definição $c_{0}=\operatorname{codim}(H(\{0\}))$ e $H(\{0\})$ é o espaço que anula as 1-forma de $\mathcal{I}$, isto é, $H(\{0\})=\operatorname{ker}\left(\omega^{3}\right), \operatorname{logo} s_{0}=c_{0}=\operatorname{codim}(H(\{0\}))=1$. Pelo item 5 da Proposição 3.3.6 temos que $v \in H\left(E_{1}\right)$ se, e somente se,

$$
\left\{\begin{array}{l}
d \omega^{3}(v, e)=0 \\
\Theta(v, e)=0 \\
\omega^{3}(v)=0
\end{array}\right.
$$

Estamos no quociente pelas características, então $\operatorname{dim}\left(T_{p} \Sigma\right)=5$. Como $\omega^{3}(v)=0, H\left(E_{1}\right)$ tem dimensão no máximo 4. Vamos analisar o que as duas primeiras equações de (3.27) 
impõem a $H\left(E_{1}\right) \cdot(3.27)$

$$
\left\{\begin{array}{l}
x^{1} \omega_{1}^{3}(v)+x^{2} \omega_{2}^{3}(v)=\omega_{1}^{3}(e) \omega^{1}(v)+\omega_{2}^{3}(e) \omega^{2}(v) \\
A \omega_{2}^{3}(e) \omega_{1}^{3}(v)-A \omega_{1}^{3}(e) \omega_{2}^{3}(v)+B x^{2} \omega_{1}^{3}(v)-B x^{1} \omega_{2}^{3}(v)= \\
B \omega_{1}^{3}(e) \omega^{2}(v)-B \omega_{2}^{3}(e) \omega^{1}(v)-C x^{2} \omega^{1}(v)+C x^{1} \omega^{2}(v)
\end{array}\right.
$$

colocando em matrizes,

$$
\underbrace{\left(\begin{array}{cc}
x^{1} & x^{2} \\
A \omega_{2}^{3}(e)+B x^{2} & -A \omega_{1}^{3}(e)-B x^{1}
\end{array}\right)}_{M}\left(\begin{array}{l}
\omega_{1}^{3}(v) \\
\omega_{2}^{3}(v)
\end{array}\right)=\left(\begin{array}{c}
\omega_{1}^{3}(e) \omega^{1}(v)+\omega_{2}^{3}(e) \omega^{2}(v) \\
\alpha \omega^{1}(v)+\beta \omega^{2}(v)
\end{array}\right)
$$

em que $\alpha=-\left(B \omega_{2}^{3}(e)+C x^{2}\right)$ e $\beta=B \omega_{1}^{3}(e)+C x^{1}$. Se $\operatorname{det}(M) \neq 0$, então $\omega_{1}^{3}(v)$ e $\omega_{2}^{3}(v)$ ficam em função de $\omega^{1}(v)$ e $\omega^{2}(v), \operatorname{logo} \operatorname{dim}\left(H\left(E_{1}\right)\right)=2$ e $c_{1}=\operatorname{codim}\left(H\left(E_{1}\right)\right)=3$, portanto $s_{1}=c_{1}-c_{0}=3-1=2$.

Calculando $\operatorname{det}(M)$ :

$$
\begin{aligned}
\operatorname{det}(M) & =x^{1}\left(-A \omega_{1}^{3}(e)-B x^{1}\right)-x^{2}\left(A \omega_{2}^{3}(e)+B x^{2}\right) \\
& =-A x^{1} \omega_{1}^{3}(e)-A x^{2} \omega_{2}^{3}(e)-B\left(\left(x^{1}\right)^{2}+\left(x^{2}\right)^{2}\right) \\
& =-A x^{1}\left(h_{11} x^{1}+h_{12} x^{2}\right)-A x^{2}\left(h_{21} x^{1}+h_{22} x^{2}\right)-B\left(\left(x^{1}\right)^{2}+\left(x^{2}\right)^{2}\right) \\
& =-A h_{11}\left(x^{1}\right)^{2}-2 A h_{12} x^{1} x^{2}-A h_{22}\left(x^{2}\right)^{2}-B\left(\left(x^{1}\right)^{2}+\left(x^{2}\right)^{2}\right) .
\end{aligned}
$$

Como $x^{i}$ depende de $e$, basta tomar $e$ de forma que $\operatorname{det}(M) \neq 0$.

Do item 2 da Proposição 3.3.6, $E$ é plano integral, então $E \subset H\left(E_{1}\right)$, como ambos têm dimensão 2 , vale a igualdade. Resta apenas $s_{2}$, mas do fato que $c_{2}=\operatorname{codim}\left(E_{2}=E\right)=3$, temos $s_{2}=c_{2}-c_{1}=3-3=0$, então $\operatorname{dim}\left(\mathcal{V}_{2}(\mathcal{I})_{\rho(E)}\right)=2=s_{1}+2 s_{2}=2+2 \times 0$, portanto involutivo. Da $3^{\text {a }}$ versão do Teorema de Cartan-Kähler, as variedades integrais dependem de duas funções de uma variável. 


\section{Característica de Cauchy}

\section{A.1 Simetrias}

Definição A.1.1. Seja vum $\in \Gamma(T \Sigma)$ campo. O fluxo de $v$ é a aplicação $\varphi: U \subset \Sigma \times \mathbb{R} \rightarrow$ $\Sigma, U$ aberto, tal que

$$
\frac{\partial}{\partial t} \varphi(q, t)=v_{\varphi(q, t)}
$$

Definimos a derivada de Lie de $\alpha \in \Omega^{1}(\Sigma)$ com respeito a $v$, denotamos $\mathcal{L}_{v} \alpha \in \Omega^{1}(\Sigma)$, por

$$
\mathcal{L}_{v} \alpha:=\lim _{t \rightarrow 0} \frac{\varphi_{t}^{*} \alpha-\alpha}{t} .
$$

Analogamente para campos $v, w \in \Gamma(T \Sigma)$

$$
\mathcal{L}_{v} w=\lim _{t \rightarrow 0} \frac{\left(\varphi_{-t}\right)_{*} Y-Y}{t}
$$

Proposição A.1.2. No contexto da definição anterior, valem as seguintes propriedades:

1. $\mathcal{L}_{v} w=[v, w]$

2. $\mathcal{L}_{v}(\alpha \wedge \beta)=\mathcal{L}_{v} \alpha \wedge \beta+\alpha \wedge \mathcal{L}_{v} \beta$

3. $\left.\left.\mathcal{L}_{v} \alpha=v\right\lrcorner d \alpha+d(v\lrcorner \alpha\right)$

4. $d\left(\mathcal{L}_{v} \alpha\right)=\mathcal{L}_{v} d \alpha$

5. $\mathcal{L}_{[v, w]} \alpha=\mathcal{L}_{v} \mathcal{L}_{w} \alpha-\mathcal{L}_{w} \mathcal{L}_{v} \alpha$

6. $\left.\left.[v, w]\lrcorner \alpha=\mathcal{L}_{v}(w\lrcorner \alpha\right)-w\right\lrcorner\left(\mathcal{L}_{v} \alpha\right)$.

Demonstração. [Lee, 2012, p. 230, 372-373].

Definição A.1.3. Seja $\mathcal{I}$ um sistema diferencial exterior em $\Sigma$. Um campo $v \in \Gamma(T \Sigma)$ é dito uma simetria de $\mathcal{I}$ se $\mathcal{L}_{v} \psi \in \mathcal{I}, \forall \psi \in \mathcal{I}$. 
Proposição A.1.4. Sejam $v, w$ simetrias de $\mathcal{I}$, então $[v, w]$ também é simetria, isto é, as simetrias de $\mathcal{I}$ formam um álgebra de Lie.

Demonstração. Para cada $\alpha \in \mathcal{I}, \mathcal{L}_{v} \alpha \in \mathcal{I}$, já que $v$ é simetria, e usando a simetria de $w, \mathcal{L}_{w} \mathcal{L}_{v} \alpha \in I$, análogo para $\mathcal{L}_{v} \mathcal{L}_{w} \alpha$, temos pelo item 5 da Proposição A.1.2 que $\mathcal{L}_{[v, w]} \alpha \in \mathcal{I}$.

Observação A.1.5. Para verificar se $v$ é simetria basta verificar em um conjunto gerador de $\mathcal{I}$. De fato, suponha $\mathcal{I}=\left\{\theta_{\alpha}\right\}_{\alpha \in A}$, seja $\omega \in \mathcal{I}$, podemos escrever $\omega=\omega_{\alpha} \wedge \theta^{\alpha}$, em que $\alpha \in A^{\prime}$ finito e $\omega_{\alpha} \in \Omega^{*}(\Sigma)$, se $v$ é simetria para cada $\theta^{\alpha}$, então

$$
\mathcal{L}_{v}\left(\omega_{\alpha} \wedge \theta^{\alpha}\right)=\mathcal{L}_{v} \omega^{\alpha} \wedge \theta^{\alpha}+\omega_{\alpha} \wedge \mathcal{L}_{v} \theta^{\alpha}
$$

o primeiro termo está em $\mathcal{I}$ pois é produto com $\theta^{\alpha}$, o segundo está em $\mathcal{I}$ pois é produto $\operatorname{com} \mathcal{L}_{v} \theta^{\alpha}$ que está em $\mathcal{I}$, uma vez que $v$ é simetria de $\theta^{\alpha}$, portanto $v$ simetria de $\mathcal{I}$.

Exemplo A.1.6. Sejam $\Sigma=\mathbb{R}^{3}, \theta=d y-z d x$ e $\mathcal{I}=\{\theta\}_{\text {diff }}$. O campo $v=f \frac{\partial}{\partial x}+$ $g \frac{\partial}{\partial y}+\left(g_{x}+z g_{y}-z f_{x}-z^{2} f_{y}\right) \frac{\partial}{\partial z}$, em que $f, g$ são funções em $(x, y)$ apenas, é simetria de $\mathcal{I}$. É suficiente verificar apenas $\mathcal{L}_{v} \theta$, mas $\mathcal{L}_{v} \theta=\mathcal{L}_{v}(d y-z d x)$, pelo item 3 da Proposição A.1.2 $\left.\left.\mathcal{L}_{v}(d y-z d x)=v\right\lrcorner(-d z \wedge d x)+d(v\lrcorner(d y-z d x)\right)$. Vamos calcular cada termo,

$$
\begin{aligned}
v\lrcorner(-d z \wedge d x)(w) & =-d z \wedge d x(v, w) \\
& =-d z(v) d x(w)+d z(w) d x(v) \\
& =-\left(g_{x}+z g_{y}-z f_{x}-z^{2} f_{y}\right) d x(w)+f d z(w) \\
& =\left(-\left(g_{x}+z g_{y}-z f_{x}-z^{2} f_{y}\right) d x+f d z\right)(w)
\end{aligned}
$$

$\operatorname{assim} v\lrcorner(-d z \wedge d x)=-\left(g_{x}+z g_{y}-z f_{x}-z^{2} f_{y}\right) d x+f d z$.

$$
\begin{aligned}
d(v-(d y-z d x)) & =d(g-z f) \\
& =g_{x} d x+g_{y} d y-f d z-z f_{x} d x-z f_{y} d y
\end{aligned}
$$

somando,

$$
\begin{aligned}
\mathcal{L}_{v} \theta & =-z g_{y} d x+z^{2} f_{y} d x+g_{y} d y-z f_{y} d y \\
& =-\left(g_{y}+z f_{y}\right) z d x+\left(g_{y}-z f_{y}\right) d y \\
& =\left(g_{y}+z f_{y}\right)(d y-z d x) \\
& =\left(g_{y}+z f_{y}\right) \theta
\end{aligned}
$$

concluímos que $\mathcal{L}_{v} \theta \in \mathcal{I}$ e $v$ simetria. 
Proposição A.1.7. Sejam $\mathcal{I}$ sistema diferencial exterior em $\Sigma, N_{0}$ variedade integral $e$ $v$ simetria de $\mathcal{I}$. Considere $\varphi: U \subset \Sigma \times \mathbb{R} \rightarrow \Sigma$ fluxo de $v$ e defina $N_{t}=\varphi_{t}\left(N_{0}\right)$, se $N_{t}$ for diferenciável, então é variedade integral de $\mathcal{I}$.

Demonstração. Fixe $t \in \mathbb{R}$ e denote $i: N_{0} \rightarrow \Sigma$ inclusão, defina $f: N_{t} \rightarrow \Sigma$ por $f=i \circ \phi_{t}^{-1}$, seja $\alpha \in \mathcal{I}$, observe que $f^{*}(\alpha)=\left(i \circ \phi_{t}^{-1}\right)^{*}(\alpha)=\left(\phi_{t}^{-1}\right)^{*}\left(i^{*}(\alpha)\right)$, como $i^{*}(\alpha)=0$, segue $f^{*}(\alpha)=0$ e $N_{t}$ variedade integral de $\mathcal{I}$.

\section{A.2 Campos característicos}

Definição A.2.1. Seja $\mathcal{I}$ sistema diferencial exterior em $\Sigma$, um campo $v \in \Gamma(T \Sigma)$ é dito campo característico de Cauchy se $v\lrcorner \alpha \in \mathcal{I}, \forall \alpha \in \mathcal{I}$. As curvas integrais de $v$ são ditas curvas características de Cauchy.

Proposição A.2.2. Sejam $\mathcal{I}$ sistema diferencial exterior em $\Sigma$ e v campo característico de Cauchy, então v é simetria de $\mathcal{I}$. Se w é outro campo característico, $[v, w]$ é também campo característico de Cauchy.

Demonstração. Primeiro a simetria, temos $\left.\left.\mathcal{L}_{v} \alpha=v\right\lrcorner d \alpha+d(v\lrcorner \alpha\right)$, do fato que $\alpha \in \mathcal{I}$ segue $d \alpha \in I$, e $v$ ser campo característico nos dá $v\lrcorner d \alpha \in \mathcal{I}$, para o segundo termo o raciocínio é análogo, $v\lrcorner \alpha \in \mathcal{I}$ por $v$ ser característico e sua derivada está em $\mathcal{I}$, uma vez que $\mathcal{I}$ é fechado. A segunda afirmação: como $w$ característico, segue $w\lrcorner \alpha \in \mathcal{I}$, $v$ em particular é simetria, portanto $\left.\mathcal{L}_{v}(w\lrcorner \alpha\right) \in \mathcal{I}$, usando argumento análogo para $w\lrcorner\left(\mathcal{L}_{v} \alpha\right) \in \mathcal{I}$, do item 6 da Proposição A.1.2 obtemos o resultado.

Observação A.2.3. Suponha que $\mathcal{I}$ é gerado algebricamente por $\left\{\theta^{a}\right\}$, vale em geral

$$
\left.\left.v\lrcorner\left(\alpha \wedge \theta^{a}\right)=(v\lrcorner \alpha\right) \wedge \theta^{a}+(-1)^{\operatorname{deg}(\alpha)} \alpha \wedge(v\lrcorner \theta^{a}\right),
$$

se $v\lrcorner \theta^{a} \in \mathcal{I}$, então (A.1) garante que $v$ é característico.

Exemplo A.2.4. Considere a equação $u_{t}=u_{x}$. Em $J^{1}\left(\mathbb{R}^{2}, \mathbb{R}\right)$, com coordenadas $x^{1}, x^{2}$, $p_{1}, p_{2}$ e $u$, a equação anterior é equivalente ao sistema de contato canônico na subvariedade $\Sigma \subset J^{1}$ definida por $p_{1}=p_{2}$, denote $x=x^{1}$ e $t=x^{2}, p=p_{1}$ e mantenha $u$, coordenadas em $\Sigma$, o sistema de contato canônico é gerado por $\theta=d u-p d x-p d t=d u-p(d x+d t)$ e denote $\mathcal{I}=\{\theta\}$, claro que $d \theta=-d p \wedge(d x+d y)$. Suponha

$$
v=a \frac{\partial}{\partial x}+b \frac{\partial}{\partial t}+c \frac{\partial}{\partial p}+d \frac{\partial}{\partial u}
$$


característico em $\Sigma, v\lrcorner \theta \in \mathcal{I}$, impõe $v\lrcorner \theta=0$, assim

$$
\begin{aligned}
0 & =v\lrcorner \theta \\
& =(d u-p(d x+d t))\left(a \frac{\partial}{\partial x}+b \frac{\partial}{\partial t}+c \frac{\partial}{\partial p}+d \frac{\partial}{\partial u}\right) \\
& =d-p(a+b),
\end{aligned}
$$

por outro lado, se $v\lrcorner d \theta \in \mathcal{I}$, impõe $v\lrcorner d \theta \equiv 0$, em $\Sigma$, então para todo $w \in T_{(x, t, p, u)} \Sigma$ vale

$$
\begin{aligned}
(v-(-d p \wedge(d x+d t)))(w) & =(-d p \wedge(d x+d t))(v, w) \\
& =(-c(d x+d t)+(a+b) d p)(w)
\end{aligned}
$$

isto é, $v\lrcorner(-d p \wedge(d x+d t))=c(d x+d t)-(a+b) d p$ como $d x+d t, d p$ e $\theta$ são linearmente independentes, segue $c=0$ e $a+b=0, \log \alpha=p(a+b)=0$, assim escrevemos $v=a \frac{\partial}{\partial x}-a \frac{\partial}{\partial t}=a\left(\frac{\partial}{\partial x}-\frac{\partial}{\partial t}\right)$. Observe que $\gamma(s)=\left(a s, c_{1}-a s, c_{2}, c_{2}, c_{3}\right)$, em que $c_{i}$ são constantes, é curva integral de $v$, já que $\gamma^{\prime}(s)=a \frac{\partial}{\partial x}-a \frac{\partial}{\partial t}=v$, então podemos construir superfícies integrais de $\mathcal{I}$ transladando curvas integrais de $\mathcal{I}$ ao longo de $\gamma$.

Proposição A.2.5. Sejam $\mathcal{I}$ sistema diferencial exterior em $\Sigma, N_{0}$ variedade integral de $\mathcal{I}$ e $v$ campo característico de $\mathcal{I}$ transversal a $N_{0}$. Considere $N_{t}$ como na Proposição A.1.7, se $S=\bigcup N_{t}$ for diferenciável, então $S$ é variedade integral de $\mathcal{I}$.

Demonstração. Suponha $S$ diferenciável, seja $p \in S$, então existe $t \in \mathbb{R}$ tal que $p \in N_{t}$, tome $\left\{w_{1}, \cdots, w_{n}\right\}$ base de $T_{p} N_{t}$, como $v$ é transversal, $\left\{v, w_{1}, \cdots, w_{n}\right\}$ é base de $T_{p} S$. Seja $\alpha \in \mathcal{I}$, com $\operatorname{deg}(\alpha)=k<n+1$, como $N_{t}$ é variedade integral de $\mathcal{I}$, segue que $\alpha\left(w_{i^{1}}, \cdots, w_{i^{k}}\right)=0$, por outro lado $\left.\alpha\left(v, w_{i^{1}}, \cdots, w_{i^{k-1}}\right)=(v\lrcorner \alpha\right)\left(w_{i^{1}}, \cdots, w_{i^{k-1}}\right)=0$, uma vez que $v$ é característico, isto é, $v\lrcorner \alpha \in \mathcal{I}$. Portanto $\alpha=0$ em $S$, ou seja, $S$ é variedade integral de $\mathcal{I}$.

Exemplo A.2.6. Considere a equação $u_{t}=u u_{x}$ para funções do $\mathbb{R}^{2}$ em $\mathbb{R}$. Seja $J^{1}\left(\mathbb{R}^{2}, \mathbb{R}\right)$ com coordenadas $(x, t, p, q, u)$, defina $\Sigma \subset J^{1}\left(\mathbb{R}^{2}, \mathbb{R}\right)$ por $q=u p$, então a forma de contato canônica em $\Sigma$ é $\theta=d u-p d x-u p d t$. Denote $\mathcal{I}:=\{\theta\}_{\text {diff }}$,

$$
\begin{aligned}
d \theta & \equiv-d p \wedge d x-u d p \wedge d t-p d u \wedge d t \bmod \mathcal{I} \\
& \equiv-d p \wedge d x-u d p \wedge d t-p(\theta+p d x+u p d t) \wedge d t \bmod \mathcal{I} \\
& \equiv-d p \wedge d x-u d p \wedge d t-p^{2} d x \wedge d t-u p^{2} d t \wedge d t \bmod \mathcal{I} \\
& \equiv-\left(d p-p^{2} d t\right) \wedge(d x+u d t) \bmod \mathcal{I}
\end{aligned}
$$


Se $v=a \frac{\partial}{\partial x}+b \frac{\partial}{\partial t}+c \frac{\partial}{\partial p}+d \frac{\partial}{\partial u}$, então

$$
\left\{\begin{array}{l}
v\lrcorner \theta \in \mathcal{I} \Longrightarrow v\lrcorner \theta=0 \\
v\lrcorner d \theta \in \mathcal{I} \Longrightarrow v\lrcorner d \theta \equiv 0 \bmod \mathcal{I}
\end{array}\right.
$$

isto é,

$$
\left\{\begin{array}{l}
d-p(a+u b)=0 \\
c-p^{2} b=0 \\
a+u b=0
\end{array}\right.
$$

como temos grau de liberdade, tome $b=1, \operatorname{logo} a=-u, c=p^{2}$ e $d=0$, isto é $v=-u \frac{\partial}{\partial x}+\frac{\partial}{\partial t}+p^{2} \frac{\partial}{\partial p}$ é vetor característico. Uma curva integral de $v$ é dada por

$$
\left\{\begin{array}{l}
\frac{d x}{d t}=-u \\
\frac{d u}{d t}=0 \\
\frac{d p}{d t}=p^{2}
\end{array}\right.
$$

cuja solução é $x(t)=x_{0}-u t, u(t)=u_{0}$ e $p(t)=\frac{1}{p_{0}^{-1}-t}$. Observe que a imagem de $\gamma(s)=$ $\left(s, 0, f^{\prime}(s), f(s) f^{\prime}(s), f(s)\right)$, para $f$ função diferenciável, é variedade integral de $\mathcal{I}$, já que $\gamma^{*}(\theta)=(d u-p d x-u p d t)\left(\frac{\partial}{\partial x}+f^{\prime \prime}(s) \frac{\partial}{\partial p}+f^{\prime}(s) \frac{\partial}{\partial u}\right)=f^{\prime}(s)-f^{\prime}(s) \times 1-f(s) f^{\prime}(s) \times 0=0$. Pelo teorema anterior, uma superfície integral de $\mathcal{I}$ pode ser obtida transladando $\gamma$ via curva integral de $v$, isto é, $x_{0}=s, u_{0}=f(s)$ e $p_{0}=f^{\prime}(s)$ nos da $u=f(s)=f(x+u t)$, note que $p_{0}(s) \neq \frac{1}{t}$, logo $t p^{\prime}(s)-1=t f^{\prime}(x+u t)-1 \neq 0$, o que garante $u$, dada implicitamente, solução de $u_{t}=u u_{x}$ e seu gráfico variedade integral de $\mathcal{I}$.

Definição A.2.7. Dizemos que um subfibrado $J \subset T^{*} \Sigma$ é Frobenius se o ideal algébrico gerado pelas seções de $J$ é também um ideal diferencial.

Definição A.2.8. Sejam $\mathcal{I}$ sistema diferencial exterior em $\Sigma$ e $x \in \Sigma$, defina

$$
\left.\mathcal{A}(\mathcal{I})_{x}:=\left\{v \in T_{x} \Sigma: v\right\lrcorner \mathcal{I} \subset \mathcal{I}\right\}
$$

$\mathcal{A}(\mathcal{I})_{x}$ é subespaço linear. Utilizando a Proposição A.2.2, $\mathcal{A}(\mathcal{I})$ é fechado pelo colchete de Lie. O dual de $\mathcal{A}(\mathcal{I})_{x}$

$$
\left.\mathcal{C}(\mathcal{I})_{x}:=\left\{\theta \in T_{x}^{*} \Sigma: v\right\lrcorner \theta=0, \forall v \in \mathcal{A}(\mathcal{I})_{x}\right\}
$$


é dito sistema de Cartan. Sejam $v, w \in \mathcal{A}(\mathcal{I})$ e $\theta$ seção de $\mathcal{C}(\mathcal{I})$, então

$$
\begin{aligned}
d \theta(v, w) & =v(w\lrcorner \theta)-w(v\lrcorner \theta)-[v, w]\lrcorner \theta \\
& =0,
\end{aligned}
$$

$\operatorname{assim} \mathcal{C}(\mathcal{I})$ é Frobenius. Aplicando o Teorema de Frobenius, existe sistema de coordenadas $\left(x^{i}\right)$ tal que $\mathcal{C}(\mathcal{I})=\left\{d x^{1}, \cdots, d x^{s}\right\}$, em que $s \leq n$.

Proposição A.2.9. O sistema de Cartan acima é o menor sistema Frobenius tal que $\Lambda \mathcal{C}(\mathcal{I})$ contém os geradores algébricos de $\mathcal{I}$.

Demonstração. Seja $J$ Frobenius tal que as seguintes formas $\psi^{1}, \cdots, \psi^{m} \in \Lambda J$ geram $J$ algebricamente. Dada $\Phi \in \mathcal{I}$, então $\Phi=\beta_{k} \wedge \psi^{k}$, se $v$ é tal que $v-\alpha=0, \forall \alpha \in J$, então $\left.v\lrcorner \Phi=(v\lrcorner \beta_{k}\right) \wedge \psi^{k}+(-1)^{\operatorname{deg}\left(\beta_{k}\right)} \beta_{k} \wedge \underbrace{\left.(v\lrcorner \psi^{k}\right)}_{0}=(v\lrcorner \beta_{k}) \wedge \psi^{k} \in \mathcal{I}$, como $v \mathrm{e}$ $\Phi$ são arbitrárias, segue $\mathcal{C}(\mathcal{I}) \subset J$. Não sabemos se os geradores de $\mathcal{I}$ estão em $\Lambda \mathcal{C}(\mathcal{I})$, apenas que $\mathcal{C}(\mathcal{I})$ está contido no menor $J$, suponha que a codimensão de $\mathcal{C}(\mathcal{I})$ em $J$ seja $l>0$ e $\pi_{1}, \cdots, \pi_{l}$ são formas linearmente independentes tais que $\pi_{i} \notin \mathcal{C}(\mathcal{I})$, Particione os geradores de $\mathcal{I}$ em $\phi_{1}, \cdots, \phi_{m} \in \Lambda \mathcal{C}(\mathcal{I})$ e $\psi_{1}, \cdots, \psi_{n}$ geradores que envolvam $\pi_{i}$ nos seus termos, podemos assumir também que os graus são crescentes. Seja $v \in \mathcal{A}(\mathcal{I})$ e $\psi_{1}$ com menor grau, $v\lrcorner \psi_{1}$, devido ao grau, $\left.v\right\lrcorner \psi_{1}$ pode ser expresso em termos de $\phi$ 's, logo podemos omitir os termos de $\psi_{1}$ que envolvem $\pi_{i}$, uma vez que esse termos são expressos em ternos de $\phi$ 's, recursivamente temos que todos os geradores de $\mathcal{I}$ estão em $\Lambda \mathcal{C}(\mathcal{I})$.

Definição A.2.10. Seja $\mathcal{I}$ sistema diferencial exterior em $\Sigma$, suponha $\mathcal{C}(\mathcal{I})$ com posto constante $k$, então as variedades integrais maximais de $\mathcal{C}(\mathcal{I})$ forma uma folheação de $\Sigma$ por subvariedades de codimensão $k$, se o espaço das folhas é uma variedade diferenciável, digamos $Q$, dizemos que $Q$ é o quociente pelas características de Cauchy.

Exemplo A.2.11. Sejam $A S O(3), p \in A S O(3)$ e $\omega$ forma de Maurer-Cartan em $A S O(3)$. Considere $\mathcal{I}=\left\{\omega^{3}\right\}_{\text {diff }}$, as variedades integrais de $\mathcal{I}$ são exatamente os fibrados adaptados de $M^{2} \subset \mathbb{E}^{3}$. Denote $\left(\omega^{j}\right)^{*}$ e $\left(\omega_{j}^{i}\right)^{*}$ vetores duais a $\omega^{i}$ e $\omega_{j}^{i}$, então $v \in T_{p}(A S O(3))$ é escrito como $v=a_{j}\left(\omega^{j}\right)^{*}+a_{i}^{j}\left(\omega_{j}^{i}\right)^{*}, v \in \mathcal{A}(\mathcal{I})$ implica em

$$
\left\{\begin{array}{l}
v\lrcorner \omega^{3}=0 \\
v\lrcorner d \omega^{3} \in \mathcal{I}^{1},
\end{array}\right.
$$


a primeira igualdade implica em $a_{3}=0$. A segunda

$$
\begin{aligned}
v\lrcorner d \omega^{3} & =v\lrcorner\left(-\omega_{1}^{3} \wedge \omega^{1}-\omega_{2}^{3} \wedge \omega^{2}\right) \\
& =a_{1} \omega_{1}^{3}-a_{3}^{1} \omega^{1}+a_{2} \omega_{2}^{3}-a_{3}^{2} \omega^{2}
\end{aligned}
$$

como as 1-formas de $\mathcal{I}^{1}$ são múltiplos de $\omega^{3}$ segue que $a_{1}=a_{3}^{1}=a_{2}=a_{3}^{2}=0$, isto é $\mathcal{A}(\mathcal{I})$ é gerado por $v=\left(\omega_{2}^{1}\right)^{*}$.

Nesse caso $\mathcal{C}(\mathcal{I})$ é gerado por $\left\{\omega^{1}, \omega^{2}, \omega^{3}, \omega_{1}^{3}, \omega_{2}^{3}\right\}$, as curvas integrais de $\mathcal{C}(\mathcal{I})$ formam uma folheação de $A S O(3)$. O espaço quociente pelas características de Cauchy é homogêneo e de dimensão 5 , podemos identificar esse espaço com o $S \mathbb{E}^{3}$, fibrado unitário de $\mathbb{E}^{3}$.

O seguinte a seguir estabelece a relação do sistema diferencial $\mathcal{I}$ com o espaço quociente.

Teorema A.2.12. Seja $\mathcal{I}$ um sistema diferencial exterior em $\Sigma$, suponha que o quociente pelas características de Cauchy é uma variedade direnciável $Q$, então existe um sistema diferencial exterior $\tilde{\mathcal{I}}$ em $Q$ tal que $\mathcal{I}=\left\{\pi^{*} \tilde{\mathcal{I}}\right\}_{\text {alg }}$, em que $\pi: M \rightarrow Q$ projeção.

Demonstração. [Thomas A. Ivey, 2003, p. 221-222] 


\section{Referências Bibliográficas}

[Allendoerfer, 1940] Allendoerfer, C. B. (1940). The euler number of a riemann manifold. American Journal of Mathematics, 62(1):243-248.

[Carl B. Allendoerfer, 1943] Carl B. Allendoerfer, A. W. (1943). The gauss-bonnet theorem for riemannian polyhedra. Transactions of the American Mathematical Society, 53(1):101-129.

[Chern, 1944] Chern, S.-S. (1944). A simple intrinsic proof of the gauss-bonnet formula for closed riemannian manifolds. Annals of Mathematics, 45(4):747-752.

[Chern, 1945] Chern, S.-S. (1945). On the curvatura integra in a riemannian manifold. Annals of Mathematics, 46(4):674-684.

[Darboux, 1887] Darboux, G. (1887). Leçons sur la théorie générale des surfaces, volume Part 1. Paris.

[Fenchel, 1940] Fenchel, W. (1940). On total curvatures of riemannian manifolds: I. Journal of the London Mathematical Society, s1-15(1):15-22.

[Guillemin, 1974] Guillemin, P. (1974). Differential Topology. Prentice Hall.

[Kuranishi, 1957] Kuranishi, M. (1957). On e. cartan's prolongation theorem of exterior differential systems. American Journal of Mathematics, 79(1):1-47.

[Kuranishi, 1967] Kuranishi, M. (1967). Lectures on involutive systems of PDEs. Sociedade de Matemática de São Paulo.

[Lee, 1997] Lee, J. M. (1997). Riemannian Manifolds: An Introduction to Curvature. Graduate texts in mathematics. Springer, 1 edition.

[Lee, 2012] Lee, J. M. (2012). Introduction to Smooth Manifolds. Graduate Texts in Mathematics 218. Springer-Verlag New York, 2 edition. 
[Lipman Bers, 1971] Lipman Bers, Fritz John, M. S. (1971). Partial Differential Equations. Lectures in Applied Mathematics 3. The American Mathematical Society.

[M. A. Akivis, 1993] M. A. Akivis, B. A. R. (1993). Elie Cartan (1869-1951). Translations of Mathematical Monographs, v.123. American Mathematical Society.

[Morita, 2001] Morita, S. (2001). Geometry of differential forms. Translations of mathematical monographs, Iwanami series in modern mathematics 201. American Mathematical Society.

[Raoul Bott, 1995] Raoul Bott, L. T. (1995). Differential forms in algebraic topology. Graduate texts in mathematics 82. Springer.

[Robert L. Bryant, 1991] Robert L. Bryant, S.-S. Chern, R. B. G. H. L. G. P. A. G. a. (1991). Exterior Differential Systems. Mathematical Sciences Research Institute Publications 18. Springer-Verlag New York, 1 edition.

[Spivak, 1999a] Spivak, M. (1999a). A Comprehensive Introduction to Differential Geometry, volume 1. Publish or Perish, 3rd edition.

[Spivak, 1999b] Spivak, M. (1999b). A Comprehensive Introduction to Differential Geometry, volume 5. Publish or Perish, 3rd edition.

[Sternberg, 1964] Sternberg, S. (1964). Lectures on differential geometry. Prentice-Hall mathematics series. Prentice-Hall, 1 edition.

[Thomas A. Ivey, 2003] Thomas A. Ivey, J. M. L. (2003). Cartan for beginners: differential geometry via moving frames. Graduate Studies in Mathematics. American Mathematical Society,.

[Weiping Zhang, 2001] Weiping Zhang, Z. W.-P. (2001). Lectures on Chern-Weil Theory and Witten Deformations. Nankai tracts in mathematics 4. World Scientific, 1 edition.

[Élie Cartan, 1955a] Élie Cartan (1955a). La structure des groupes de transformations continus et la théorie du trièdre mobile. Bulletin des Sciences Mathématiques, 1-2:145178.

[Élie Cartan, 1955b] Élie Cartan (1955b). Sur les développables isotropes et la méthode du trièdre mobile. Société mathématique de France, 1-2:141-143. 
[Émile Cotton, 1905] Émile Cotton (1905). Généralisation de la théorie du trièdre mobile. Bulletin de la Société Mathématique de France, 33:42-64. 
